\title{
Charge Transport in Sequence-Defined Conjugated Oligomers
}

Hao $\mathrm{Yu}^{1 \dagger}$, Songsong $\mathrm{Li}^{2,4 \dagger}$, Kenneth E. Schwieter ${ }^{3}$, Yun Liu ${ }^{3,4}$, Boran Sun ${ }^{1}$, Jeffrey S. Moore ${ }^{2,3,4}$, and Charles M. Schroeder ${ }^{1,2,3,4^{*}}$

${ }^{1}$ Department of Chemical and Biomolecular Engineering, University of Illinois at UrbanaChampaign, Urbana, Illinois 61801, United States

${ }^{2}$ Department of Materials Science and Engineering, University of Illinois at Urbana-Champaign, Urbana, Illinois 61801, United States

${ }^{3}$ Department of Chemistry, University of Illinois at Urbana-Champaign, Urbana, Illinois 61801, United States

${ }^{4}$ Beckman Institute for Advanced Science and Technology, University of Illinois at UrbanaChampaign, Urbana, Illinois 61801, United States

${ }^{\dagger}$ Contributed equally to this work.

*To whom correspondence should be addressed.

Email: cms@,illinois.edu

\section{Contents:}

1. General Methods $\quad$ S2

2. Synthetic Details $\quad$ S4

3. ${ }^{1} \mathrm{H}$ and ${ }^{13} \mathrm{C}$ NMR Spectra $\quad$ S19

4. Single Crystal X-ray Crystallographic Analysis $\quad$ S56

5. Single Molecule Conductance Measurements $\quad$ S64

6. Density Functional Theory (DFT) Simulations $\quad$ S77

7. Conformation Analysis $\quad$ S79

8. UV-vis Spectra $\quad$ S84

9. References $\quad$ S85 


\section{General Methods}

\section{Materials}

All reagents were purchased from commercial suppliers and used as received unless otherwise stated. Dry dichloromethane (DCM), dimethylformamide (DMF) and tetrahydrofuran (THF) were obtained from a Solvent Delivery System (SDS) equipped with activated neutral alumina columns under argon. Column chromatography were performed on Biotage Isolera System using Silicycle Siliasep HP flash cartridges.

\section{Characterization}

NMR. ${ }^{1} \mathrm{H}$ and ${ }^{13} \mathrm{C}$ NMR spectra $(500 \mathrm{MHz}$ and $125 \mathrm{MHz}$ ) were recorded at room temperature (298 $\mathrm{K})$. Chemical shifts are reported in $\delta(\mathrm{ppm})$ referenced on residual solvent peaks. Coupling constants $(\mathrm{J})$ are expressed in Hertz $(\mathrm{Hz})$. Splitting patterns are designated as: s (singlet), $\mathrm{d}$ (doublet), $\mathrm{t}$ (triplet), $\mathrm{m}$ (multiplet).

\section{Mass-Spectrometry}

High resolution ESI mass spectra were recorded on a Waters Q-TOF Ultima ESI spectrometer.

\section{X-ray Crystallographic Analysis}

Single crystals suitable for X-ray crystallography were grown by slow evaporation of a solution mixture of $\mathrm{CHCl}_{3} / \mathrm{n}$-Hexane or EtOAc/n-Hexane. Bruker X8ApexII (APEX) was used for the data collection.

\section{Single Molecule Conductance Measurement}

All single molecule conductance measurements were performed in a home-built scanning tunneling microscope setup, using a method as previously described. ${ }^{1}$ Gold tips were prepared using $0.25 \mathrm{~mm}$ Au wire (99.998\%, Alfa Aesar). Gold substrates were prepared by evaporating 120 $\mathrm{nm}$ of gold onto polished AFM metal specimen discs (Ted Pella). Conductance measurements were carried out in $1 \mathrm{mM}$ molecule solution in 1,2,4-trichlorobenzene. The break junction experiments were conducted at a bias from $250 \mathrm{mV}$ to $750 \mathrm{mV}$. All histograms (over 5,000 traces for each molecule) are constructed without any data selection.

\section{Conformation Analysis by Density Function Theory(DFT) Simulation}

All computations have been carried out using the B3LYP functional with Gaussian '09. ${ }^{2}$ All oligomers were studied in their most stable conformers. The solubilizing side chains were shortened to two carbon atoms in their longest backbone. Solvation effect of 1,2,4trichlorobenzene $\left(\varepsilon_{\mathrm{r}}=2.24\right)$ was implicitly taken into account for all calculations using CPCM model. ${ }^{3}$ Geometry optimizations were performed using the B3LYP functional with a 6-31G(d,p) basis set, tight convergence criteria, and ultrafine integration grids. Vibrational analyses were conducted with the same level of theory to verify the stationary points to be minimum energy structures. Single-point energies, HOMO-LUMO orbitals and their energies were calculated at a higher B3LYP/6-311++G(d,p) level of theory.

\section{DFT Simulation on Single Molecule Conductance}

Electron transport calculations were calculated using nonequilibrium Green's function/density functional theory method via the Atomistix Toolkit package. ${ }^{4-6}$ We optimize all molecules using Spartan with a 6-31 $\mathrm{G}^{* *}$ basis. Then we place the optimized molecules in built junctions and relax all atoms to $0.05 \mathrm{ev} / \AA$ using DFT with local spin density approximation, a double- $\zeta$ polarized basis 
set for the molecules except for gold atoms with a single- $\zeta$ basis set, and k-point samplings of $3 \mathrm{x}$ $3 \times 50$ (with 50 being in the direction along the transport). Finally, we calculated the transmission spectra through the junction.

\section{UV-Vis}

UV-Vis absorption spectra were recorded in HPLC Grade Chloroform on Shimadzu UV-2401 PC $\mathrm{UV}-\mathrm{V}$ is Recording Spectrophotometer at a concentration of $10 \mu \mathrm{M}$. 


\section{Synthetic Details}

\subsection{Building blocks synthesis}

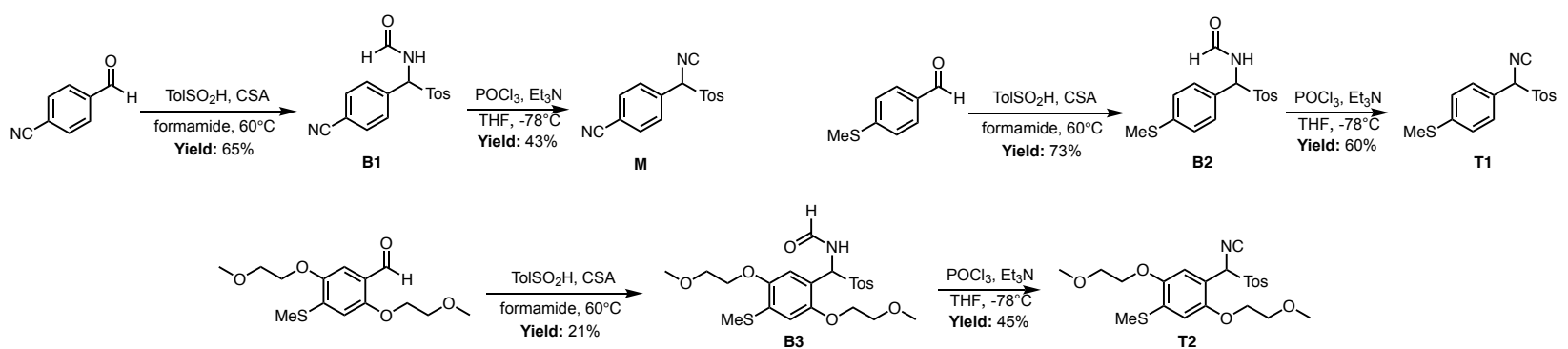

Figure S1: Synthesis of building blocks.<smiles>N#Cc1ccc(NC(=O)c2ccccc2)cc1</smiles>

N-((4-(methylthio)phenyl)(tosyl)methyl)formamide (B1). To a flask with a stir bar was added 4-(Methylthio)benzaldehyde (12.58 g, $94 \mathrm{mmol})$, p-Toluenesulfinic acid (12.48 g, $80 \mathrm{mmol})$, (1S)-Camphor-10-sulfonic acid (1.86 g, $8 \mathrm{mmol})$ and formamide $(20 \mathrm{~mL})$. The reaction mixture was stirred at $60^{\circ} \mathrm{C}$ for $24 \mathrm{~h}$. After cooling to room temperature, the mixture was diluted with $\mathrm{H}_{2} \mathrm{O}(400 \mathrm{~mL})$ and stirred for $30 \mathrm{~min}$. The resulting suspension was filtered to afford crude product as white solid $(16.435 \mathrm{~g}, 65 \%)$. The crude product was directly used for next step without any further purification.<smiles>CC([18OH])(c1ccc(C#N)cc1)c1ccc([N+]#N)cc1</smiles>

(4-(isocyano(tosyl)methyl)phenyl)(methyl)sulfane (M). To a stirred solution of B1 $(5 \mathrm{~g}, 15.9 \mathrm{mmol})$ in DME $(50 \mathrm{~mL})$ at $-40^{\circ} \mathrm{C}$ was slowly added $\mathrm{POCl}_{3}(4.43$ $\mathrm{mL}, 47.7 \mathrm{mmol})$. After stirring for $20 \mathrm{~min}$ at $-40^{\circ} \mathrm{C}, \mathrm{Et}_{3} \mathrm{~N}(11.05 \mathrm{~mL}, 79.5 \mathrm{mmol})$ was added dropwise. The resulting mixture was stirred at $-40^{\circ} \mathrm{C}$ for $2 \mathrm{~h}$ and quenched with saturated $\mathrm{NaHCO}_{3}$ solution. The product was extracted with $\mathrm{CH}_{2} \mathrm{Cl}_{2}$. The organic phase was dried $\left(\mathrm{MgSO}_{4}\right)$ and concentrated. Flash column chromatography $\left(\mathrm{SiO}_{2}, 20 \%-40 \%\right.$ ethyl acetate in hexanes) yielded the product as white solid $(2.02 \mathrm{~g}, 43 \%) .{ }^{1} \mathrm{H} \mathrm{NMR}\left(500 \mathrm{MHz}, \mathrm{CDCl}_{3}\right)$ $\delta 7.60-7.55(\mathrm{~m}, 2 \mathrm{H}), 7.28(\mathrm{~d}, J=7.9 \mathrm{~Hz}, 2 \mathrm{H}), 7.16(\mathrm{~s}, 4 \mathrm{H}), 5.48(\mathrm{~s}, 1 \mathrm{H}), 2.43(\mathrm{~s}, 3 \mathrm{H}), 2.41(\mathrm{~s}$, $3 \mathrm{H}) .{ }^{13} \mathrm{C} \mathrm{NMR}\left(125 \mathrm{MHz}, \mathrm{CDCl}_{3}\right) \delta 166.08,146.65,142.75,130.58,130.11,129.84,128.69$, 125.77, 122.60, 76.22,21.86, 15.13. HRMS (CI): Exact mass calcd for $\mathrm{C}_{16} \mathrm{H}_{13} \mathrm{~N}_{2} \mathrm{O}_{2} \mathrm{~S}[\mathrm{M}+\mathrm{H}]^{+}$ 296.07043, found 296.07055.<smiles>CSc1ccc(C(NC(=O)[O-])c2ccccc2)cc1</smiles>

N-((4-cyanophenyl)(tosyl)methyl)formamide (B2). To a flask with a stir bar was added 4-(Methylthio)benzaldehyde (1.0 g, $9.05 \mathrm{mmol})$, p-Toluenesulfinic acid (1.41 g, $9.05 \mathrm{mmol})$, (1S)-Camphor-10-sulfonic acid (211 mg, $0.91 \mathrm{mmol})$ and formamide $(10 \mathrm{~mL})$. The reaction mixture was stirred at $60^{\circ} \mathrm{C}$ for $24 \mathrm{~h}$. After cooling to room temperature, the mixture was diluted with $\mathrm{H}_{2} \mathrm{O}(400 \mathrm{~mL})$ and stirred for $30 \mathrm{~min}$. The resulting suspension was filtered to afford crude product as white solid $(2.22 \mathrm{~g}, 73 \%)$. The crude product was directly used for next step without any further purification.<smiles>CSc1ccc(C(C)(C)C)cc1</smiles>

4-(isocyano(tosyl)methyl)benzonitrile (T1). To a stirred solution of B2 (2 g, $5.97 \mathrm{mmol})$ in THF $(50 \mathrm{~mL})$ at $-78^{\circ} \mathrm{C}$ was slowly added $\mathrm{POCl}_{3}(1.67 \mathrm{~mL}, 17.9$ $\mathrm{mmol})$. After stirring for $20 \mathrm{~min}$ at $-78^{\circ} \mathrm{C}, \mathrm{Et}_{3} \mathrm{~N}(4.15 \mathrm{~mL}, 29.9 \mathrm{mmol})$ was added dropwise. The resulting mixture was stirred at $-40^{\circ} \mathrm{C}$ for $2 \mathrm{~h}$ and quenched with saturated $\mathrm{NaHCO}_{3}$ solution. The product was extracted with $\mathrm{CH}_{2} \mathrm{Cl}_{2}$. The organic phase was dried $\left(\mathrm{MgSO}_{4}\right)$ and concentrated. Flash column chromatography $\left(\mathrm{SiO}_{2}, 20 \%-40 \%\right.$ ethyl acetate in 
hexanes) yielded the product as white solid $(1.14 \mathrm{~g}, 60 \%) .{ }^{1} \mathrm{H}$ NMR $\left(500 \mathrm{MHz}, \mathrm{CDCl}_{3}\right) \delta 7.74(\mathrm{~d}$, $J=8.4 \mathrm{~Hz}, 2 \mathrm{H}), 7.66(\mathrm{~d}, J=8.3 \mathrm{~Hz}, 2 \mathrm{H}), 7.53(\mathrm{~d}, J=8.3 \mathrm{~Hz}, 2 \mathrm{H}), 7.40(\mathrm{~d}, J=8.1 \mathrm{~Hz}, 2 \mathrm{H}), 5.68$ $(\mathrm{s}, 1 \mathrm{H}), 2.52(\mathrm{~s}, 3 \mathrm{H}) .{ }^{13} \mathrm{C}$ NMR $(126 \mathrm{MHz}, \mathrm{CDCl} 3) \delta 166.08,146.65,142.75,130.58,130.11$, $129.84,128.69,125.77,125.68,122.60,21.86,15.13$. No mass spectra could be obtained by CI, EI or ESI.

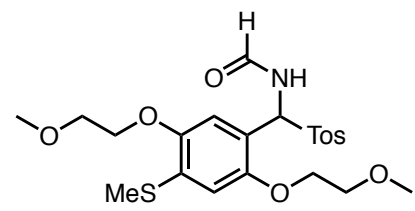

N-((4-(methylthio)phenyl)(tosyl)methyl)formamide (B3). To a flask with a stir bar was added 2,5-bis(2-methoxyethoxy)-4(methylthio)benzaldehyde (300 mg, $1 \mathrm{mmol}$ ), p-Toluenesulfinic acid (156 mg, $1 \mathrm{mmol})$, (1S)-Camphor-10-sulfonic acid (23 mg, $0.1 \mathrm{mmol}$ ) and formamide $(5 \mathrm{~mL})$. The reaction mixture was stirred at $60^{\circ} \mathrm{C}$ for 24 h. After cooling to room temperature, the mixture was diluted with $\mathrm{H}_{2} \mathrm{O}(100 \mathrm{~mL})$ and stirred for $30 \mathrm{~min}$. The resulting suspension was filtered to afford crude product as white solid (100 mg, 21\%). The crude product was directly used for next step without any further purification.

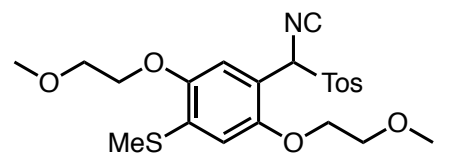

(4-(isocyano(tosyl)methyl)phenyl)(methyl)sulfane (T2). To a stirred solution of $\mathbf{B 3}(200 \mathrm{mg}, 0.21 \mathrm{mmol})$ in THF $(10 \mathrm{~mL})$ at $-78^{\circ} \mathrm{C}$ was slowly added $\mathrm{POCl}_{3}(0.059 \mathrm{~mL}, 0.63 \mathrm{mmol})$. After stirring for 20 min at $-78^{\circ} \mathrm{C}, \mathrm{Et}_{3} \mathrm{~N}(0.145 \mathrm{~mL}, 1.1 \mathrm{mmol})$ was added dropwise. The resulting mixture was stirred at $-78^{\circ} \mathrm{C}$ for $2 \mathrm{~h}$ and quenched with saturated $\mathrm{NaHCO}_{3}$ solution. The product was extracted with $\mathrm{CH}_{2} \mathrm{Cl}_{2}$. The organic phase was dried $\left(\mathrm{MgSO}_{4}\right)$ and concentrated. The crude product was directly used for next step without any further purification.

\section{S.2.2 Intermediate, control compound and sequence-defined oligomers synthesis}

\section{General DIBAL-H reduction procedure}

To a stirred solution of nitrile (1 equiv.) in $\mathrm{CH}_{2} \mathrm{Cl}_{2}$ at $-78^{\circ} \mathrm{C}$ was slowly added a $1.0 \mathrm{M}$ solution of DIBAL-H in hexane (1.2 equiv.). After stirring for $2 \mathrm{~h}$ at $-78^{\circ} \mathrm{C}$, the reaction mixture was quenched with saturated Rochelle salt solution. The product was extracted three times with $\mathrm{CH}_{2} \mathrm{Cl}_{2}$. The combined organic extracts were dried $\left(\mathrm{MgSO}_{4}\right)$ and concentrated. Flash column chromatography $\left(\mathrm{SiO}_{2}\right.$, ethyl acetate in hexanes) yielded the product.

\section{General van Leusen oxazole synthesis procedure ${ }^{7,8}$}

To a flask with a stir bar was added aldehyde (1 equiv.), isonitrile (1 equiv.), $\mathrm{K}_{2} \mathrm{CO}_{3}$ (3 equiv.), and $\mathrm{MeOH} / \mathrm{DME}(3: 1)$. The reaction mixture was stirred at $60^{\circ} \mathrm{C}$ for $16 \mathrm{~h}$. After cooling to room temperature, the mixture was diluted with $\mathrm{H}_{2} \mathrm{O}$ and extracted three times with $\mathrm{CH}_{2} \mathrm{Cl}_{2}$. The combined organic extracts were dried $\left(\mathrm{MgSO}_{4}\right)$ and concentrated. Flash column chromatography $\left(\mathrm{SiO}_{2}\right.$, ethyl acetate in hexanes) yielded the product.

\section{General van Leusen imidazole synthesis procedure}

To a flask with a stir bar was added aldehyde (1 equiv.), octylamine (4 equiv.) and $\mathrm{MeOH}$. The reaction mixture was stirred at room temperature for $1 \mathrm{~h}$. Then, $\mathrm{K}_{2} \mathrm{CO}_{3}$ (3 equiv.) and solution of isonitrile (1 equiv.) in DME were added to the reaction mixture. The resulting mixture was stirred at $60^{\circ} \mathrm{C}$ for $16 \mathrm{~h}$. After cooling to room temperature, the mixture was diluted with $\mathrm{H}_{2} \mathrm{O}$ and extracted three times with $\mathrm{CH}_{2} \mathrm{Cl}_{2}$. The combined organic extracts were dried $\left(\mathrm{MgSO}_{4}\right)$ and concentrated. Flash column chromatography $\left(\mathrm{SiO}_{2}\right.$, ethyl acetate in hexanes) yielded the product. 


\section{General van Leusen pyrrole synthesis procedure}

To a stirred solution of isonitrile (1 equiv.) in THF at $-40^{\circ} \mathrm{C}$ was slowly added a $1.6 \mathrm{M}$ solution of $\mathrm{n}$-BuLi in hexane (1.1 equiv.). After stirring for $20 \mathrm{~min}$ at $-40^{\circ} \mathrm{C}, \mathrm{LiBr}(6$ equiv.) and alkane (1 equiv.) were added to the reaction mixture slowly. The reaction mixture was stirred at $-40^{\circ} \mathrm{C}$ for 2 $\mathrm{h}$ and quenched with saturated $\mathrm{NH}_{4} \mathrm{OAc}$ solution. The product was extracted three times with $\mathrm{CH}_{2} \mathrm{Cl}_{2}$. The combined organic extract was dried $\left(\mathrm{MgSO}_{4}\right)$ and concentrated. Flash column chromatography $\left(\mathrm{SiO}_{2}\right.$, ethyl acetate in hexanes) yielded the product.
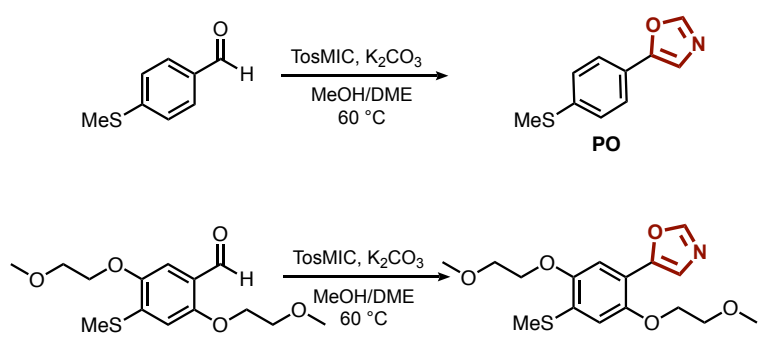

GO
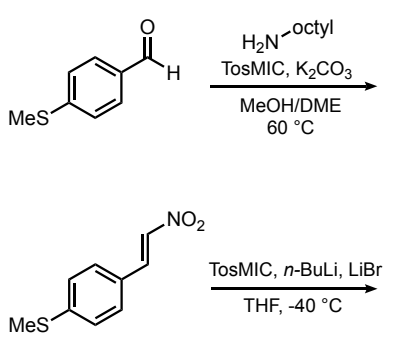<smiles>Cc1ccc(-c2cncn2N)cc1</smiles><smiles>Cc1ccc(-c2c[nH]cc2N=O)cc1</smiles>

Figure S2: Synthesis of dimers.
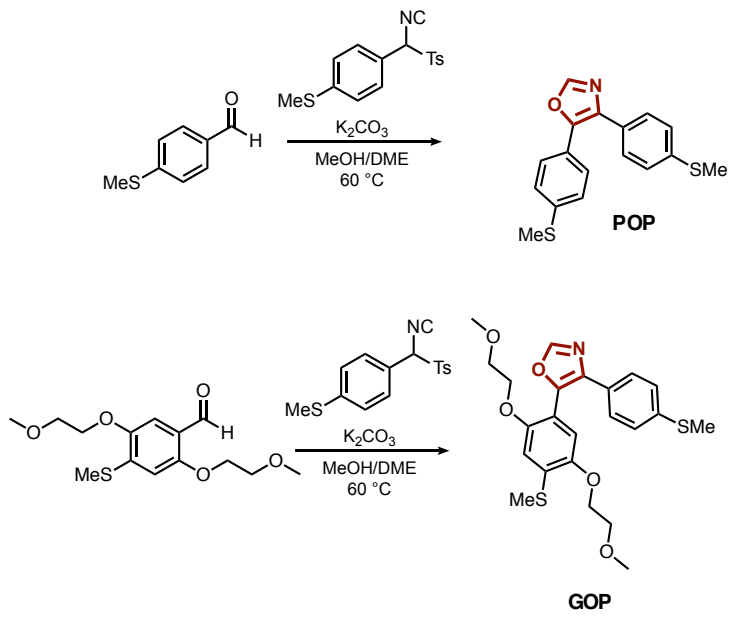

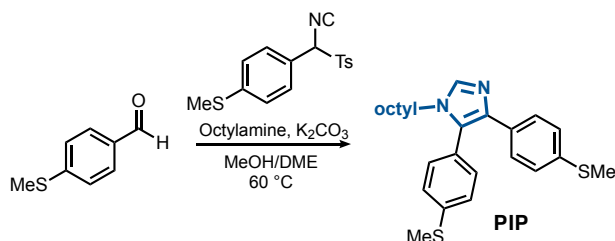<smiles>COc1ccc(-c2[nH]cc(-c3ccc(S(C)(=O)=O)cc3)c2-c2ccc(S(=O)(=O)c3ccc(C(F)(F)F)cc3)cc2)cc1</smiles>

Figure S3: Synthesis of trimers. 


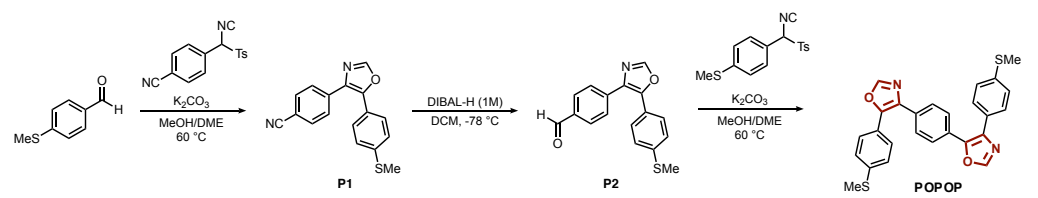

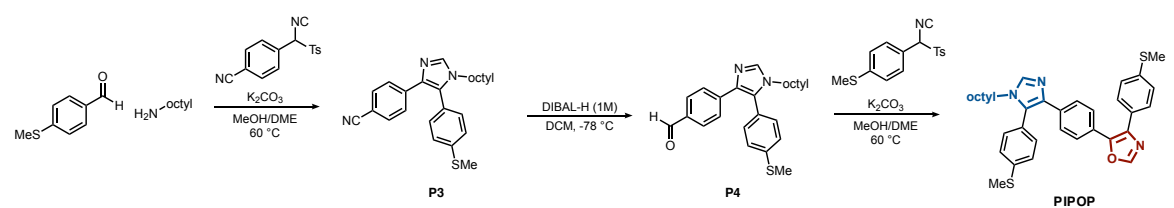

$$
\begin{aligned}
& \text {. }
\end{aligned}
$$

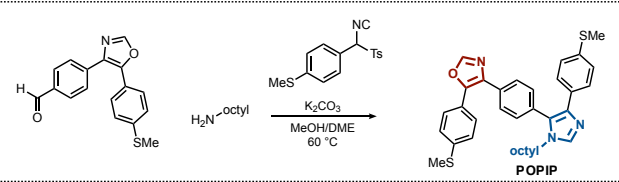

$$
\begin{aligned}
& \text { ore } \\
& \text { (n) } \\
& \text { (100) } \\
& \text { (⿸t) } \\
& \text { 象 } \\
& \text { (n) }
\end{aligned}
$$

Figure S4: Synthesis of sequence-defined pentamers. 


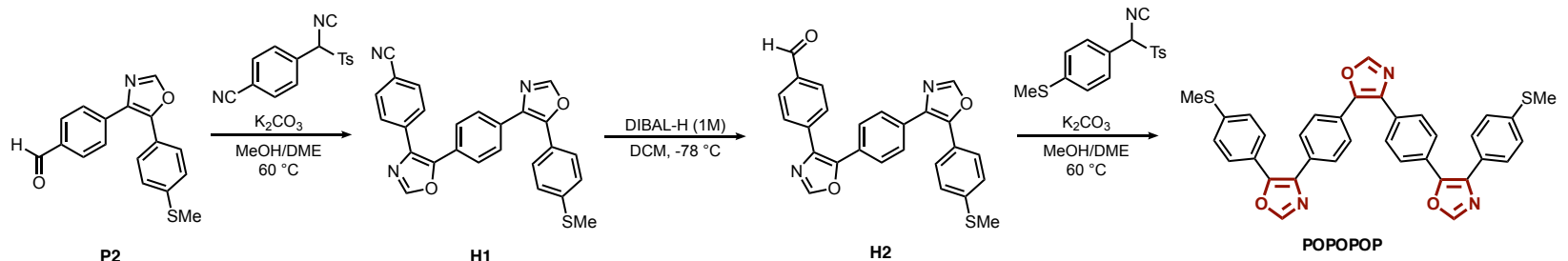

Figure S5: Synthesis of sequence-defined heptamer.

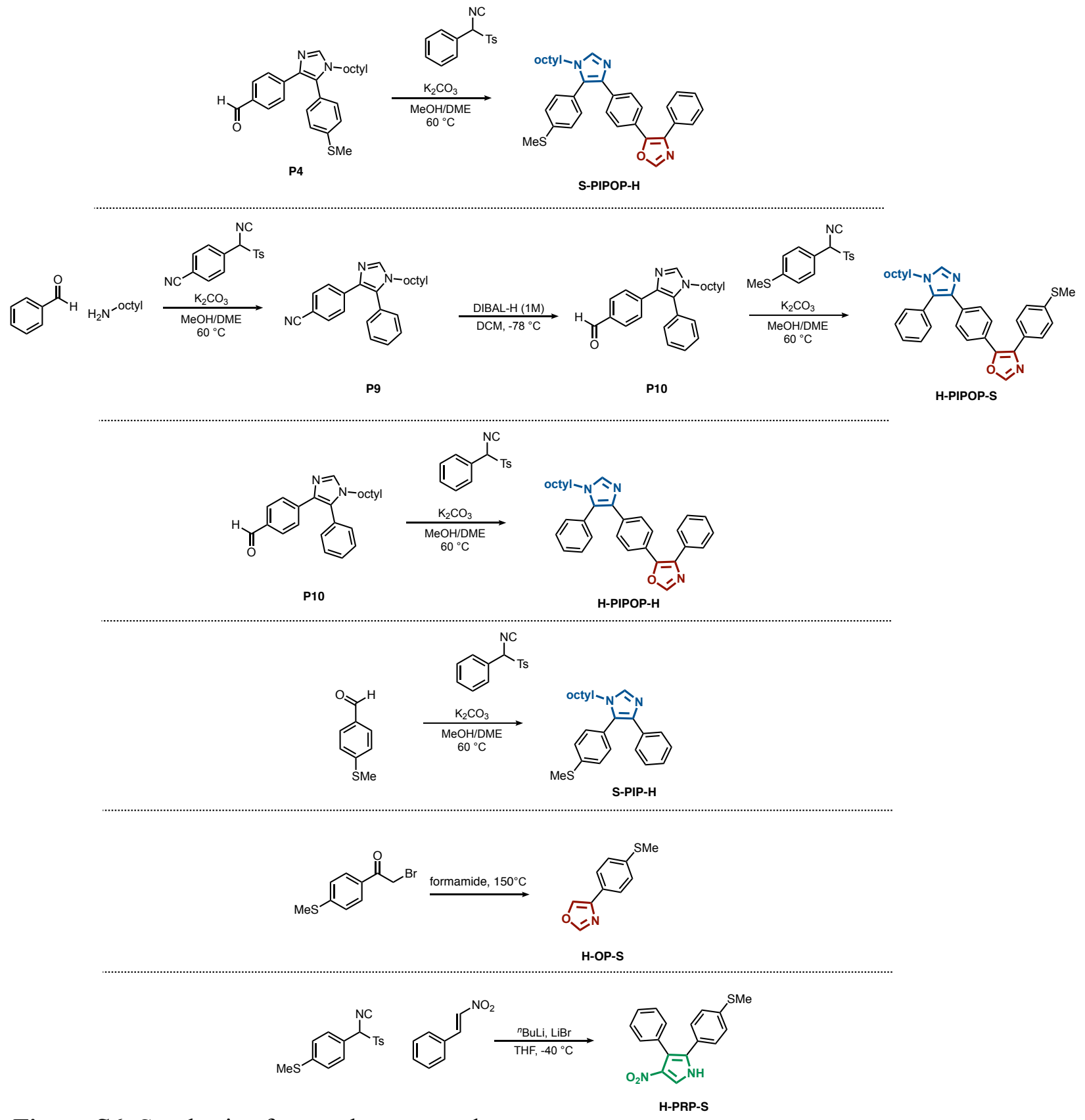

Figure S6: Synthesis of control compounds. 


\subsection{Intermediate synthesis}

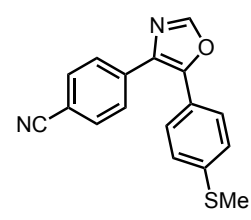

4-(5-(4-(methylthio)phenyl)oxazol-4-yl)benzonitrile (P1). Prepared according to the general van Leusen imidazole synthesis procedure using 4(Methylthio)benzaldehyde (0.1 mL, $0.75 \mathrm{mmol}), \mathbf{M}(266 \mathrm{mg}, 0.9 \mathrm{mmol}), \mathrm{K}_{2} \mathrm{CO}_{3}$ (322 mg, $2.25 \mathrm{mmol}), \mathrm{MeOH}(3 \mathrm{~mL})$ and DME $(1 \mathrm{~mL})$. White solid (180 $\mathrm{mg}$, 82\%). ${ }^{1} \mathrm{H}$ NMR $\left(500 \mathrm{MHz}, \mathrm{CDCl}_{3}\right) \delta 7.99(\mathrm{~s}, 1 \mathrm{H}), 7.82(\mathrm{~d}, J=8.4 \mathrm{~Hz}, 2 \mathrm{H}), 7.68$ $(\mathrm{d}, J=8.4 \mathrm{~Hz}, 2 \mathrm{H}), 7.51(\mathrm{~d}, J=8.5 \mathrm{~Hz}, 2 \mathrm{H}), 7.31(\mathrm{~s}, 2 \mathrm{H}), 2.55(\mathrm{~s}, 3 \mathrm{H}) .{ }^{13} \mathrm{C} \mathrm{NMR}(125 \mathrm{MHz}$, $\left.\mathrm{CDCl}_{3}\right) \delta 150.03,147.28,141.19,136.76,132.69,132.44,128.04,127.49,126.13,124.24,118.76$, 111.51, 15.17. HRMS (ESI): Exact mass calcd for $\mathrm{C}_{17} \mathrm{H}_{13} \mathrm{~N}_{2} \mathrm{OS}[\mathrm{M}+\mathrm{H}]^{+}$293.0749, found 293.0746.

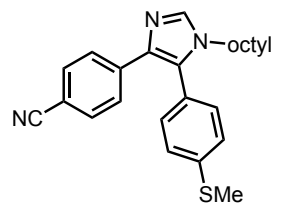

4-(5-(4-(methylthio)phenyl)-1-octyl-1H-imidazol-4-yl)benzonitrile (P3). Prepared according to the general van Leusen imidazole synthesis procedure using 4-(Methylthio)benzaldehyde (0.13 mL, $1 \mathrm{mmol}), \mathbf{M}(355 \mathrm{mg}, 1.2 \mathrm{mmol})$, octylamine (0.2 mL, $1.2 \mathrm{mmol}), \mathrm{K}_{2} \mathrm{CO}_{3}(419 \mathrm{mg}, 3 \mathrm{mmol}), \mathrm{MeOH}(6 \mathrm{~mL})$ and DME (2 mL). White solid (305 mg, 75\%). ${ }^{1} \mathrm{H}$ NMR $\left(500 \mathrm{MHz}, \mathrm{CDCl}_{3}\right) \delta 7.64$ $(\mathrm{s}, 1 \mathrm{H}), 7.60(\mathrm{~d}, J=8.7 \mathrm{~Hz}, 2 \mathrm{H}), 7.50(\mathrm{~d}, J=8.7 \mathrm{~Hz}, 2 \mathrm{H}), 7.37(\mathrm{~d}, J=8.4 \mathrm{~Hz}, 2 \mathrm{H}), 7.25(\mathrm{~d}, J=$ $8.5 \mathrm{~Hz}, 2 \mathrm{H}), 3.79(\mathrm{t}, J=7.4 \mathrm{~Hz}, 2 \mathrm{H}), 2.59(\mathrm{~s}, 3 \mathrm{H}), 1.60(\mathrm{~d}, J=8.9 \mathrm{~Hz}, 2 \mathrm{H}), 1.21(\mathrm{~s}, 9 \mathrm{H}), 0.89$ (t, $J$ $=7.1 \mathrm{~Hz}, 3 \mathrm{H}) .{ }^{13} \mathrm{C}$ NMR $\left(125 \mathrm{MHz}, \mathrm{CDCl}_{3}\right) \delta 140.52,139.28,137.21,136.41,132.06,130.87$, 129.96, 126.49, 126.26, 119.38, 109.22, 45.23, 31.69, 30.80, 29.00, 28.89, 26.43, 22.59, 15.13, 14.07. HRMS (ESI): Exact mass calcd for $\mathrm{C}_{25} \mathrm{H}_{30} \mathrm{~N}_{3} \mathrm{~S}[\mathrm{M}+\mathrm{H}]^{+} 404.2160$, found 404.2155.

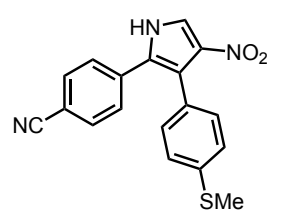

4-(3-(4-(methylthio)phenyl)-4-nitro-1H-pyrrol-2-yl)benzonitrile

(P5).

Prepared according to the general van Leusen pyrrole synthesis procedure using B2 (78 mg, $0.4 \mathrm{mmol}), \mathbf{M}(130 \mathrm{mg}, 0.44 \mathrm{mmol}), 1.6 \mathrm{M} \mathrm{n}$-BuLi in Hexane (0.275 $\mathrm{mL}, 0.44 \mathrm{mmol}), \mathrm{LiBr}(208 \mathrm{mg}, 2.4 \mathrm{mmol})$ and THF (10 mL). Yellow solid (100 mg, 75\%). ${ }^{1} \mathrm{H}$ NMR $\left(500 \mathrm{MHz}, \mathrm{CDCl}_{3}\right) \delta 8.81(\mathrm{~s}, 1 \mathrm{H}), 7.91(\mathrm{~d}, J=3.6 \mathrm{~Hz}, 1 \mathrm{H})$, $7.58(\mathrm{~d}, J=8.6 \mathrm{~Hz}, 2 \mathrm{H}), 7.26(\mathrm{~d}, J=6.8 \mathrm{~Hz}, 4 \mathrm{H}), 7.20(\mathrm{~d}, J=8.4 \mathrm{~Hz}, 2 \mathrm{H}), 2.54(\mathrm{~s}, 3 \mathrm{H}) .{ }^{13} \mathrm{C} \mathrm{NMR}$ $\left(125 \mathrm{MHz} \mathrm{CDCl}_{3}\right) \delta 138.82,136.84,135.11,132.66,130.89,128.28,127.47,127.43,126.09$, 121.36, 119.14, 118.29, 111.40, 15.37. HRMS (ESI): Exact mass calcd for $\mathrm{C}_{18} \mathrm{H}_{13} \mathrm{~N}_{3} \mathrm{O}_{2} \mathrm{~S}[\mathrm{M}+\mathrm{H}]^{+}$ 335.0728, found 335.0724.

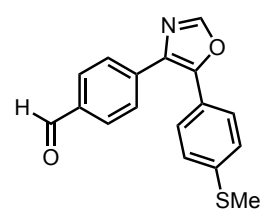

4-(5-(4-(methylthio)phenyl)oxazol-4-yl)benzaldehyde (P2). Prepared according to the general DIBAL-H reduction procedure using P1 (350 mg, 1.18 mmol), 1M DIBAL-H (1.42 mL, $1.42 \mathrm{mmol})$ and $\mathrm{CH}_{2} \mathrm{Cl}_{2}(5 \mathrm{~mL})$. White solid (200 mg, 90\%). ${ }^{1} \mathrm{H}$ NMR $\left(500 \mathrm{MHz}, \mathrm{CDCl}_{3}\right) \delta 10.05(\mathrm{~s}, 1 \mathrm{H}), 8.00(\mathrm{~s}, 1 \mathrm{H}), 7.90$ $(\mathrm{q}, J=8.3 \mathrm{~Hz}, 4 \mathrm{H}), 7.54(\mathrm{~d}, J=8.6 \mathrm{~Hz}, 2 \mathrm{H}), 7.30(\mathrm{~s}, 2 \mathrm{H}), 2.55(\mathrm{~s}, 3 \mathrm{H}) .{ }^{13} \mathrm{C} \mathrm{NMR}$ $\left(125 \mathrm{MHz}, \mathrm{CDCl}_{3}\right) \delta 191.68,149.96,147.18,140.92,138.22,135.73,133.27,130.05,128.10$, 127.46, 126.11, 124.49, 15.21. HRMS (ESI): Exact mass calcd for $\mathrm{C}_{17} \mathrm{H}_{14} \mathrm{NO}_{2} \mathrm{~S}[\mathrm{M}+\mathrm{H}]^{+} 296.0745$, found 296.0745 .

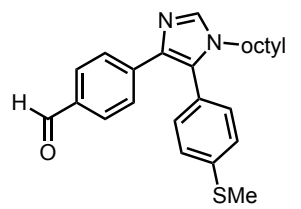

4-(5-(4-(methylthio)phenyl)-1-octyl-1H-imidazol-4-yl)benzaldehyde (P4). Prepared according to the general DIBAL-H reduction procedure using P3 (300 $\mathrm{mg}, 0.75 \mathrm{mmol}), 1 \mathrm{M}$ DIBAL-H $(0.9 \mathrm{~mL}, 0.9 \mathrm{mmol})$ and $\mathrm{CH}_{2} \mathrm{Cl}_{2}(10 \mathrm{~mL})$. White solid (150 mg, 50\%). ${ }^{1} \mathrm{H}$ NMR $\left(500 \mathrm{MHz}, \mathrm{CDCl}_{3}\right) \delta 9.94(\mathrm{~s}, 1 \mathrm{H}), 7.74(\mathrm{~d}, J=$ $8.4 \mathrm{~Hz}, 2 \mathrm{H}), 7.68(\mathrm{~s}, 1 \mathrm{H}), 7.66(\mathrm{~d}, J=3.0 \mathrm{~Hz}, 2 \mathrm{H}), 7.37(\mathrm{~d}, J=8.3 \mathrm{~Hz}, 2 \mathrm{H})$, $7.26(\mathrm{~d}, J=8.3 \mathrm{~Hz}, 2 \mathrm{H}), 3.80(\mathrm{t}, J=7.3 \mathrm{~Hz}, 2 \mathrm{H}), 2.58(\mathrm{~s}, 3 \mathrm{H}), 1.64-1.58(\mathrm{~m}, 2 \mathrm{H}), 1.28(\mathrm{q}, J=$ 
$6.9 \mathrm{~Hz}, 2 \mathrm{H}), 1.22(\mathrm{~s}, 8 \mathrm{H}), 0.89(\mathrm{t}, J=7.1 \mathrm{~Hz}, 3 \mathrm{H}) .{ }^{13} \mathrm{C} \mathrm{NMR}\left(125 \mathrm{MHz}, \mathrm{CDCl}_{3}\right) \delta 191.91,140.90$, 140.33 , 137.22, 136.97, 134.15, 130.94, 130.03, 129.85, 126.53, 126.50, 126.47, 45.23, 31.69, 30.81, 29.00, 28.90, 26.44, 22.59, 15.18, 14.07. HRMS (ESI): Exact mass calcd for $\mathrm{C}_{25} \mathrm{H}_{30} \mathrm{~N}_{2} \mathrm{OS}$ $[\mathrm{M}+\mathrm{H}]^{+}$407.2157, found 407.2137.

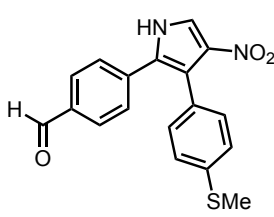

4-(3-(4-(methylthio)phenyl)-4-nitro-1H-pyrrol-2-yl)benzaldehyde

(P6). Prepared according to the general DIBAL-H reduction procedure using P5 (392 $\mathrm{mg}, 1.35 \mathrm{mmol}), 1 \mathrm{M}$ DIBAL-H $(1.62 \mathrm{~mL}, 1.62 \mathrm{mmol})$ and $\mathrm{CH}_{2} \mathrm{Cl}_{2}(5 \mathrm{~mL})$. Brown solid (173 mg, 38\%). ${ }^{1} \mathrm{H}$ NMR $\left(500 \mathrm{MHz}, \mathrm{CDCl}_{3}\right) \delta 9.98(\mathrm{~s}, 1 \mathrm{H}), 8.85$ $(\mathrm{s}, 1 \mathrm{H}), 7.91(\mathrm{~s}, 1 \mathrm{H}), 7.81(\mathrm{~d}, J=8.4 \mathrm{~Hz}, 2 \mathrm{H}), 7.33(\mathrm{~d}, J=8.3 \mathrm{~Hz}, 2 \mathrm{H}), 7.27(\mathrm{~d}$, $J=8.6 \mathrm{~Hz}, 2 \mathrm{H}), 7.23(\mathrm{~d}, J=8.5 \mathrm{~Hz}, 2 \mathrm{H}), 2.54(\mathrm{~s}, 3 \mathrm{H}) .{ }^{13} \mathrm{C} \mathrm{NMR}\left(125 \mathrm{MHz}, \mathrm{CDCl}_{3}\right) \delta 191.27$, 138.58, 136.47, 135.27, 130.98, 130.21, 128.97, 127.79, 127.41, 126.10, 121.23, 118.96, 15.43. HRMS (ESI): Exact mass calcd for $\mathrm{C}_{18} \mathrm{H}_{14} \mathrm{~N}_{2} \mathrm{O}_{3} \mathrm{~S}[\mathrm{M}]^{+} 338.0725$, found 338.0717.

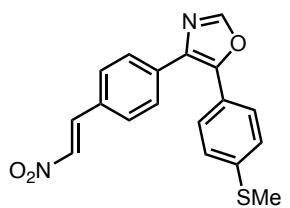

(E)-5-(4-(methylthio)phenyl)-4-(4-(2-nitrovinyl)phenyl)oxazole (P7). To a flask with a stir bar was added P2 (100 $\mathrm{mg}, 0.34 \mathrm{mmol})$, nitromethane (140 $\mathrm{mg}$, $2.3 \mathrm{mmol}), \mathrm{NH}_{4} \mathrm{OAc}(63 \mathrm{mg}, 0.816 \mathrm{mmol})$ and $\mathrm{HOAc}(10 \mathrm{~mL})$. The reaction mixture was stirred at $100^{\circ} \mathrm{C}$ for $16 \mathrm{~h}$. After cooling to room temperature, the mixture was diluted with $\mathrm{H}_{2} \mathrm{O}$ and extracted three times with EtOAc. The combined organic extracts were dried $\left(\mathrm{MgSO}_{4}\right)$ and concentrated. Flash column chromatography $\left(\mathrm{SiO}_{2}, 20 \%-40 \%\right.$ ethyl acetate in hexanes) yielded the product as an orange solid $(80 \mathrm{mg}, 70 \%)$. ${ }^{1} \mathrm{H} \mathrm{NMR}\left(500 \mathrm{MHz}, \mathrm{CDCl}_{3}\right) \delta 8.03(\mathrm{~d}, J=13.9 \mathrm{~Hz}, 1 \mathrm{H}), 7.99(\mathrm{~s}, 1 \mathrm{H}), 7.80(\mathrm{~d}, J=8.3 \mathrm{~Hz}, 2 \mathrm{H})$, $7.63(\mathrm{~d}, J=13.7 \mathrm{~Hz}, 1 \mathrm{H}), 7.58(\mathrm{~d}, J=8.3 \mathrm{~Hz}, 2 \mathrm{H}), 7.53$ (d, $J=8.5 \mathrm{~Hz}, 2 \mathrm{H}), 7.30(\mathrm{~s}, 2 \mathrm{H}), 2.55(\mathrm{~s}$, $3 \mathrm{H}) .{ }^{13} \mathrm{C} \mathrm{NMR}\left(125 \mathrm{MHz}, \mathrm{CDCl}_{3}\right) \delta 149.95,146.91,140.88,138.48,137.10,136.05,133.23$, 129.61, 129.48, 128.40, 127.45, 126.11, 124.56, 15.21. HRMS (ESI): Exact mass calcd for $\mathrm{C}_{18} \mathrm{H}_{15} \mathrm{~N}_{2} \mathrm{O}_{3} \mathrm{~S}[\mathrm{M}+\mathrm{H}]^{+} 339.0803$, found 339.0800.

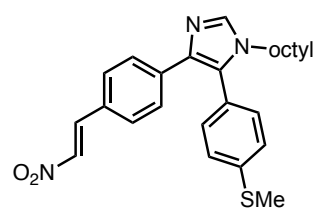

(E)-5-(4-(methylthio)phenyl)-4-(4-(2-nitrovinyl)phenyl)-1-octyl-1Himidazole (P8). To a flask with a stir bar was added P4 (80 mg, $0.20 \mathrm{mmol})$, nitromethane ( $84 \mathrm{mg}, 1.38 \mathrm{mmol}), \mathrm{NH}_{4} \mathrm{OAc}(40 \mathrm{mg}, 0.48 \mathrm{mmol})$ and $\mathrm{HOAc}$ $(5 \mathrm{~mL})$. The reaction mixture was stirred at $100^{\circ} \mathrm{C}$ for $16 \mathrm{~h}$. After cooling to room temperature, the mixture was diluted with $\mathrm{H}_{2} \mathrm{O}$ and extracted three

times with EtOAc. The combined organic extracts were dried $\left(\mathrm{MgSO}_{4}\right)$ and concentrated. Flash column chromatography $\left(\mathrm{SiO}_{2}, 20 \%-40 \%\right.$ ethyl acetate in hexanes) yielded the product as an Yellow solid (60 mg, 67\%). ${ }^{1} \mathrm{H}$ NMR $\left(500 \mathrm{MHz}, \mathrm{CDCl}_{3}\right) \delta 7.97(\mathrm{~d}, J=13.6 \mathrm{~Hz}, 1 \mathrm{H}), 7.66(\mathrm{~s}, 1 \mathrm{H})$, $7.59(\mathrm{~d}, J=8.5 \mathrm{~Hz}, 2 \mathrm{H}), 7.56(\mathrm{~d}, J=13.6 \mathrm{~Hz}, 1 \mathrm{H}), 7.50(\mathrm{~d}, J=8.6 \mathrm{~Hz}, 1 \mathrm{H}), 7.42(\mathrm{~d}, J=8.4 \mathrm{~Hz}$, $1 \mathrm{H}), 7.37(\mathrm{~d}, J=8.3 \mathrm{~Hz}, 2 \mathrm{H}), 7.28-7.22(\mathrm{~m}, 2 \mathrm{H}), 3.80$ (t, $J=7.3 \mathrm{~Hz}, 2 \mathrm{H}), 2.59(\mathrm{~s}, 3 \mathrm{H}), 1.60(\mathrm{~m}$, $J=6.9 \mathrm{~Hz}, 2 \mathrm{H}), 1.22(\mathrm{~m}, 10 \mathrm{H}), 0.89$ (t, $J=7.1 \mathrm{~Hz}, 3 \mathrm{H}) .{ }^{13} \mathrm{C} \mathrm{NMR}\left(125 \mathrm{MHz}, \mathrm{CDCl}_{3}\right) \delta 140.35$, $139.18,138.86,137.17,136.90,136.07,132.07,130.95,130.87,129.72,129.34,127.60,126.90$, 126.50, 126.48, 126.45, 45.24, 31.69, 30.81, 29.00, 28.90, 26.44, 22.59, 15.15, 15.12, 14.07. HRMS (ESI): Exact mass calcd for $\mathrm{C}_{26} \mathrm{H}_{32} \mathrm{~N}_{3} \mathrm{O}_{2} \mathrm{~S}[\mathrm{M}+\mathrm{H}]^{+} 450.2215$, found 450.2207.

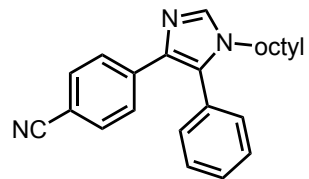

4-(1-octyl-5-phenyl-1H-imidazol-4-yl)benzonitrile

(P9). Prepared according to the general van Leusen imidazole synthesis procedure using benzaldehyde $(0.2 \mathrm{~mL}, 2 \mathrm{mmol}), \mathbf{M}(500 \mathrm{mg}, 1.7 \mathrm{mmol})$, octylamine $(1.7 \mathrm{~mL}$, $10 \mathrm{mmol}), \mathrm{K}_{2} \mathrm{CO}_{3}(800 \mathrm{mg}, 6 \mathrm{mmol}), \mathrm{MeOH}(9 \mathrm{~mL})$ and $\mathrm{DME}(3 \mathrm{~mL})$. Colorless oil (450 mg, 75\%). ${ }^{1} \mathrm{H}$ NMR $\left(500 \mathrm{MHz}, \mathrm{CDCl}_{3}\right) \delta 7.65(\mathrm{~s}, 1 \mathrm{H}), 7.57(\mathrm{~d}, J=8.4 \mathrm{~Hz}, 2 \mathrm{H})$, $7.54-7.51(\mathrm{~m}, 3 \mathrm{H}), 7.49(\mathrm{~s}, 2 \mathrm{H}), 7.34(\mathrm{dd}, J=6.5,3.0 \mathrm{~Hz}, 2 \mathrm{H}), 3.83-3.76(\mathrm{~m}, 2 \mathrm{H}), 1.61-1.57$ 
$(\mathrm{m}, 2 \mathrm{H}), 1.30-1.26(\mathrm{~m}, 2 \mathrm{H}), 1.20(\mathrm{~s}, 8 \mathrm{H}), 0.88(\mathrm{t}, J=7.1 \mathrm{~Hz}, 3 \mathrm{H}) .{ }^{13} \mathrm{C} \mathrm{NMR}\left(125 \mathrm{MHz}, \mathrm{CDCl}_{3}\right)$ $\delta 139.30,137.14,136.29,132.01,130.60,130.49,130.23,129.38,129.29,126.73,126.48,119.38$, $109.17,45.26,31.73,31.66,30.74,29.11,28.96,28.89,28.85,26.40,22.61,22.58,14.06$. HRMS (ESI): Exact mass calcd for $\mathrm{C}_{24} \mathrm{H}_{28} \mathrm{~N}_{3}[\mathrm{M}+\mathrm{H}]^{+} 358.2283$, found 358.2272.

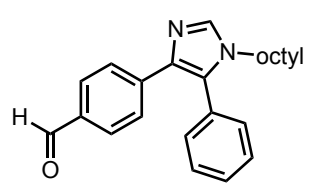

4-(1-octyl-5-phenyl-1H-imidazol-4-yl)benzaldehyde $\quad$ (P10). Prepared according to the general DIBAL-H reduction procedure using $\mathbf{P 9}(300 \mathrm{mg}$, $0.75 \mathrm{mmol}), 1 \mathrm{M}$ DIBAL-H $(0.9 \mathrm{~mL}, 0.9 \mathrm{mmol})$ and $\mathrm{CH}_{2} \mathrm{Cl}_{2}(10 \mathrm{~mL})$. White solid (150 mg, 50\%). ${ }^{1} \mathrm{H}$ NMR $\left(500 \mathrm{MHz}, \mathrm{CDCl}_{3}\right) \delta 9.93(\mathrm{~s}, 1 \mathrm{H}), 7.73(\mathrm{~d}, J=$ $8.2 \mathrm{~Hz}, 2 \mathrm{H}), 7.67-7.63(\mathrm{~m}, 3 \mathrm{H}), 7.55-7.51(\mathrm{~m}, 3 \mathrm{H}), 7.36(\mathrm{dd}, J=6.6,2.5 \mathrm{~Hz}, 2 \mathrm{H}), 3.81(\mathrm{t}, J=$ $7.4 \mathrm{~Hz}, 2 \mathrm{H}), 1.24(\mathrm{~d}, J=37.4 \mathrm{~Hz}, 10 \mathrm{H}), 0.88(\mathrm{t}, J=7.1 \mathrm{~Hz}, 3 \mathrm{H}) .{ }^{13} \mathrm{C} \mathrm{NMR}\left(125 \mathrm{MHz}, \mathrm{CDCl}_{3}\right) \delta$ $191.93,140.93$, 137.14, 136.86, 134.12, 130.67, 130.57, 130.43, 129.82, 129.30, 129.18, 126.51, 45.26, 31.67, 30.75, 28.97, 28.86, 26.41, 22.58, 14.06. HRMS (ESI): Exact mass calcd for $\mathrm{C}_{24} \mathrm{H}_{29} \mathrm{~N}_{2} \mathrm{O}[\mathrm{M}+\mathrm{H}]^{+}$361.2280, found 361.2275.

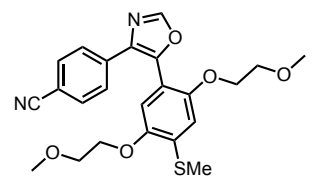

4-(5-(2,5-bis(2-methoxyethoxy)-4-(methylthio)phenyl)oxazol-4yl)benzonitrile (O1). Prepared according to the general van Leusen oxazole synthesis procedure using 2,5-bis(2-methoxyethoxy)-4(methylthio)benzaldehyde (100 mg, $0.33 \mathrm{mmol}), \mathbf{M}(110 \mathrm{mg}, 0.36 \mathrm{mmol})$, $\mathrm{K}_{2} \mathrm{CO}_{3}(138 \mathrm{mg}, 1 \mathrm{mmol}), \mathrm{MeOH}(3 \mathrm{~mL})$ and DME $(1 \mathrm{~mL})$. Yellow oil $(130 \mathrm{mg}, 89 \%)$. ${ }^{1} \mathrm{H} \mathrm{NMR}$ $\left(500 \mathrm{MHz}, \mathrm{CDCl}_{3}\right) \delta 8.01(\mathrm{~d}, J=3.6 \mathrm{~Hz}, 1 \mathrm{H}), 7.75-7.68(\mathrm{~m}, 2 \mathrm{H}), 7.62(\mathrm{dd}, J=9.0,2.4 \mathrm{~Hz}, 2 \mathrm{H})$, $6.98(\mathrm{~s}, 1 \mathrm{H}), 6.83(\mathrm{~s}, 1 \mathrm{H}), 4.17-4.11(\mathrm{~m}, 3 \mathrm{H}), 3.96-3.87(\mathrm{~m}, 2 \mathrm{H}), 3.83-3.74(\mathrm{~m}, 2 \mathrm{H}), 3.48(\mathrm{~d}$, $J=5.1 \mathrm{~Hz}, 3 \mathrm{H}), 3.19(\mathrm{~d}, J=8.4 \mathrm{~Hz}, 3 \mathrm{H}), 3.17-3.11(\mathrm{~m}, 2 \mathrm{H}), 2.50(\mathrm{~s}, 2 \mathrm{H}) .{ }^{13} \mathrm{C}$ NMR $(125 \mathrm{MHz}$, $\left.\mathrm{CDCl}_{3}\right) \delta 151.03,150.40,150.28,149.82,144.41,137.28,134.09,132.75,131.85,127.27,127.25$, $118.98,117.76,116.68,114.18,113.85,113.78,111.08,110.72,71.04,70.98,70.57,69.39,68.82$, $68.36,68.08,60.40,59.40,59.10,59.08,21.07,14.40,14.21$. HRMS (ESI): Exact mass calcd for $\mathrm{C}_{23} \mathrm{H}_{25} \mathrm{~N}_{2} \mathrm{O}_{5} \mathrm{~S}[\mathrm{M}+\mathrm{H}]^{+} 441.1484$, found 441.1485 .

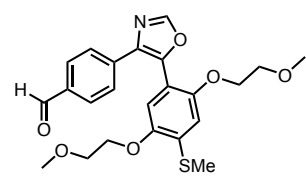

4-(5-(2,5-bis(2-methoxyethoxy)-4-(methylthio)phenyl)oxazol-4yl)benzaldehyde (O2). Prepared according to the general DIBAL-H reduction procedure using 01 (130 mg, $0.3 \mathrm{mmol}), 1 \mathrm{M}$ DIBAL-H $(0.33 \mathrm{~mL}, 0.33 \mathrm{mmol})$ and $\mathrm{CH}_{2} \mathrm{Cl}_{2}(10 \mathrm{~mL})$. Colorless oil $(30 \mathrm{mg}, 23 \%)$. ${ }^{1} \mathrm{H}$ NMR $\left(500 \mathrm{MHz}, \mathrm{CDCl}_{3}\right)$ $\delta 10.02(\mathrm{~s}, 1 \mathrm{H}), 8.02(\mathrm{~d}, J=3.6 \mathrm{~Hz}, 1 \mathrm{H}), 7.85(\mathrm{dd}, J=8.4,3.1 \mathrm{~Hz}, 2 \mathrm{H}), 7.78(\mathrm{dd}, J=8.3,5.5 \mathrm{~Hz}$, 2H), $7.00(\mathrm{~s}, 1 \mathrm{H}), 6.83(\mathrm{~s}, 1 \mathrm{H}), 4.16-4.11(\mathrm{~m}, 2 \mathrm{H}), 3.90(\mathrm{dt}, J=15.5,4.9 \mathrm{~Hz}, 2 \mathrm{H}), 3.81-3.74$ $(\mathrm{m}, 2 \mathrm{H}), 3.47(\mathrm{~d}, J=4.5 \mathrm{~Hz}, 3 \mathrm{H}), 3.16(\mathrm{~d}, J=8.7 \mathrm{~Hz}, 3 \mathrm{H}), 3.13-3.08(\mathrm{~m}, 2 \mathrm{H}), 2.50(\mathrm{~s}, 2 \mathrm{H}) .{ }^{13} \mathrm{C}$ NMR $\left(125 \mathrm{MHz}, \mathrm{CDCl}_{3}\right) \delta 191.79,191.77,151.10,150.35,150.24,149.80,144.38,138.79$, 138.76, 135.24, 134.88, 134.61, 132.50, 129.56, 127.27, 127.25, 117.69, 116.68, 114.16, 114.14, $113.85,111.13,71.04,70.97,70.53,70.45,69.35,68.80,68.34,68.07,59.39,59.27,59.09,59.07$, 14.43, 14.21. HRMS (ESI): Exact mass calcd for $\mathrm{C}_{23} \mathrm{H}_{26} \mathrm{NO}_{6} \mathrm{~S}[\mathrm{M}+\mathrm{H}]^{+} 444.1481$, found 444.1470.

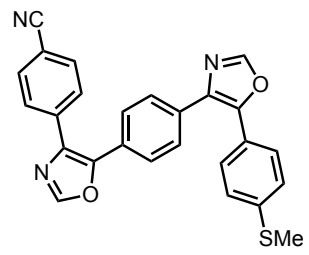

4-(5-(4-(5-(4-(methylthio)phenyl)oxazol-4-yl)phenyl)oxazol-4yl)benzonitrile (H1). Prepared according to the general van Leusen oxazole synthesis procedure using P2 (50 $\mathrm{mg}, 0.17 \mathrm{mmol}), \mathbf{M}(59 \mathrm{mg}, 0.20 \mathrm{mmol})$, $\mathrm{K}_{2} \mathrm{CO}_{3}(70 \mathrm{mg}, 0.51 \mathrm{mmol}), \mathrm{MeOH}(3 \mathrm{~mL})$ and $\mathrm{DME}(1 \mathrm{~mL})$. White solid (70 mg, 95\%). ${ }^{1} \mathrm{H}$ NMR $\left(500 \mathrm{MHz}, \mathrm{CDCl}_{3}\right) \delta 8.02(\mathrm{~s}, 1 \mathrm{H}), 7.99(\mathrm{~s}, 1 \mathrm{H}), 7.86(\mathrm{~d}, J$ $=8.2 \mathrm{~Hz}, 2 \mathrm{H}), 7.78(\mathrm{~d}, J=8.3 \mathrm{~Hz}, 2 \mathrm{H}), 7.69(\mathrm{~d}, J=8.2 \mathrm{~Hz}, 2 \mathrm{H}), 7.63(\mathrm{~d}, J=$ $8.3 \mathrm{~Hz}, 2 \mathrm{H}), 7.56(\mathrm{~d}, J=8.4 \mathrm{~Hz}, 2 \mathrm{H}), 2.55(\mathrm{~s}, 3 \mathrm{H}) .{ }^{13} \mathrm{C} \mathrm{NMR}\left(125 \mathrm{MHz}, \mathrm{CDCl}_{3}\right) \delta 150.24,149.86$, $147.14,146.35,140.55,136.66,133.47,133.33,132.46,128.21,128.19,127.52,127.33,127.30$, 
126.12, 124.75, 118.72, 111.71, 15.26. Exact mass calcd for $\mathrm{C}_{26} \mathrm{H}_{18} \mathrm{~N}_{3} \mathrm{O}_{2} \mathrm{~S}[\mathrm{M}+\mathrm{H}]^{+} 436.1120$, found 436.1120 .

\subsection{Control compound synthesis}

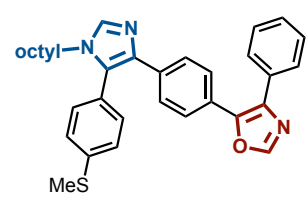

5-(4-(5-(4-(methylthio)phenyl)-1-octyl-1H-imidazol-4-yl)phenyl)-4phenyloxazole (S-PIPOP-H). Prepared according to the general van Leusen oxazole synthesis procedure using P4 (40 mg, $0.1 \mathrm{mmol}$ ), (1-Phenyl-1tosyl)methyl isocyanide (30 mg, $0.11 \mathrm{mmol}), \mathrm{K}_{2} \mathrm{CO}_{3}(40 \mathrm{mg}, 0.3 \mathrm{mmol})$, $\mathrm{MeOH}(3 \mathrm{~mL})$ and DME (1 mL). Pale white solid (20 mg, 38\%). ${ }^{1} \mathrm{H}$ NMR (500 $\left.\mathrm{MHz}, \mathrm{CDCl}_{3}\right) \delta 7.94(\mathrm{~s}, 1 \mathrm{H}), 7.68(\mathrm{~d}, J=7.8 \mathrm{~Hz}, 2 \mathrm{H}), 7.63(\mathrm{~s}, 1 \mathrm{H}), 7.53(\mathrm{~d}, J=8.5 \mathrm{~Hz}, 2 \mathrm{H}), 7.49$ $(\mathrm{d}, J=8.5 \mathrm{~Hz}, 3 \mathrm{H}), 7.40-7.33(\mathrm{~m}, 6 \mathrm{H}), 3.80(\mathrm{t}, J=7.3 \mathrm{~Hz}, 2 \mathrm{H}), 2.57(\mathrm{~s}, 3 \mathrm{H}), 1.62(\mathrm{~m}, 2 \mathrm{H}), 1.28$ $(\mathrm{m}, 2 \mathrm{H}), 1.22(\mathrm{~m}, 8 \mathrm{H}), 0.90(\mathrm{t}, J=7.0 \mathrm{~Hz}, 3 \mathrm{H}) .{ }^{13} \mathrm{C} \mathrm{NMR}\left(125 \mathrm{MHz}, \mathrm{CDCl}_{3}\right) \delta 149.56,139.90$, 137.39, 136.88, 135.17, 132.27, 131.07, 128.60, 128.56, 128.08, 127.87, 126.84, 126.61, 126.58, 126.42, 126.35, 45.22, 31.70, 30.81, 29.01, 28.91, 26.44, 22.60, 15.21, 14.08. HRMS (ESI): Exact mass calcd for $\mathrm{C}_{33} \mathrm{H}_{36} \mathrm{~N}_{3} \mathrm{OS}[\mathrm{M}+\mathrm{H}]^{+} 522.2579$, found 522.2580 .

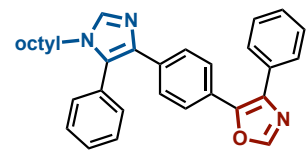

5-(4-(1-octyl-5-phenyl-1H-imidazol-4-yl)phenyl)-4-phenyloxazole

(HPIPOP-H). Prepared according to the general van Leusen oxazole synthesis procedure using P10 (40 mg, $0.11 \mathrm{mmol}$ ), (1-Phenyl-1-tosyl)methyl isocyanide (35 mg, $0.12 \mathrm{mmol}), \mathrm{K}_{2} \mathrm{CO}_{3}(50 \mathrm{mg}, 0.33 \mathrm{mmol}), \mathrm{MeOH}(3 \mathrm{~mL})$ and DME (1 mL). Pale white solid (30 mg, 57\%). ${ }^{1} \mathrm{H}$ NMR $\left(500 \mathrm{MHz}, \mathrm{CDCl}_{3}\right) \delta 7.93(\mathrm{~s}, 1 \mathrm{H}), 7.68$ $(\mathrm{d}, J=6.9 \mathrm{~Hz}, 2 \mathrm{H}), 7.64(\mathrm{~s}, 1 \mathrm{H}), 7.52-7.46(\mathrm{~m}, 7 \mathrm{H}), 7.39-7.33(\mathrm{~m}, 5 \mathrm{H}), 3.81(\mathrm{t}, J=7.3 \mathrm{~Hz}, 2 \mathrm{H})$, $1.60(\mathrm{~m}, 2 \mathrm{H}), 1.33-1.19(\mathrm{~m}, 12 \mathrm{H}), 0.88(\mathrm{t}, J=7.1 \mathrm{~Hz}, 3 \mathrm{H}) .{ }^{13} \mathrm{C} \mathrm{NMR}\left(125 \mathrm{MHz}, \mathrm{CDCl}_{3}\right) \delta 149.54$, $136.83,135.34,130.79,129.15,128.88,128.53,128.04,127.86,126.58,126.55,126.26,45.21$, $31.68,30.76,28.98,28.88,26.42,22.59,14.06$. HRMS (ESI): Exact mass calcd for $\mathrm{C}_{32} \mathrm{H}_{34} \mathrm{~N}_{3} \mathrm{O}$ $[\mathrm{M}+\mathrm{H}]^{+}$476.2702, found 476.2690.

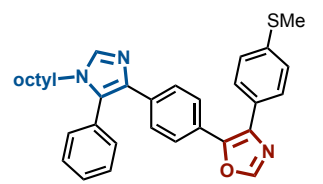

4-(4-(methylthio)phenyl)-5-(4-(1-octyl-5-phenyl-1H-imidazol-4yl)phenyl)oxazole (H-PIPOP-S). Prepared according to the general van Leusen oxazole synthesis procedure using P10 (40 mg, $0.11 \mathrm{mmol}$ ), T1 (24 $\mathrm{mg}, 0.12 \mathrm{mmol}), \mathrm{K}_{2} \mathrm{CO}_{3}(50 \mathrm{mg}, 0.33 \mathrm{mmol}), \mathrm{MeOH}(3 \mathrm{~mL})$ and DME (1 mL). ${ }^{1} \mathrm{H} \mathrm{NMR}\left(500 \mathrm{MHz}, \mathrm{CDCl}_{3}\right) \delta 7.91(\mathrm{~s}, 1 \mathrm{H}), 7.64(\mathrm{~s}, 1 \mathrm{H}), 7.60(\mathrm{~d}, J=8.3 \mathrm{~Hz}, 2 \mathrm{H}), 7.55-$ $7.43(\mathrm{~m}, 7 \mathrm{H}), 7.39-7.35(\mathrm{~m}, 2 \mathrm{H}), 7.24(\mathrm{~d}, J=8.3 \mathrm{~Hz}, 2 \mathrm{H}), 3.81(\mathrm{t}, J=7.3 \mathrm{~Hz}, 2 \mathrm{H}), 2.52(\mathrm{~s}$, $3 \mathrm{H}), 1.28(\mathrm{t}, J=7.1 \mathrm{~Hz}, 3 \mathrm{H}), 1.21(\mathrm{~s}, 8 \mathrm{H}), 0.88(\mathrm{t}, J=7.1 \mathrm{~Hz}, 3 \mathrm{H}) .{ }^{13} \mathrm{C} \mathrm{NMR}\left(125 \mathrm{MHz}, \mathrm{CDCl}_{3}\right)$ $\delta 149.55,145.88,138.44,136.84,135.36,130.79,129.17,128.96,128.92,128.16,126.58$, 126.33, 126.25, 60.41, 45.22, 31.68, 30.76, 28.98, 28.88, 26.42, 22.59, 15.61, 14.22, 14.07. Pale white solid (20 mg, 38\%). HRMS (ESI): Exact mass calcd for $\mathrm{C}_{33} \mathrm{H}_{36} \mathrm{~N}_{3} \mathrm{OS}[\mathrm{M}+\mathrm{H}]^{+} 522.2579$, found 522.2568 .

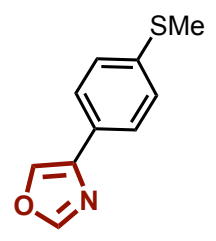

4-(4-(methylthio)phenyl)oxazole (H-OP-S). 4-Methylthiophenacyl bromide (300 $\mathrm{mg}, 1.2 \mathrm{mmol})$ was dissolved in dry formamide $(4 \mathrm{~mL})$ and heated at $150{ }^{\circ} \mathrm{C}$ for $4 \mathrm{~h}$. After cooling to room temperature, the mixture was diluted with $\mathrm{H}_{2} \mathrm{O}(50 \mathrm{~mL})$ and extracted three times with $\mathrm{CH}_{2} \mathrm{Cl}_{2}(50 \mathrm{~mL} \times 3)$. The combined organic extracts were dried $\left(\mathrm{MgSO}_{4}\right)$ and concentrated. Flash column chromatography $\left(\mathrm{SiO}_{2}, 10-40 \%\right.$ ethyl acetate in hexanes) yielded the product as a yellow solid $(50 \mathrm{mg}, 21 \%) .{ }^{1} \mathrm{H}$ NMR $\left(500 \mathrm{MHz}, \mathrm{CDCl}_{3}\right) \delta 7.95(\mathrm{~d}, J=2.1 \mathrm{~Hz}, 2 \mathrm{H}), 7.69(\mathrm{~d}, J=8.1 \mathrm{~Hz}, 2 \mathrm{H}), 7.32$ (d, $J=8.0 \mathrm{~Hz}$, 
2H), $2.53(\mathrm{~s}, 3 \mathrm{H}) .{ }^{13} \mathrm{C} \mathrm{NMR}\left(125 \mathrm{MHz}, \mathrm{CDCl}_{3}\right) \delta 151.28,140.02,138.64,133.41,127.54,126.72$, 125.97, 15.75. HRMS (ESI): Exact mass calcd for $\mathrm{C}_{10} \mathrm{H}_{10} \mathrm{NOS}[\mathrm{M}+\mathrm{H}]^{+}$192.0483, found 192.0477.

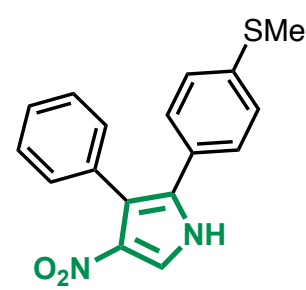

3-(4-(methylthio)phenyl)-4-nitro-2-phenyl-1 $H$-pyrrole

(H-PRP-S).

Prepared according to the general van Leusen pyrrole synthesis procedure using T1 (100 mg, $0.32 \mathrm{mmol}$ ), trans- $\beta$-Nitrostyrene (50 mg, $0.32 \mathrm{mmol}$ ), $1.6 \mathrm{M} \mathrm{n}-\mathrm{BuLi}$ in Hexane (0.24 mL, $0.38 \mathrm{mmol}), \mathrm{LiBr}(170 \mathrm{mg}, 2.0 \mathrm{mmol})$ and THF (10 mL). Yellow solid (60 mg, 62\%). ${ }^{1} \mathrm{H}$ NMR (500 MHz, $\left.\mathrm{CDCl}_{3}\right) \delta 8.66$ $(\mathrm{s}, 1 \mathrm{H}), 7.83(\mathrm{~d}, J=3.5 \mathrm{~Hz}, 1 \mathrm{H}), 7.37(\mathrm{dt}, J=4.5,2.4 \mathrm{~Hz}, 3 \mathrm{H}), 7.33-7.29(\mathrm{~m}$, $2 \mathrm{H}), 7.14(\mathrm{~d}, J=8.5 \mathrm{~Hz}, 2 \mathrm{H}), 7.06(\mathrm{~d}, J=8.5 \mathrm{~Hz}, 2 \mathrm{H}), 2.47(\mathrm{~s}, 3 \mathrm{H}) .{ }^{13} \mathrm{C} \mathrm{NMR}\left(125 \mathrm{MHz}, \mathrm{CDCl}_{3}\right)$ $\delta 138.86,136.59,133.96,132.00,130.73,130.02,128.22,127.54,127.49,127.27,126.29,120.11$, 117.48, 15.32. HRMS (ESI): Exact mass calcd for $\mathrm{C}_{17} \mathrm{H}_{15} \mathrm{~N}_{2} \mathrm{O}_{2} \mathrm{~S}[\mathrm{M}+\mathrm{H}]^{+} 311.0854$, found 311.0853 .

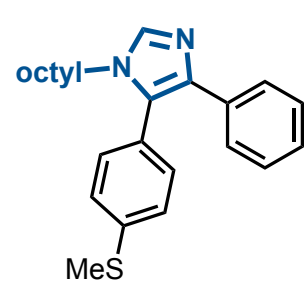

\section{5-(4-(methylthio)phenyl)-1-octyl-4-phenyl-1H-imidazole}

(S-PIP-H). Prepared according to the general van Leusen imidazole synthesis procedure using 4-(Methylthio)benzaldehyde (0.13 mL, $1 \mathrm{mmol})$, TosMIC (327 mg, 1.2 mmol), octylamine (0.2 mL, $1.2 \mathrm{mmol}), \mathrm{K}_{2} \mathrm{CO}_{3}(419 \mathrm{mg}, 3 \mathrm{mmol}), \mathrm{MeOH}(6$ $\mathrm{mL})$ and DME (2 mL). Colorless liquid (200 mg, 53\%). ${ }^{1} \mathrm{H}$ NMR (500 MHz, $\left.\mathrm{CDCl}_{3}\right) \delta 7.62(\mathrm{~s}, 1 \mathrm{H}), 7.51(\mathrm{~d}, J=7.2 \mathrm{~Hz}, 2 \mathrm{H}), 7.33(\mathrm{~d}, J=8.3 \mathrm{~Hz}, 2 \mathrm{H}), 7.24$ $(\mathrm{dd}, J=20.1,8.1 \mathrm{~Hz}, 4 \mathrm{H}), 7.16(\mathrm{t}, J=7.3 \mathrm{~Hz}, 1 \mathrm{H}), 3.82-3.75(\mathrm{~m}, 2 \mathrm{H}), 2.57(\mathrm{~s}, 3 \mathrm{H}), 1.60(\mathrm{t}, J=$ $7.3 \mathrm{~Hz}, 2 \mathrm{H}), 1.31-1.18(\mathrm{~m}, 10 \mathrm{H}), 0.89$ (t, $J=7.1 \mathrm{~Hz}, 3 \mathrm{H}) .{ }^{13} \mathrm{C}$ NMR $\left(125 \mathrm{MHz}, \mathrm{CDCl}_{3}\right) \delta 139.42$, $138.19,136.66,134.67,131.15,128.13,127.89,127.28,126.52,126.36,126.22,45.14,31.70$, $30.83,29.02,28.92,26.46,22.60,15.26,14.08$.

\subsection{Sequence-defined oligomers synthesis}

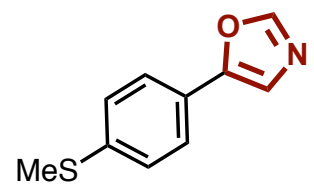

5-(4-(methylthio)phenyl)oxazole (PO). Prepared according to the general van Leusen imidazole synthesis procedure using 4-(Methylthio)benzaldehyde (0.13 mL, $1.0 \mathrm{mmol})$, TosMIC (215 mg, $1.1 \mathrm{mmol}), \mathrm{K}_{2} \mathrm{CO}_{3}(414 \mathrm{mg}, 3.0$ $\mathrm{mmol}), \mathrm{MeOH}(3 \mathrm{~mL})$ and DME (1 mL). White solid (173 mg, 91\%). ${ }^{1} \mathrm{H}$ NMR $\left(500 \mathrm{MHz}, \mathrm{CDCl}_{3}\right) \delta 7.92(\mathrm{~s}, 1 \mathrm{H}), 7.60(\mathrm{~d}, J=8.4 \mathrm{~Hz}, 2 \mathrm{H}), 7.35-7.30(\mathrm{~m}, 3 \mathrm{H}), 2.54(\mathrm{~s}, 3 \mathrm{H}) .{ }^{13} \mathrm{C}$ NMR (125 MHz, $\left.\mathrm{CDCl}_{3}\right) \delta 180.01,151.26,150.22,139.54,126.54,124.76,124.46,121.10,15.53$. HRMS (ESI): Exact mass calcd for $[\mathrm{M}+\mathrm{H}]^{+} \mathrm{C}_{10} \mathrm{H}_{10} \mathrm{NOS} 192.0483$, found 192.0474.

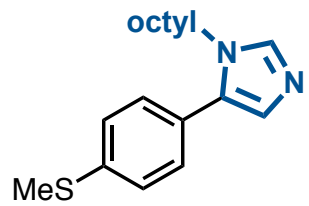

5-(4-(methylthio)phenyl)-1-octyl-1H-imidazole (PI). Prepared according to the general van Leusen imidazole synthesis procedure using 4(Methylthio)benzaldehyde $(0.07 \mathrm{~mL}, 0.5 \mathrm{mmol})$, TosMIC (100 mg, 0.51 mmol), octylamine (0.4 mL, $2.1 \mathrm{mmol}), \mathrm{K}_{2} \mathrm{CO}_{3}(210 \mathrm{mg}, 3.0 \mathrm{mmol}), \mathrm{MeOH}(3$ $\mathrm{mL})$ and DME (1 mL). Brown oil (100 mg, 66\%). ${ }^{1} \mathrm{H}$ NMR (500 MHz, $\left.\mathrm{CDCl}_{3}\right)$ $\delta 7.56(\mathrm{~s}, 1 \mathrm{H}), 7.34-7.29(\mathrm{~m}, 4 \mathrm{H}), 7.06(\mathrm{~s}, 1 \mathrm{H}), 4.00-3.91(\mathrm{~m}, 2 \mathrm{H}), 2.55(\mathrm{~s}, 3 \mathrm{H}), 1.25(\mathrm{~m}, J=$ $33.7 \mathrm{~Hz}, 12 \mathrm{H}), 0.89(\mathrm{t}, J=7.1 \mathrm{~Hz}, 3 \mathrm{H}) .{ }^{13} \mathrm{C} \mathrm{NMR}\left(125 \mathrm{MHz}, \mathrm{CDCl}_{3}\right) \delta 138.61,138.09,132.41$, $129.12,128.19,126.84,126.48,45.33,38.22$, 31.77, 31.69, 30.87, 29.56, 29.20, 29.03, 28.92, 26.85, 26.45, 22.60, 15.61, 14.09, 14.07. HRMS (ESI): Exact mass calcd for $[\mathrm{M}+\mathrm{H}]^{+} \mathrm{C}_{18} \mathrm{H}_{27} \mathrm{~N}_{2} \mathrm{~S}$ 303.1895 , found 303.1884 . 


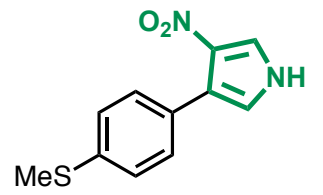

3-(4-(methylthio)phenyl)-4-nitro-1H-pyrrole (PR). Prepared according to the general van Leusen pyrrole synthesis procedure using 4-(Methylthio)- $\beta$ nitrostyrene (40 mg, $0.2 \mathrm{mmol}$ ), TosMIC (43 mg, $0.22 \mathrm{mmol}), 1.6 \mathrm{M} \mathrm{n}-\mathrm{BuLi}$ in Hexane (0.14 mL, $0.22 \mathrm{mmol}), \mathrm{LiBr}(155 \mathrm{mg}, 1.2 \mathrm{mmol})$ and THF $(3 \mathrm{~mL})$. Yellow solid (14 mg, 30\%). ${ }^{1} \mathrm{H}$ NMR $\left(500 \mathrm{MHz}, \mathrm{CDCl}_{3}\right) \delta 8.54(\mathrm{~s}, 1 \mathrm{H}), 7.68(\mathrm{~s}, 1 \mathrm{H}), 7.30(\mathrm{~d}, J=$ $8.3 \mathrm{~Hz}, 2 \mathrm{H}), 7.21(\mathrm{~d}, J=8.3 \mathrm{~Hz}, 2 \mathrm{H}), 6.67(\mathrm{~s}, 1 \mathrm{H}), 2.45(\mathrm{~s}, 3 \mathrm{H}) .{ }^{13} \mathrm{C}$ NMR $\delta 137.91,129.77,128.69$, 126.19, 121.26, 118.15, 15.82. HRMS (ESI): Exact mass calcd for $[\mathrm{M}+\mathrm{H}]^{+} \mathrm{C}_{11} \mathrm{H}_{11} \mathrm{~N}_{2} \mathrm{O}_{2} \mathrm{~S}$ 235.0541 , found 235.0534 .

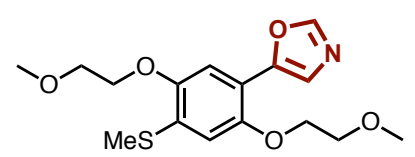

5-(2,5-bis(2-methoxyethoxy)-4-(methylthio)phenyl)oxazole (GO). Prepared according to the general van Leusen imidazole synthesis procedure using 2,5-bis(2-methoxyethoxy)-4-(methylthio)benzaldehyde (30 mg, $0.1 \mathrm{mmol}$ ), TosMIC (24 mg, $0.12 \mathrm{mmol}), \mathrm{K}_{2} \mathrm{CO}_{3}(42 \mathrm{mg}, 0.3$ $\mathrm{mmol}), \mathrm{MeOH}(3 \mathrm{~mL})$ and DME (1 mL). White solid $(28 \mathrm{mg}, 82 \%) .{ }^{1} \mathrm{H} \mathrm{NMR}\left(500 \mathrm{MHz}, \mathrm{CDCl}_{3}\right)$ $\delta 7.90(\mathrm{~s}, 1 \mathrm{H}), 7.65(\mathrm{~s}, 1 \mathrm{H}), 7.30(\mathrm{~s}, 1 \mathrm{H}), 6.82(\mathrm{~s}, 1 \mathrm{H}), 4.24(\mathrm{dt}, J=9.6,4.8 \mathrm{~Hz}, 4 \mathrm{H}), 3.84(\mathrm{dt}, J=$ 9.7, $4.7 \mathrm{~Hz}, 4 \mathrm{H}), 3.51(\mathrm{~d}, J=1.7 \mathrm{~Hz}, 6 \mathrm{H}), 2.48(\mathrm{~s}, 3 \mathrm{H}) .{ }^{13} \mathrm{C} \mathrm{NMR}\left(125 \mathrm{MHz}, \mathrm{CDCl}_{3}\right) \delta 150.12$, 149.85, 149.13, 129.35, 125.42, 114.47, 110.88, 109.89, 71.11, 71.07, 69.32, 68.45, 59.39, 59.24, 14.83. HRMS (ESI): Exact mass calcd for $\mathrm{C}_{16} \mathrm{H}_{22} \mathrm{NO}_{5} \mathrm{~S}[\mathrm{M}+\mathrm{H}]^{+} 340.1219$, found 340.1210.

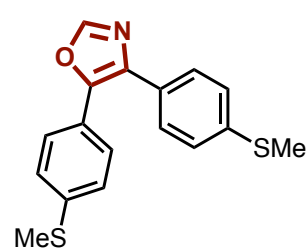

4,5-bis(4-(methylthio)phenyl)oxazole (POP). Prepared according to the general van Leusen imidazole synthesis procedure using 4(Methylthio)benzaldehyde (0.04 mL, $0.3 \mathrm{mmol})$, T1 (105 mg, $0.33 \mathrm{mmol}$ ), $\mathrm{K}_{2} \mathrm{CO}_{3}(124 \mathrm{mg}, 0.9 \mathrm{mmol}), \mathrm{MeOH}(3 \mathrm{~mL})$ and DME $(1 \mathrm{~mL}) .{ }^{1} \mathrm{H} \mathrm{NMR}(500$ $\left.\mathrm{MHz} \mathrm{CDCl}_{3}\right) \delta 7.95(\mathrm{~s}, 1 \mathrm{H}), 7.60(\mathrm{~d}, J=8.4 \mathrm{~Hz}, 2 \mathrm{H}), 7.54(\mathrm{~d}, J=8.5 \mathrm{~Hz}, 2 \mathrm{H})$, $7.28(\mathrm{~d}, J=7.4 \mathrm{~Hz}, 4 \mathrm{H}), 2.54(\mathrm{~d}, J=1.1 \mathrm{~Hz}, 6 \mathrm{H}) .{ }^{13} \mathrm{C} \mathrm{NMR}\left(125 \mathrm{MHz}, \mathrm{CDCl}_{3}\right)$ $\delta 149.62,145.35,139.83,138.71,134.04,128.79,128.17,127.05,126.35,126.15,125.18,15.56$, 15.34. HRMS (ESI): Exact mass calcd for $\mathrm{C}_{17} \mathrm{H}_{16} \mathrm{NOS}_{2}[\mathrm{M}+\mathrm{H}]^{+} 314.0673$, found 314.0663.

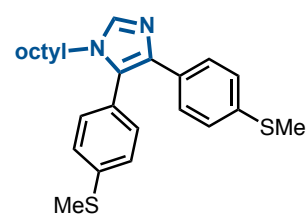

4,5-bis(4-(methylthio)phenyl)-1-nonyl-1H-imidazole (PIP). Prepared according to the general van Leusen imidazole synthesis procedure using 4(Methylthio)benzaldehyde (0.03 mL, $0.2 \mathrm{mmol}), \mathbf{T 1}(50 \mathrm{mg}, 0.15 \mathrm{mmol})$, octylamine (0.04 mL, $0.3 \mathrm{mmol}), \mathrm{K}_{2} \mathrm{CO}_{3}(65 \mathrm{mg}, 0.47 \mathrm{mmol}), \mathrm{MeOH}(6 \mathrm{~mL})$ and DME (2 mL). Yellow oil (30 mg, 35\%). ${ }^{1} \mathrm{H}$ NMR $\left(500 \mathrm{MHz}, \mathrm{CDCl}_{3}\right) \delta$ $7.58(\mathrm{~s}, 1 \mathrm{H}), 7.40(\mathrm{~d}, J=8.5 \mathrm{~Hz}, 2 \mathrm{H}), 7.31(\mathrm{~d}, J=8.3 \mathrm{~Hz}, 2 \mathrm{H}), 7.23(\mathrm{~d}, J=8.3 \mathrm{~Hz}, 2 \mathrm{H}), 7.11(\mathrm{~d}$, $J=8.5 \mathrm{~Hz}, 2 \mathrm{H}), 3.76(\mathrm{t}, J=7.4 \mathrm{~Hz}, 2 \mathrm{H}), 2.55(\mathrm{~s}, 3 \mathrm{H}), 2.44(\mathrm{~s}, 3 \mathrm{H}), 1.57(\mathrm{~m}, 4 \mathrm{H}), 1.19(\mathrm{~m}, 8 \mathrm{H})$, $0.87(\mathrm{~m}, J=7.0 \mathrm{~Hz}, 3 \mathrm{H}) .{ }^{13} \mathrm{C} \mathrm{NMR}\left(125 \mathrm{MHz}, \mathrm{CDCl}_{3}\right) \delta 139.53,137.74,136.65,135.88,131.74$, $131.12,127.73,127.16,126.89,126.49,126.37,105.68,45.16,31.70,30.83,29.71,29.01,28.92$, 26.46, 22.60, 15.92, 15.25, 14.08. HRMS (ESI): Exact mass calcd for $\mathrm{C}_{25} \mathrm{H}_{32} \mathrm{~N}_{2} \mathrm{~S}_{2}[\mathrm{M}+\mathrm{H}]^{+}$ 425.2085, found 425.2077 .

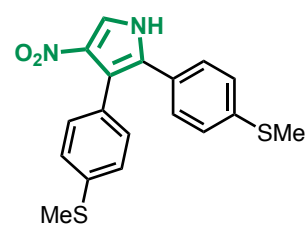

2,3-bis(4-(methylthio)phenyl)-4-nitro-1H-pyrrole (PRP). Prepared according to the general van Leusen pyrrole synthesis procedure using 4(Methylthio)- $\beta$-nitrostyrene (78 mg, $0.4 \mathrm{mmol})$, T1 (130 $\mathrm{mg}, 0.44 \mathrm{mmol}$ ), 1.6M n-BuLi in Hexane (0.275 mL, $0.44 \mathrm{mmol}), \mathrm{LiBr}(208 \mathrm{mg}, 2.4 \mathrm{mmol})$ and THF $(10 \mathrm{~mL})$. Yellow solid (100 mg, 75\%). ${ }^{1} \mathrm{H}$ NMR $\left(500 \mathrm{MHz}, \mathrm{CDCl}_{3}\right) \delta$ $8.67(\mathrm{~s}, 1 \mathrm{H}), 7.82(\mathrm{~d}, J=3.5 \mathrm{~Hz}, 1 \mathrm{H}), 7.27-7.19(\mathrm{~m}, 4 \mathrm{H}), 7.15(\mathrm{~d}, J=8.4 \mathrm{~Hz}, 2 \mathrm{H}), 7.07(\mathrm{~d}, J=$ 
$8.4 \mathrm{~Hz}, 2 \mathrm{H}), 2.53(\mathrm{~s}, 3 \mathrm{H}), 2.48(\mathrm{~s}, 3 \mathrm{H}) .{ }^{13} \mathrm{C} \mathrm{NMR}\left(125 \mathrm{MHz}, \mathrm{CDCl}_{3}\right) \delta 138.96,137.80,136.50$, $131.15,130.11,128.47,127.56,127.18,126.32,126.00,120.27,116.84,15.51,15.31$. HRMS (ESI): Exact mass calcd for $\mathrm{C}_{18} \mathrm{H}_{17} \mathrm{~N}_{2} \mathrm{O}_{2} \mathrm{~S}_{2}[\mathrm{M}+\mathrm{H}]^{+} 357.0731$, found 357.0719.

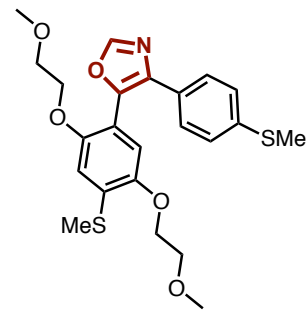

5-(2,5-bis(2-methoxyethoxy)-4-(methylthio)phenyl)-4-(4(methylthio)phenyl)oxazole (GOP). Prepared according to the general van Leusen imidazole synthesis procedure using 2,5-bis(2-methoxyethoxy)-4(methylthio)benzaldehyde (75 mg, $0.25 \mathrm{mmol}$ ), T1 (87 mg, $0.28 \mathrm{mmol})$, $\mathrm{K}_{2} \mathrm{CO}_{3}(104 \mathrm{mg}, 0.75 \mathrm{mmol}), \mathrm{MeOH}(3 \mathrm{~mL})$ and DME $(1 \mathrm{~mL})$. Yellow oil $(95$ $\mathrm{mg}, 82 \%) .{ }^{1} \mathrm{H} \mathrm{NMR}\left(500 \mathrm{MHz}, \mathrm{CDCl}_{3}\right) \delta 7.97(\mathrm{~s}, 1 \mathrm{H}), 7.52(\mathrm{~d}, J=8.6 \mathrm{~Hz}, 2 \mathrm{H})$, $7.22(\mathrm{~d}, J=8.6 \mathrm{~Hz}, 2 \mathrm{H}), 6.97(\mathrm{~s}, 1 \mathrm{H}), 6.83(\mathrm{~s}, 1 \mathrm{H}), 4.15-4.12(\mathrm{~m}, 2 \mathrm{H}), 3.93$ $(\mathrm{t}, J=5.0 \mathrm{~Hz}, 2 \mathrm{H}), 3.80-3.77(\mathrm{~m}, 2 \mathrm{H}), 3.48(\mathrm{~s}, 3 \mathrm{H}), 3.23(\mathrm{~s}, 3 \mathrm{H}), 3.21-3.18(\mathrm{~m}, 2 \mathrm{H}), 2.50(\mathrm{~d}, J$ $=4.9 \mathrm{~Hz}, 6 \mathrm{H}) .{ }^{13} \mathrm{C}$ NMR $\left(125 \mathrm{MHz}, \mathrm{CDCl}_{3}\right) \delta 151.17,149.99,149.80,142.39,137.90,135.22$, 131.56, 129.44, 127.22, 126.08, 114.97, 114.01, 111.57, 70.97, 70.59, 69.27, 69.00, 59.37, 59.12, 15.81, 15.78, 14.49. HRMS (ESI): Exact mass calcd for $\mathrm{C}_{23} \mathrm{H}_{27} \mathrm{NO}_{5} \mathrm{~S}_{2}[\mathrm{M}+\mathrm{H}]^{+} 462.1392$, found 462.1409 .

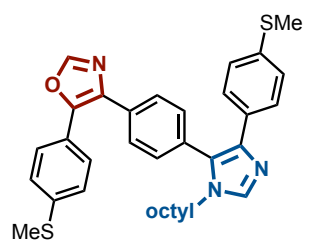

5-(4-(methylthio)phenyl)-4-(4-(4-(4-(methylthio)phenyl)-1-octyl-1Himidazol-5-yl)phenyl)oxazole (POPIP). Prepared according to the general van Leusen imidazole procedure using P2 (35 mg, $0.12 \mathrm{mmol})$, T1 (45 mg, $0.144 \mathrm{mmol})$, octylamine ( $36 \mathrm{mg}, 0.30 \mathrm{mmol}), \mathrm{K}_{2} \mathrm{CO}_{3}(50 \mathrm{mg}, 0.36 \mathrm{mmol})$, $\mathrm{MeOH}(6 \mathrm{~mL})$ and DME (2 mL). Light yellow solid (60 mg, 90\%). ${ }^{1} \mathrm{H}$ NMR $\left(500 \mathrm{MHz}, \mathrm{CDCl}_{3}\right) \delta 8.01(\mathrm{~s}, 1 \mathrm{H}), 7.80(\mathrm{~d}, J=8.1 \mathrm{~Hz}, 2 \mathrm{H}), 7.63(\mathrm{~s}, 1 \mathrm{H}), 7.58$ $(\mathrm{d}, J=8.5 \mathrm{~Hz}, 2 \mathrm{H}), 7.45(\mathrm{~d}, J=8.5 \mathrm{~Hz}, 2 \mathrm{H}), 7.37(\mathrm{~d}, J=8.1 \mathrm{~Hz}, 2 \mathrm{H}), 7.14(\mathrm{~d}, J=8.5 \mathrm{~Hz}, 2 \mathrm{H})$, $3.84(\mathrm{t}, J=7.4 \mathrm{~Hz}, 2 \mathrm{H}), 2.55(\mathrm{~s}, 3 \mathrm{H}), 2.48(\mathrm{~s}, 3 \mathrm{H}), 1.66-1.60(\mathrm{~m}, 2 \mathrm{H}), 1.23(\mathrm{~s}, 10 \mathrm{H}), 0.87(\mathrm{t}, J=$ $6.9 \mathrm{~Hz}, 3 \mathrm{H}) .{ }^{13} \mathrm{C} \mathrm{NMR}\left(125 \mathrm{MHz}, \mathrm{CDCl}_{3}\right) \delta 149.80,146.10,140.38,138.01,136.85,136.02$, $132.46,131.76,131.06,130.77,128.33,127.82,127.21,127.07,126.50,126.14,124.93,45.33$, 31.70, 30.89, 29.06, 28.97, 26.53, 22.60, 15.96, 15.28, 14.06. HRMS (ESI): Exact mass calcd for $\mathrm{C}_{34} \mathrm{H}_{38} \mathrm{~N}_{3} \mathrm{OS}_{2}[\mathrm{M}+\mathrm{H}]^{+}$568.2456, found 568.2463.

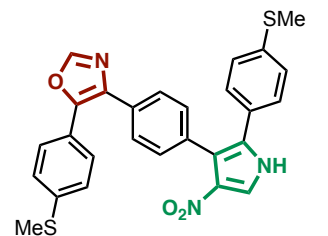

5-(4-(methylthio)phenyl)-4-(4-(2-(4-(methylthio)phenyl)-4-nitro-1Hpyrrol-3-yl)phenyl)oxazole (POPRP). Prepared according to the general van Leusen pyrrole synthesis procedure using P2 (70 mg, $0.21 \mathrm{mmol})$, T1 (50 mg, $0.25 \mathrm{mmol}), 1.6 \mathrm{M} \mathrm{n}-\mathrm{BuLi}$ in Hexane $(0.16 \mathrm{~mL}, 0.25 \mathrm{mmol}), \mathrm{LiBr}(110 \mathrm{mg}$, $1.26 \mathrm{mmol})$ and THF (10 mL). Brown solid (70 mg, 67\%). ${ }^{1} \mathrm{H}$ NMR $(500 \mathrm{MHz}$, $\left.\mathrm{CDCl}_{3}\right) \delta 8.75(\mathrm{~s}, 1 \mathrm{H}), 7.97(\mathrm{~s}, 1 \mathrm{H}), 7.85(\mathrm{~d}, J=3.5 \mathrm{~Hz}, 1 \mathrm{H}), 7.67(\mathrm{~d}, J=8.4 \mathrm{~Hz}, 2 \mathrm{H}), 7.58(\mathrm{~d}, J=$ $8.6 \mathrm{~Hz}, 2 \mathrm{H}), 7.33(\mathrm{~d}, J=8.3 \mathrm{~Hz}, 2 \mathrm{H}), 7.27(\mathrm{~d}, J=8.6 \mathrm{~Hz}, 2 \mathrm{H}), 7.15(\mathrm{~d}, J=8.6 \mathrm{~Hz}, 2 \mathrm{H}), 7.09$ (d, $J$ $=8.6 \mathrm{~Hz}, 2 \mathrm{H}), 2.53(\mathrm{~s}, 3 \mathrm{H}), 2.47(\mathrm{~s}, 3 \mathrm{H}) .{ }^{13} \mathrm{C} \mathrm{NMR}\left(125 \mathrm{MHz}, \mathrm{CDCl}_{3}\right) \delta 149.64,139.95,139.08$, 136.54, 134.21, 131.99, 131.21, 131.13, 130.31, 127.67, 127.57, 127.14, 126.34, 126.14, 125.12, 120.25, 117.03, 15.33. HRMS (ESI): Exact mass calcd for $\mathrm{C}_{27} \mathrm{H}_{22} \mathrm{~N}_{3} \mathrm{O}_{3} \mathrm{~S}_{2}[\mathrm{M}+\mathrm{H}]^{+} 500.1103$, found 500.1101 . 


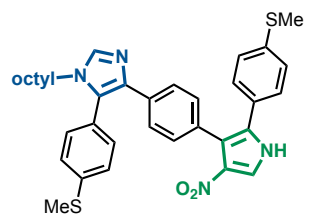

5-(4-(methylthio)phenyl)-4-(4-(2-(4-(methylthio)phenyl)-4-nitro-1Hpyrrol-3-yl)phenyl)-1-octyl-1H-imidazole (PIPRP). Prepared according to the general van Leusen pyrrole synthesis procedure using P4 (50 mg, 0.11 mmol), T1 (24 mg, $0.12 \mathrm{mmol}), 1.6 \mathrm{M} \mathrm{n}-\mathrm{BuLi}$ in Hexane $(0.075 \mathrm{~mL}, 0.12$ $\mathrm{mmol}), \mathrm{LiBr}(57 \mathrm{mg}, 0.66 \mathrm{mmol})$ and THF $(10 \mathrm{~mL})$. Yellow solid $(50 \mathrm{mg}$, 75\%). ${ }^{1} \mathrm{H}$ NMR $\left(500 \mathrm{MHz}, \mathrm{CDCl}_{3}\right) \delta 8.79(\mathrm{~s}, 1 \mathrm{H}), 7.70(\mathrm{~d}, J=3.5 \mathrm{~Hz}, 1 \mathrm{H}), 7.52(\mathrm{~s}, 1 \mathrm{H}), 7.41(\mathrm{~d}$, $J=8.4 \mathrm{~Hz}, 2 \mathrm{H}), 7.24(\mathrm{~d}, J=8.4 \mathrm{~Hz}, 2 \mathrm{H}), 7.21(\mathrm{~s}, 2 \mathrm{H}), 7.06(\mathrm{~d}, J=8.4 \mathrm{~Hz}, 2 \mathrm{H}), 7.00(\mathrm{~d}, J=8.6$ $\mathrm{Hz}, 2 \mathrm{H}), 6.95(\mathrm{~d}, J=8.6 \mathrm{~Hz}, 2 \mathrm{H}), 3.69(\mathrm{t}, J=7.3 \mathrm{~Hz}, 2 \mathrm{H}), 2.47(\mathrm{~s}, 3 \mathrm{H}), 2.35(\mathrm{~s}, 3 \mathrm{H}), 1.50(\mathrm{~s}, 3 \mathrm{H})$, $1.20(\mathrm{~d}, J=7.0 \mathrm{~Hz}, 2 \mathrm{H}), 1.12(\mathrm{~s}, 8 \mathrm{H}), 0.80(\mathrm{t}, J=7.1 \mathrm{~Hz}, 3 \mathrm{H}) .{ }^{13} \mathrm{C} \mathrm{NMR}\left(125 \mathrm{MHz}, \mathrm{CDCl}_{3}\right) \delta$ 138.61, 137.84, 136.69, 136.52, 133.77, 131.19, 130.62, 128.16, 127.54, 127.41, 127.14, 126.42, 126.34, 126.11, 120.15, 45.16, 31.72, 30.82, 29.03, 28.93, 26.45, 22.61, 15.37, 15.25, 14.09. HRMS (ESI): Exact mass calcd for $\mathrm{C}_{35} \mathrm{H}_{39} \mathrm{~N}_{4} \mathrm{O}_{2} \mathrm{~S}_{2}[\mathrm{M}+\mathrm{H}]^{+} 611.2514$, found 611.2529.

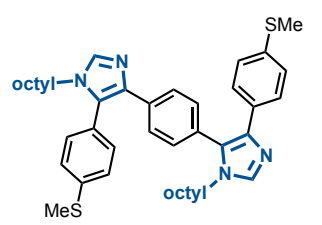

4-(4-(methylthio)phenyl)-5-(4-(5-(4-(methylthio)phenyl)-1-octyl-1Himidazol-4-yl)phenyl)-1-octyl-1H-imidazole (PIPIP). Prepared according to the general van Leusen imidazole procedure using P4 (54 mg, $0.13 \mathrm{mmol}), \mathbf{T 1}$ (50 mg, $0.16 \mathrm{mmol})$, octylamine $(20 \mathrm{mg}, 0.16 \mathrm{mmol}), \mathrm{K}_{2} \mathrm{CO}_{3}(54 \mathrm{mg}, 0.39$ $\mathrm{mmol}), \mathrm{MeOH}(6 \mathrm{~mL})$ and DME (2 mL). Light yellow solid $(42 \mathrm{mg}, 48 \%) .{ }^{1} \mathrm{H}$ NMR (500 MHz, $\left.\mathrm{CDCl}_{3}\right) \delta 7.65(\mathrm{~s}, 1 \mathrm{H}), 7.63(\mathrm{~d}, J=8.5 \mathrm{~Hz}, 2 \mathrm{H}), 7.58(\mathrm{~s}, 1 \mathrm{H}), 7.43(\mathrm{~d}, J=8.6 \mathrm{~Hz}$, 2H), $7.36(\mathrm{~d}, J=8.5 \mathrm{~Hz}, 2 \mathrm{H}), 7.31(\mathrm{~d}, J=8.5 \mathrm{~Hz}, 2 \mathrm{H}), 7.18(\mathrm{~d}, J=8.5 \mathrm{~Hz}, 2 \mathrm{H}), 7.10(\mathrm{~d}, J=8.6$ $\mathrm{Hz}, 2 \mathrm{H}), 3.81(\mathrm{t}, J=7.3 \mathrm{~Hz}, 2 \mathrm{H}), 3.77(\mathrm{t}, J=7.3 \mathrm{~Hz}, 2 \mathrm{H}), 2.57(\mathrm{~s}, 3 \mathrm{H}), 2.46(\mathrm{~s}, 3 \mathrm{H}), 1.59(\mathrm{~m}, J=$ $22.8,6.6 \mathrm{~Hz}, 4 \mathrm{H}), 1.32-1.18(\mathrm{~m}, 21 \mathrm{H}), 0.88(\mathrm{~m}, J=14.1,7.1 \mathrm{~Hz}, 6 \mathrm{H}) .{ }^{13} \mathrm{C} \mathrm{NMR}(125 \mathrm{MHz}$, $\left.\mathrm{CDCl}_{3}\right) \delta 136.88,136.51,131.10,130.62,128.54,128.47,128.41,126.98,126.88,126.72,126.54$, 126.41, 45.19, 31.72, 31.69, 30.83, 30.81, 29.03, 28.93, 26.48, 26.45, 22.61, 22.60, 16.03, 15.20, 14.08. HRMS (ESI): Exact mass calcd for $\mathrm{C}_{42} \mathrm{H}_{55} \mathrm{~N}_{4} \mathrm{~S}_{2}[\mathrm{M}+\mathrm{H}]^{+} 679.3860$, found 679.3868 .

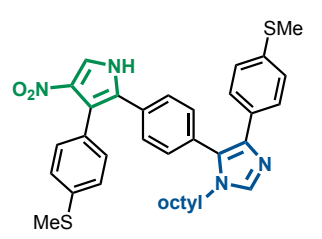

4-(4-(methylthio)phenyl)-5-(4-(3-(4-(methylthio)phenyl)-4-nitro-1Hpyrrol-2-yl)phenyl)-1-octyl-1H-imidazole (PRPIP). Prepared according to the general van Leusen imidazole procedure using P6 (17 mg, $0.05 \mathrm{mmol}), \mathbf{T 1}$ (19 mg, $0.06 \mathrm{mmol})$, octylamine (7.7 mg, $0.06 \mathrm{mmol}), \mathrm{K}_{2} \mathrm{CO}_{3}(21 \mathrm{mg}, 0.15$ $\mathrm{mmol}), \mathrm{MeOH}(3 \mathrm{~mL})$ and DME (1 mL). Yellow solid (42 mg, 48\%). ${ }^{1} \mathrm{H} \mathrm{NMR}$ $\left(500 \mathrm{MHz}, \mathrm{CDCl}_{3}\right) \delta 9.00(\mathrm{~s}, 1 \mathrm{H}), 7.88(\mathrm{~d}, J=3.5 \mathrm{~Hz}, 1 \mathrm{H}), 7.60(\mathrm{~s}, 1 \mathrm{H}), 7.35(\mathrm{~d}, J=8.5 \mathrm{~Hz}, 2 \mathrm{H})$, $7.26(\mathrm{~d}, J=3.3 \mathrm{~Hz}, 8 \mathrm{H}), 7.11(\mathrm{~d}, J=8.5 \mathrm{~Hz}, 2 \mathrm{H}), 3.79(\mathrm{t}, J=7.4 \mathrm{~Hz}, 2 \mathrm{H}), 2.54(\mathrm{~s}, 3 \mathrm{H}), 2.47(\mathrm{~s}$, $3 \mathrm{H}), 1.58(\mathrm{~m}, 2 \mathrm{H}), 1.28(\mathrm{~m}, 2 \mathrm{H}), 1.21(\mathrm{~m}, 8 \mathrm{H}), 0.88(\mathrm{t}, J=7.1 \mathrm{~Hz}, 3 \mathrm{H}) .{ }^{13} \mathrm{C} \mathrm{NMR}(125 \mathrm{MHz}$, $\left.\mathrm{CDCl}_{3}\right) \delta 138.20,136.99,136.29,131.16,131.09,130.95,130.49,129.63,128.32,127.68,127.11$, 126.43, 126.01, 120.63, 117.72, 45.34, 31.70, 30.78, 29.04, 28.93, 26.48, 22.61, 15.90, 15.51, 14.08. HRMS (ESI): Exact mass calcd for $\mathrm{C}_{35} \mathrm{H}_{39} \mathrm{~N}_{4} \mathrm{O}_{2} \mathrm{~S}_{2}[\mathrm{M}+\mathrm{H}]^{+} 611.2514$, found 611.2510.

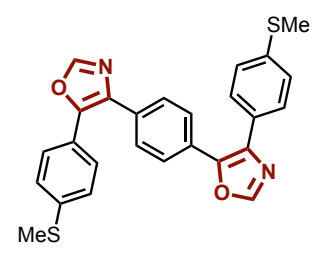

4-(4-(methylthio)phenyl)-5-(4-(5-(4-(methylthio)phenyl)oxazol-4yl)phenyl)oxazole (POPOP). Prepared according to the general van Leusen oxazole synthesis procedure using P2 (30 mg, $0.10 \mathrm{mmol}$ ), T1 (38 mg, 0.12 $\mathrm{mmol}), \mathrm{K}_{2} \mathrm{CO}_{3}(41 \mathrm{mg}, 0.30 \mathrm{mmol}), \mathrm{MeOH}(3 \mathrm{~mL})$ and DME $(1 \mathrm{~mL})$. Light yellow solid (25 mg, 56\%). ${ }^{1} \mathrm{H}$ NMR $\left(500 \mathrm{MHz}, \mathrm{CDCl}_{3}\right) \delta 7.98(\mathrm{~d}, J=2.0 \mathrm{~Hz}$, $2 \mathrm{H}), 7.72(\mathrm{~d}, J=8.6 \mathrm{~Hz}, 2 \mathrm{H}), 7.65(\mathrm{dd}, J=13.4,8.6 \mathrm{~Hz}, 4 \mathrm{H}), 7.56(\mathrm{~d}, J=8.6$ $\mathrm{Hz}, 2 \mathrm{H}), 7.27$ (d, $J=3.1 \mathrm{~Hz}, 2 \mathrm{H}), 2.54(\mathrm{~s}, 6 \mathrm{H}) .{ }^{13} \mathrm{C} \mathrm{NMR}\left(125 \mathrm{MHz}, \mathrm{CDCl}_{3}\right) \delta 149.85,149.79$, 145.29, 140.31, 128.68, 128.31, 128.02, 127.26, 126.81, 126.36, 126.15, 124.92, 15.55, 15.30 . HRMS (ESI): Exact mass calcd for $\mathrm{C}_{26} \mathrm{H}_{21} \mathrm{~N}_{2} \mathrm{O}_{2} \mathrm{~S}_{2}[\mathrm{M}+\mathrm{H}]^{+} 457.1044$, found 457.1029. 


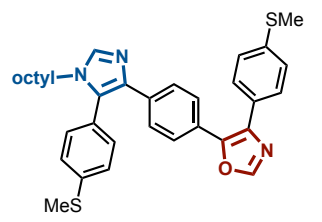

4-(4-(methylthio)phenyl)-5-(4-(5-(4-(methylthio)phenyl)-1-octyl-1 Himidazol-4-yl)phenyl)oxazole (PIPOP). Prepared according to the general van Leusen oxazole synthesis procedure using P4 (41 mg, $0.10 \mathrm{mmol})$, T1 (38 $\mathrm{mg}, 0.12 \mathrm{mmol}), \mathrm{K}_{2} \mathrm{CO}_{3}(41 \mathrm{mg}, 0.30 \mathrm{mmol}), \mathrm{MeOH}(3 \mathrm{~mL})$ and DME $(1 \mathrm{~mL})$. Pale white solid (46 mg, 81\%). ${ }^{1} \mathrm{H}$ NMR $\left(500 \mathrm{MHz}, \mathrm{CDCl}_{3}\right) \delta 7.92(\mathrm{~s}, 1 \mathrm{H})$, $7.63(\mathrm{~s}, 1 \mathrm{H}), 7.60(\mathrm{~d}, J=8.5 \mathrm{~Hz}, 2 \mathrm{H}), 7.53(\mathrm{~d}, J=8.6 \mathrm{~Hz}, 2 \mathrm{H}), 7.49(\mathrm{~d}, J=8.6 \mathrm{~Hz}, 2 \mathrm{H}), 7.35(\mathrm{~d}$, $J=8.4 \mathrm{~Hz}, 2 \mathrm{H}), 7.28-7.22(\mathrm{~m}, 3 \mathrm{H}), 3.80$ (t, $J=7.3 \mathrm{~Hz}, 2 \mathrm{H}), 2.57$ (s, 3H), 2.53 (s, 3H), 1.61 (d, $J=6.6 \mathrm{~Hz}, 2 \mathrm{H}), 1.25(\mathrm{~d}, J=30.5 \mathrm{~Hz}, 10 \mathrm{H}), 0.89(\mathrm{t}, J=7.1 \mathrm{~Hz}, 3 \mathrm{H}) .{ }^{13} \mathrm{C} \mathrm{NMR}\left(125 \mathrm{MHz}, \mathrm{CDCl}_{3}\right)$ $\delta$ 149.57, 145.86, 138.47, 136.90, 135.31, 131.07, 128.94, 128.17, 126.62, 126.60, 126.42, 26.34, 45.20, 31.71, 30.82, 29.02, 28.92, 26.45, 22.61, 15.61, 15.22, 14.08. HRMS (ESI): Exact mass calcd for $\mathrm{C}_{34} \mathrm{H}_{38} \mathrm{~N}_{3} \mathrm{O}_{1} \mathrm{~S}_{2}[\mathrm{M}+\mathrm{H}]^{+}$568.2456, found 568.2457.

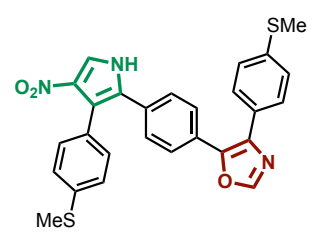

4-(4-(methylthio)phenyl)-5-(4-(3-(4-(methylthio)phenyl)-4-nitro-1Hpyrrol-2-yl)phenyl)oxazole (PRPOP). Prepared according to the general van Leusen oxazole synthesis procedure using P6 (36 mg, $0.11 \mathrm{mmol})$, T1 (41 mg, $0.13 \mathrm{mmol}), \mathrm{K}_{2} \mathrm{CO}_{3}(46 \mathrm{mg}, 0.33 \mathrm{mmol}), \mathrm{MeOH}(3 \mathrm{~mL})$ and DME $(1 \mathrm{~mL})$. Yellow solid (30 mg, 55\%). ${ }^{1} \mathrm{H}$ NMR (500 MHz, $\left.\mathrm{CDCl}_{3}\right) \delta 8.74$ (s, $\left.1 \mathrm{H}\right), 7.96$ (s, 1H), $7.87(\mathrm{~d}, J=3.5 \mathrm{~Hz}, 1 \mathrm{H}), 7.56(\mathrm{dd}, J=8.5,5.6 \mathrm{~Hz}, 4 \mathrm{H}), 7.28-7.23(\mathrm{~m}, 6 \mathrm{H}), 7.17(\mathrm{~d}, J=$ $8.6 \mathrm{~Hz}, 2 \mathrm{H}), 2.54(\mathrm{~d}, J=4.0 \mathrm{~Hz}, 6 \mathrm{H}) .{ }^{13} \mathrm{C} \mathrm{NMR}\left(125 \mathrm{MHz}, \mathrm{CDCl}_{3}\right) \delta 149.96,144.77,139.22$, 138.16, 136.70, 135.08, 131.11, 130.90, 129.67, 128.49, 128.32, 128.21, 128.15, 127.37, 126.85, 126.30, 126.06, 120.58, 117.71, 15.51, 15.48. HRMS (ESI): Exact mass calcd for $\mathrm{C}_{27} \mathrm{H}_{22} \mathrm{~N}_{3} \mathrm{O}_{3} \mathrm{~S}_{2}$ $[\mathrm{M}+\mathrm{H}]^{+}$500.1103, found 500.1084.

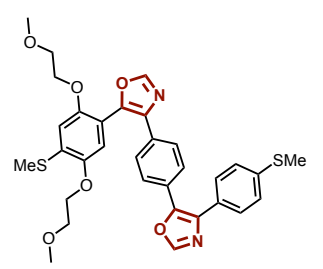

5-(2,5-bis(2-methoxyethoxy)-4-(methylthio)phenyl)-4-(4-(4-(4(methylthio)phenyl)oxazol-5-yl)phenyl)oxazole (GOPOP). Prepared according to the general van Leusen oxazole synthesis procedure using $\mathbf{O 2}$ (33 mg, $0.075 \mathrm{mmol}$ ), T1 (44 mg, $0.09 \mathrm{mmol}), \mathrm{K}_{2} \mathrm{CO}_{3}(31 \mathrm{mg}, 0.23 \mathrm{mmol}), \mathrm{MeOH}$ (3 mL) and DME (1 mL). Yellow solid (34 mg, 61\%). ${ }^{1} \mathrm{H}$ NMR (500 MHz, $\left.\mathrm{CDCl}_{3}\right) \delta 8.00(\mathrm{~s}, 1 \mathrm{H}), 7.96(\mathrm{~s}, 1 \mathrm{H}), 7.65-7.58(\mathrm{~m}, 6 \mathrm{H}), 7.27(\mathrm{~d}, J=8.4 \mathrm{~Hz}$, $2 \mathrm{H}), 7.00(\mathrm{~s}, 1 \mathrm{H}), 6.83(\mathrm{~s}, 1 \mathrm{H}), 4.16-4.13(\mathrm{~m}, 2 \mathrm{H}), 3.96-3.93(\mathrm{~m}, 2 \mathrm{H}), 3.80-3.77(\mathrm{~m}, 2 \mathrm{H}), 3.48$ $(\mathrm{s}, 3 \mathrm{H}), 3.22(\mathrm{~s}, 3 \mathrm{H}), 3.21-3.17(\mathrm{~m}, 2 \mathrm{H}), 2.53(\mathrm{~s}, 3 \mathrm{H}), 2.50(\mathrm{~s}, 3 \mathrm{H}) .{ }^{13} \mathrm{C} \mathrm{NMR}\left(125 \mathrm{MHz}, \mathrm{CDCl}_{3}\right)$ $\delta 151.18,150.00,149.81,142.41,137.92,135.24,131.58,129.46,127.23,126.10,114.98,114.02$, 111.59, 70.97, 70.60, 69.27, 69.01, 59.38, 59.13, 15.78, 14.49. HRMS (ESI): Exact mass calcd for $\mathrm{C}_{32} \mathrm{H}_{33} \mathrm{~N}_{2} \mathrm{O}_{6} \mathrm{~S}_{2}[\mathrm{M}+\mathrm{H}]^{+} 605.1780$, found 605.1768 .

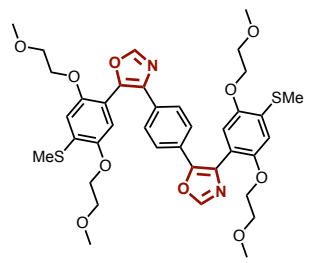

4-(2,5-bis(2-methoxyethoxy)-4-(methylthio)phenyl)-5-(4-(5-(2,5-bis(2-
methoxyethoxy)-4-(methylthio)phenyl)oxazol-4-yl)phenyl)oxazole (GOPOG). Prepared according to the general van Leusen oxazole synthesis procedure using $\mathbf{O 2}$ (33 mg, $0.075 \mathrm{mmol}$ ), T2 (44 mg, $0.09 \mathrm{mmol}$ ), $\mathrm{K}_{2} \mathrm{CO}_{3}$ (31 $\mathrm{mg}, 0.23 \mathrm{mmol}), \mathrm{MeOH}(3 \mathrm{~mL})$ and DME (1 mL). Yellow solid (34 mg, 61\%). ${ }^{1} \mathrm{H}$ NMR (500 MHz, $\left.\mathrm{CDCl}_{3}\right) \delta 7.99(\mathrm{~s}, 1 \mathrm{H}), 7.97(\mathrm{~s}, 1 \mathrm{H}), 7.58(\mathrm{~d}, J=8.5 \mathrm{~Hz}$, $2 \mathrm{H}), 7.45(\mathrm{~d}, J=8.5 \mathrm{~Hz}, 2 \mathrm{H}), 7.08(\mathrm{~s}, 1 \mathrm{H}), 6.96(\mathrm{~s}, 1 \mathrm{H}), 6.82(\mathrm{~s}, 1 \mathrm{H}), 6.81(\mathrm{~s}, 1 \mathrm{H}), 4.21-4.16(\mathrm{~m}$, $2 \mathrm{H}), 4.15-4.09(\mathrm{~m}, 2 \mathrm{H}), 3.94-3.90(\mathrm{~m}, 2 \mathrm{H}), 3.90-3.86(\mathrm{~m}, 2 \mathrm{H}), 3.81-3.77(\mathrm{~m}, 4 \mathrm{H}), 3.48(\mathrm{~d}$, $J=3.9 \mathrm{~Hz}, 6 \mathrm{H}), 3.20(\mathrm{~s}, 3 \mathrm{H}), 3.16(\mathrm{~s}, 3 \mathrm{H}), 2.49(\mathrm{~d}, J=3.1 \mathrm{~Hz}, 6 \mathrm{H}) .{ }^{13} \mathrm{C} \mathrm{NMR}\left(125 \mathrm{MHz}, \mathrm{CDCl}_{3}\right)$ $\delta 150.05,149.78,149.38,146.65,143.04,131.99,126.56,125.35,114.63,114.11,111.91,111.48$, 71.04, 70.98, 70.64, 70.61, 69.36, 69.17, 69.00, 68.85, 59.38, 59.34, 59.10, 59.02, 14.67, 14.45. HRMS (ESI): Exact mass calcd for $\mathrm{C}_{38} \mathrm{H}_{45} \mathrm{~N}_{2} \mathrm{O}_{10} \mathrm{~S}_{2}[\mathrm{M}+\mathrm{H}]^{+} 753.2516$, found 753.2518. 


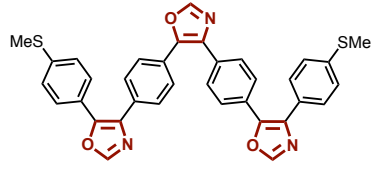

4-(4-(methylthio)phenyl)-5-(4-(5-(4-(5-(4(methylthio)phenyl)oxazol-4-yl)phenyl)oxazol-4-yl)phenyl)oxazole (POPOPOP). Prepared according to the general van Leusen oxazole synthesis procedure using $\mathbf{H 2}(15 \mathrm{mg}, 0.03 \mathrm{mmol}), \mathbf{T 1}(13 \mathrm{mg}, 0.04$

$\mathrm{mmol}), \mathrm{K}_{2} \mathrm{CO}_{3}(13 \mathrm{mg}, 0.09 \mathrm{mmol}), \mathrm{MeOH}(1.5 \mathrm{~mL})$ and DME $(0.5 \mathrm{~mL})$. White solid $(14 \mathrm{mg}$, 78\%). ${ }^{1} \mathrm{H}$ NMR $\left(500 \mathrm{MHz}, \mathrm{CDCl}_{3}\right) \delta 7.92(\mathrm{~s}, 1 \mathrm{H}), 7.89(\mathrm{~d}, J=1.9 \mathrm{~Hz}, 2 \mathrm{H}), 7.65(\mathrm{dd}, J=8.3,3.4$ $\mathrm{Hz}, 4 \mathrm{H}), 7.58(\mathrm{dd}, J=8.3,4.8 \mathrm{~Hz}, 4 \mathrm{H}), 7.54(\mathrm{~d}, J=8.3 \mathrm{~Hz}, 2 \mathrm{H}), 7.47$ (d, J=8.4 Hz, 2H), 7.17 (s, $1 \mathrm{H}), 2.44(\mathrm{~d}, J=0.8 \mathrm{~Hz}, 6 \mathrm{H}) .{ }^{13} \mathrm{C} \mathrm{NMR}\left(125 \mathrm{MHz}, \mathrm{CDCl}_{3}\right) \delta 150.00,149.87,149.81,140.37$, $138.98,134.83,133.66,132.45,128.67,128.52$, 128.33, 128.18, 128.06, 127.30, 127.01, 126.80, 126.38, 126.14, 124.88, 15.55, 15.27. HRMS (ESI): Exact mass calcd for $\mathrm{C}_{35} \mathrm{H}_{26} \mathrm{~N}_{3} \mathrm{O}_{3} \mathrm{~S}_{2}[\mathrm{M}+\mathrm{H}]^{+}$ 600.1414 , found 600.1416 . 
3. ${ }^{1} \mathrm{H}$ and ${ }^{13} \mathrm{C}$ Spectra

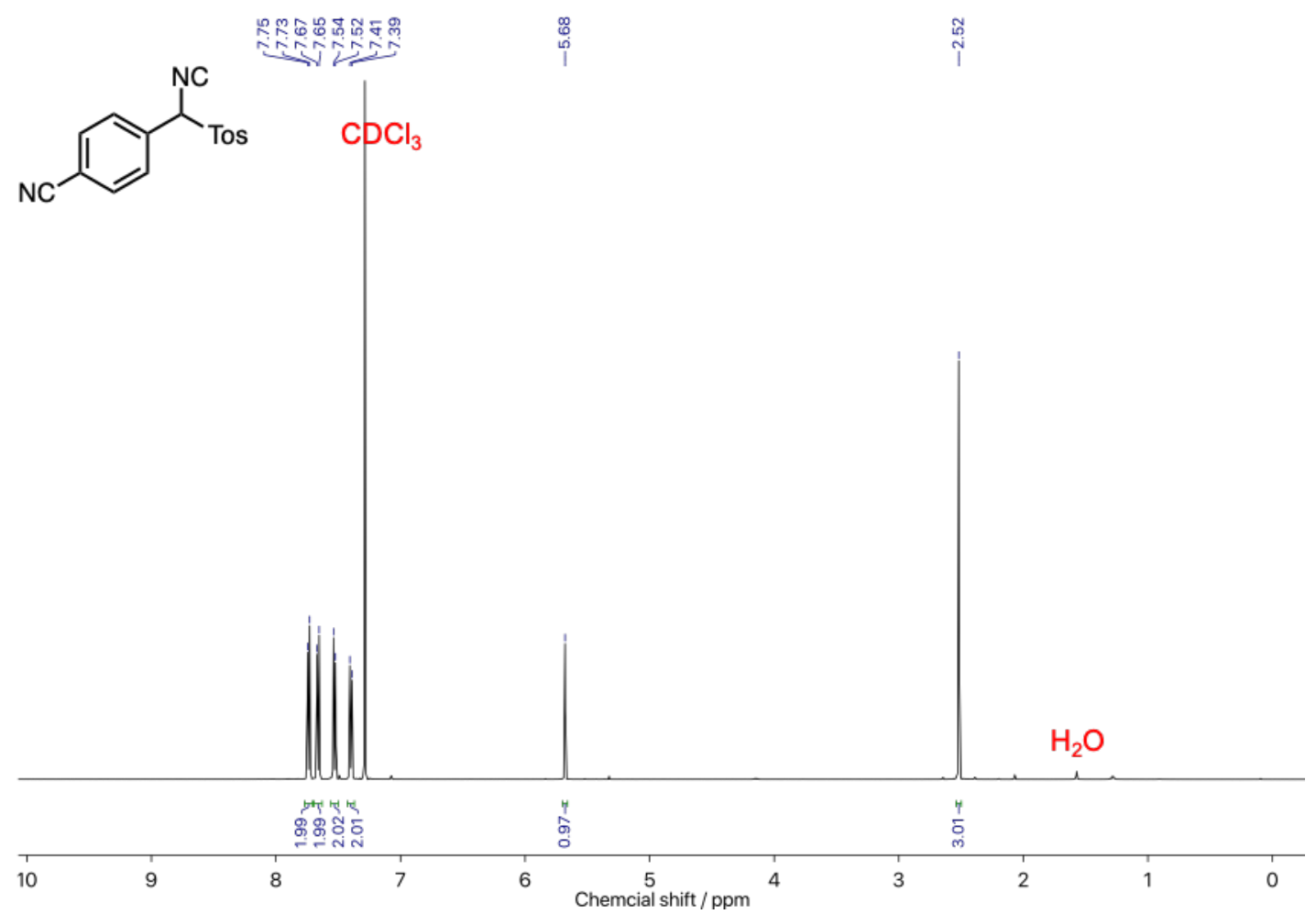

Figure S7. ${ }^{1} \mathrm{H} \mathrm{NMR}$ spectrum of $\mathbf{M}\left(500 \mathrm{MHz}, \mathrm{CDCl}_{3}\right)$.

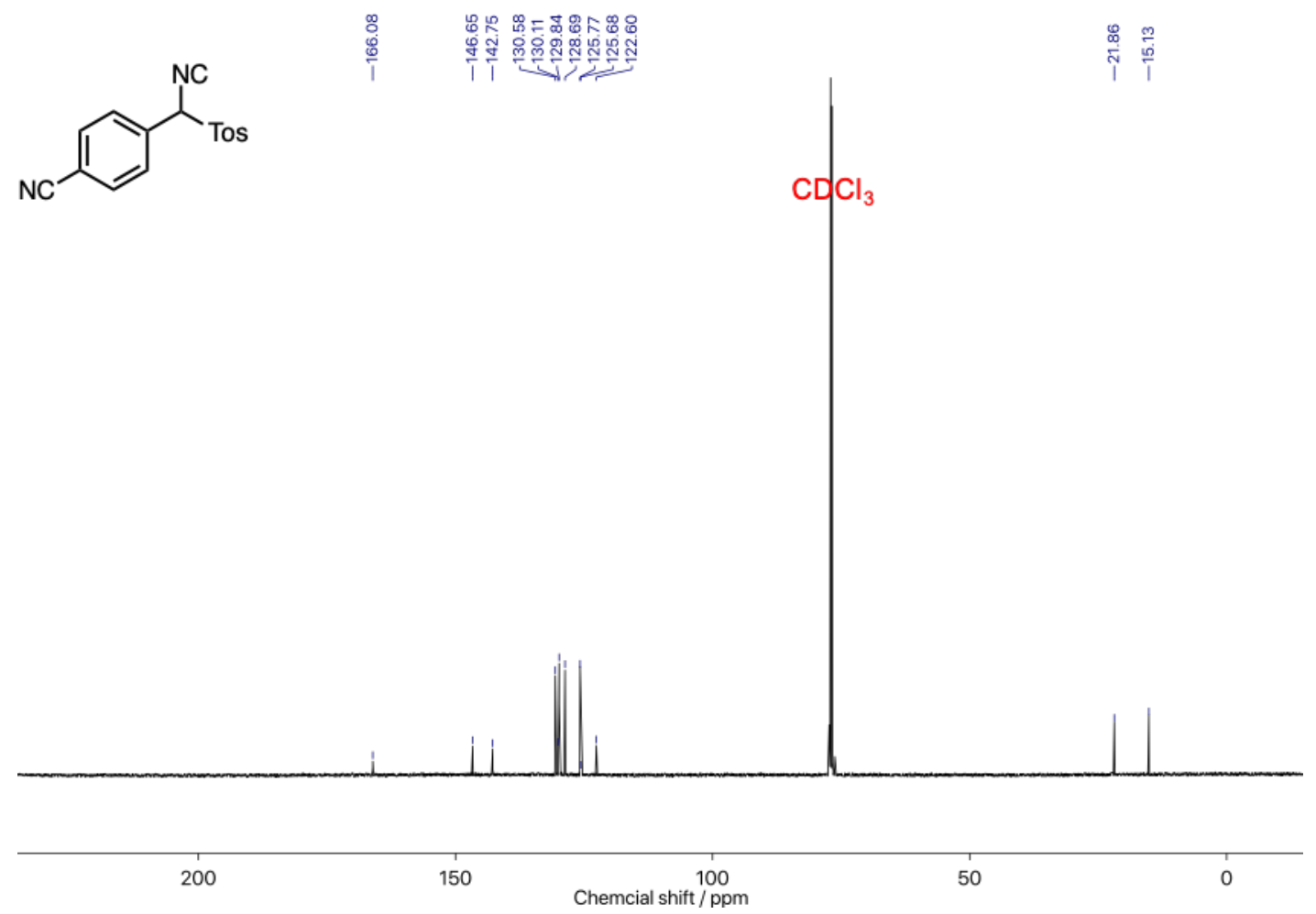

Figure S8. ${ }^{13} \mathrm{C}$ NMR spectrum of $\mathbf{M}\left(125 \mathrm{MHz}, \mathrm{CDCl}_{3}\right)$. 
<smiles>CSc1ccc(C(C)(N)C2CC2)cc1</smiles>

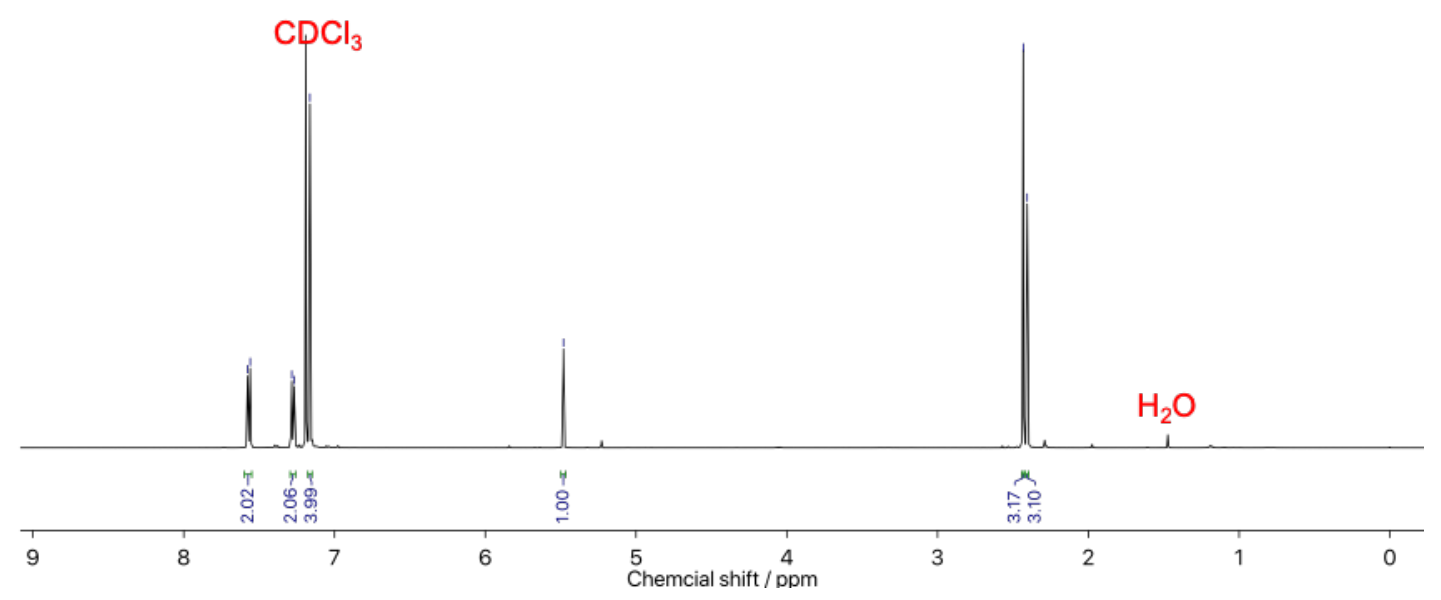

Figure S9. ${ }^{1} \mathrm{H} \mathrm{NMR}$ spectrum of $\mathbf{T} 1\left(500 \mathrm{MHz}, \mathrm{CDCl}_{3}\right)$.

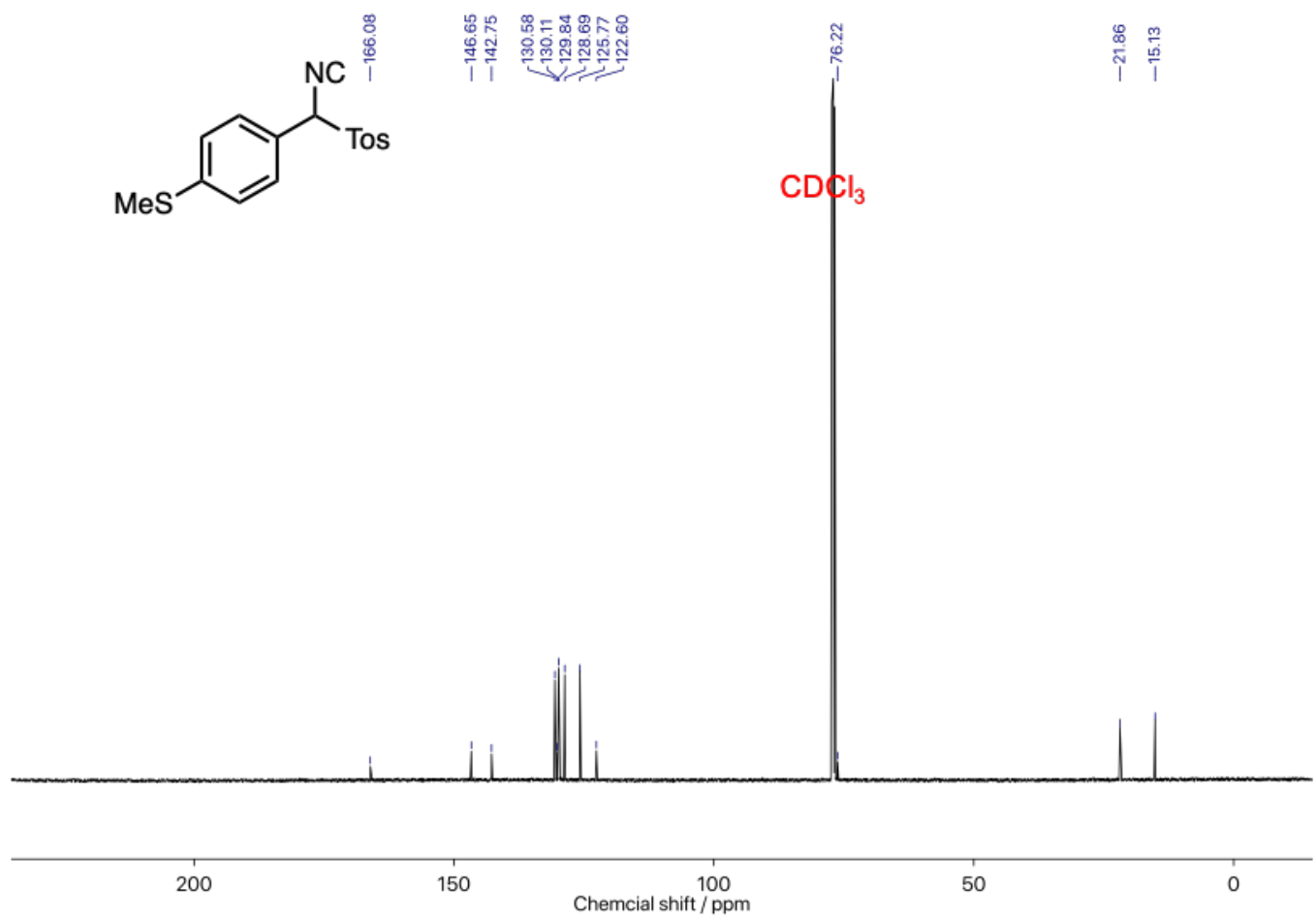

Figure S10. ${ }^{13} \mathrm{C}$ NMR spectrum of T1 $\left(125 \mathrm{MHz}, \mathrm{CDCl}_{3}\right)$. 


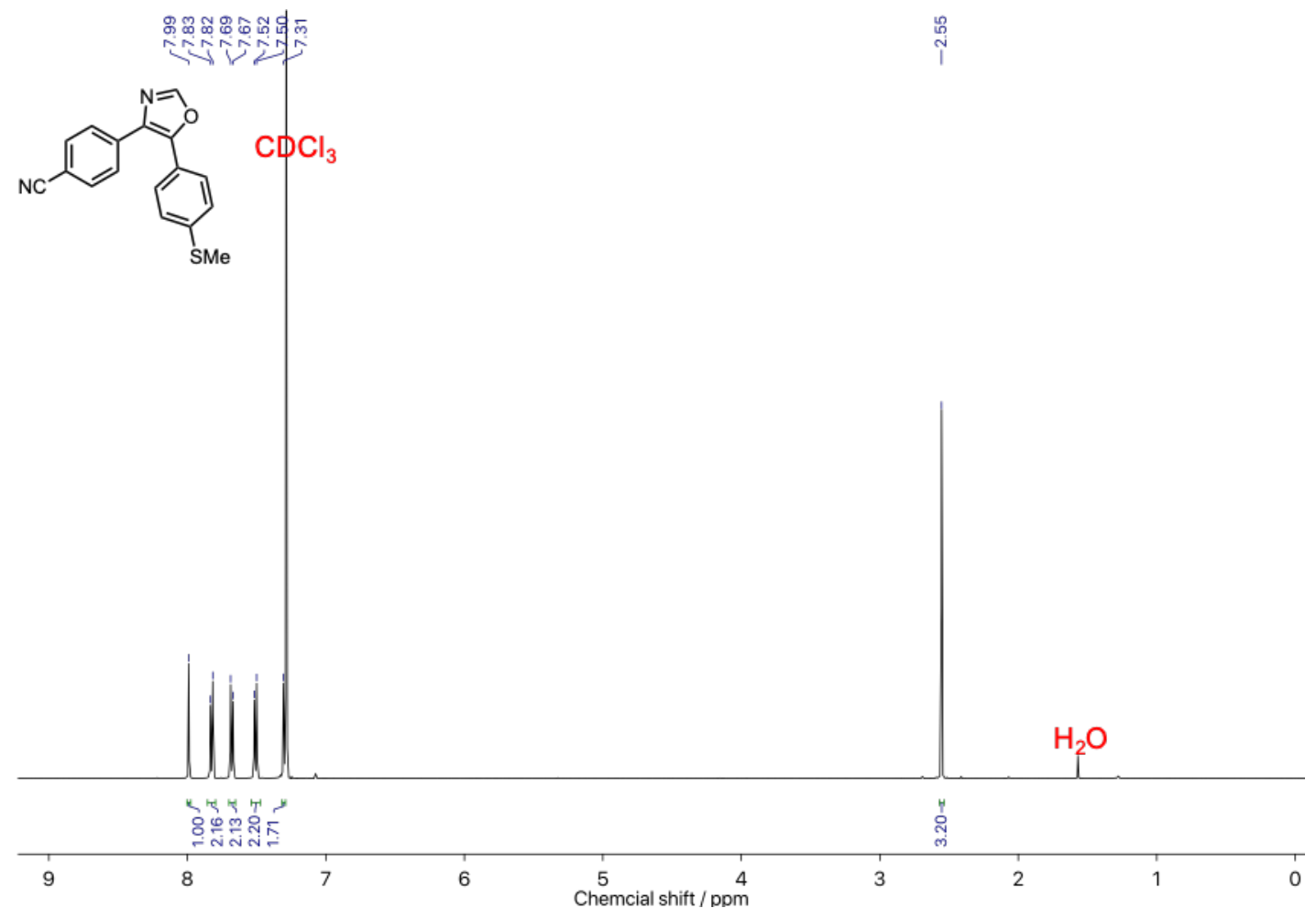

Figure S11. ${ }^{1} \mathrm{H} \mathrm{NMR}$ spectrum of $\mathbf{P 1}\left(500 \mathrm{MHz}, \mathrm{CDCl}_{3}\right)$.
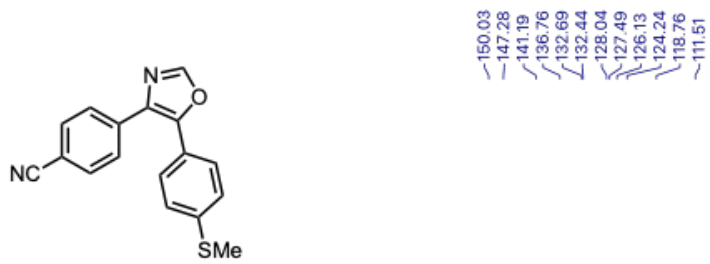

$\mathrm{CфCl}_{3}$

$\frac{\overrightarrow{0}}{1}$

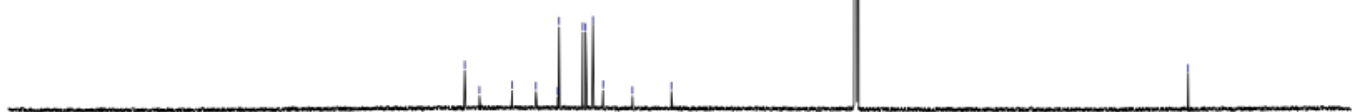

200

150

Chemcial shift $/ \mathrm{ppm}$

50

0

Figure S12. ${ }^{13} \mathrm{C}$ NMR spectrum of $\mathbf{P 1}\left(125 \mathrm{MHz}, \mathrm{CDCl}_{3}\right)$. 


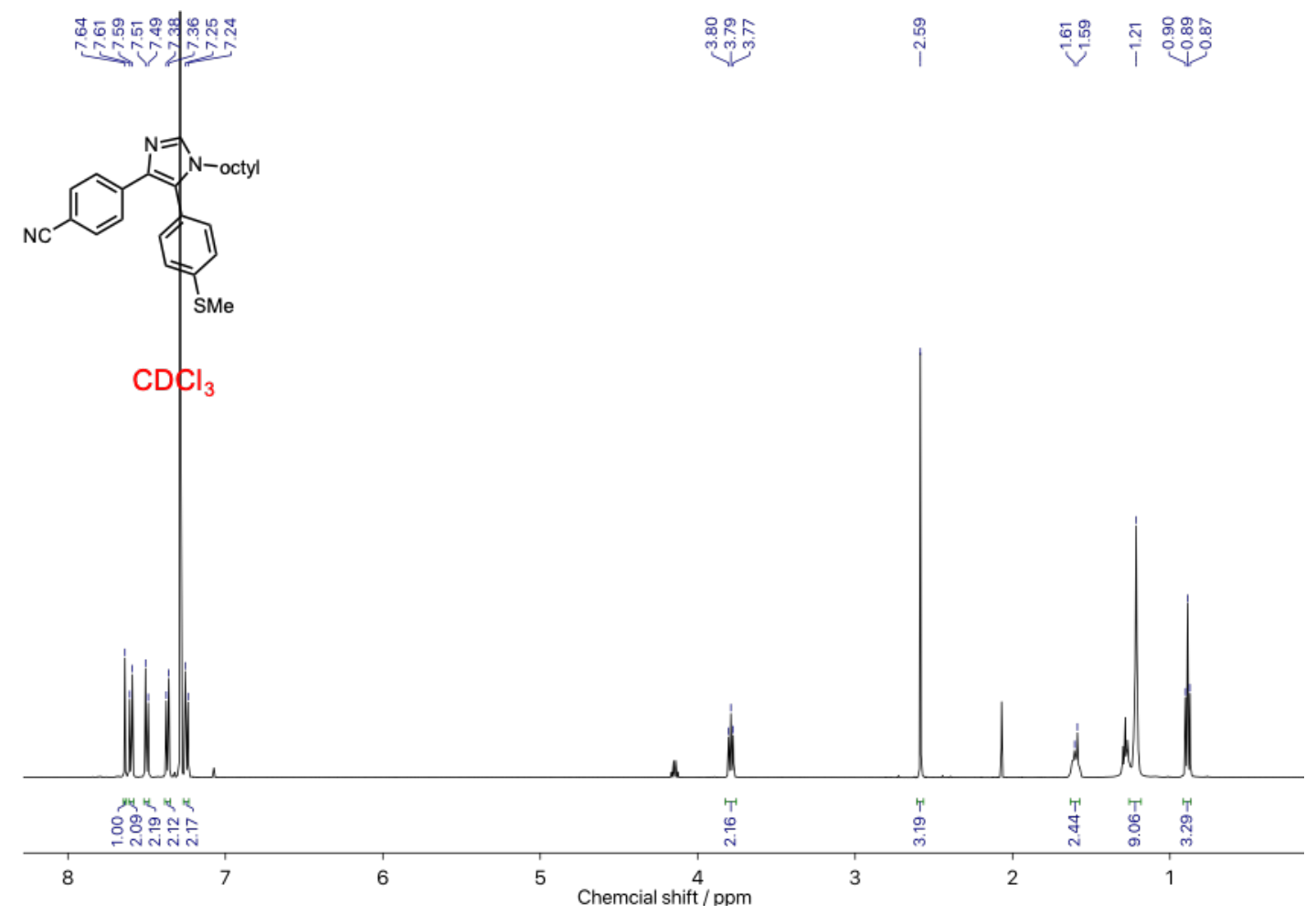

Figure S13. ${ }^{1} \mathrm{H}$ NMR spectrum of $\mathbf{P 3}\left(500 \mathrm{MHz}, \mathrm{CDCl}_{3}\right)$.

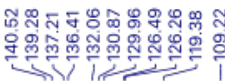<smiles>Cc1ccc(-c2nn(C)c(O)c2-c2ccc(C)cc2)cc1</smiles>
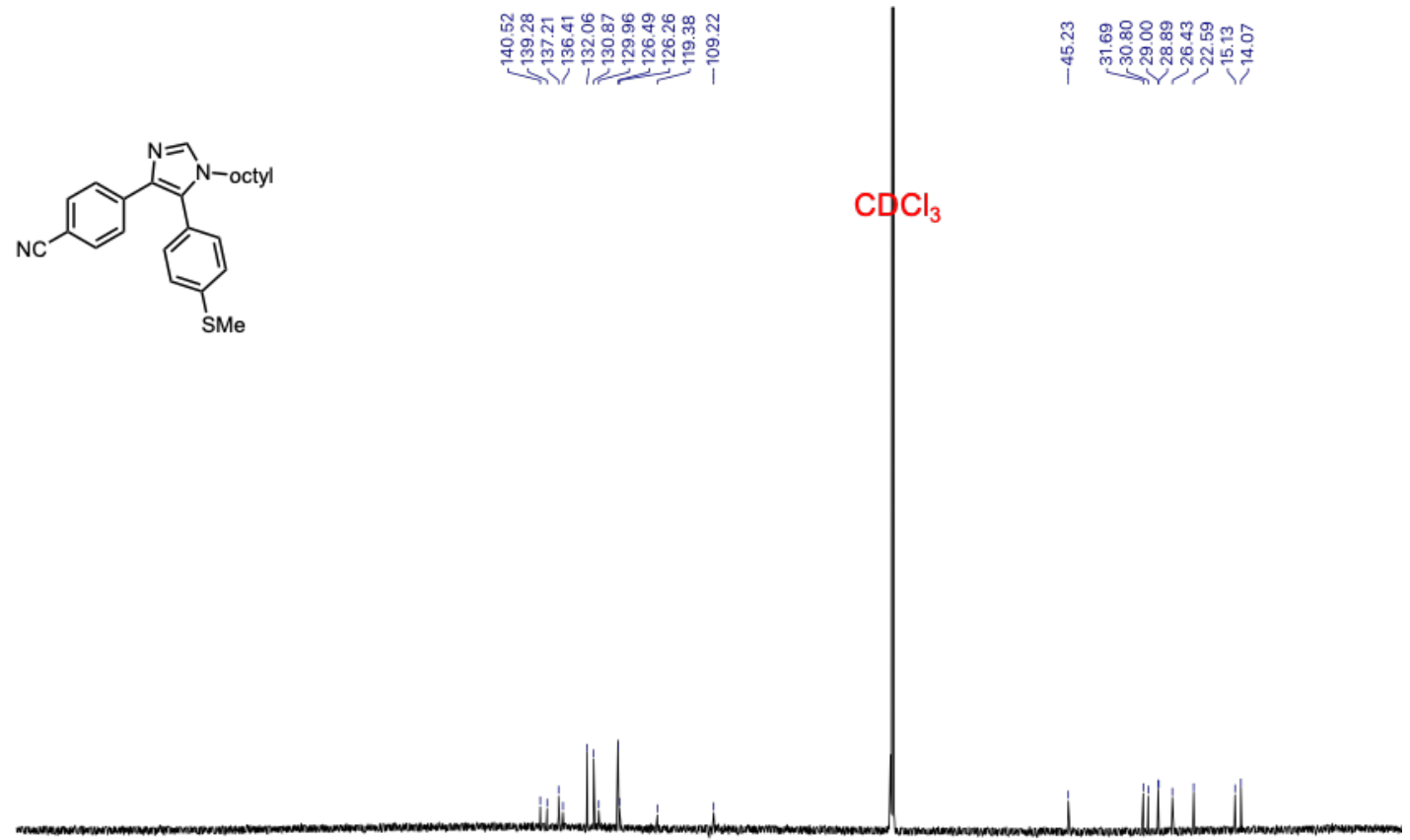

200

150

$\stackrel{100}{\text { Chemcial shift / ppm }}$

50

0

Figure S14. ${ }^{13} \mathrm{C}$ NMR spectrum of $\mathbf{P 3}\left(125 \mathrm{MHz}, \mathrm{CDCl}_{3}\right)$. 


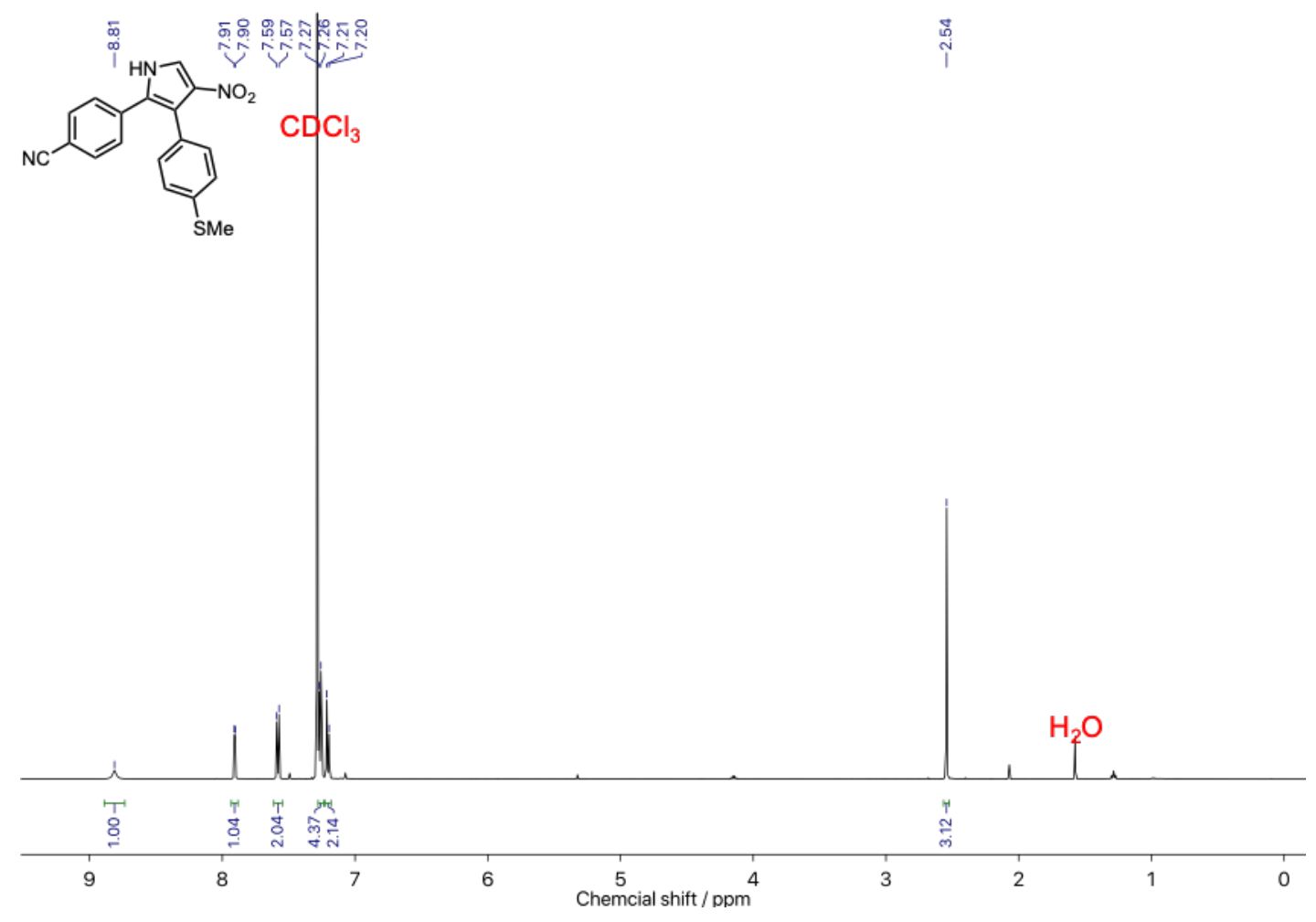

Figure S15. ${ }^{1} \mathrm{H}$ NMR spectrum of $\mathbf{P 5}\left(500 \mathrm{MHz}, \mathrm{CDCl}_{3}\right)$.
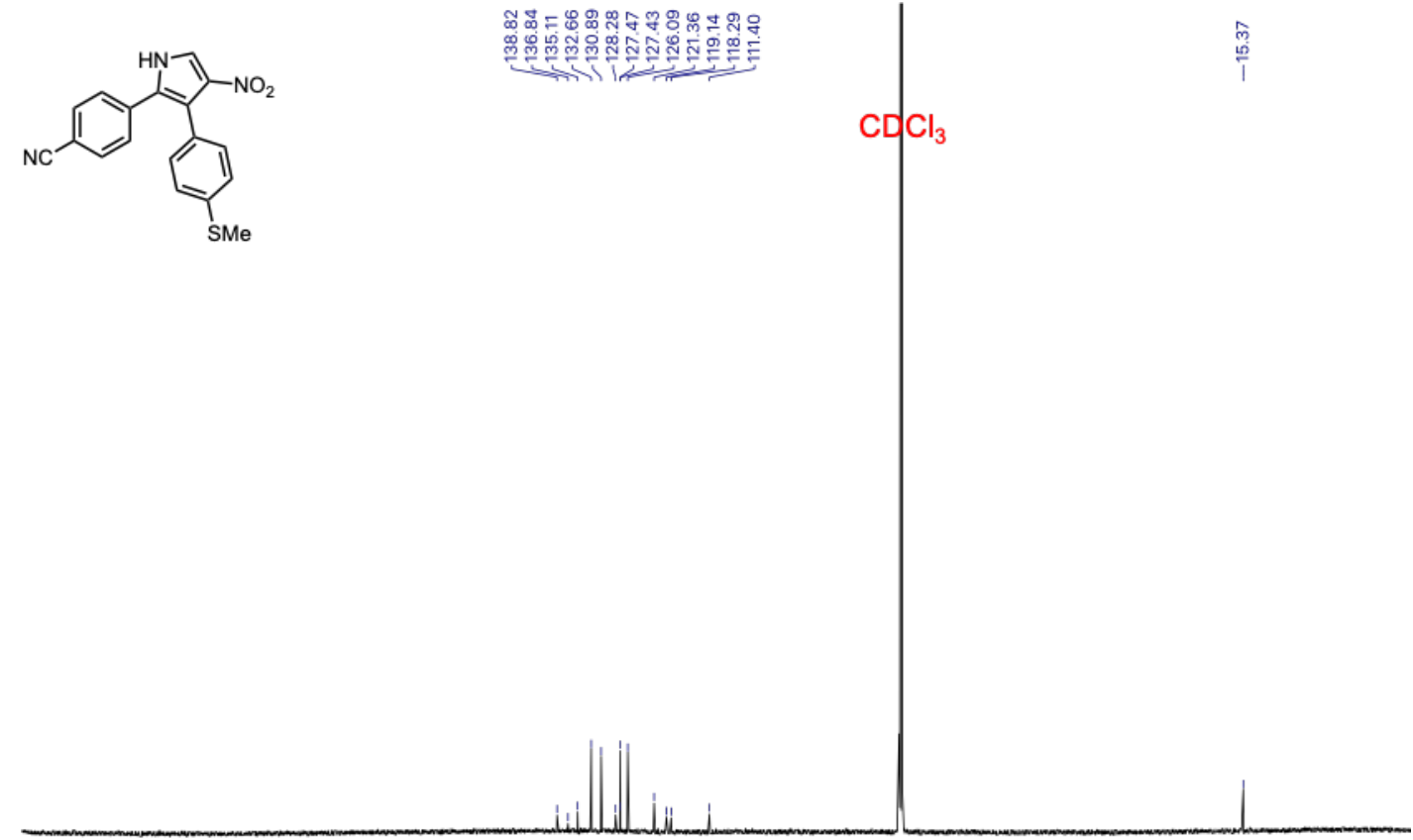

200

150

Chemcial shift / ppm

50

Figure S16. ${ }^{13} \mathrm{C}$ NMR spectrum of $\mathbf{P 5}\left(125 \mathrm{MHz}, \mathrm{CDCl}_{3}\right)$. 


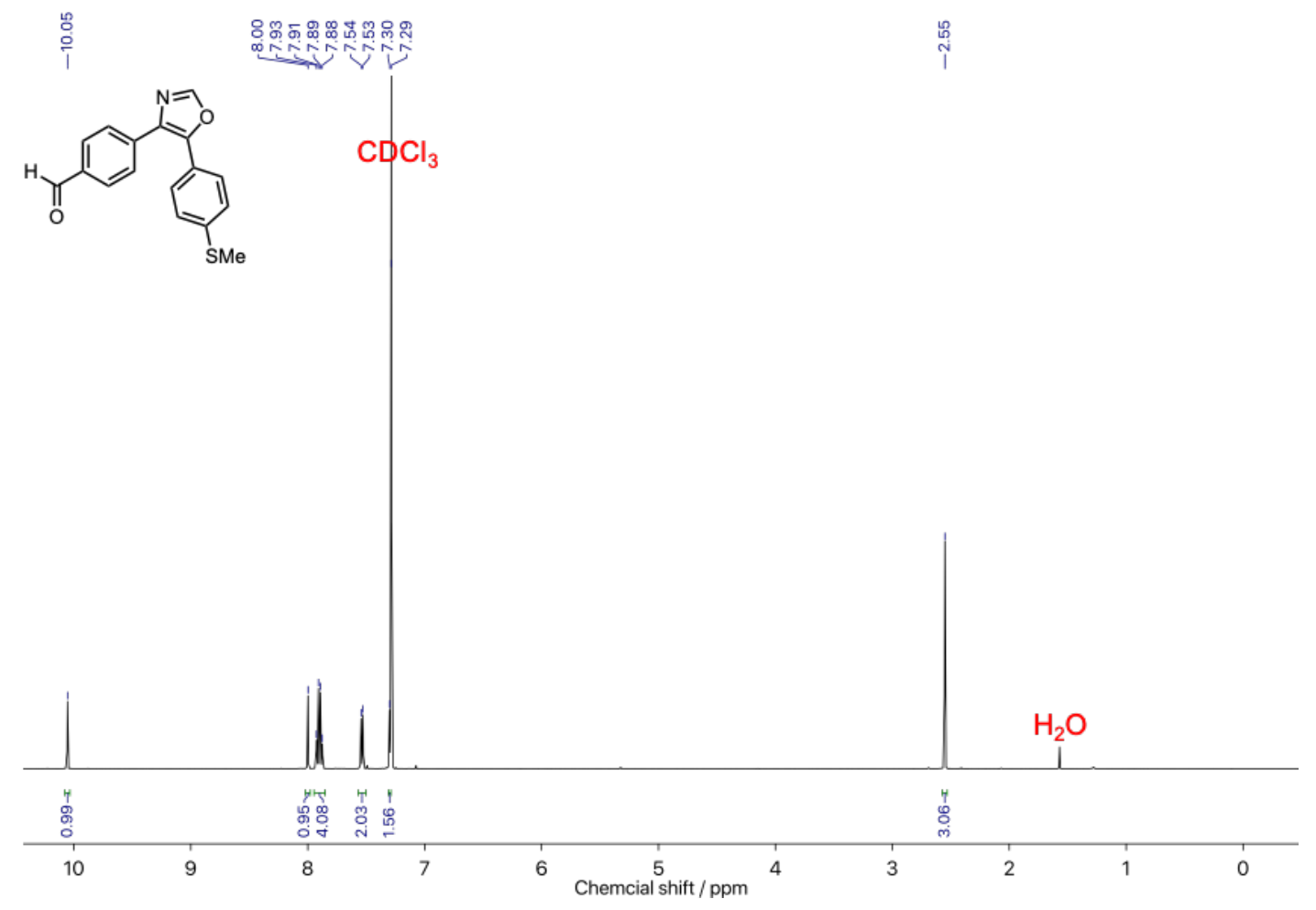

Figure S17. ${ }^{1} \mathrm{H} \mathrm{NMR}$ spectrum of $\mathbf{P 2}\left(500 \mathrm{MHz}, \mathrm{CDCl}_{3}\right)$.
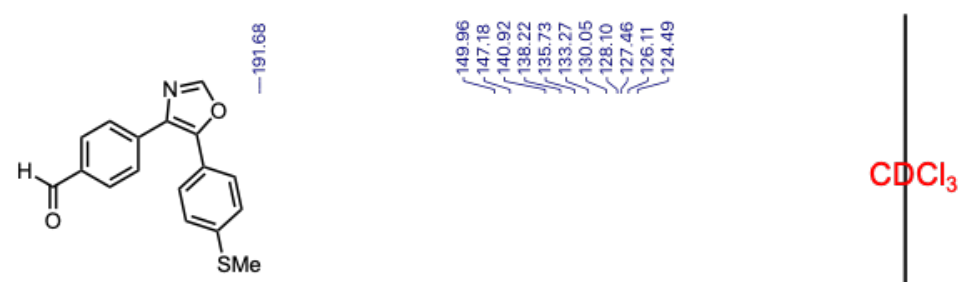

츰

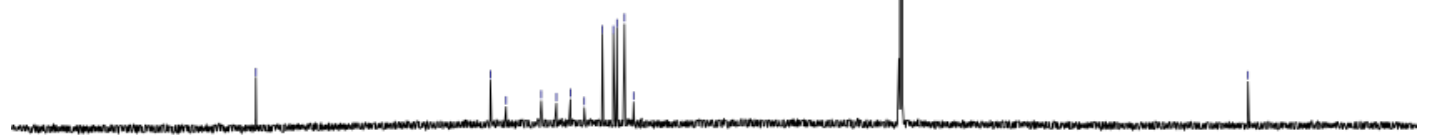

200

150

$\stackrel{100}{\text { Chemcial shift } / \mathrm{ppm}}$

50

0

Figure S18. ${ }^{13} \mathrm{C}$ NMR spectrum of $\mathbf{P 2}\left(125 \mathrm{MHz}, \mathrm{CDCl}_{3}\right)$. 


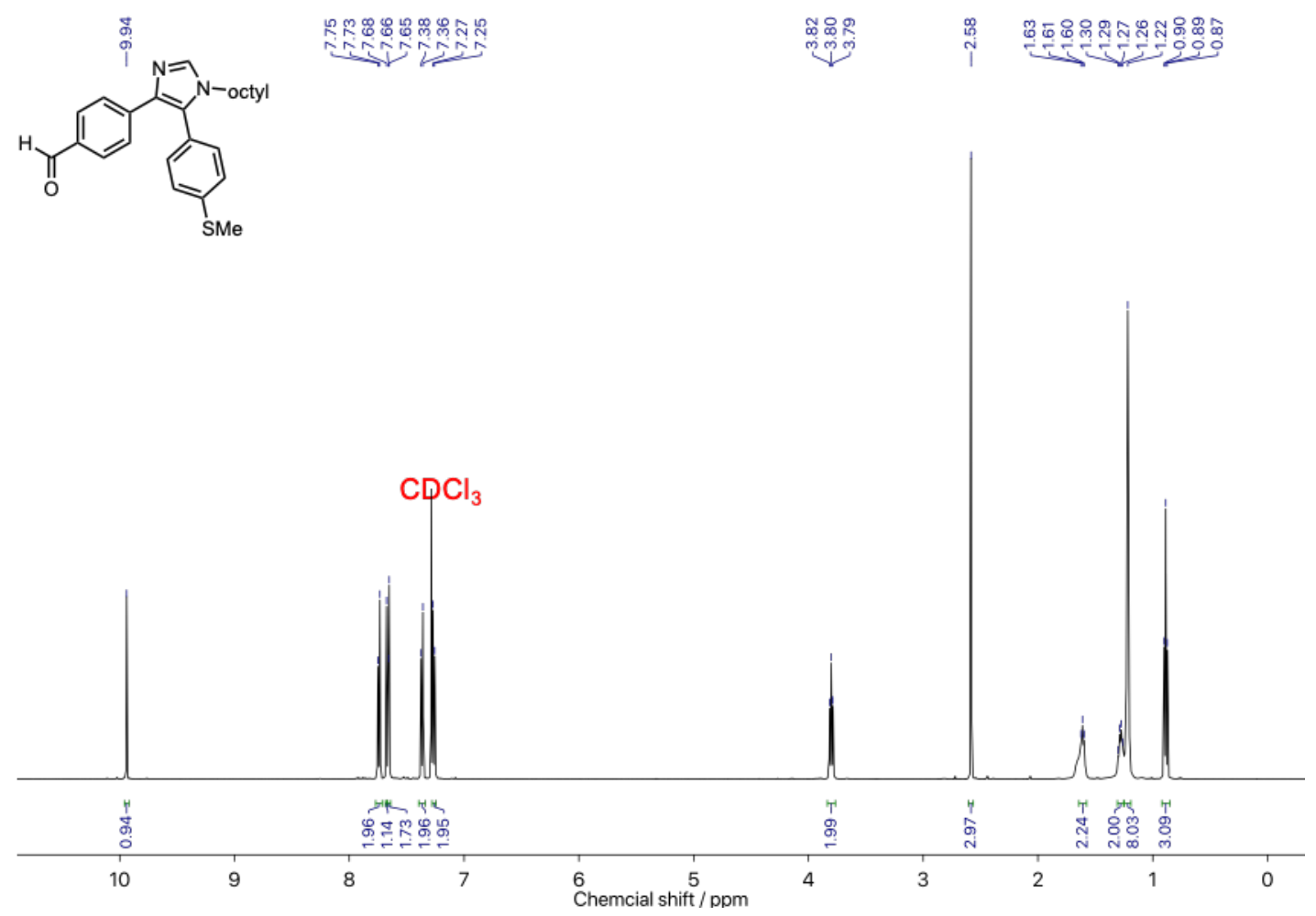

Figure S19. ${ }^{1} \mathrm{H} \mathrm{NMR}$ spectrum of $\mathbf{P} 4\left(500 \mathrm{MHz}, \mathrm{CDCl}_{3}\right)$.
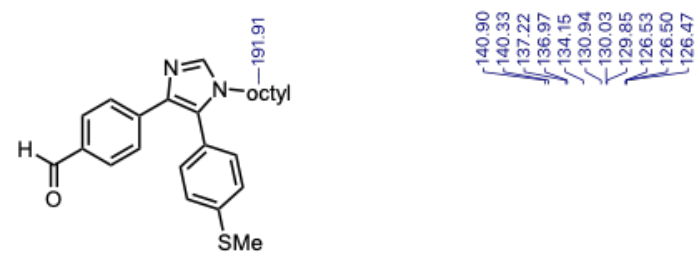

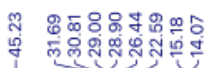

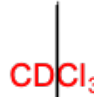

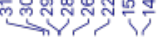

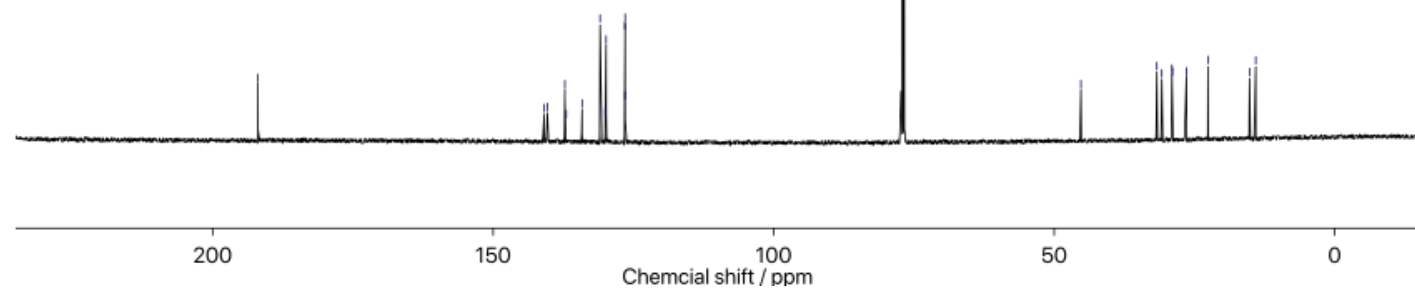

Figure S20. ${ }^{13} \mathrm{C}$ NMR spectrum of $\mathbf{P 4}\left(125 \mathrm{MHz}, \mathrm{CDCl}_{3}\right)$. 


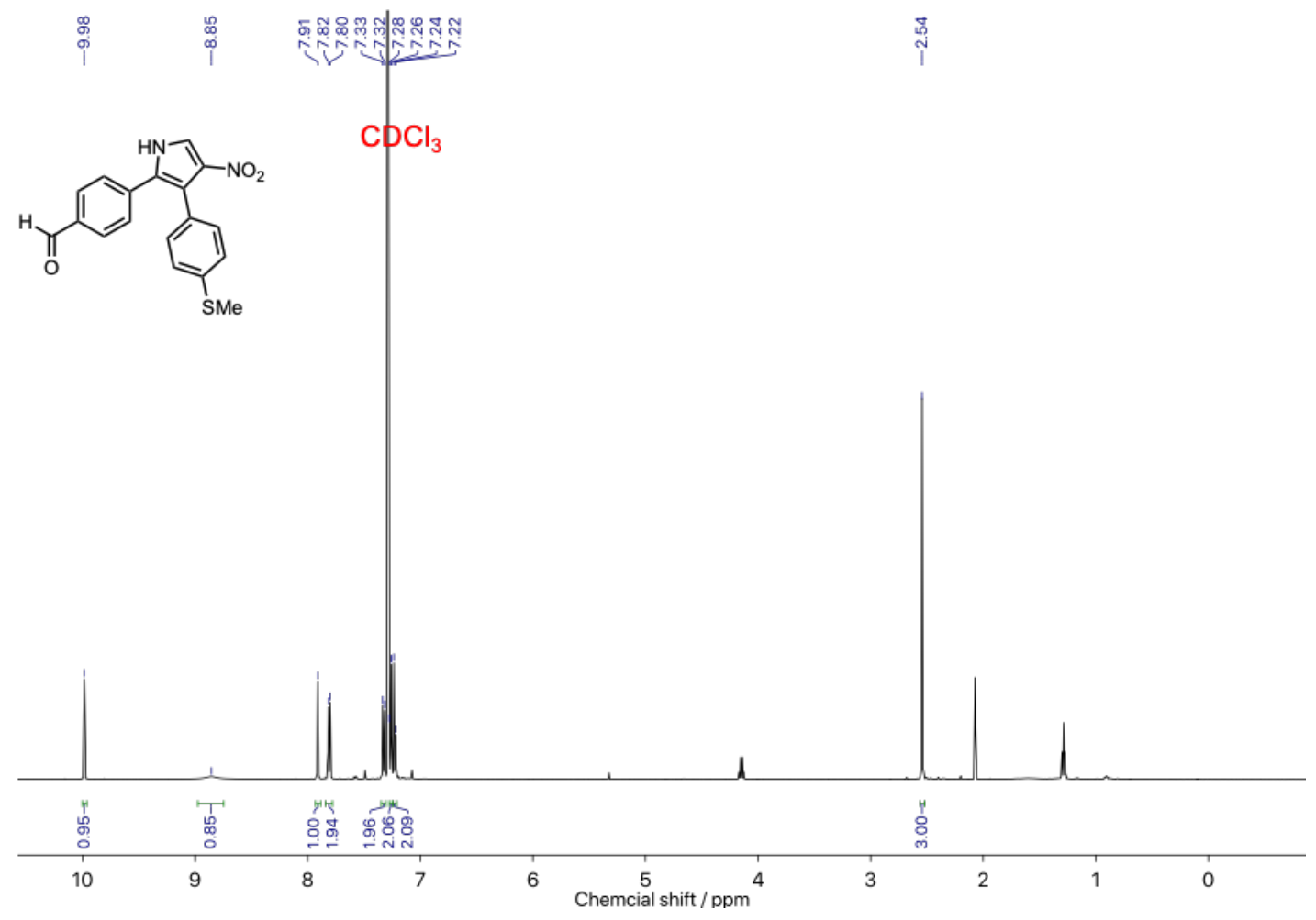

Figure S21. ${ }^{1} \mathrm{H} \mathrm{NMR}$ spectrum of $\mathbf{P 6}\left(500 \mathrm{MHz}, \mathrm{CDCl}_{3}\right)$.

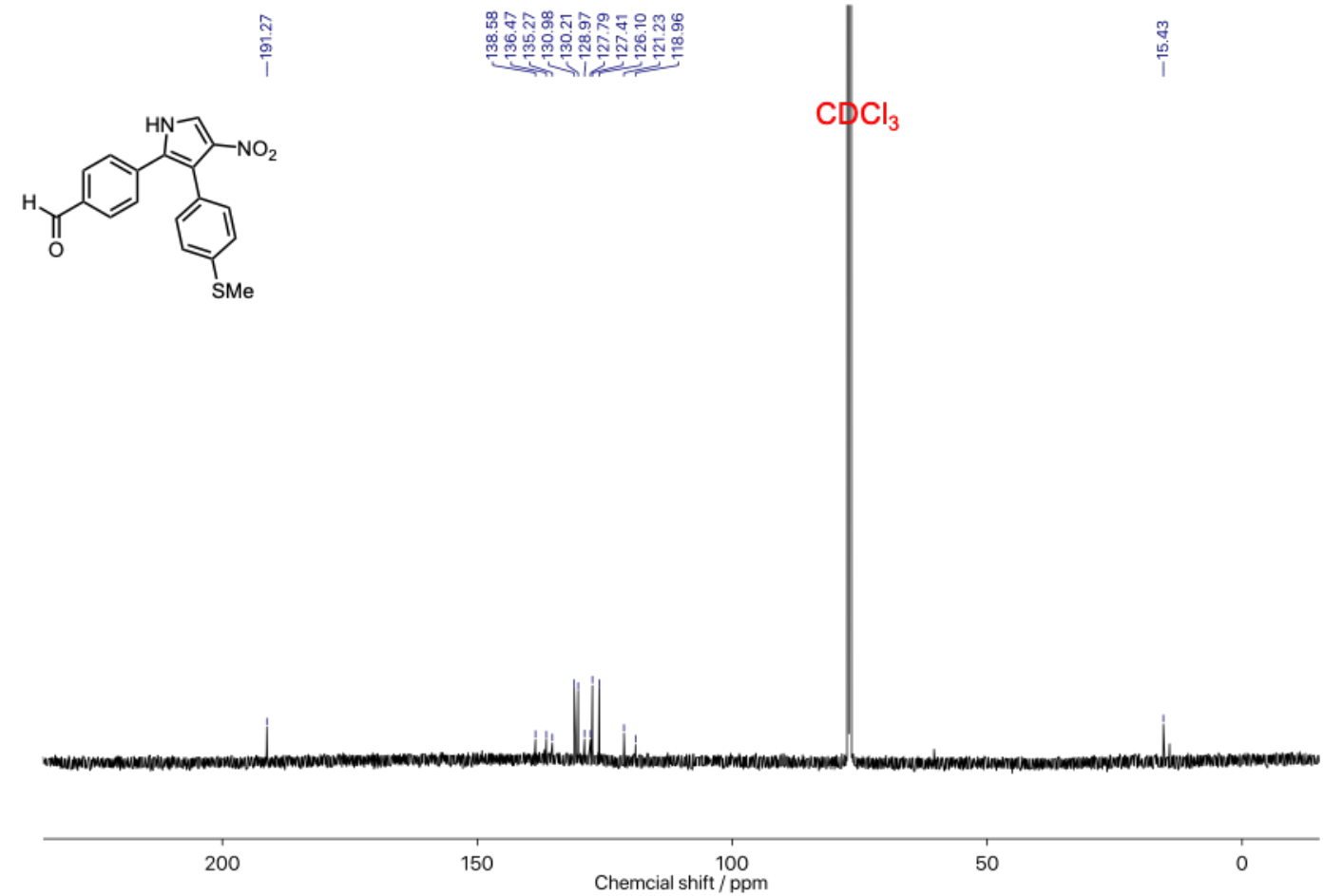

Figure S22. ${ }^{13} \mathrm{C}$ NMR spectrum of $\mathbf{P 6}\left(125 \mathrm{MHz}, \mathrm{CDCl}_{3}\right)$. 


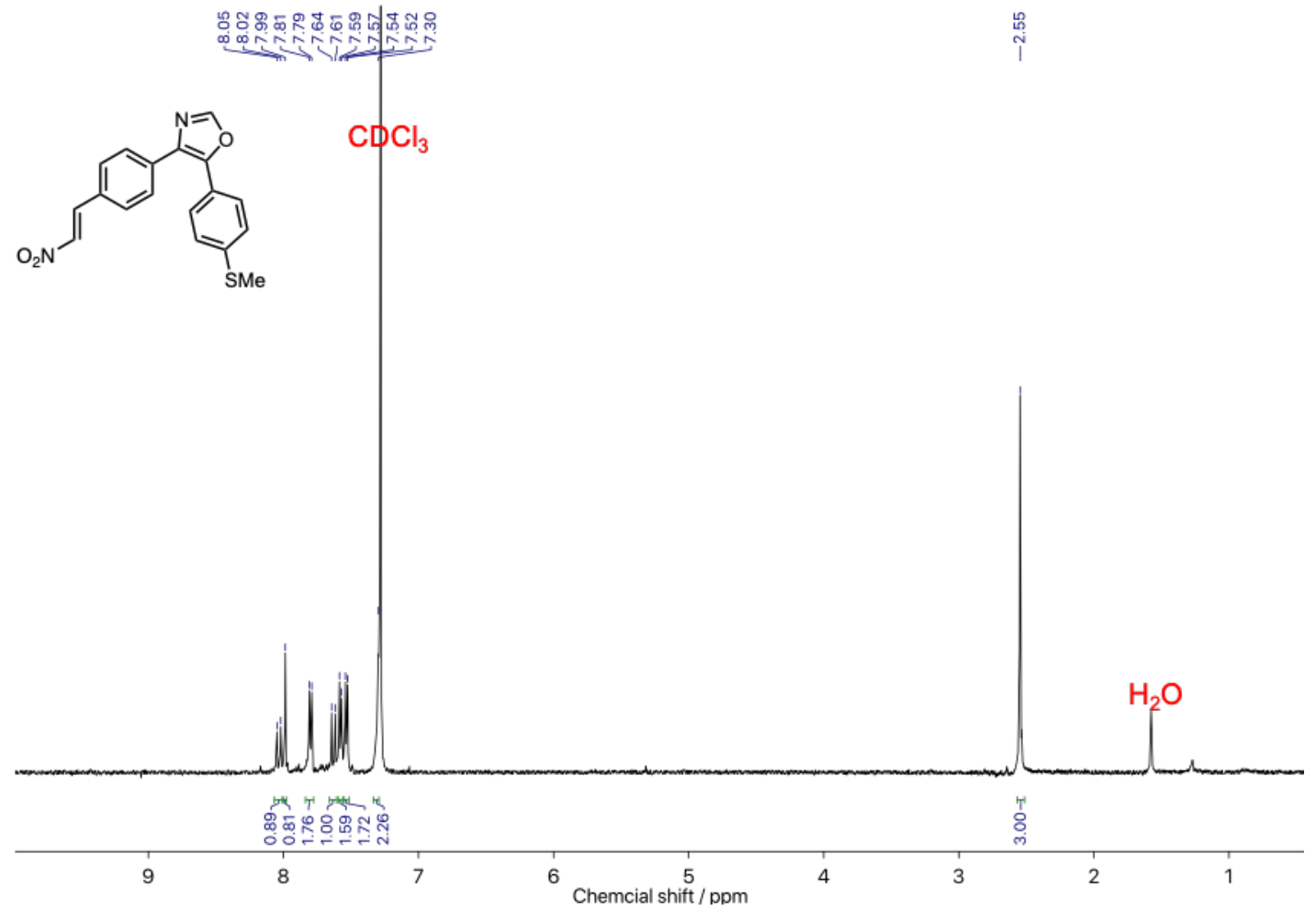

Figure S23. ${ }^{1} \mathrm{H}$ NMR spectrum of $\mathbf{P} 7\left(500 \mathrm{MHz}, \mathrm{CDCl}_{3}\right)$.
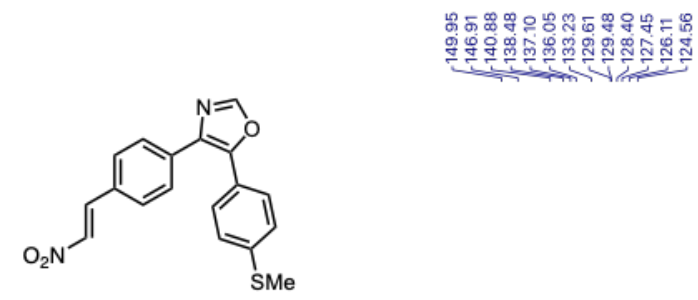

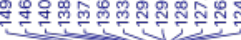

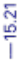

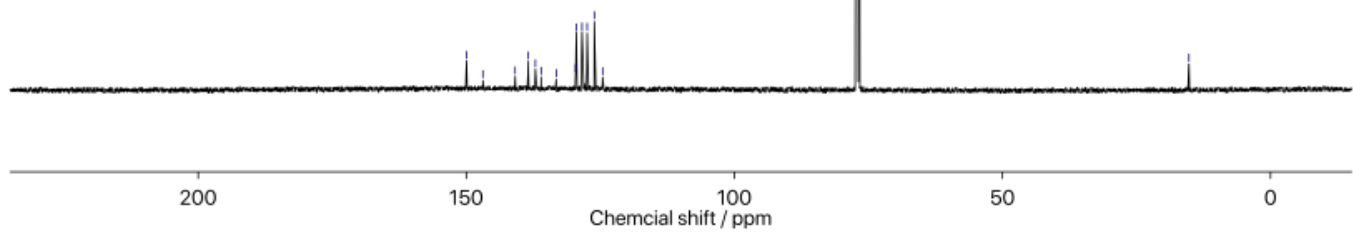

Figure S24. ${ }^{13} \mathrm{C}$ NMR spectrum of $\mathbf{P 7}\left(125 \mathrm{MHz}, \mathrm{CDCl}_{3}\right)$. 


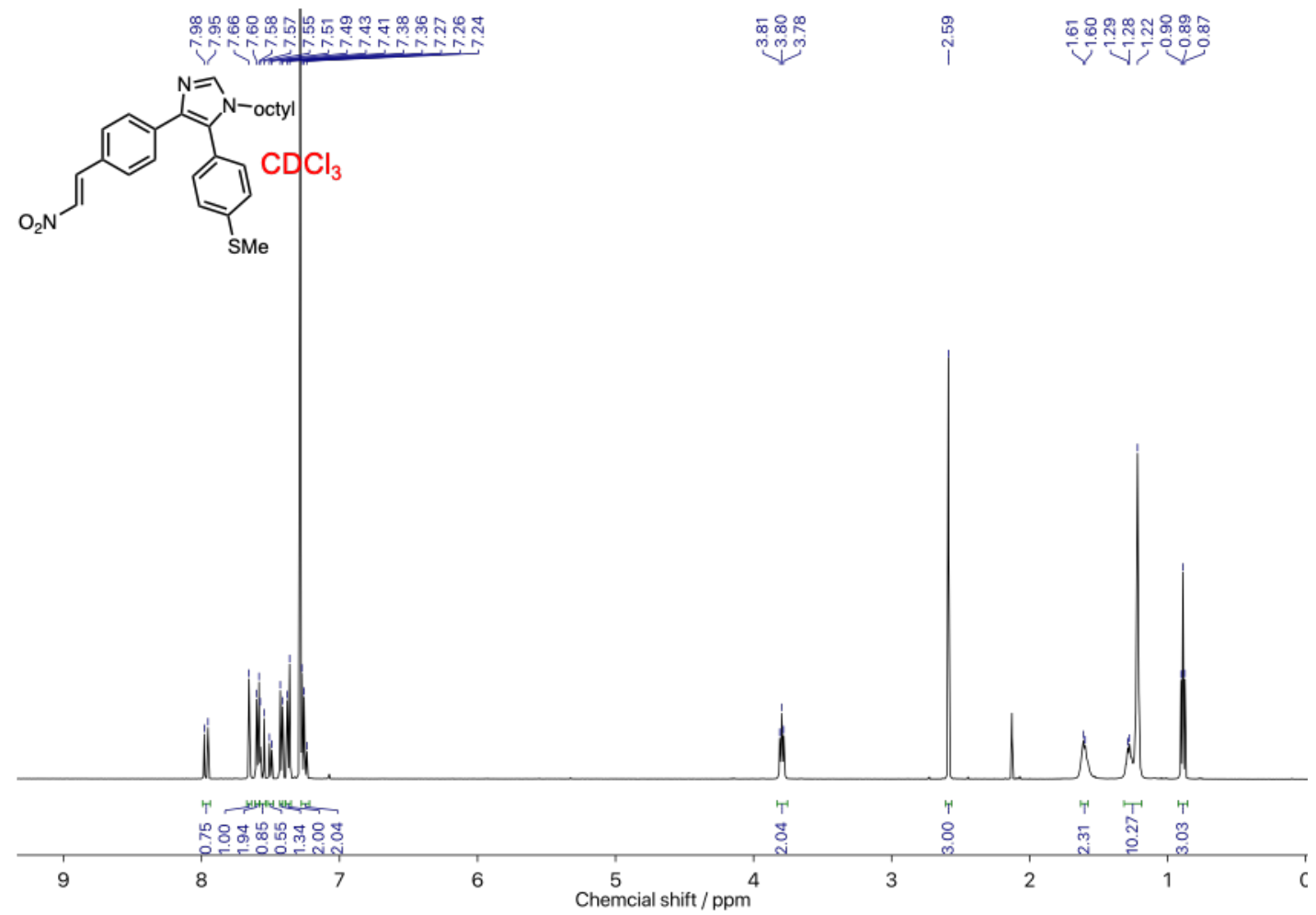

Figure S25. ${ }^{1} \mathrm{H}$ NMR spectrum of $\left.\mathbf{P 8}\left(500 \mathrm{MHz}^{\mathrm{CDCl}}\right)_{3}\right)$.
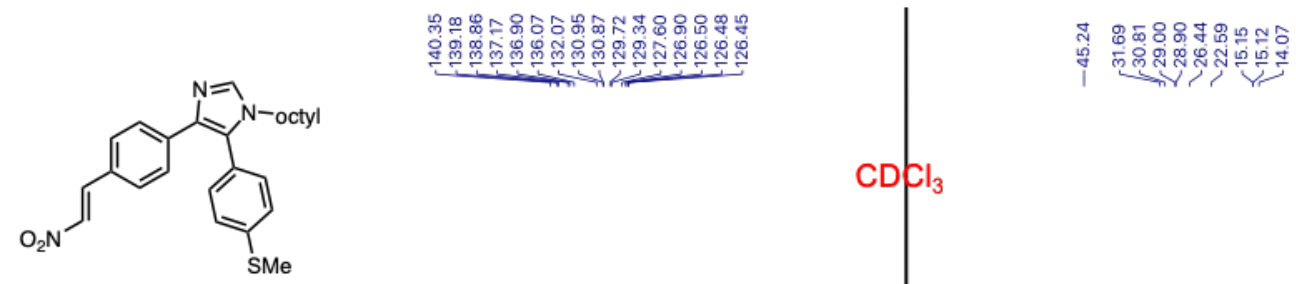

(iii)
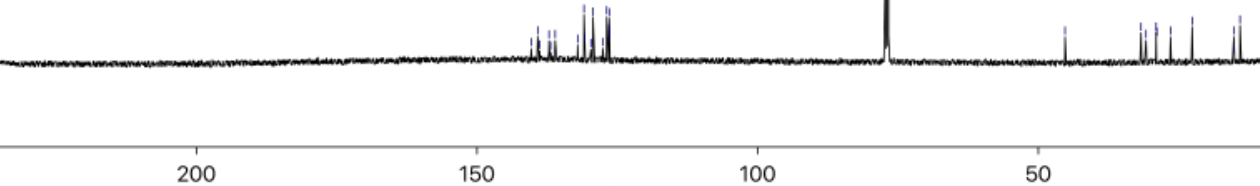

$200 \quad 150$ Chemcial shift/ppm

50

0

Figure S26. ${ }^{13} \mathrm{C}$ NMR spectrum of $\mathbf{P 8}\left(125 \mathrm{MHz}, \mathrm{CDCl}_{3}\right)$. 


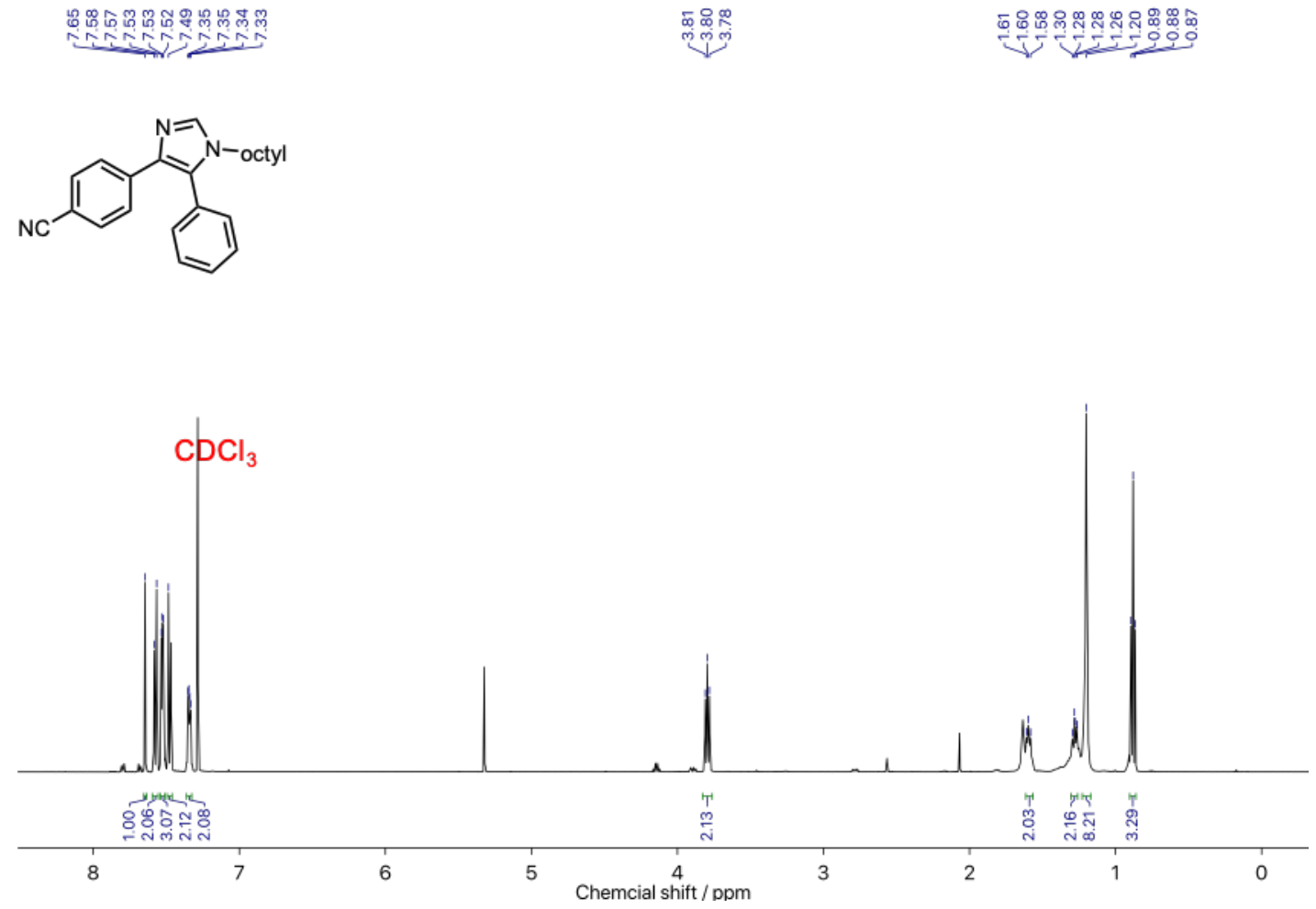

Figure S27. ${ }^{1} \mathrm{H}$ NMR spectrum of $\mathbf{P 9}\left(500 \mathrm{MHz}, \mathrm{CDCl}_{3}\right)$.

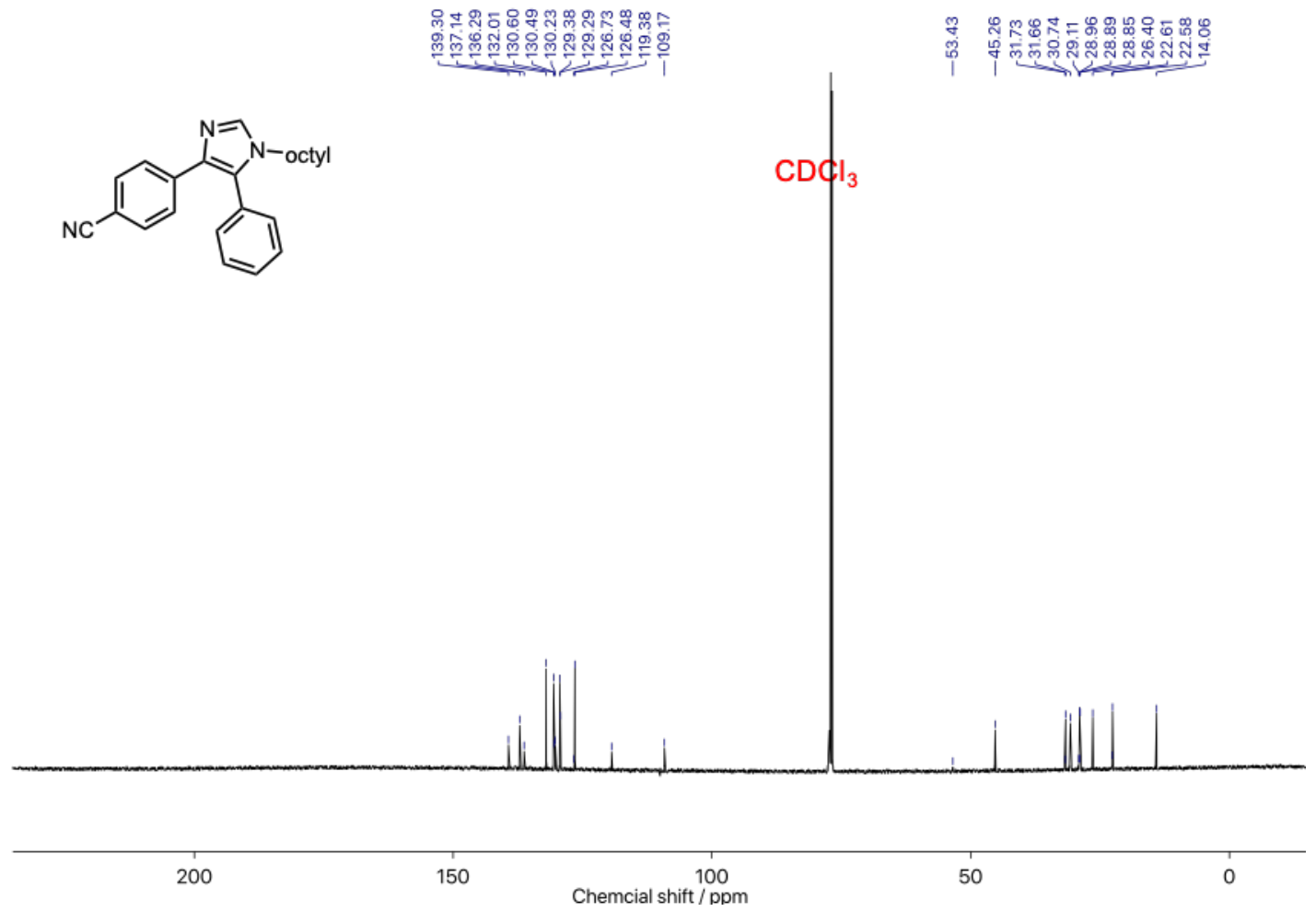

Figure S28. ${ }^{13} \mathrm{C}$ NMR spectrum of $\mathbf{P 9}\left(125 \mathrm{MHz}, \mathrm{CDCl}_{3}\right)$. 


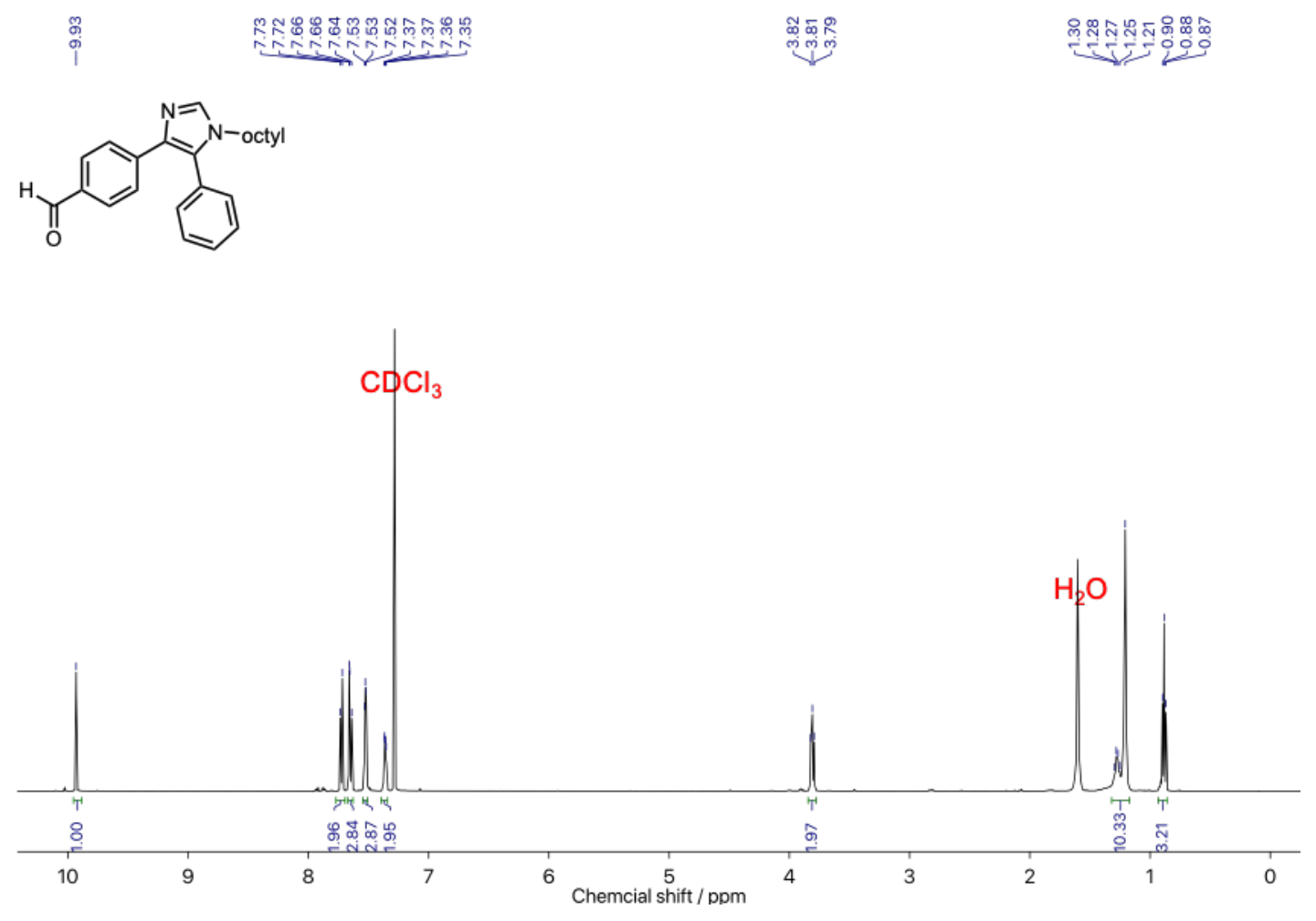

Figure S29. ${ }^{1} \mathrm{H}$ NMR spectrum of $\mathbf{P 1 0}\left(500 \mathrm{MHz}, \mathrm{CDCl}_{3}\right)$.

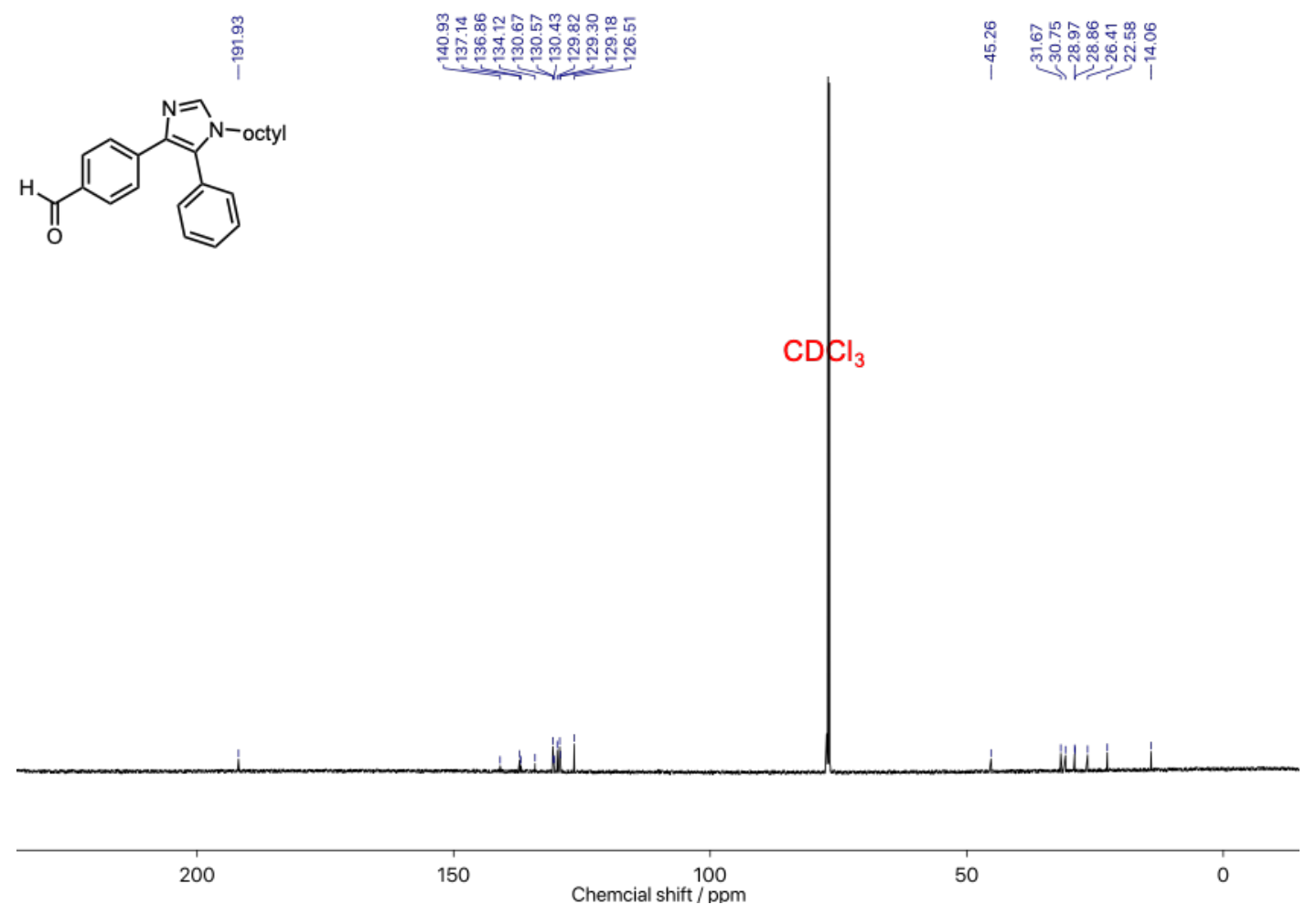

Figure S30. ${ }^{13} \mathrm{C} \mathrm{NMR}$ spectrum of $\mathbf{P 1 0}\left(125 \mathrm{MHz}, \mathrm{CDCl}_{3}\right)$. 


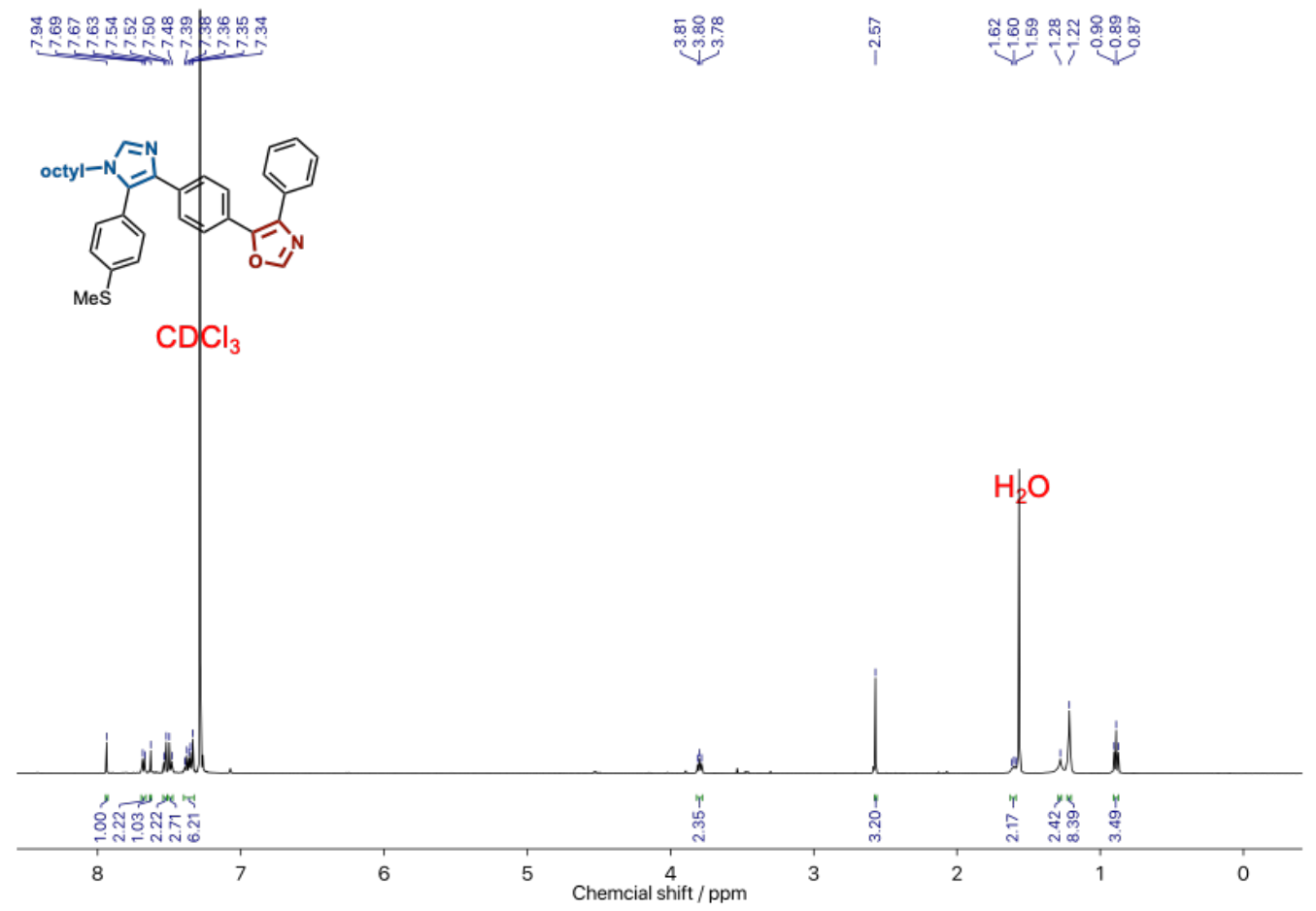

Figure S31. ${ }^{1} \mathrm{H}$ NMR spectrum of S-PIPOP-H $\left(500 \mathrm{MHz}, \mathrm{CDCl}_{3}\right)$.

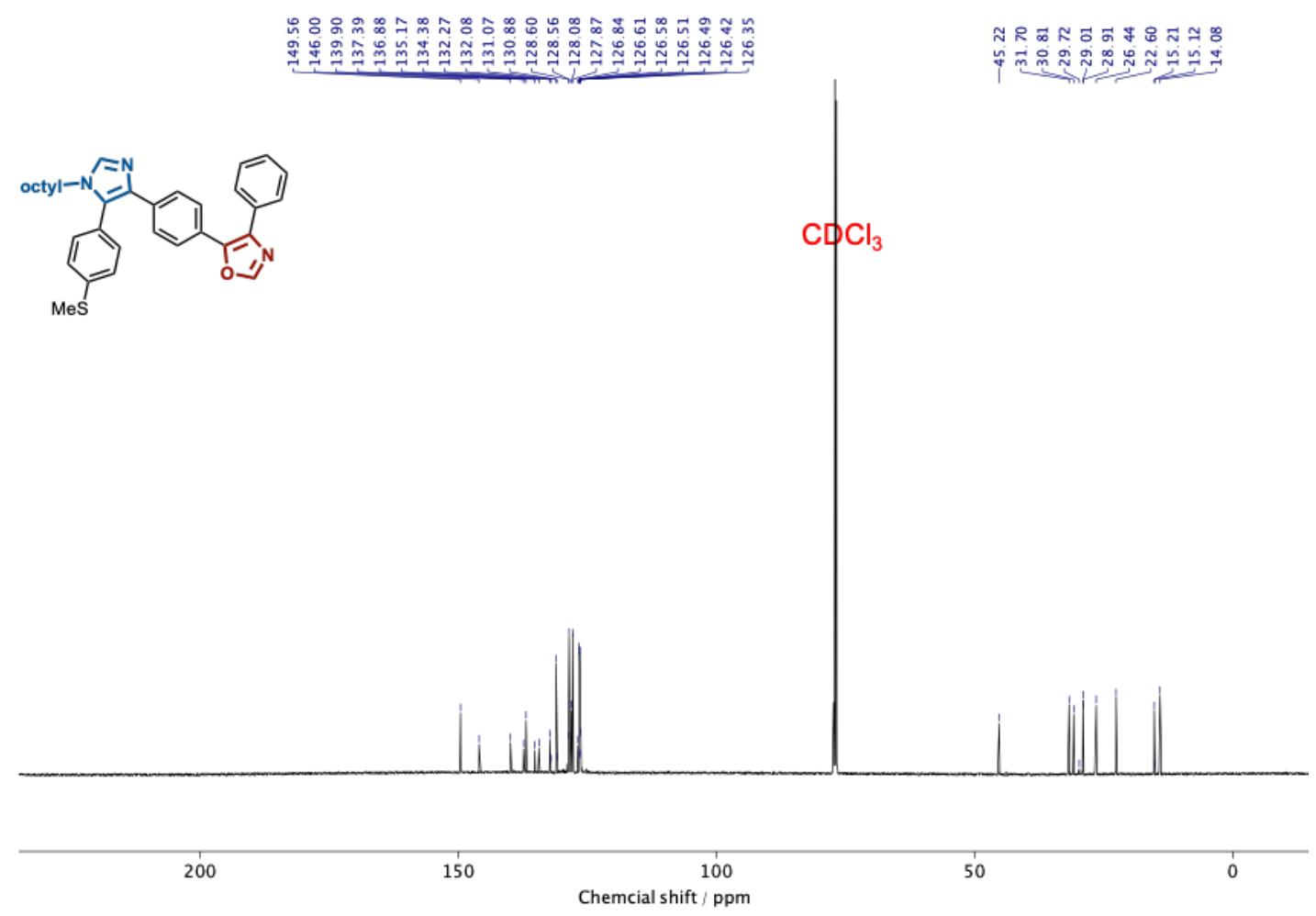

Figure S32. ${ }^{13} \mathrm{C}$ NMR spectrum of S-PIPOP-H (125 MHz, $\left.\mathrm{CDCl}_{3}\right)$. 


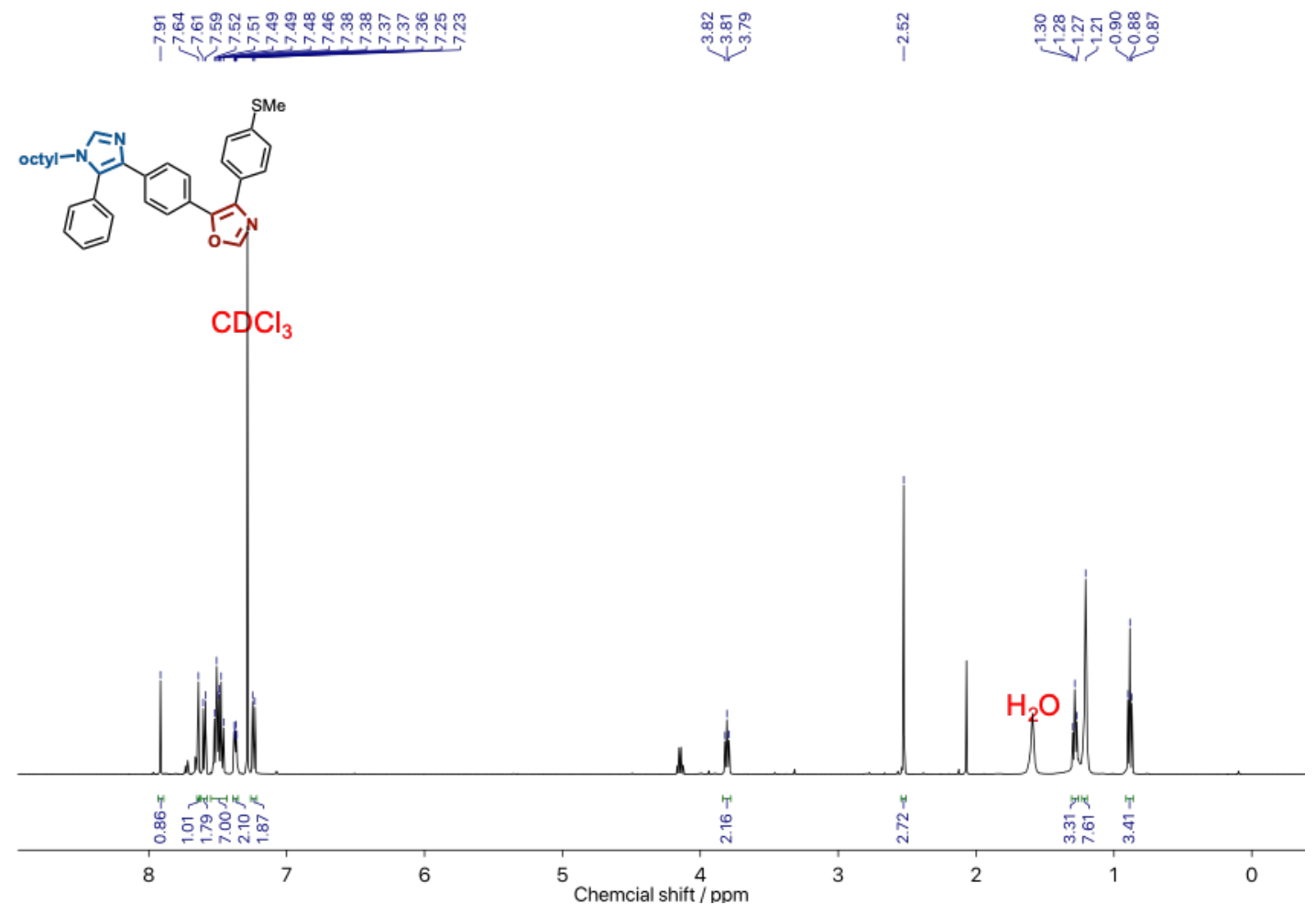

Figure S33. ${ }^{1} \mathrm{H}$ NMR spectrum of H-PIPOP-S (500 MHz, $\left.\mathrm{CDCl}_{3}\right)$.

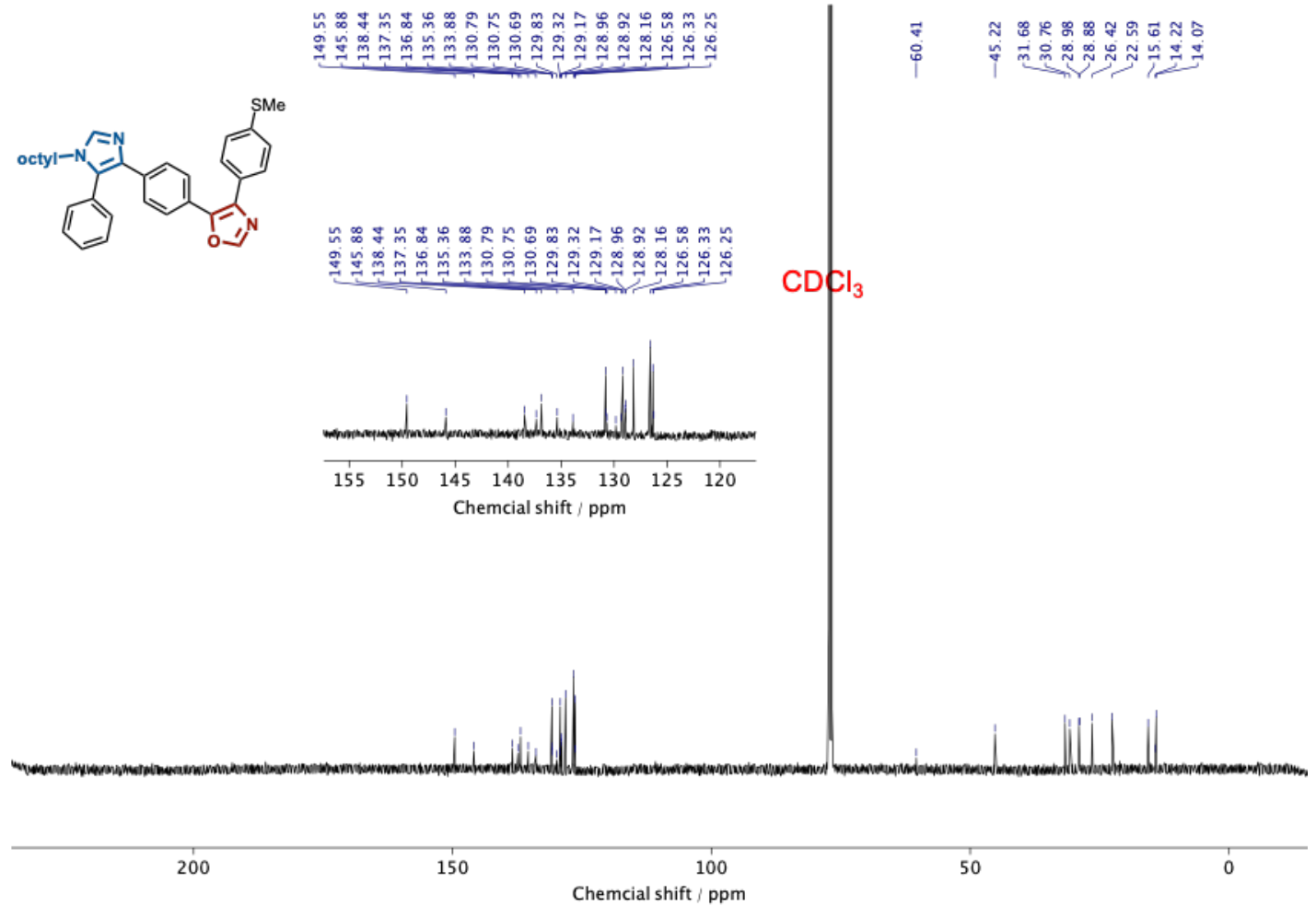

Figure S34. ${ }^{13} \mathrm{C}$ NMR spectrum of H-PIPOP-S (125 MHz, $\left.\mathrm{CDCl}_{3}\right)$. 


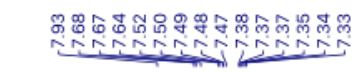

.<smiles></smiles>
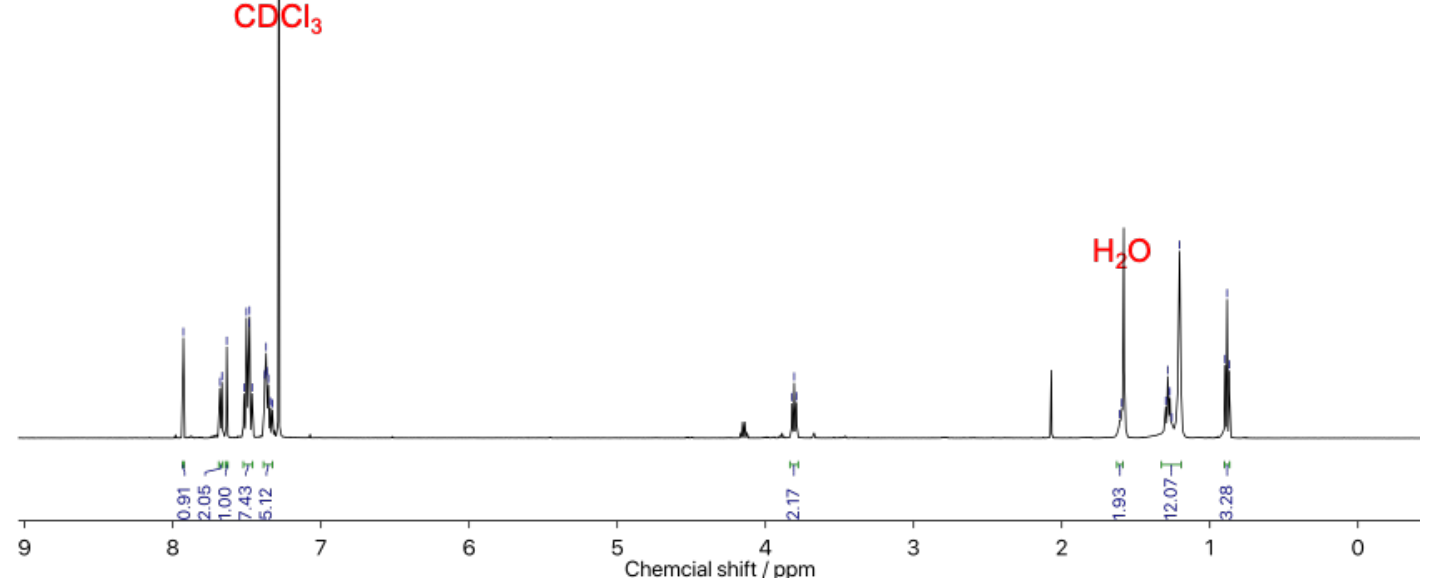

Figure S35. ${ }^{1} \mathrm{H}$ NMR spectrum of H-PIPOP-H (500 MHz, $\left.\mathrm{CDCl}_{3}\right)$.

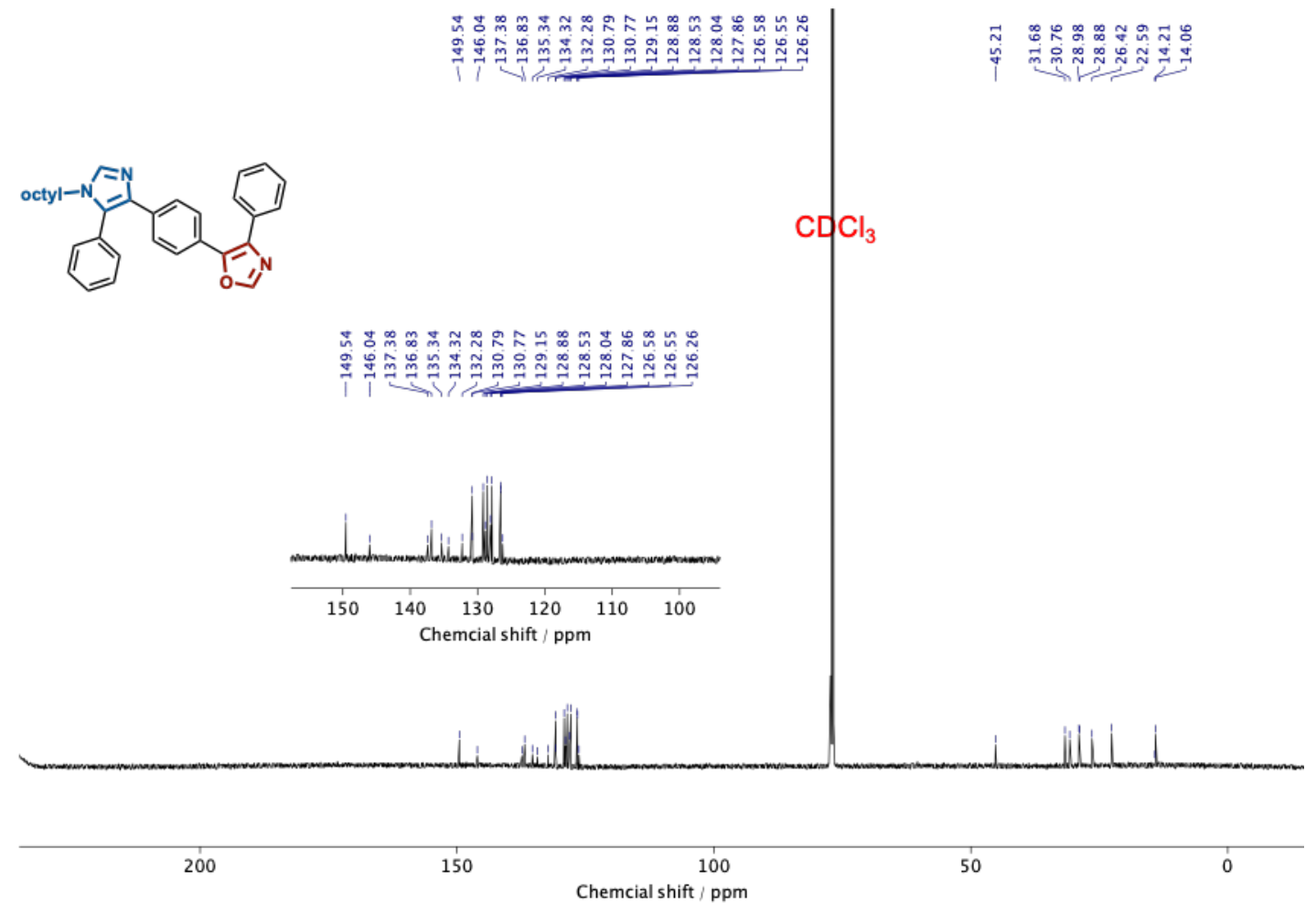

Figure S36. ${ }^{13} \mathrm{C}$ NMR spectrum of H-PIPOP-H $\left(125 \mathrm{MHz}, \mathrm{CDCl}_{3}\right)$. 


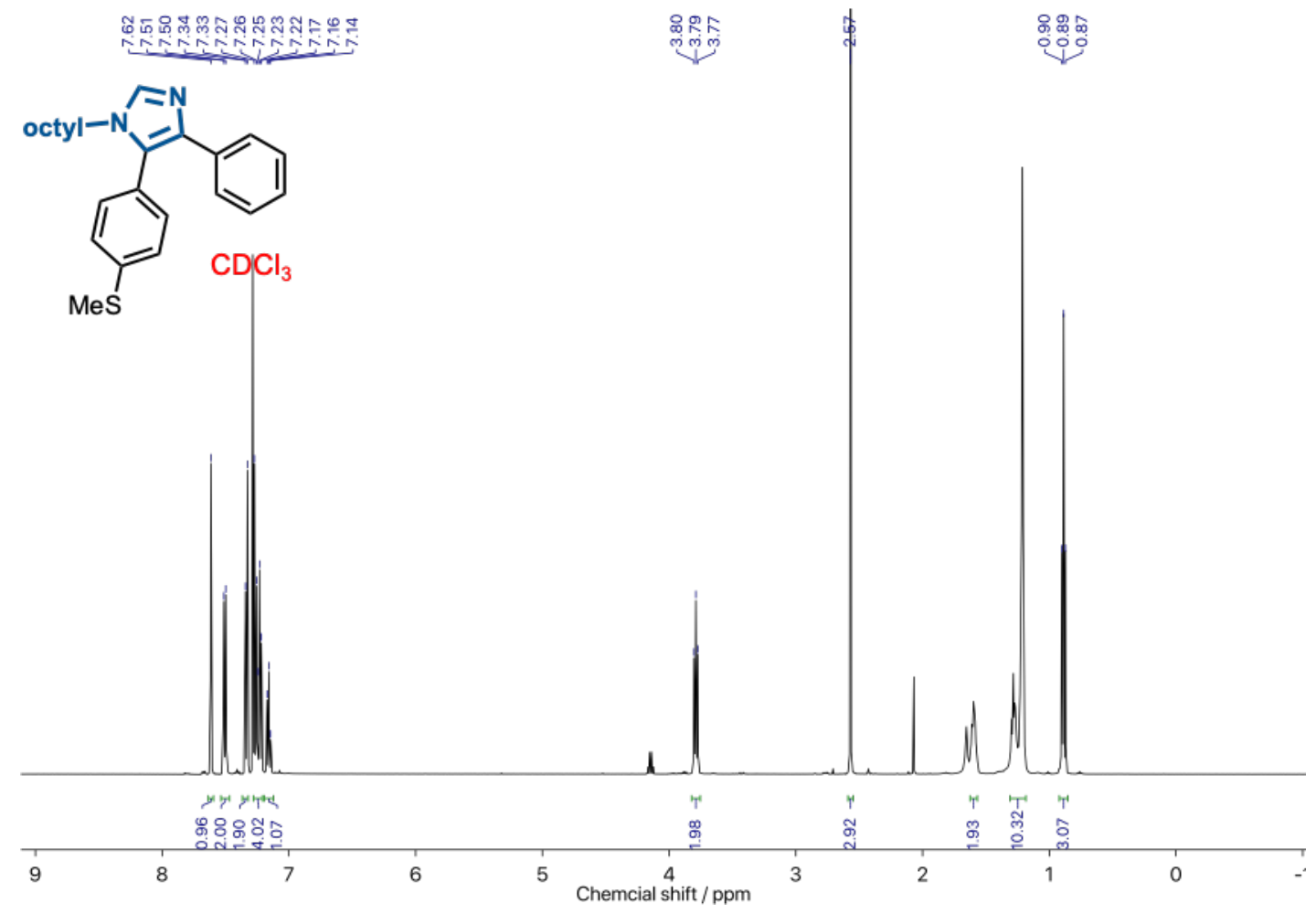

Figure S37. ${ }^{1} \mathrm{H}$ NMR spectrum of S-PIP-H $\left(500 \mathrm{MHz}, \mathrm{CDCl}_{3}\right)$.

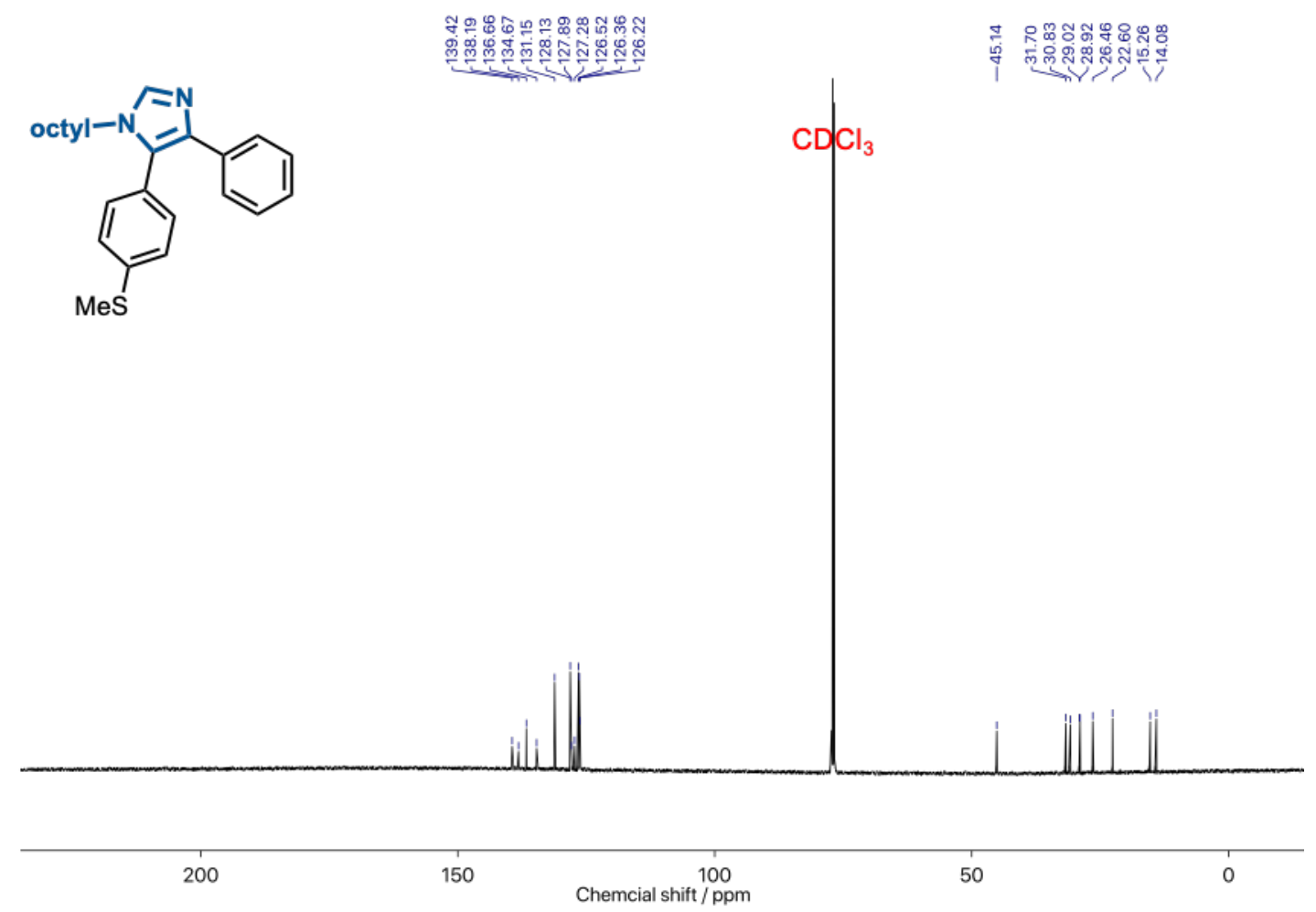

Figure S38. ${ }^{13} \mathrm{C}$ NMR spectrum of S-PIP-H (125 MHz, $\left.\mathrm{CDCl}_{3}\right)$. 

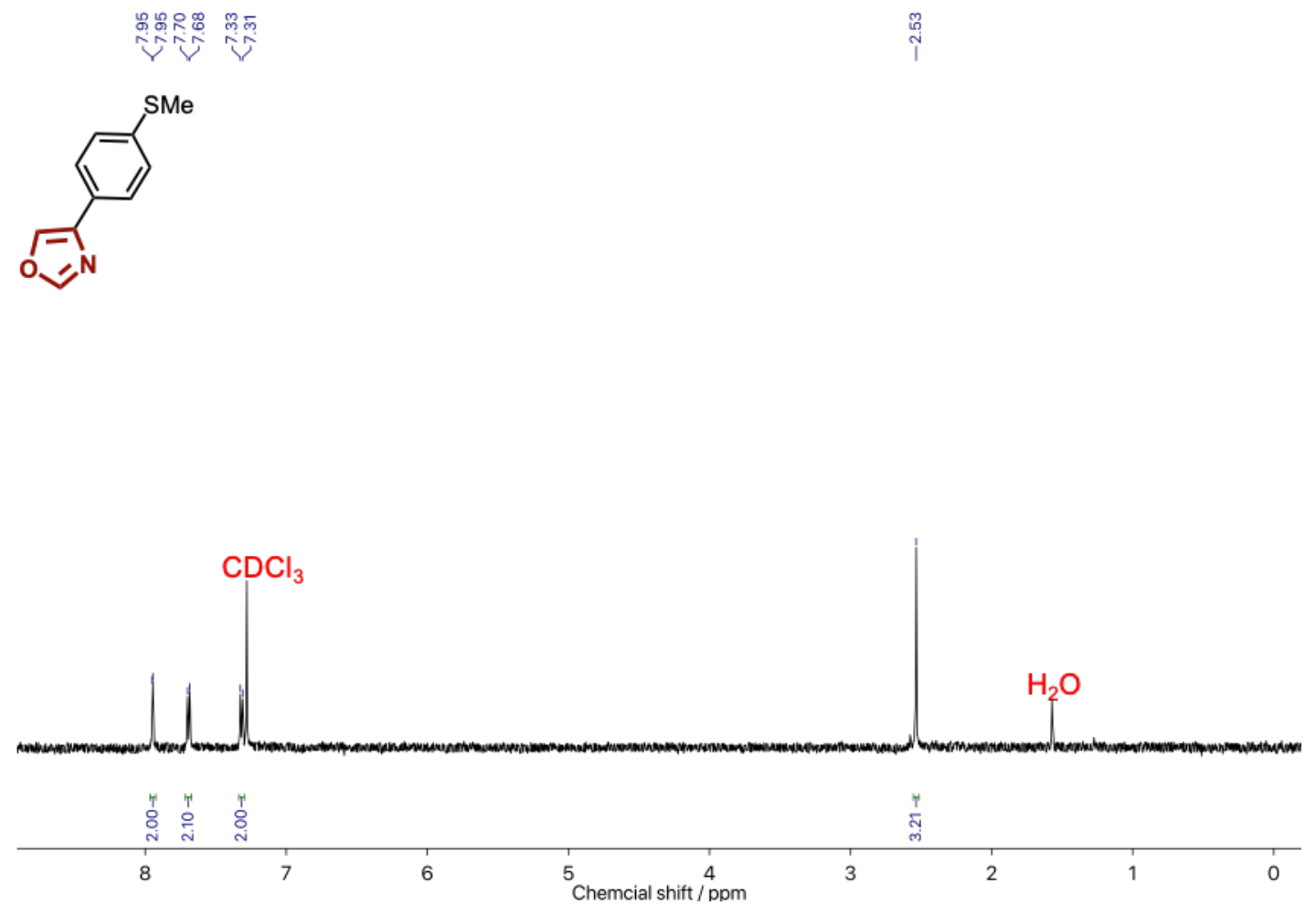

Figure S39. ${ }^{1} \mathrm{H}$ NMR spectrum of H-OP-S $\left(500 \mathrm{MHz}, \mathrm{CDCl}_{3}\right)$.

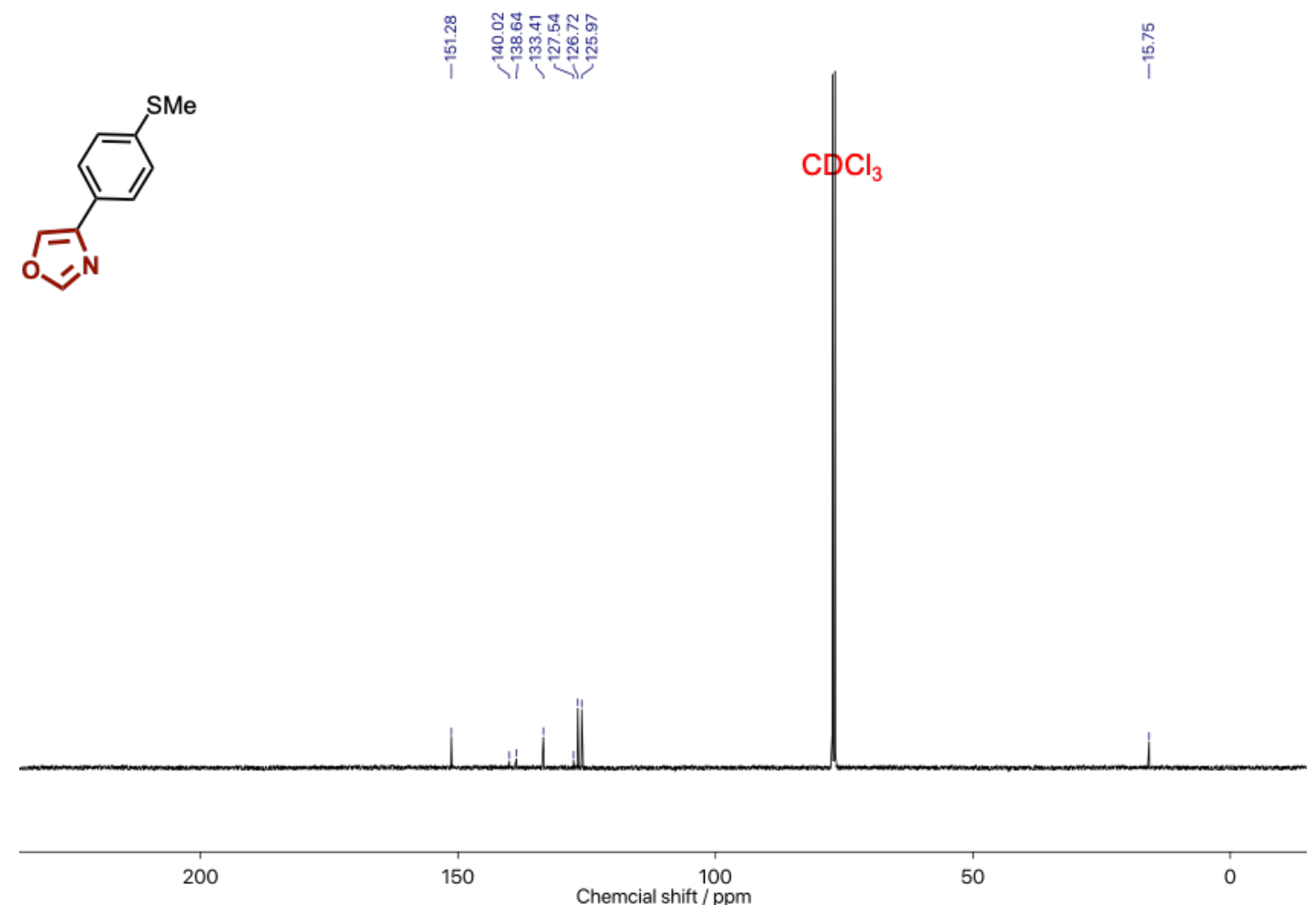

Figure S40. ${ }^{13} \mathrm{C}$ NMR spectrum of $\mathbf{H - O P - S}\left(125 \mathrm{MHz}, \mathrm{CDCl}_{3}\right)$. 


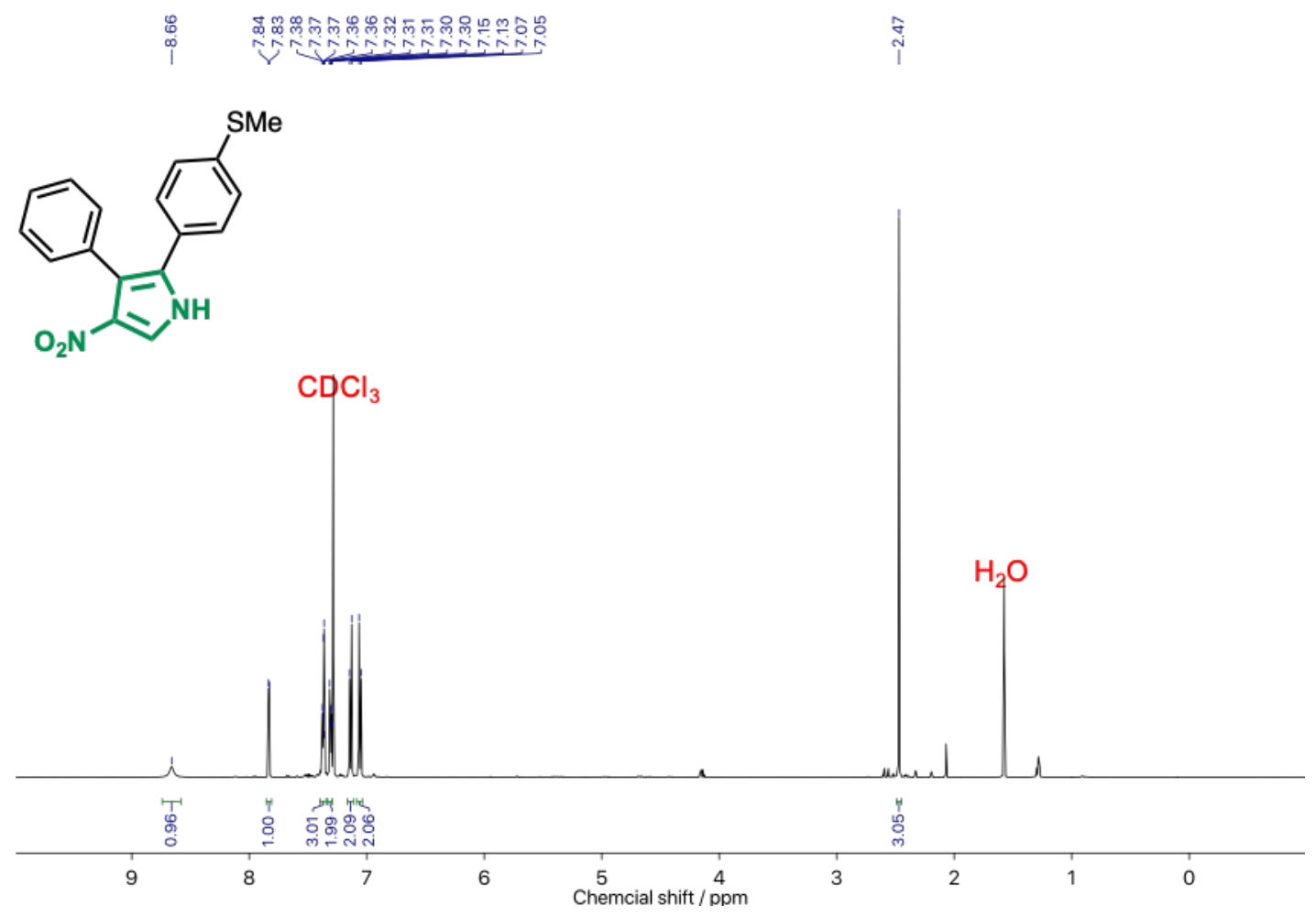

Figure S41. ${ }^{1} \mathrm{H}$ NMR spectrum of H-PRP-S (500 MHz, $\left.\mathrm{CDCl}_{3}\right)$.

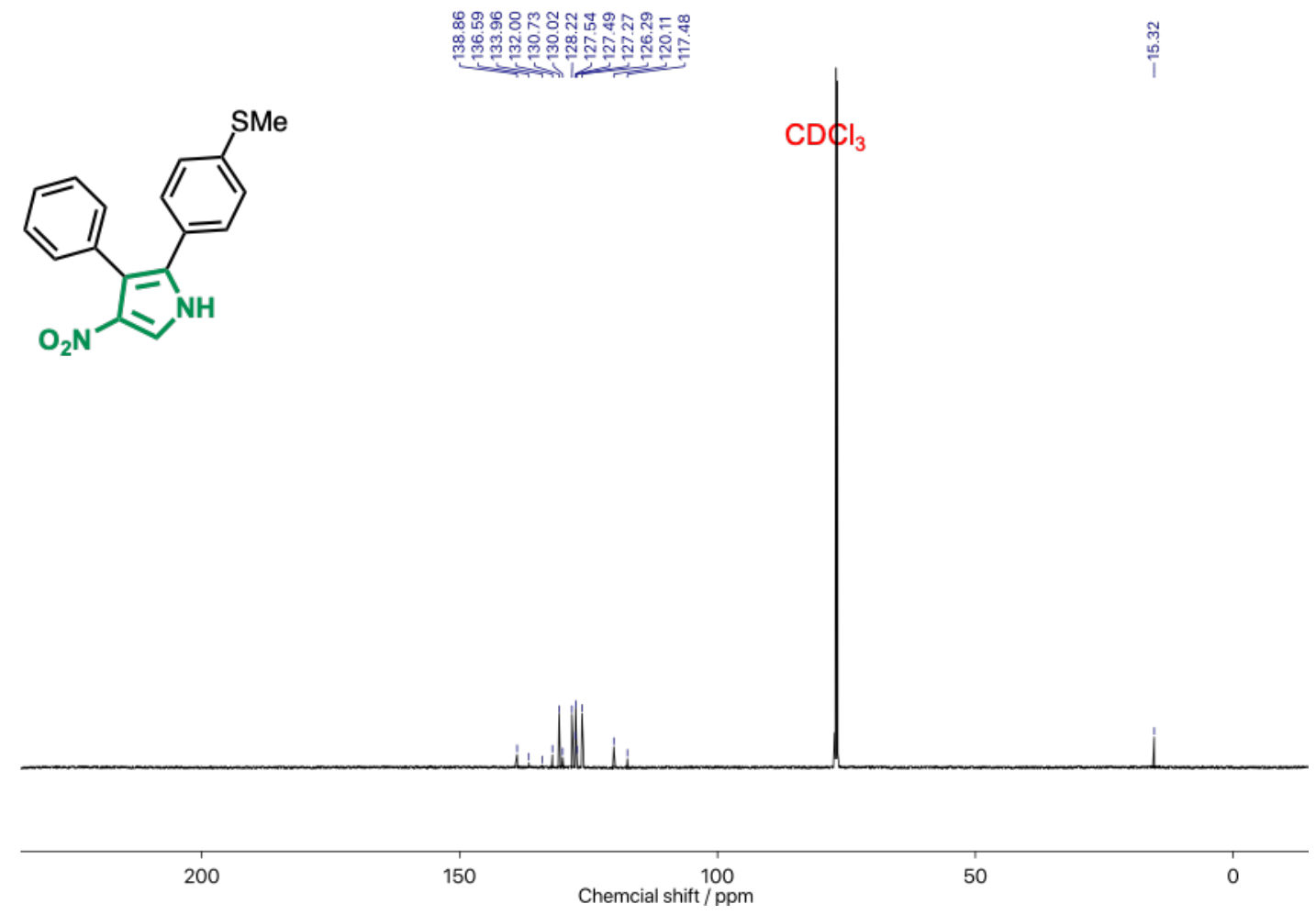

Figure S42. ${ }^{13} \mathrm{C}$ NMR spectrum of H-PRP-S (125 MHz, $\left.\mathrm{CDCl}_{3}\right)$. 


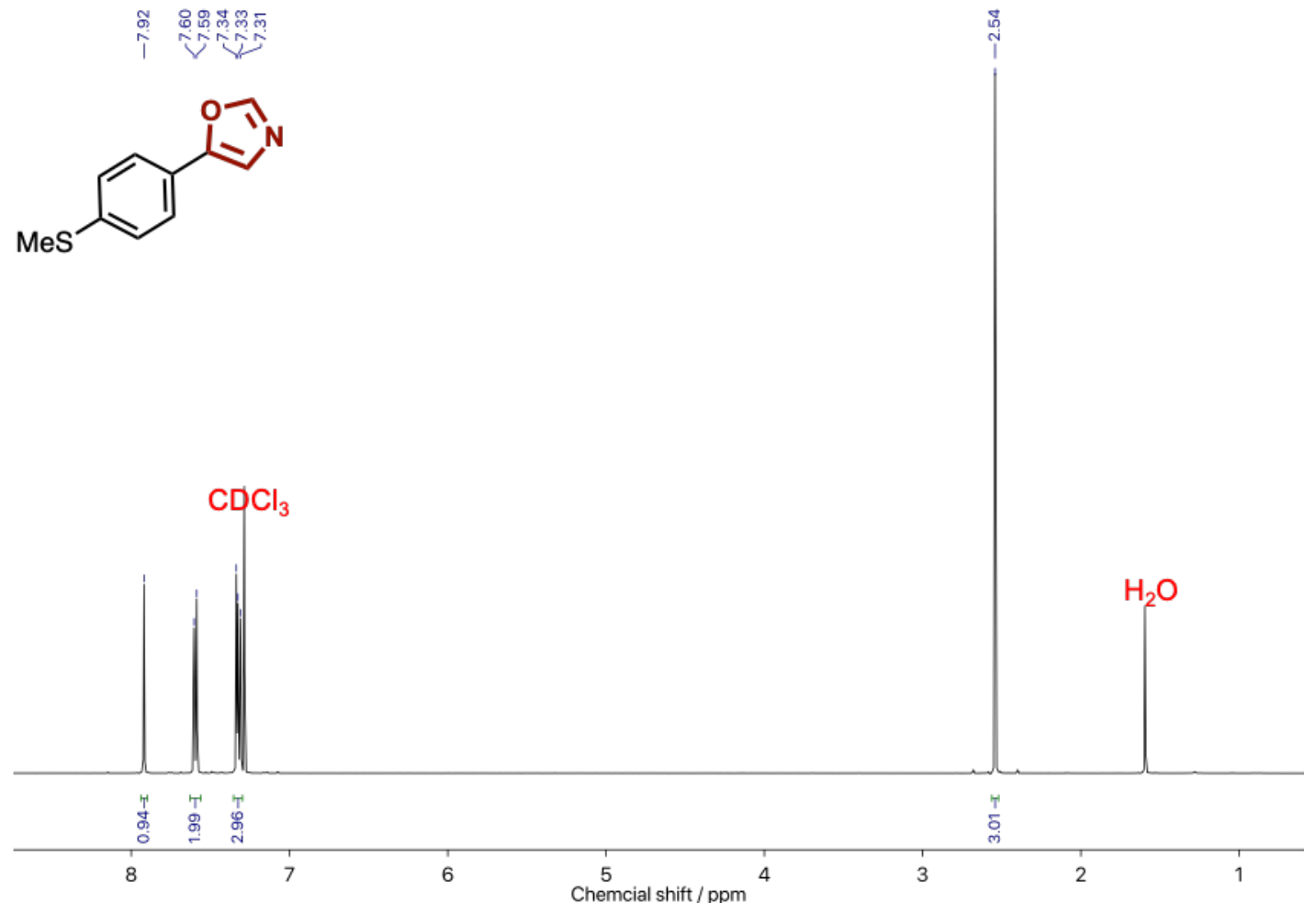

Figure S43. ${ }^{1} \mathrm{H}$ NMR spectrum of $\mathbf{P O}\left(500 \mathrm{MHz}, \mathrm{CDCl}_{3}\right)$.

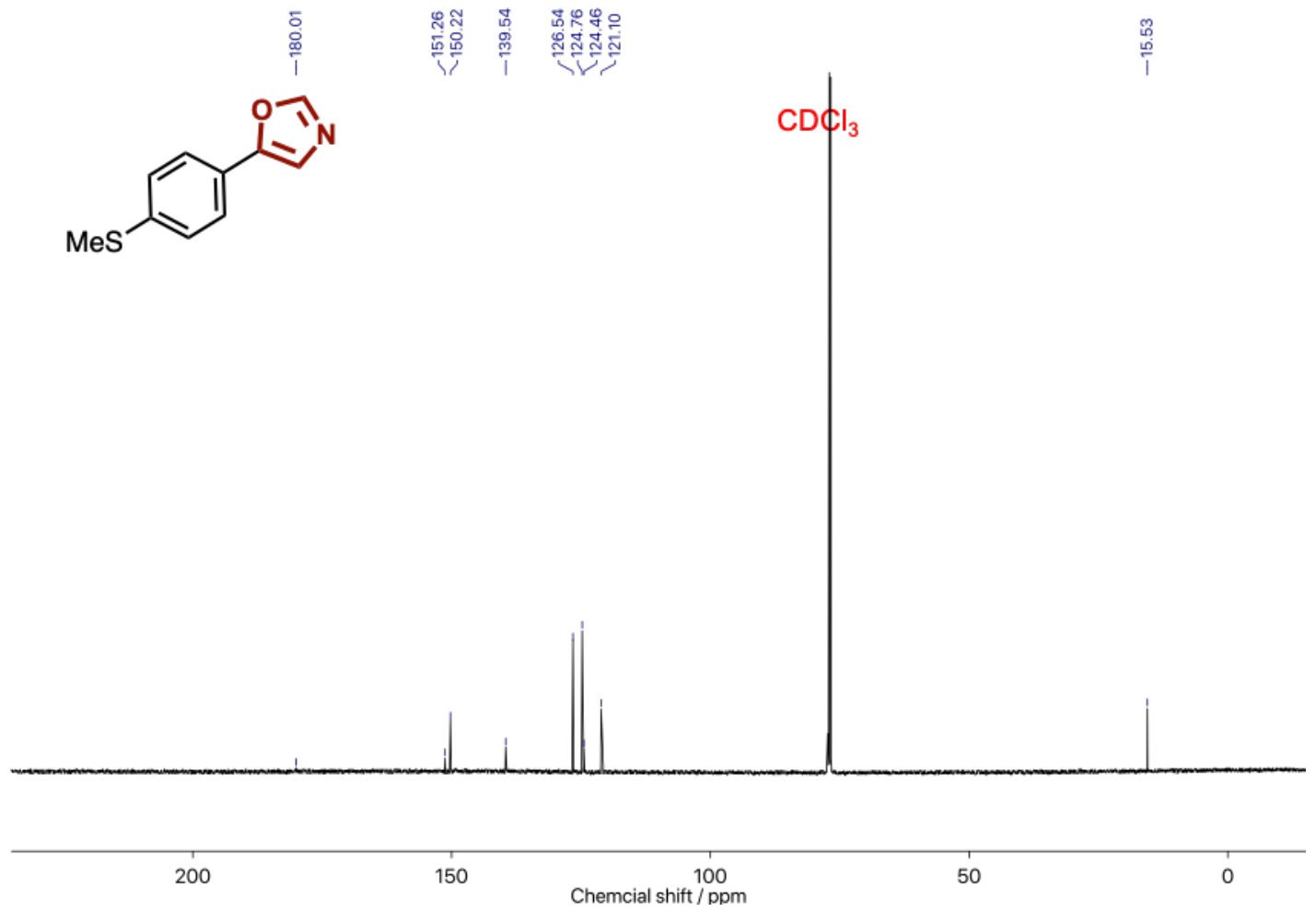

Figure S44. ${ }^{13} \mathrm{C}$ NMR spectrum of PO $\left(125 \mathrm{MHz}, \mathrm{CDCl}_{3}\right)$. 

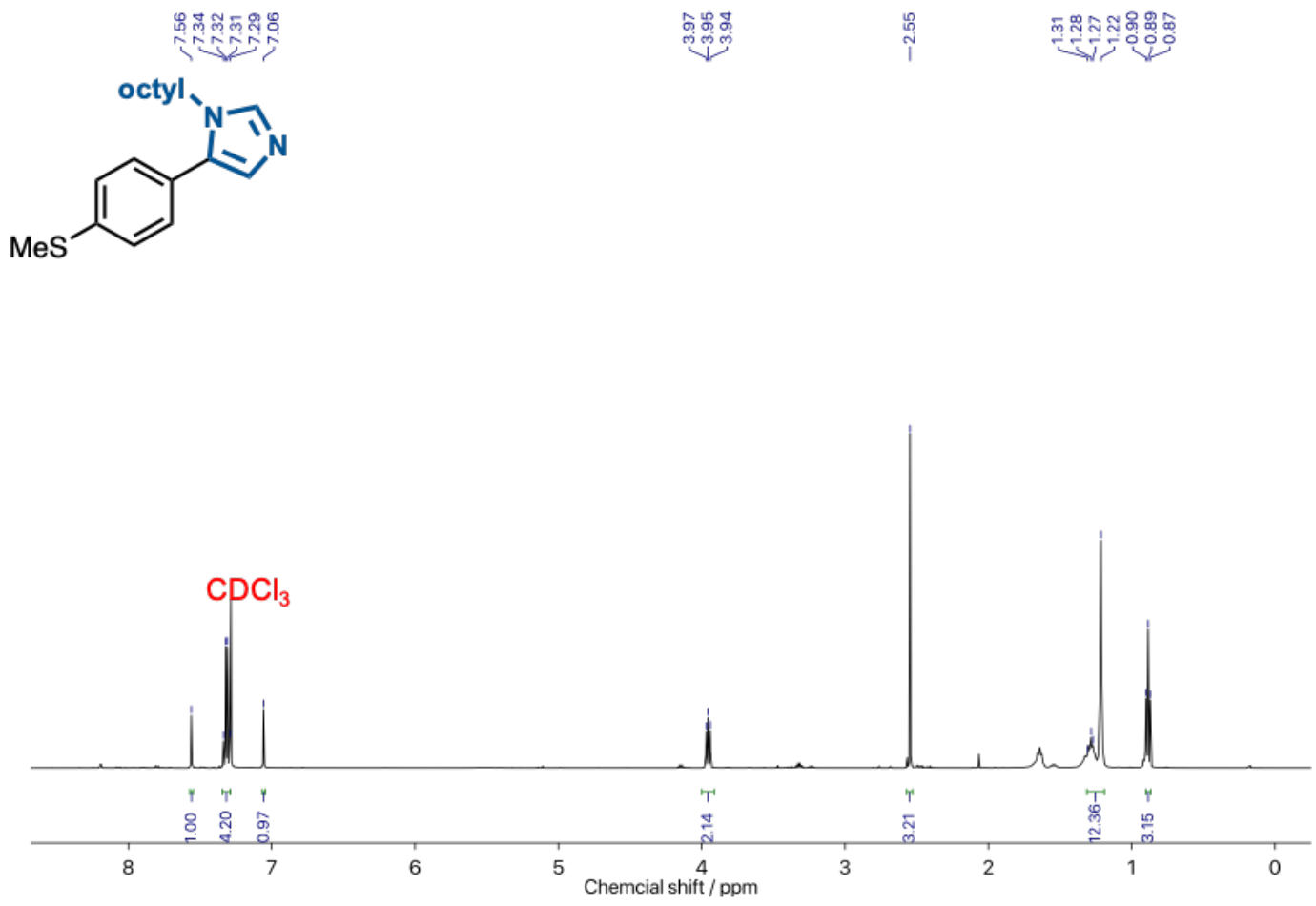

Figure S45. ${ }^{1} \mathrm{H}$ NMR spectrum of PI $\left(500 \mathrm{MHz}, \mathrm{CDCl}_{3}\right)$.

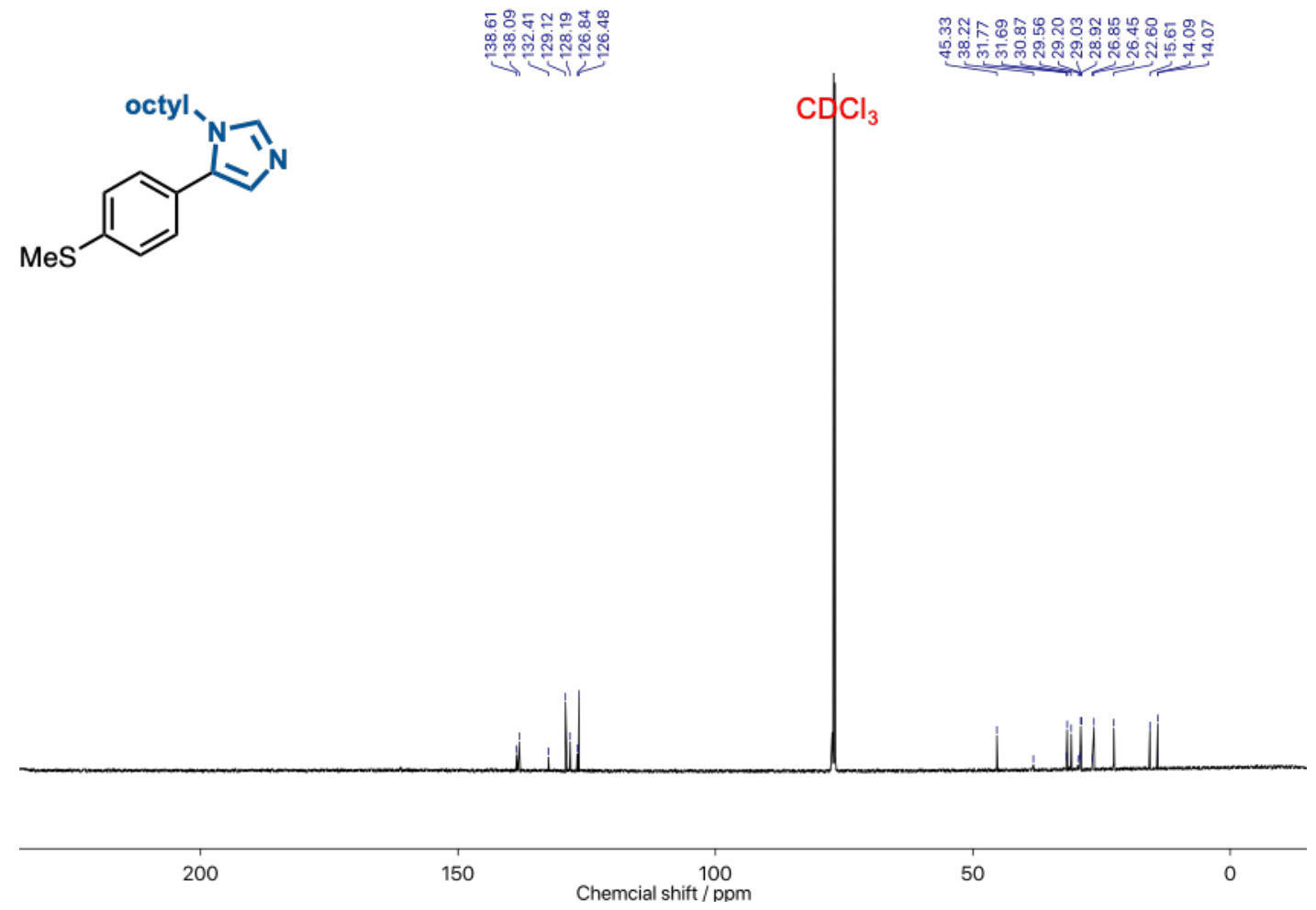

Figure S46. ${ }^{13} \mathrm{C}$ NMR spectrum of PI (125 MHz, $\left.\mathrm{CDCl}_{3}\right)$. 


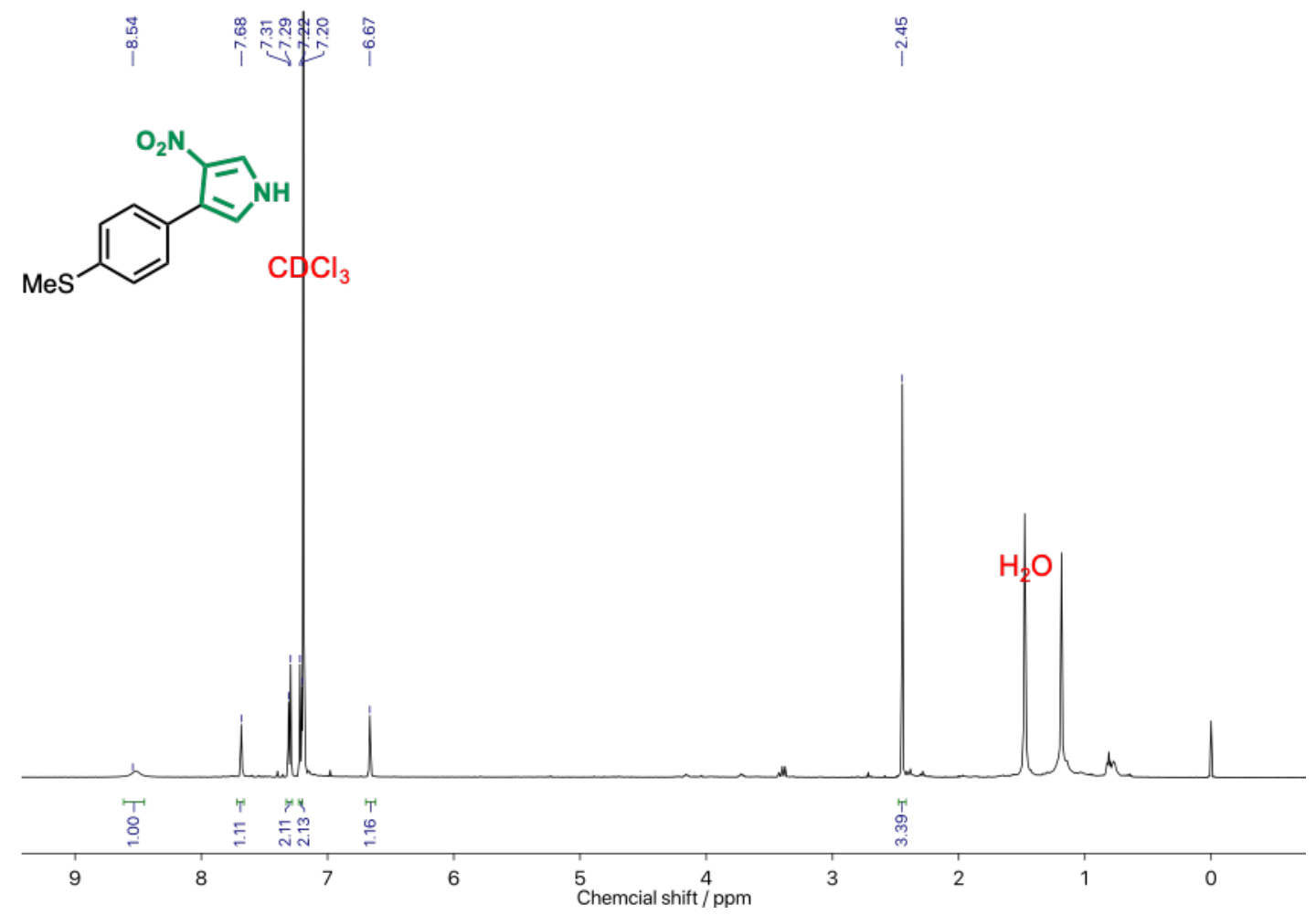

Figure S47. ${ }^{1} \mathrm{H} \mathrm{NMR}$ spectrum of $\mathbf{P R}\left(500 \mathrm{MHz}, \mathrm{CDCl}_{3}\right)$.
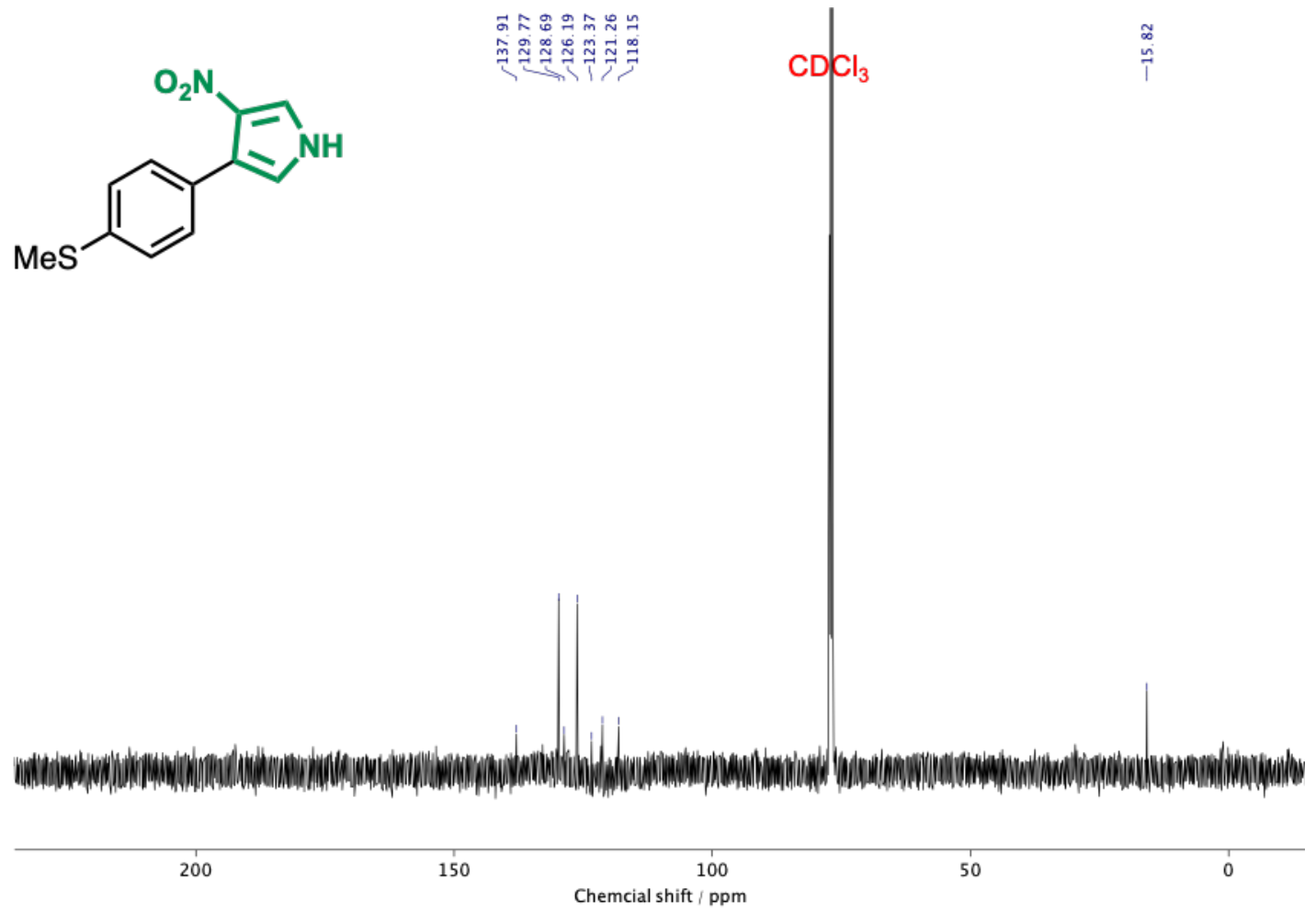

Figure S48. ${ }^{13} \mathrm{C}$ NMR spectrum of PR $\left(125 \mathrm{MHz}, \mathrm{CDCl}_{3}\right)$. 

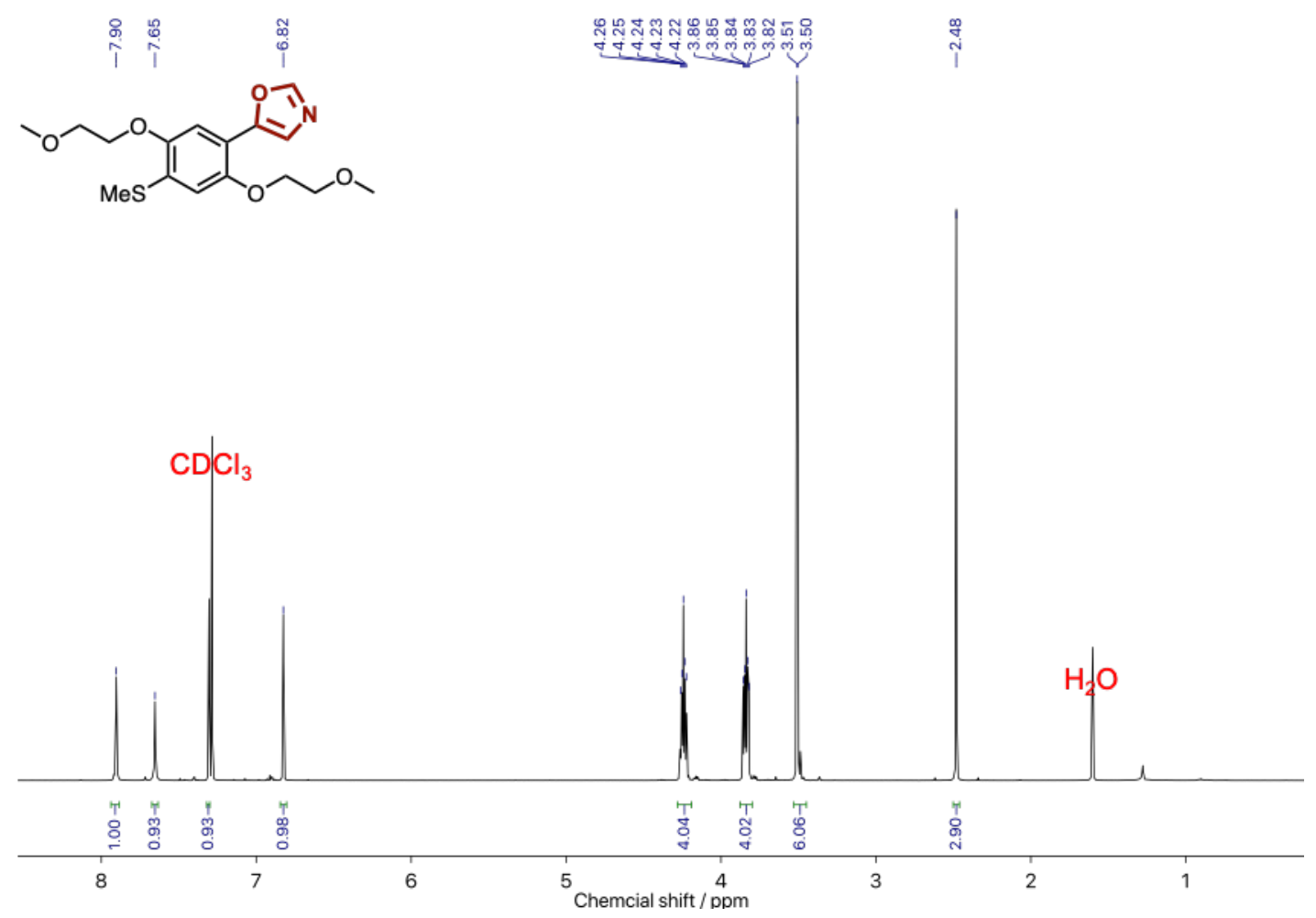

Figure S49. ${ }^{1} \mathrm{H} \mathrm{NMR}$ spectrum of $\mathbf{G O}\left(500 \mathrm{MHz}, \mathrm{CDCl}_{3}\right)$.<smiles>COCCOc1cc(S(C)(=O)=O)c(OCCOC)cc1-c1cnco1</smiles>

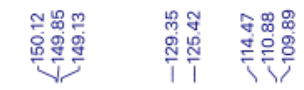
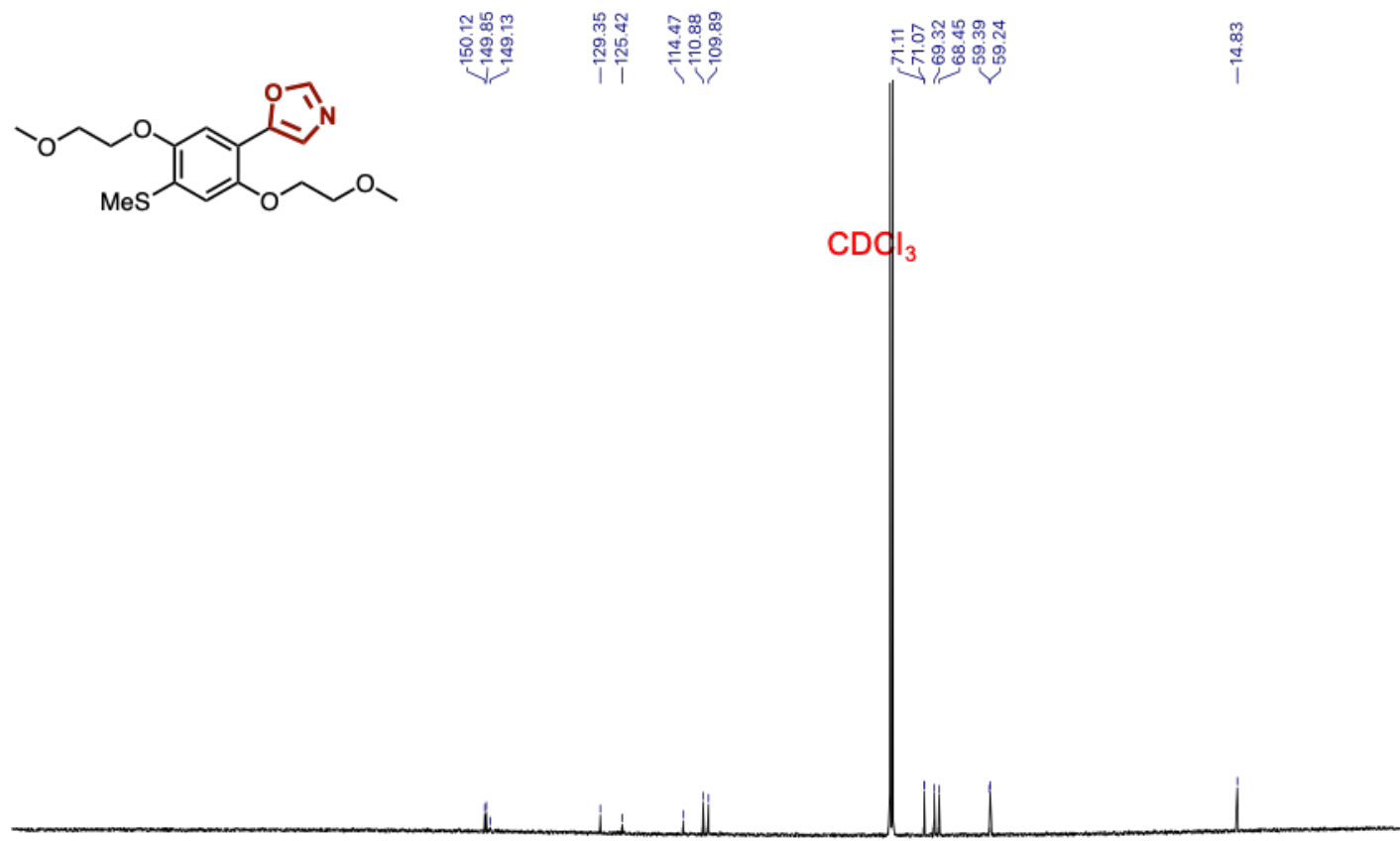

200

150

$\stackrel{100}{\text { Chemcial shift } / \mathrm{ppm}}$

50

0

Figure S50. ${ }^{13} \mathrm{C}$ NMR spectrum of GO $\left(125 \mathrm{MHz}, \mathrm{CDCl}_{3}\right)$. 


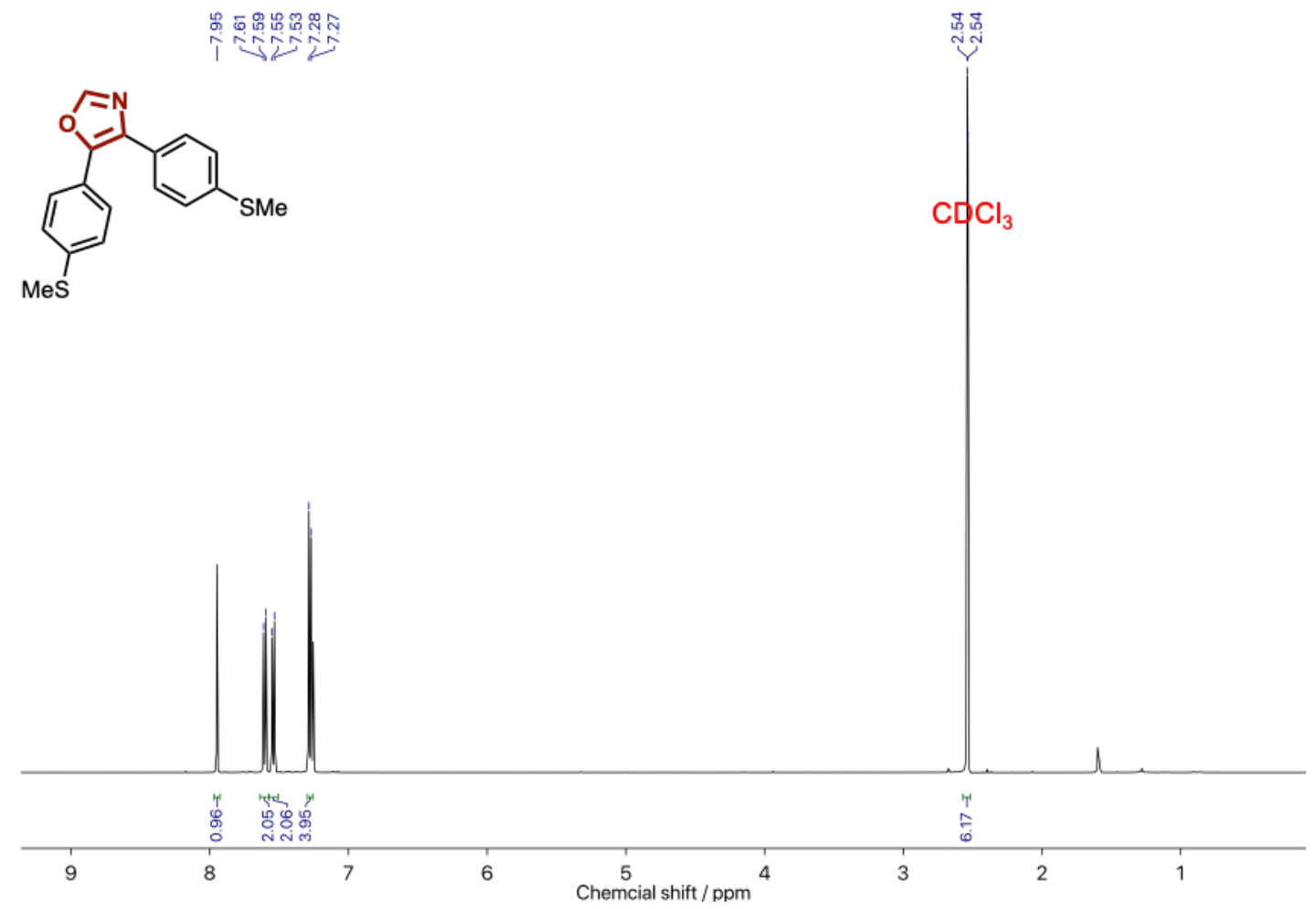

Figure S51. ${ }^{1} \mathrm{H}$ NMR spectrum of POP $\left(500 \mathrm{MHz}, \mathrm{CDCl}_{3}\right)$.

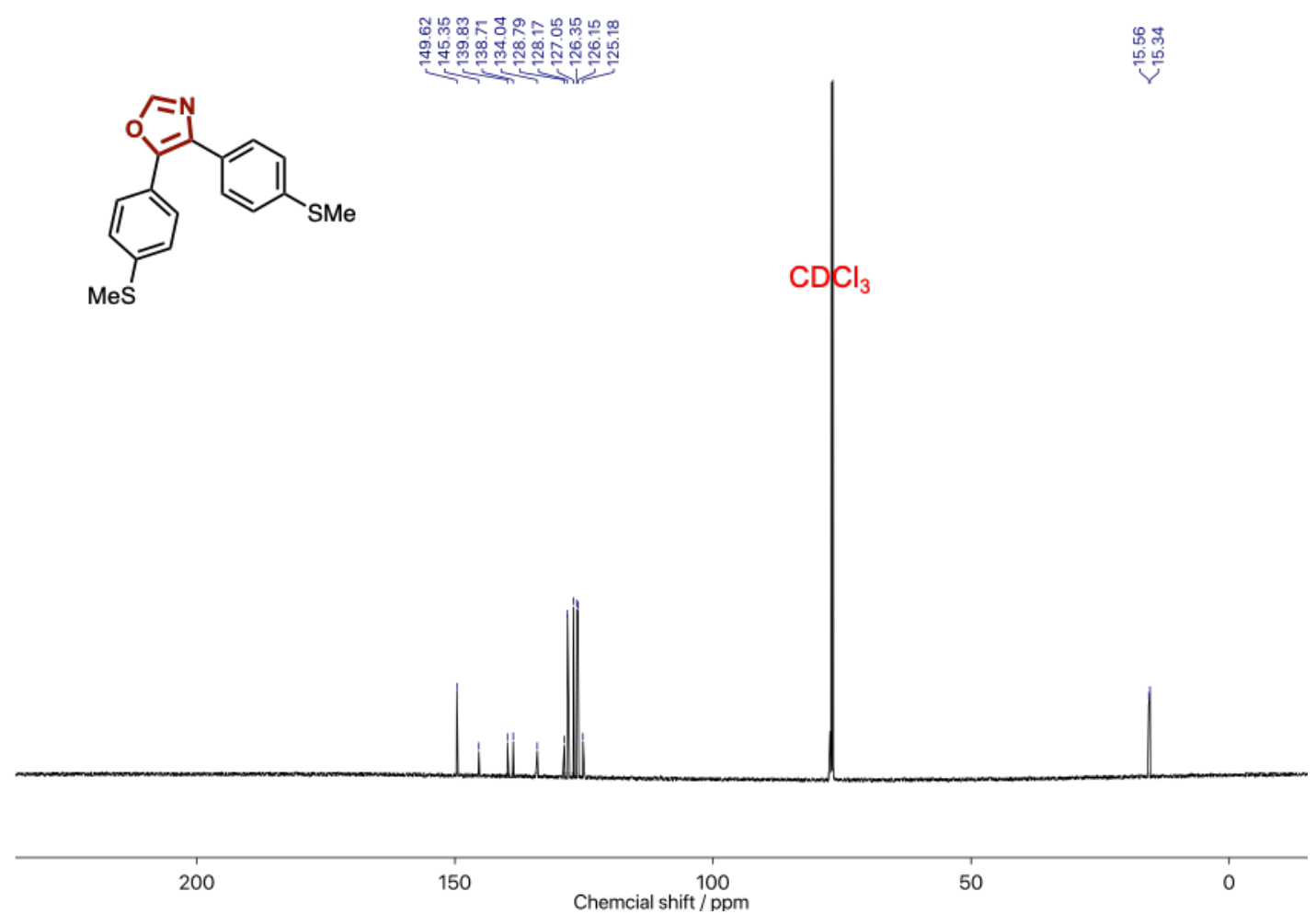

Figure S52. ${ }^{13} \mathrm{C}$ NMR spectrum of POP $\left(125 \mathrm{MHz}, \mathrm{CDCl}_{3}\right)$. 

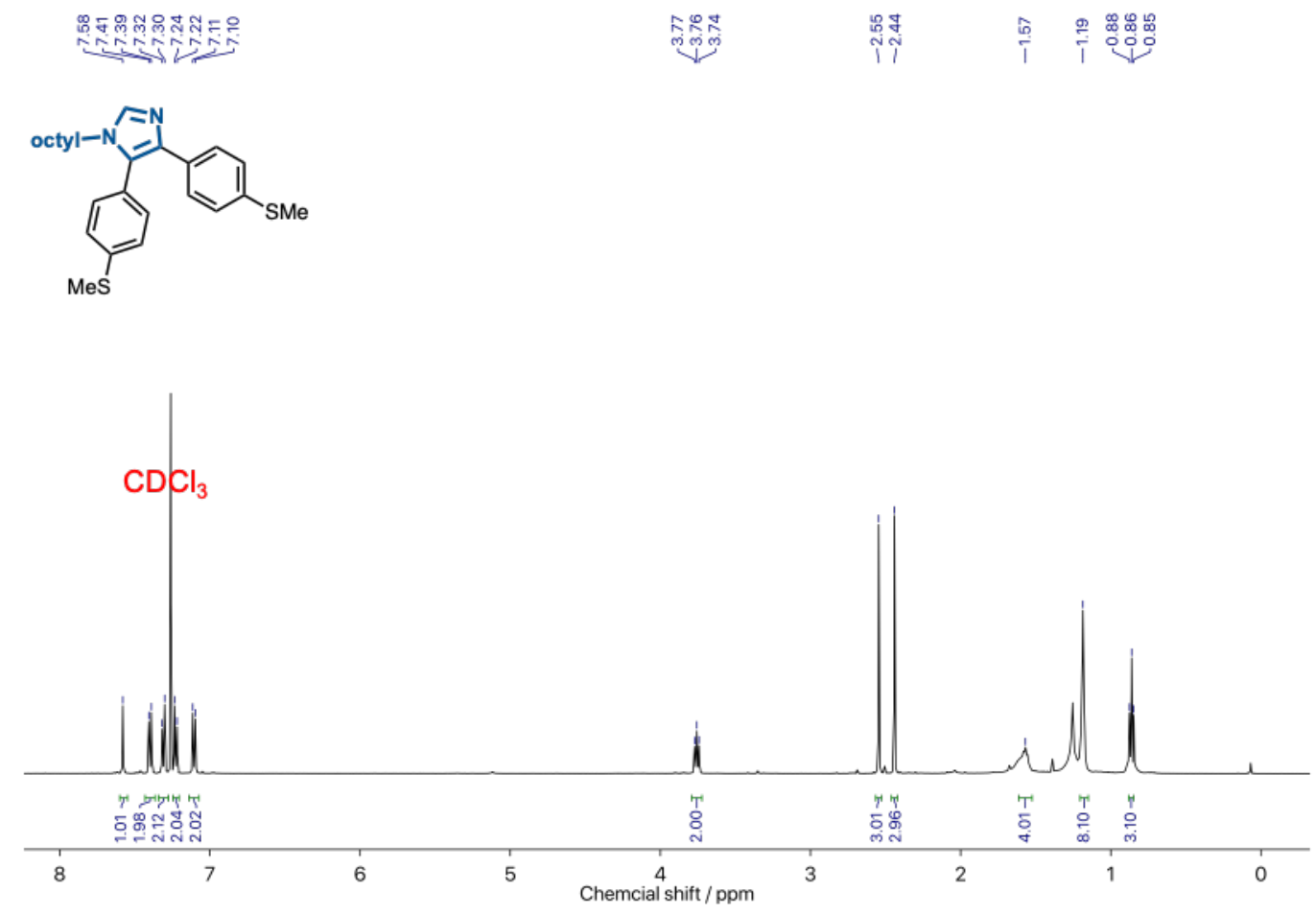

Figure S53. ${ }^{1} \mathrm{H}$ NMR spectrum of PIP $\left(500 \mathrm{MHz}, \mathrm{CDCl}_{3}\right)$.<smiles>COc1ccc(-c2ncn([C+]([O-])O[Na])c2-c2ccc(S(C)(=O)=O)cc2)cc1</smiles>

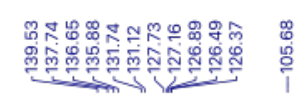

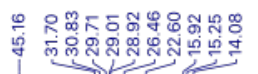
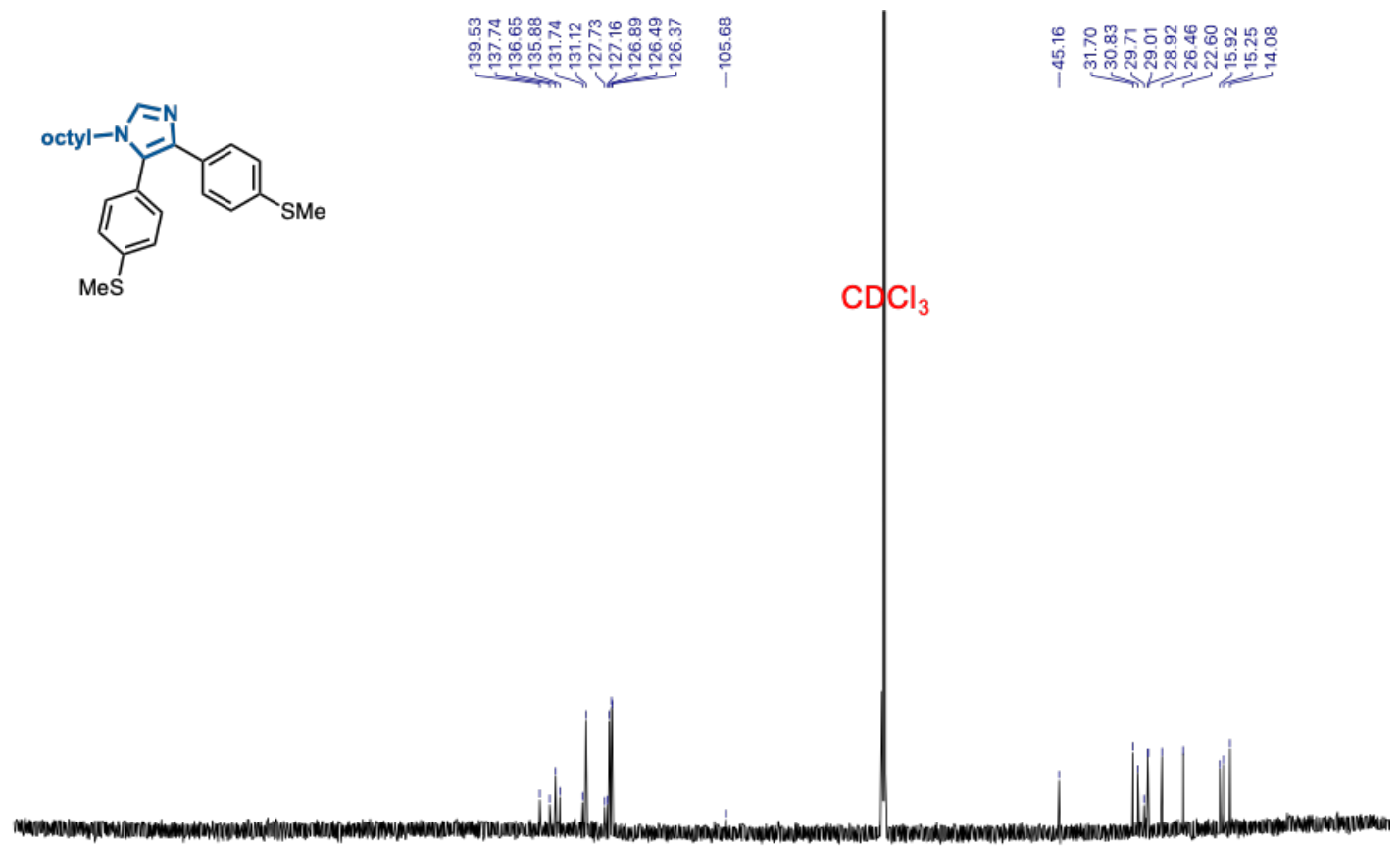

200

150

Chemcial shift $/ 100$

50

0

Figure S54. ${ }^{13} \mathrm{C}$ NMR spectrum of PIP $\left(125 \mathrm{MHz}, \mathrm{CDCl}_{3}\right)$. 


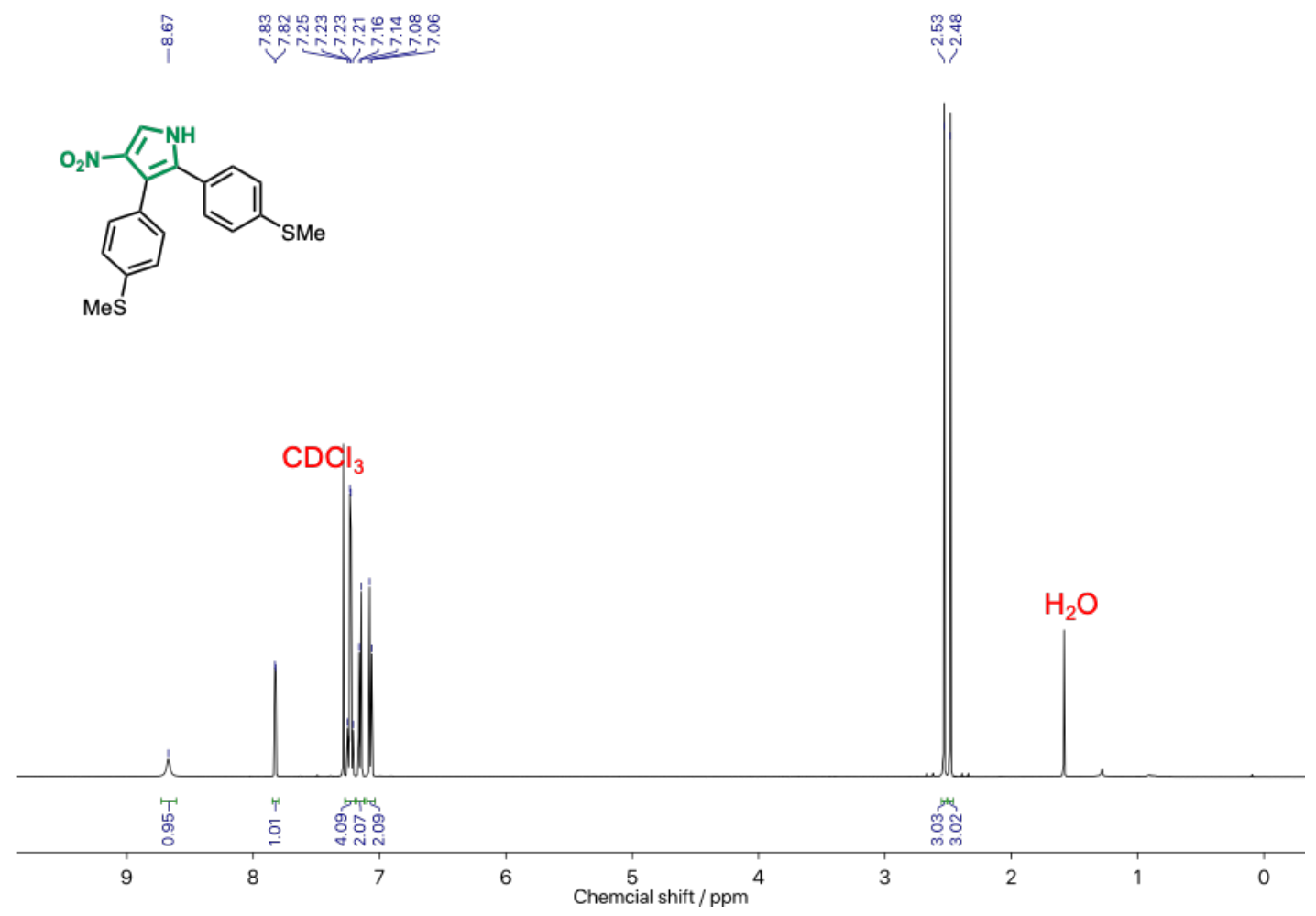

Figure S55. ${ }^{1} \mathrm{H}$ NMR spectrum of $\mathbf{P R P}\left(500 \mathrm{MHz}, \mathrm{CDCl}_{3}\right)$.

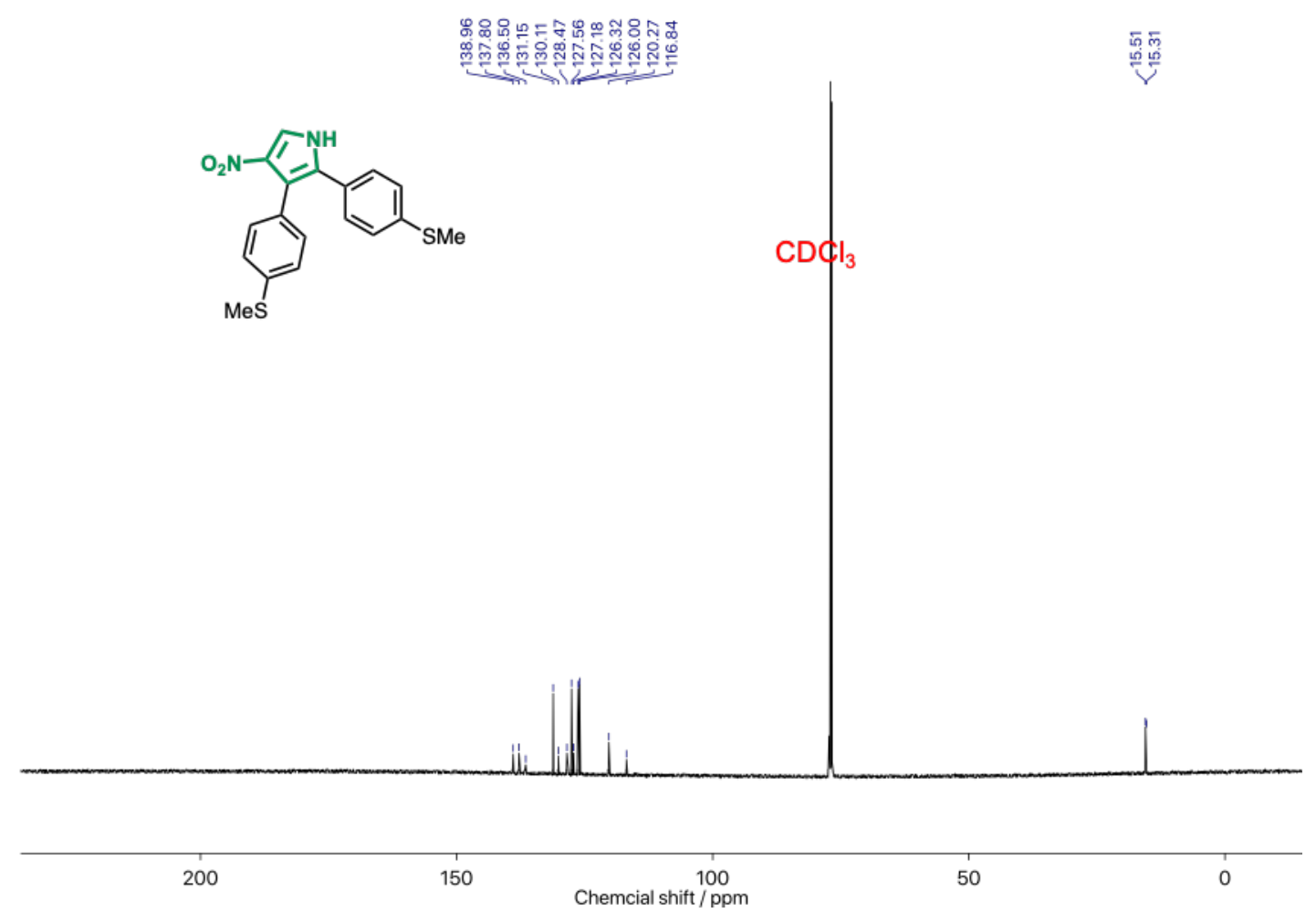

Figure S56. ${ }^{13} \mathrm{C}$ NMR spectrum of PRP $\left(125 \mathrm{MHz}, \mathrm{CDCl}_{3}\right)$. 


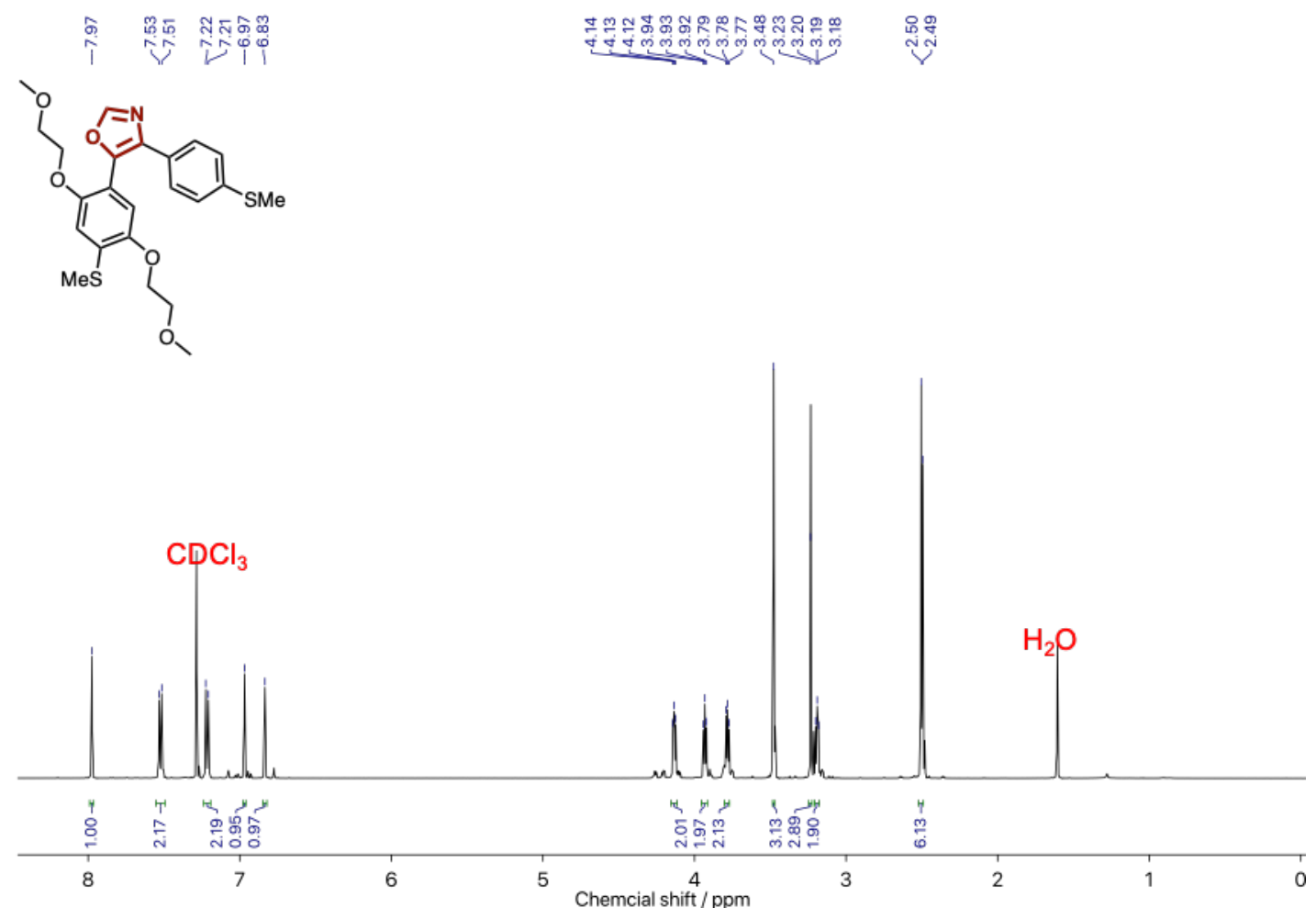

Figure S57. ${ }^{1} \mathrm{H} \mathrm{NMR}$ spectrum of GOP $\left(500 \mathrm{MHz}, \mathrm{CDCl}_{3}\right)$.
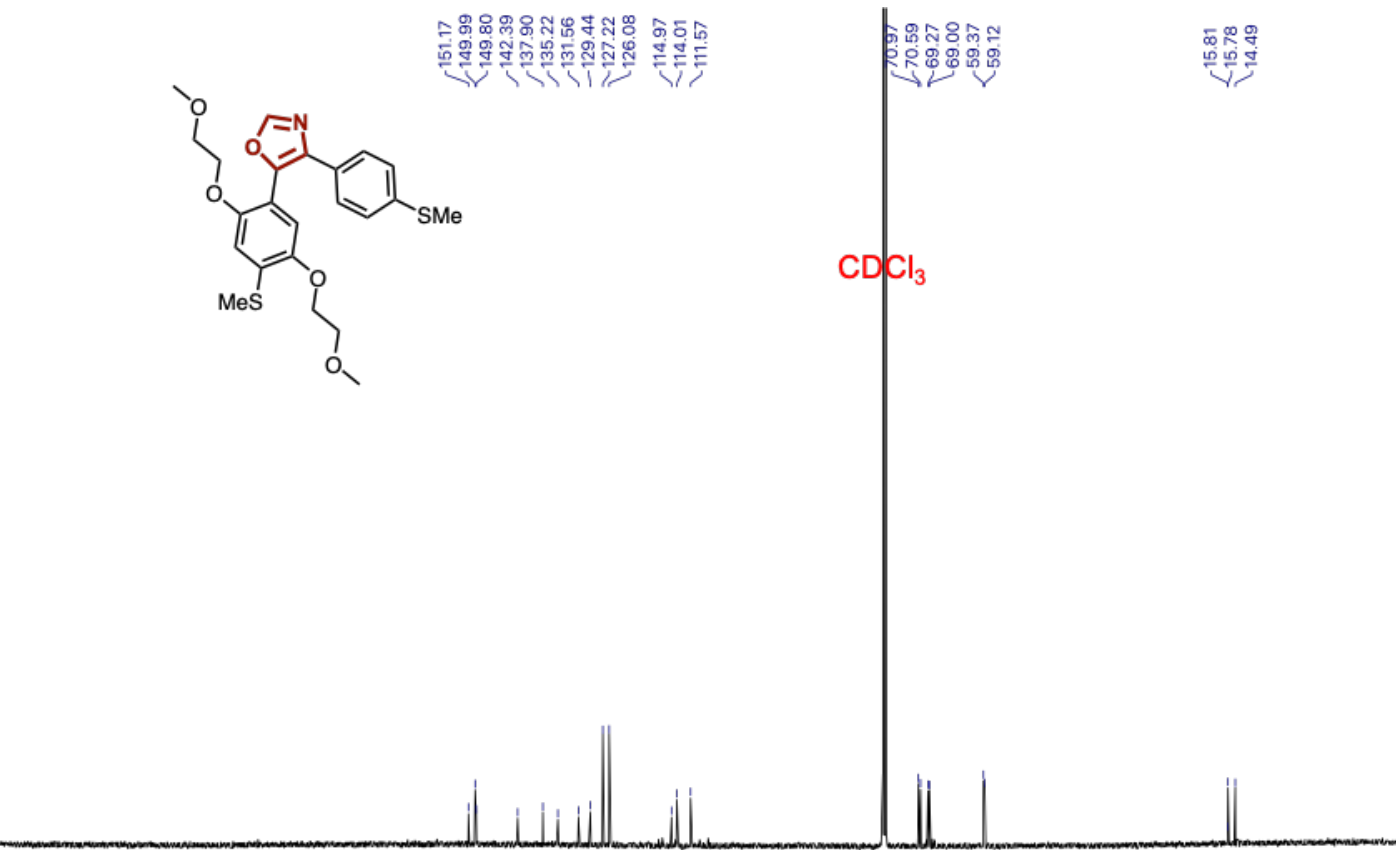

200

Chemcial shift / ppm

50

0

Figure S58. ${ }^{13} \mathrm{C}$ NMR spectrum of GOP $\left(125 \mathrm{MHz}, \mathrm{CDCl}_{3}\right)$. 


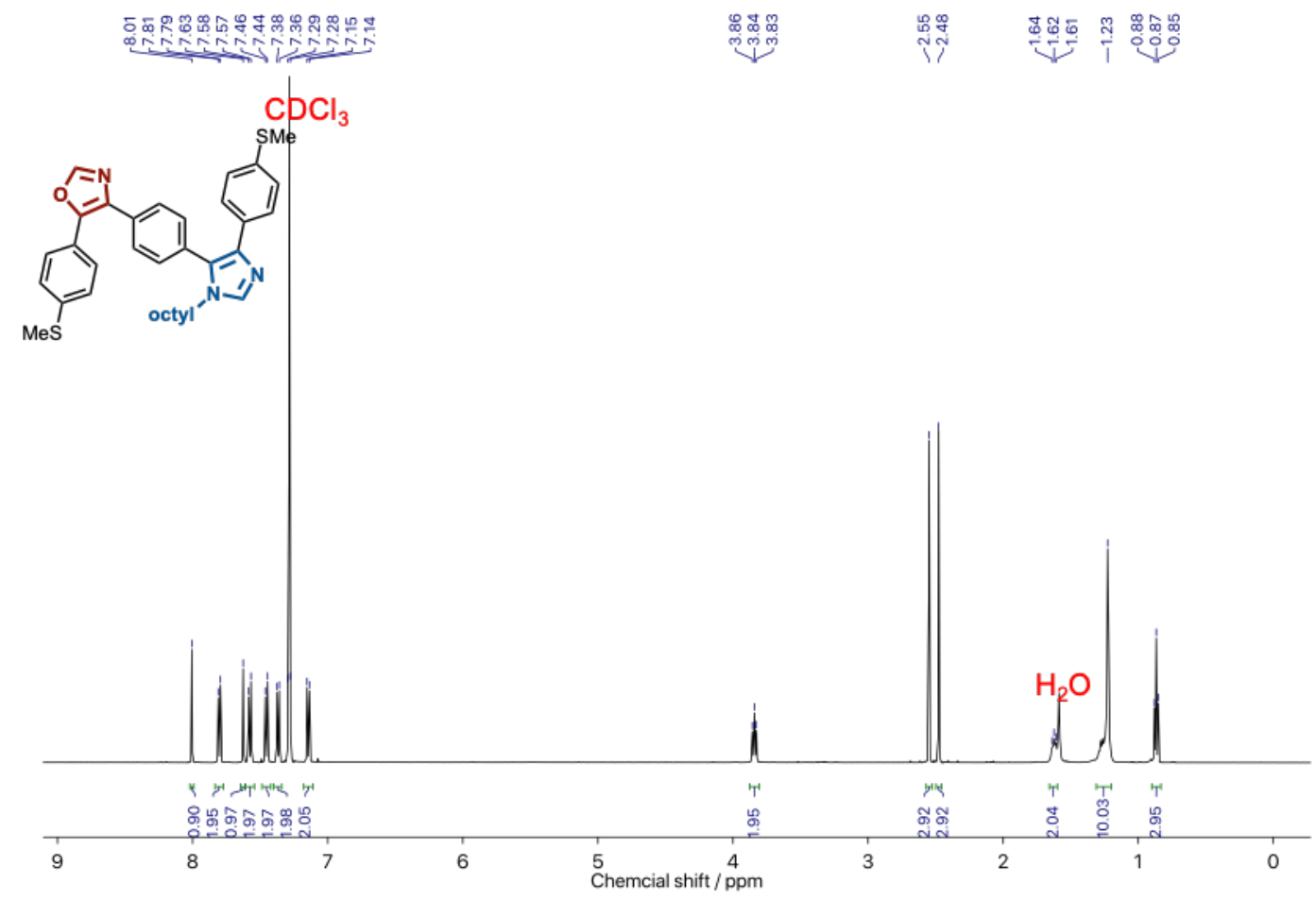

Figure S59. ${ }^{1} \mathrm{H}$ NMR spectrum of POPIP $\left(500 \mathrm{MHz}, \mathrm{CDCl}_{3}\right)$.

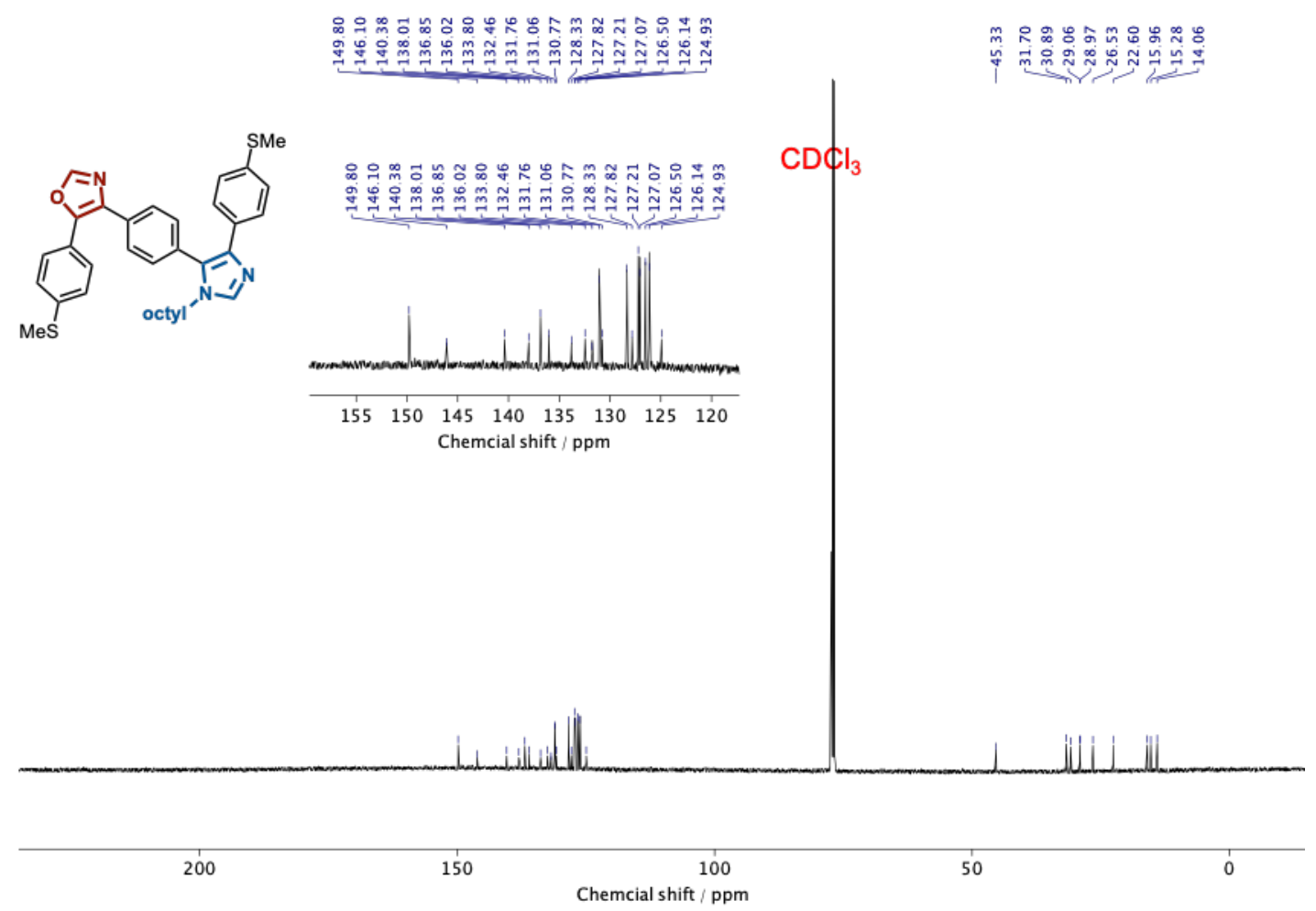

Figure S60. ${ }^{13} \mathrm{C}$ NMR spectrum of POPIP $\left(125 \mathrm{MHz}, \mathrm{CDCl}_{3}\right)$. 


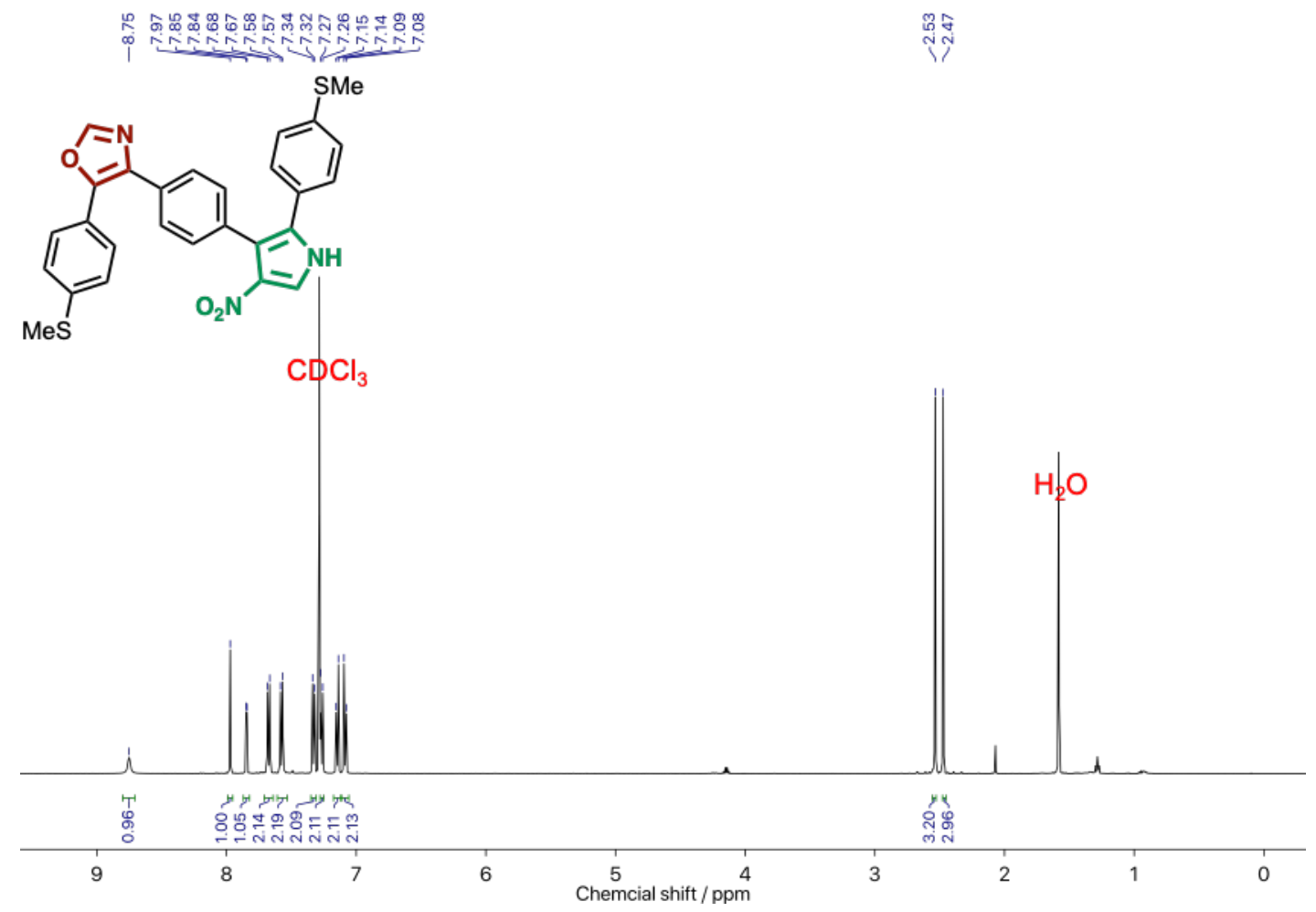

Figure S61. ${ }^{1} \mathrm{H}$ NMR spectrum of POPRP $\left(500 \mathrm{MHz}, \mathrm{CDCl}_{3}\right)$.

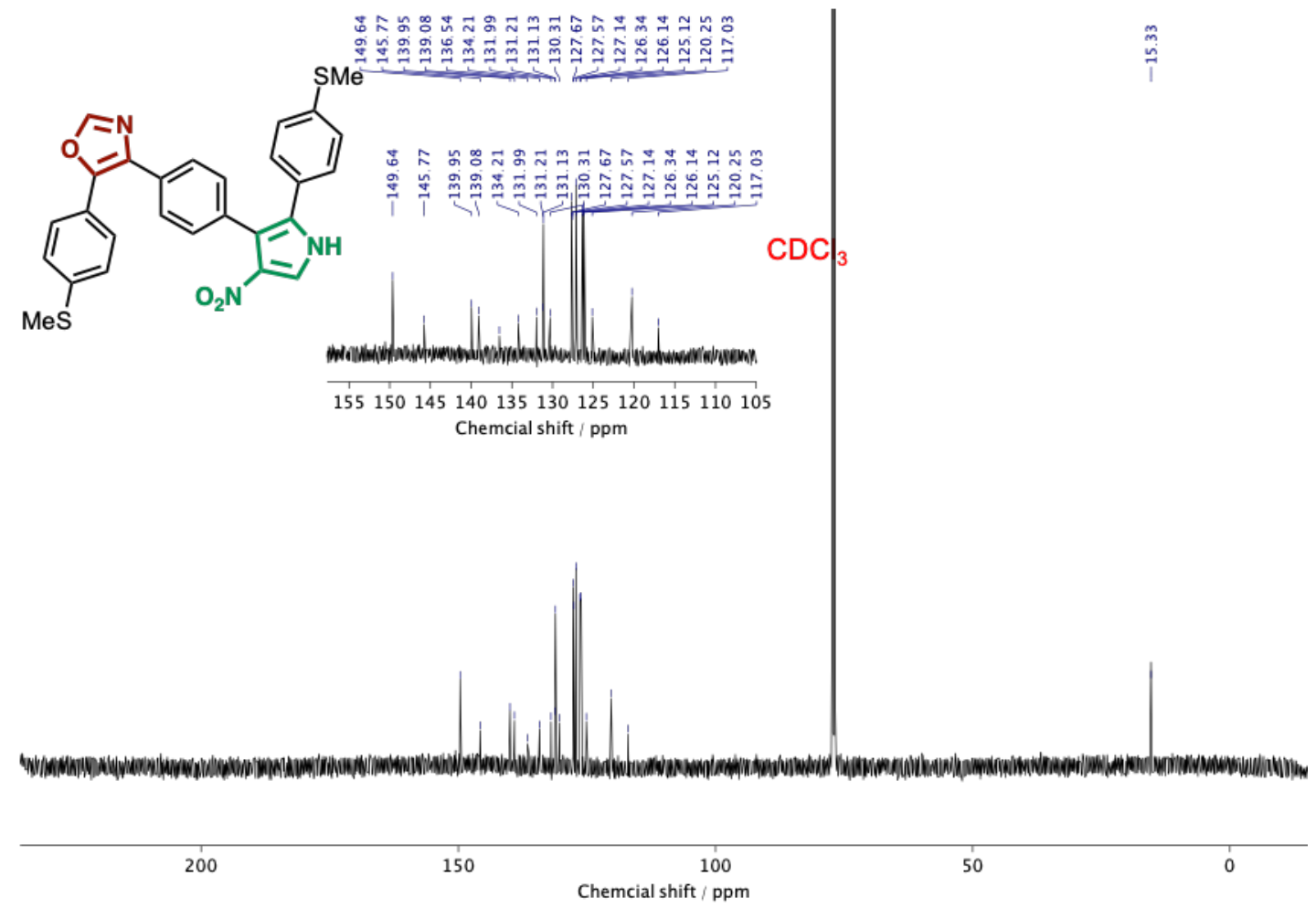

Figure S62. ${ }^{13} \mathrm{C}$ NMR spectrum of POPRP (125 MHz, $\left.\mathrm{CDCl}_{3}\right)$. 


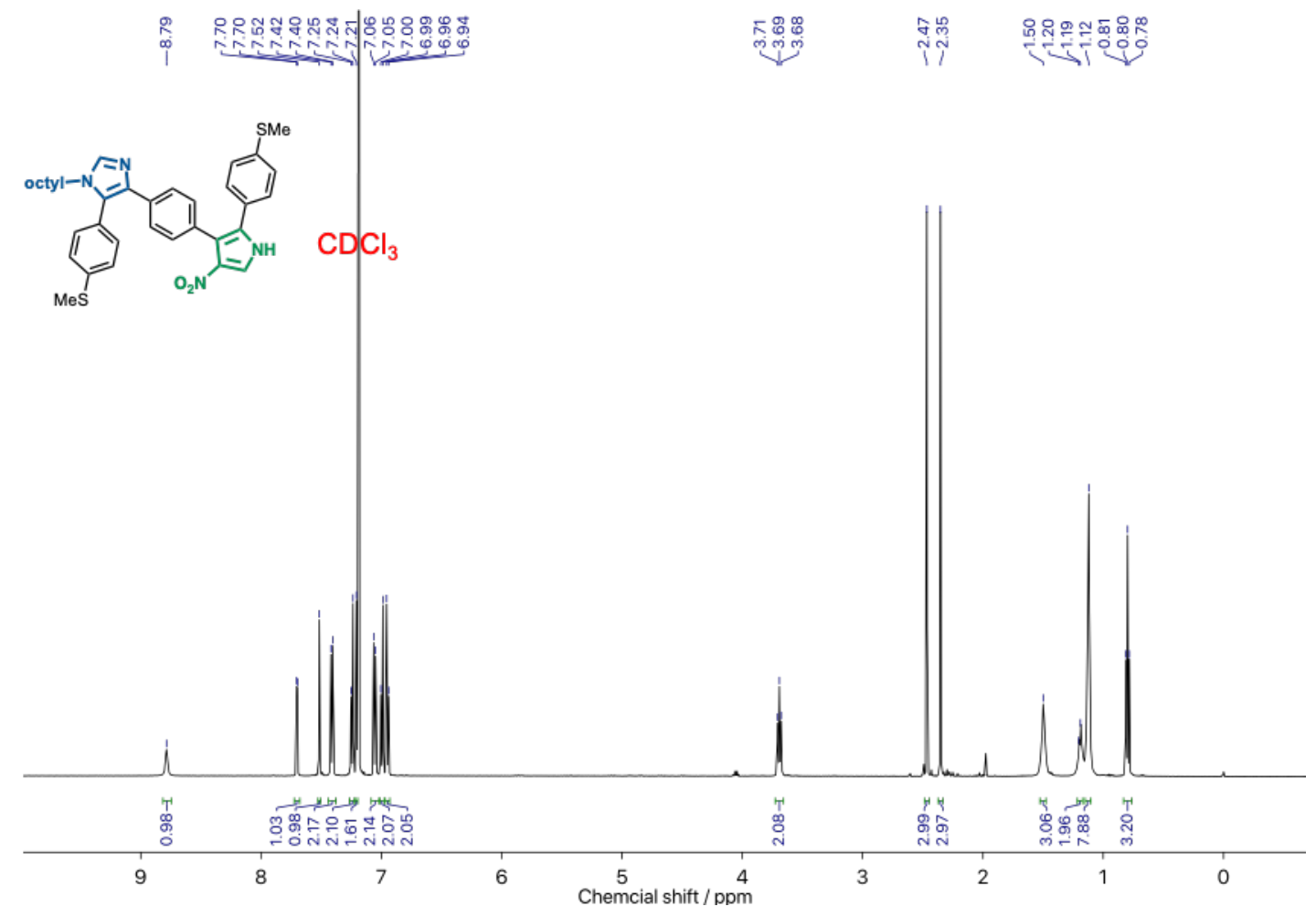

Figure S63. ${ }^{1} \mathrm{H}$ NMR spectrum of PIPRP $\left(500 \mathrm{MHz}, \mathrm{CDCl}_{3}\right)$.

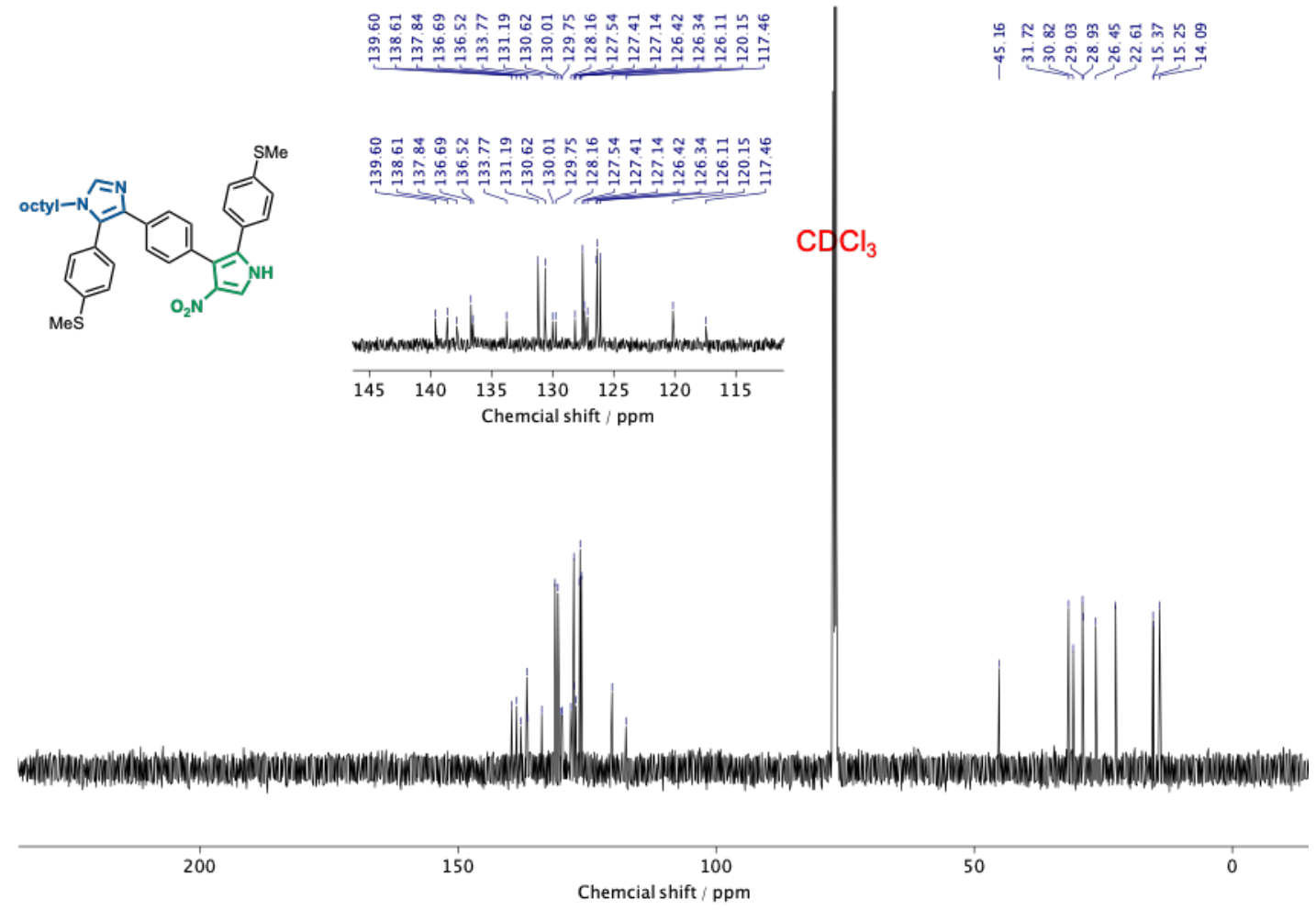

Figure S64. ${ }^{13} \mathrm{C}$ NMR spectrum of PIPRP $\left(125 \mathrm{MHz}, \mathrm{CDCl}_{3}\right)$. 


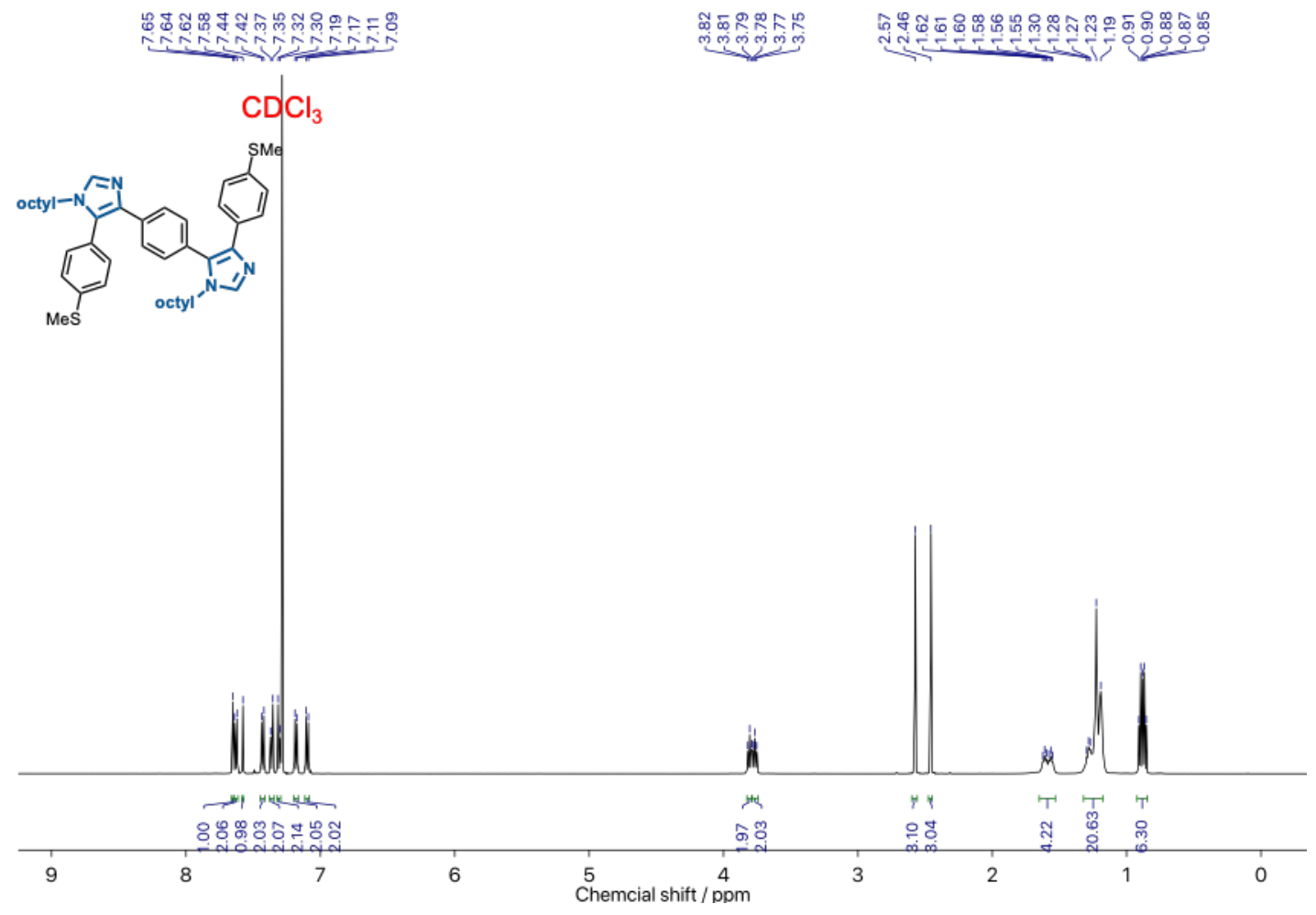

Figure S65. ${ }^{1} \mathrm{H}$ NMR spectrum of PIPIP $\left(500 \mathrm{MHz}, \mathrm{CDCl}_{3}\right)$.

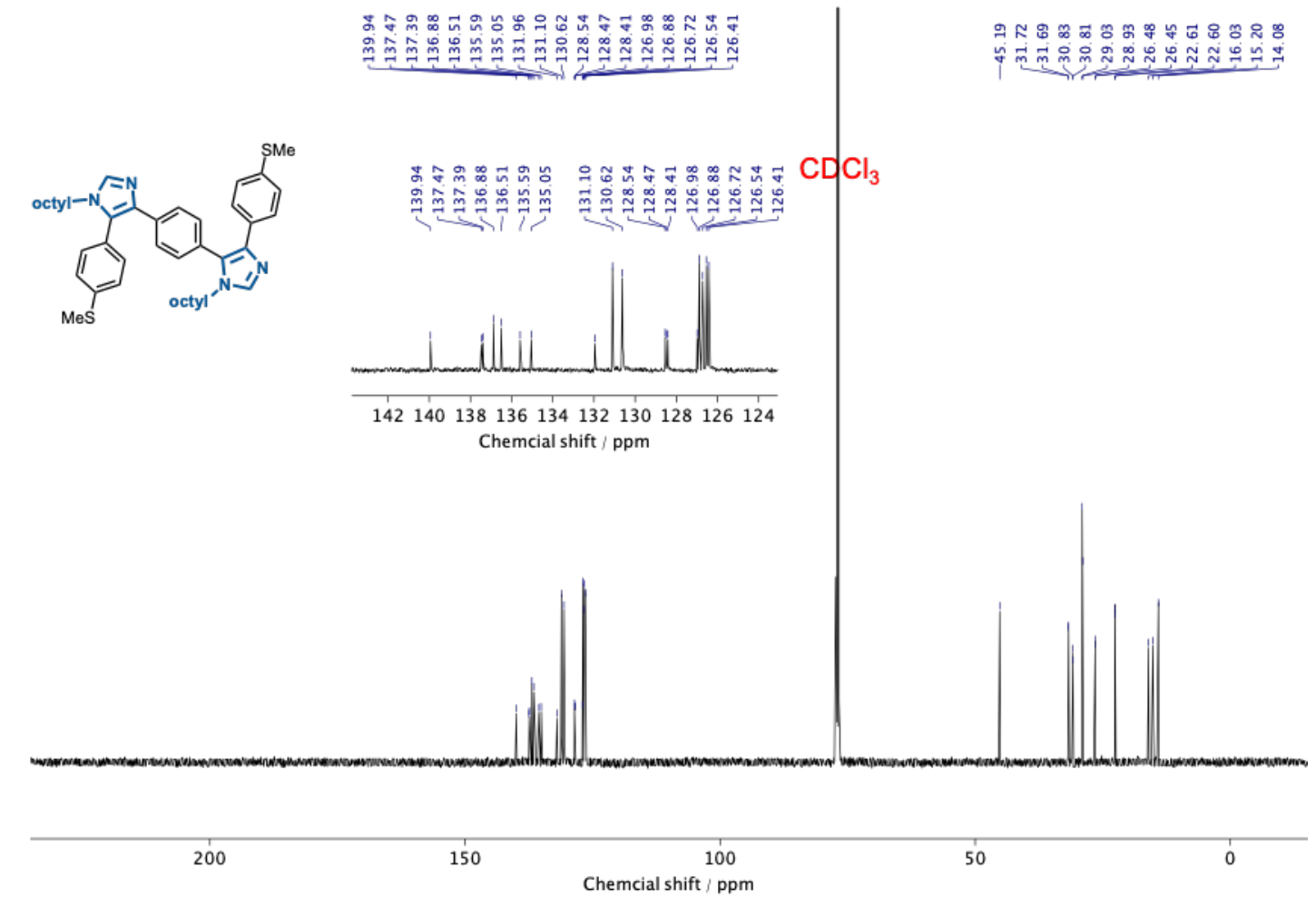

Figure S66. ${ }^{13} \mathrm{C}$ NMR spectrum of PIPIP (125 $\left.\mathrm{MHz}, \mathrm{CDCl}_{3}\right)$. 


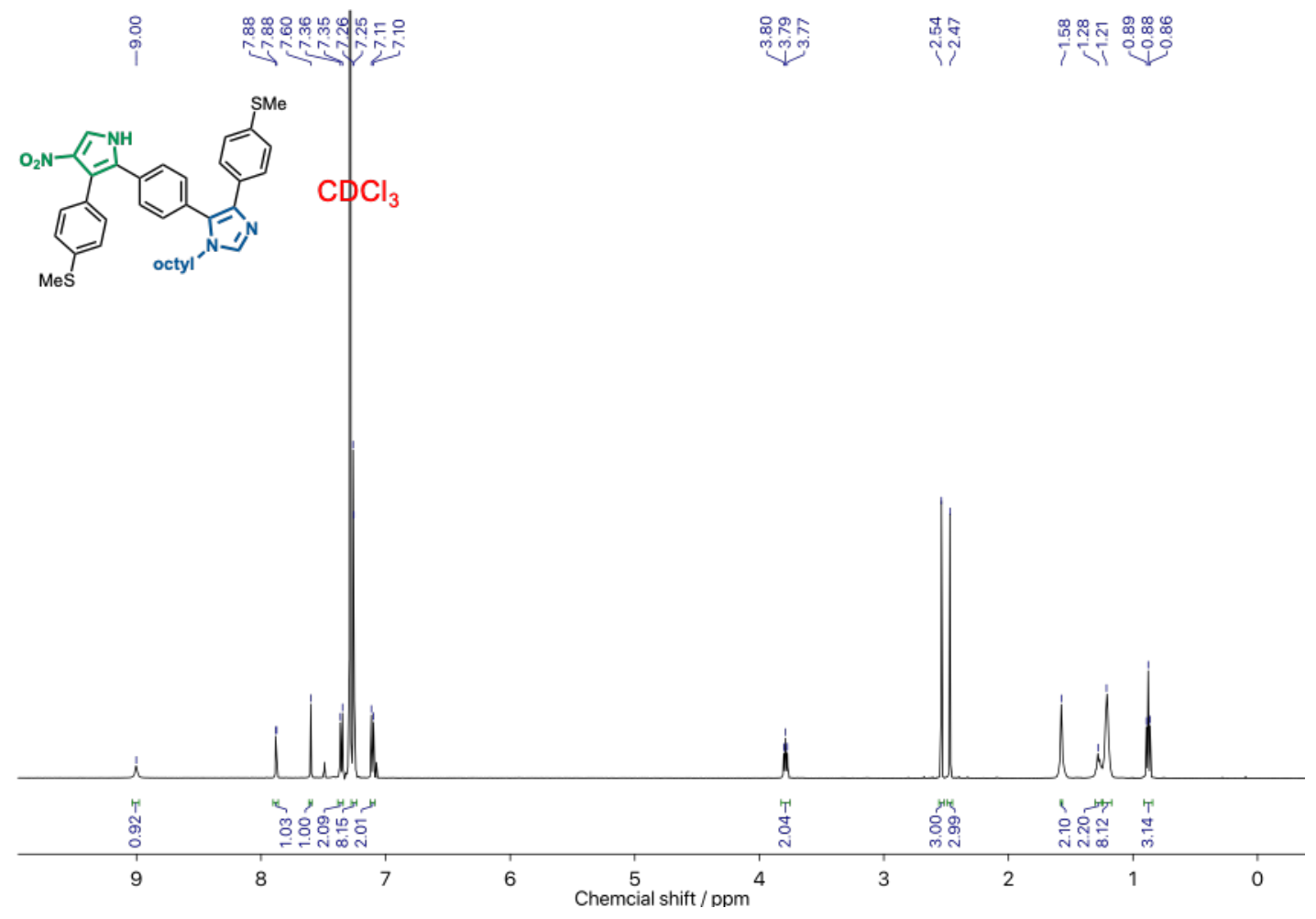

Figure S67. ${ }^{1} \mathrm{H}$ NMR spectrum of PRPIP $\left(500 \mathrm{MHz}, \mathrm{CDCl}_{3}\right)$.

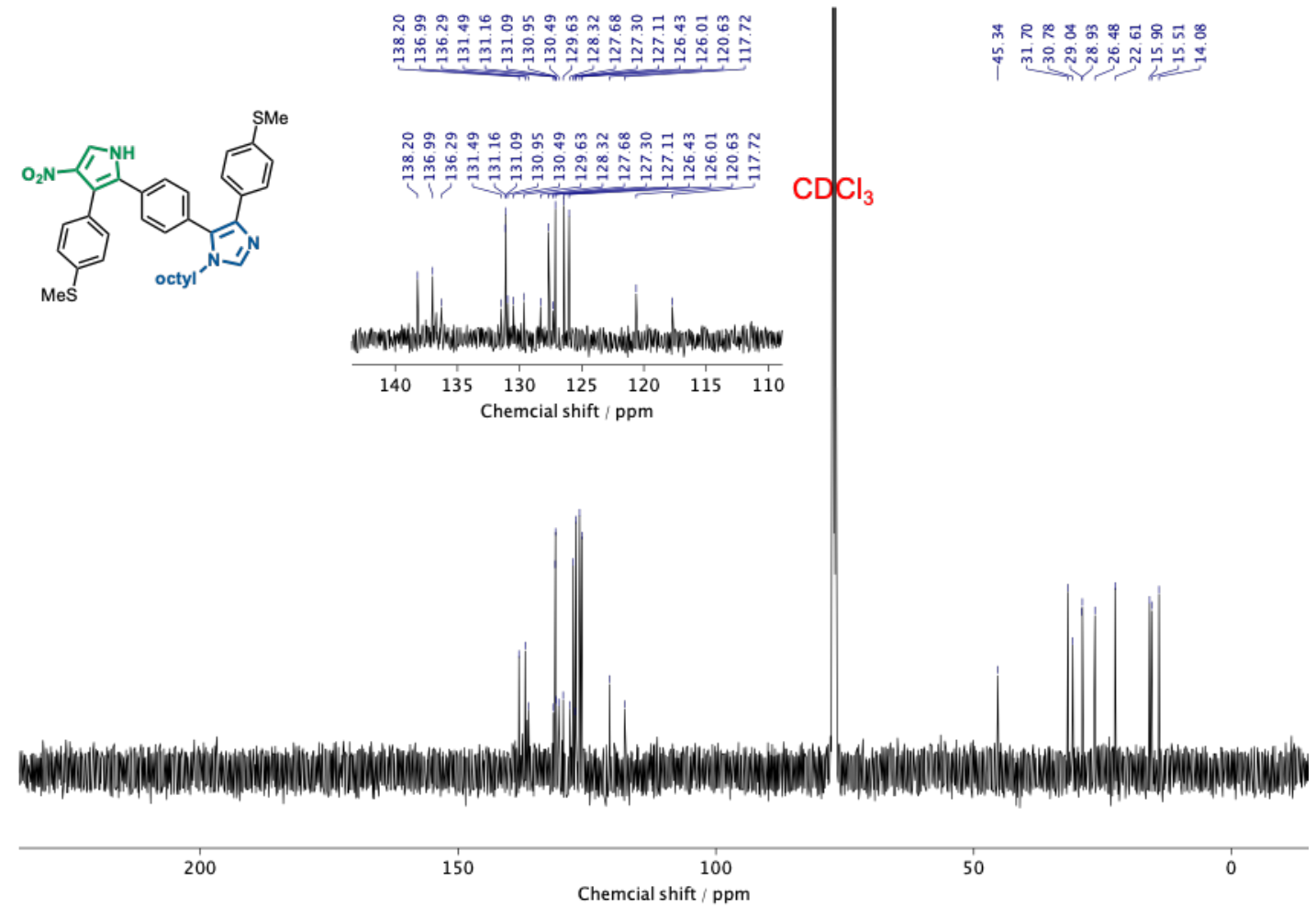

Figure S68. ${ }^{13} \mathrm{C}$ NMR spectrum of PRPIP $\left(125 \mathrm{MHz}, \mathrm{CDCl}_{3}\right)$. 


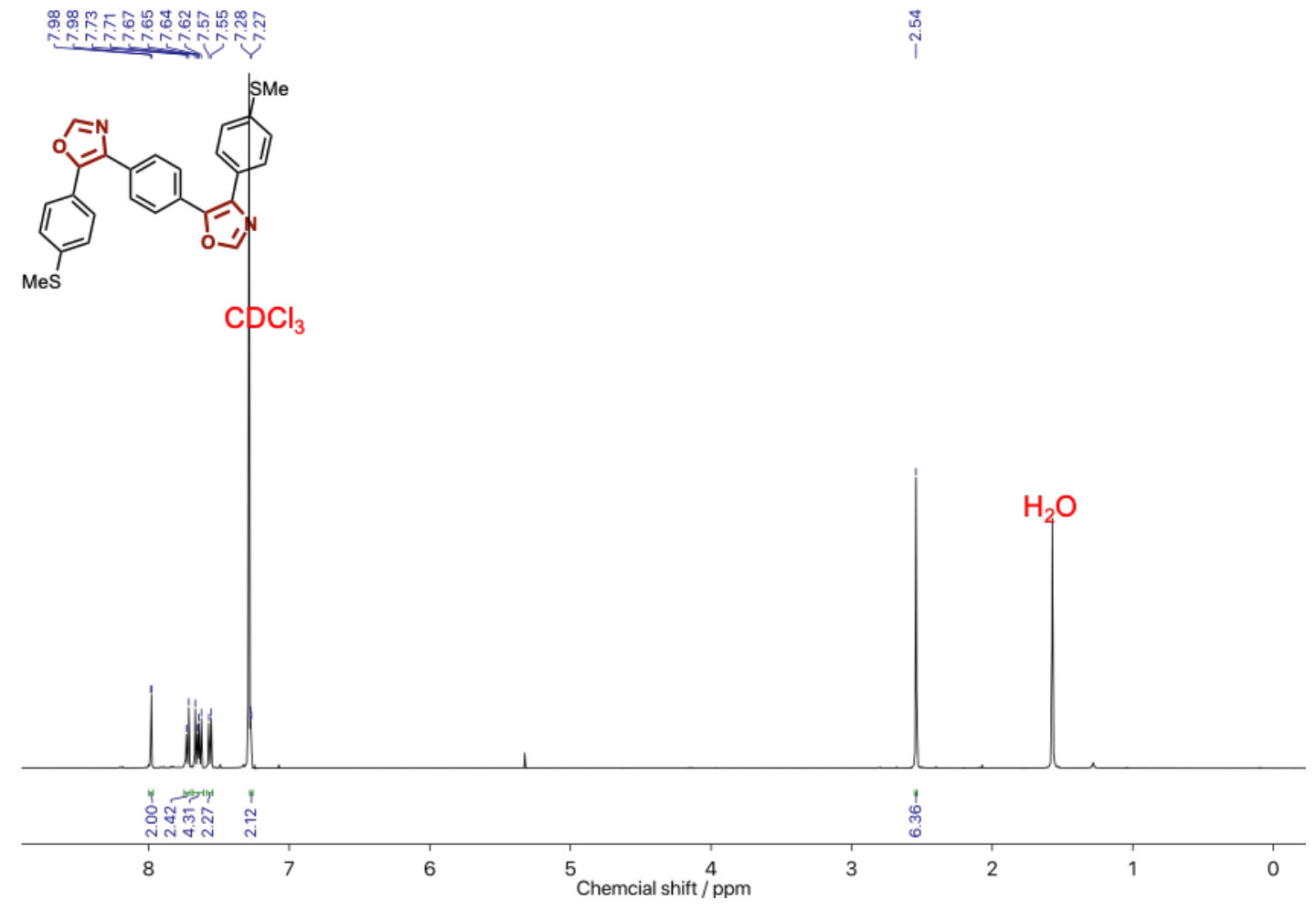

Figure S69. ${ }^{1} \mathrm{H}$ NMR spectrum of POPOP $\left(500 \mathrm{MHz}, \mathrm{CDCl}_{3}\right)$.

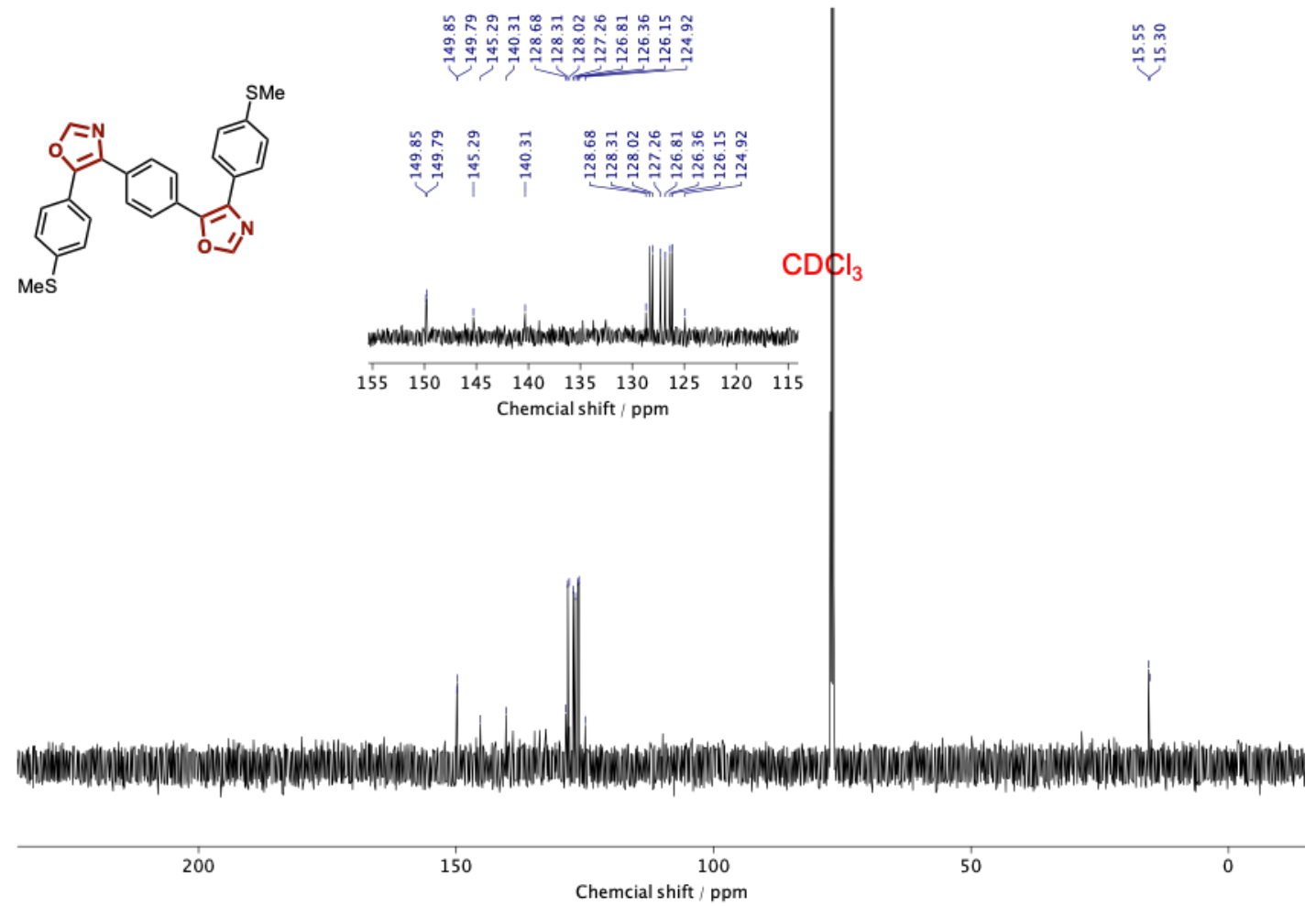

Figure S70. ${ }^{13} \mathrm{C}$ NMR spectrum of POPOP (125 MHz, $\left.\mathrm{CDCl}_{3}\right)$. 


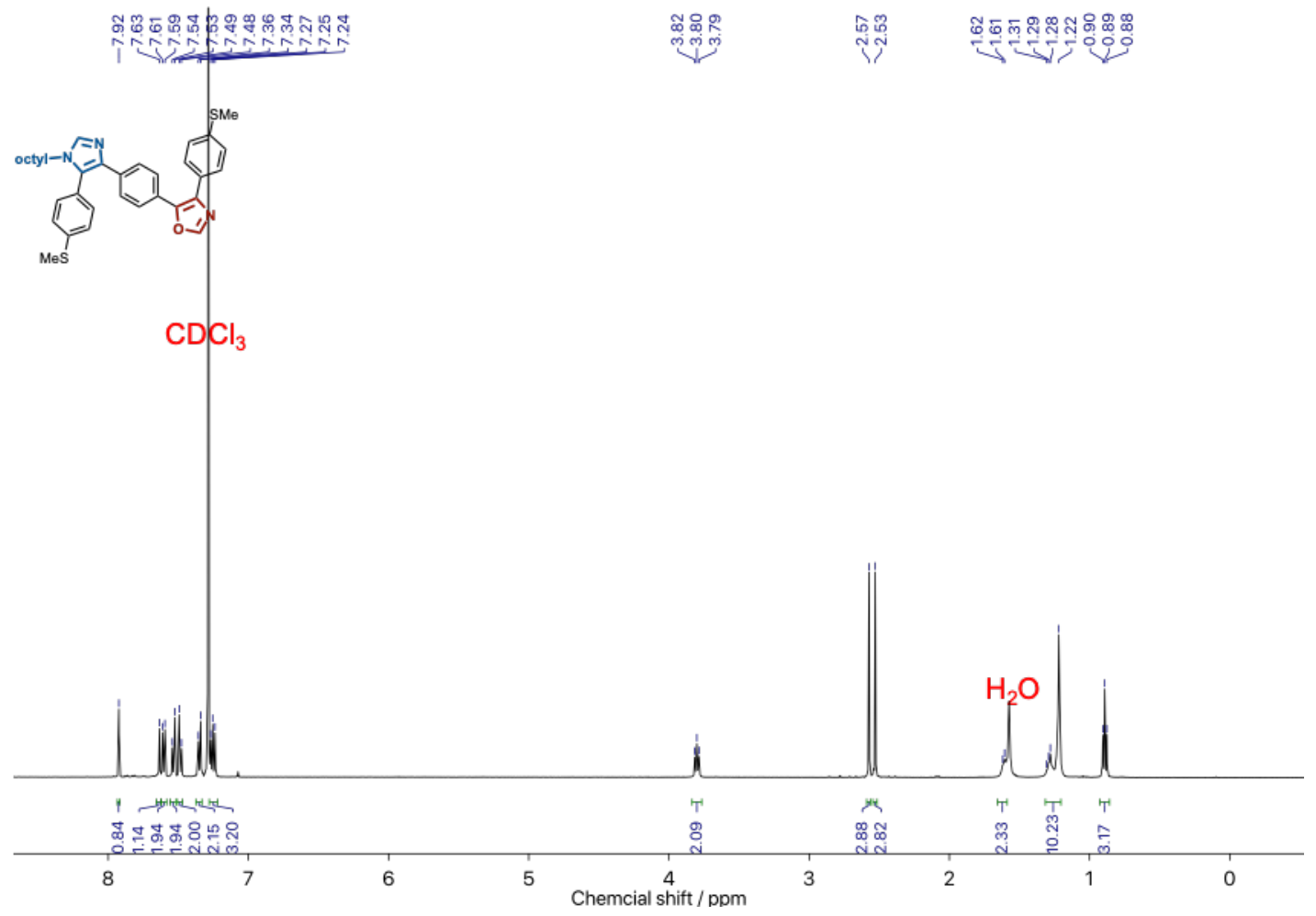

Figure S71. ${ }^{1} \mathrm{H}$ NMR spectrum of PIPOP $\left(500 \mathrm{MHz}, \mathrm{CDCl}_{3}\right)$.

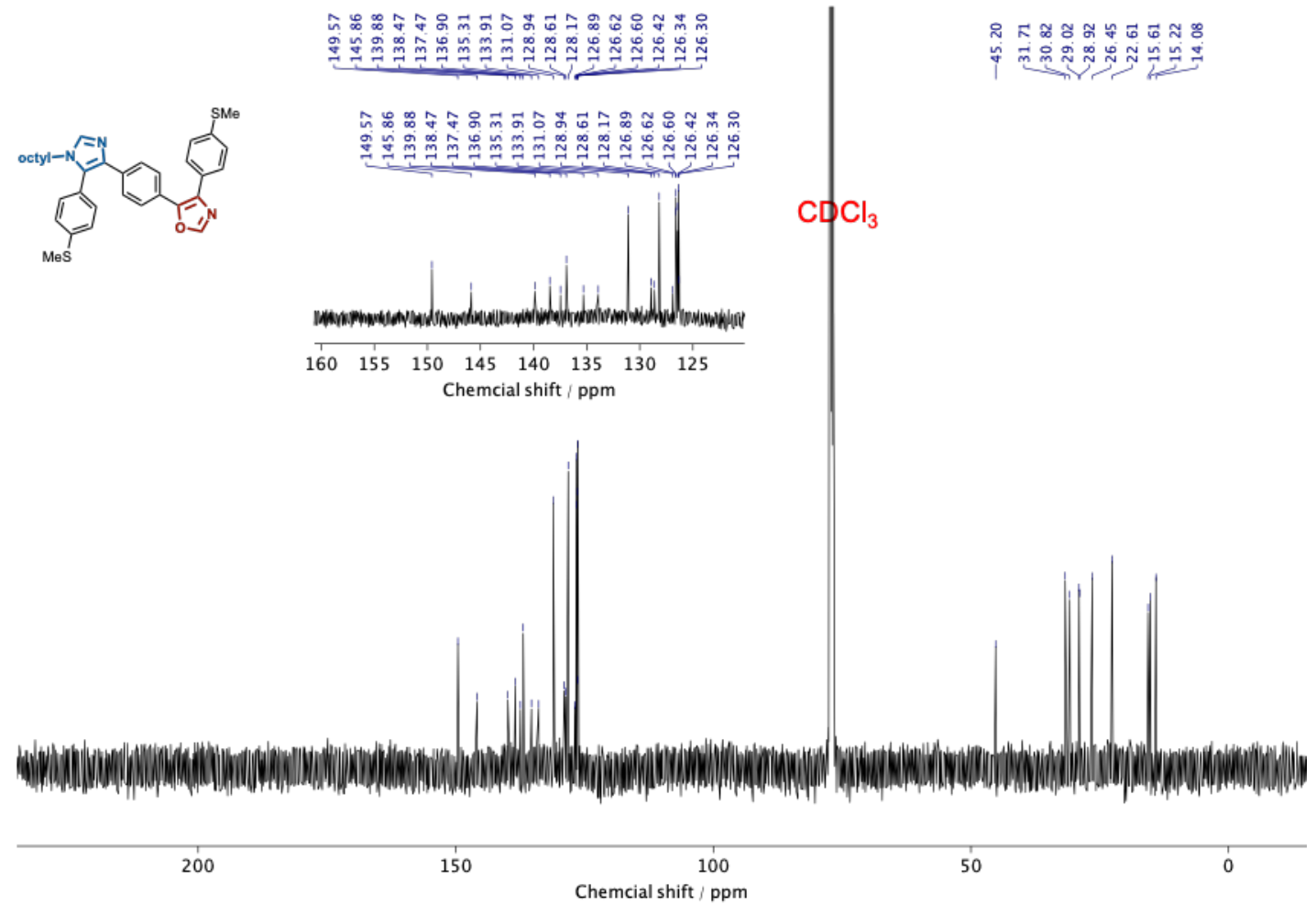

Figure S72. ${ }^{13} \mathrm{C}$ NMR spectrum of PIPOP $\left(125 \mathrm{MHz}, \mathrm{CDCl}_{3}\right)$. 


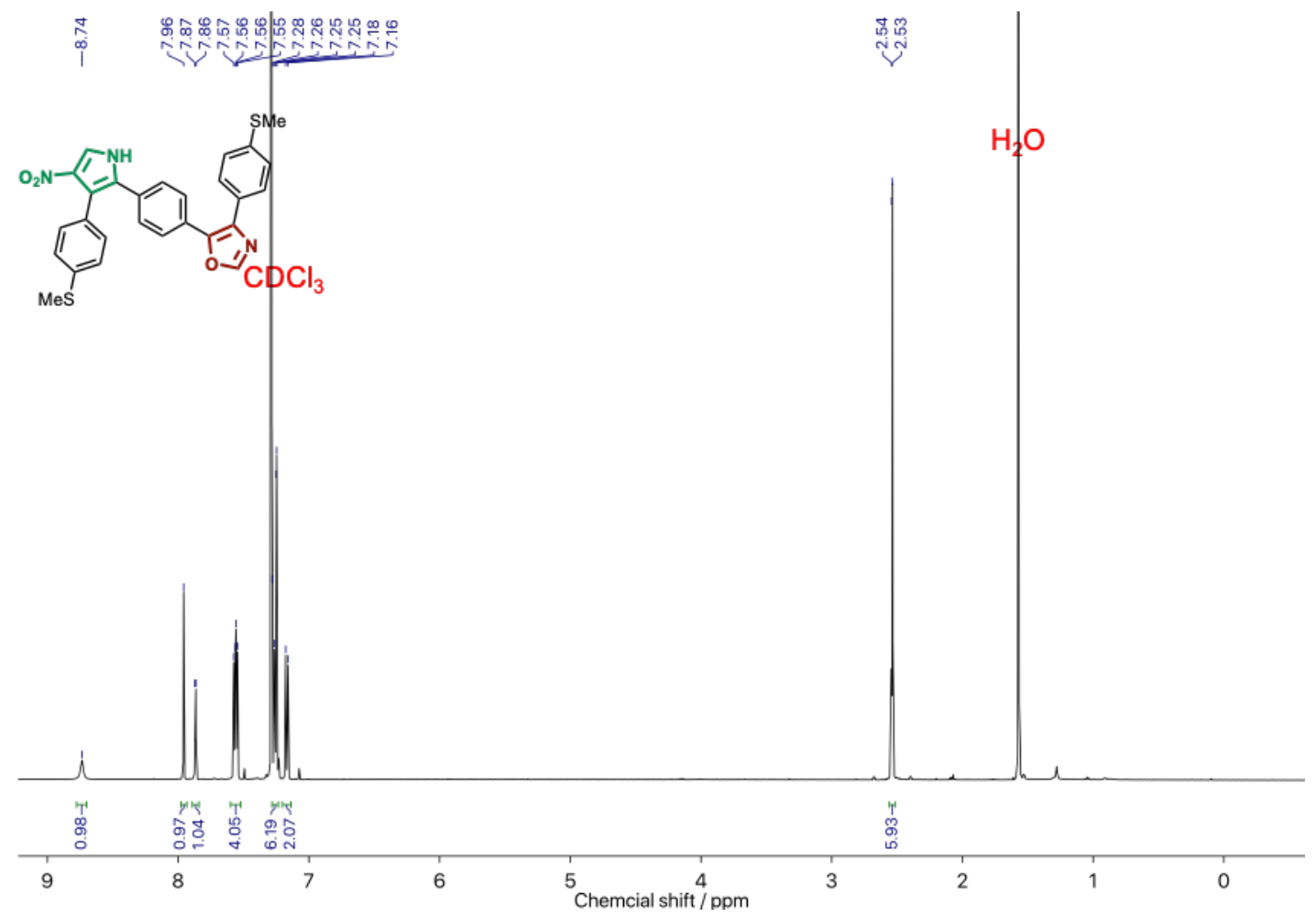

Figure S73. ${ }^{1} \mathrm{H}$ NMR spectrum of PRPOP $\left(500 \mathrm{MHz}, \mathrm{CDCl}_{3}\right)$.

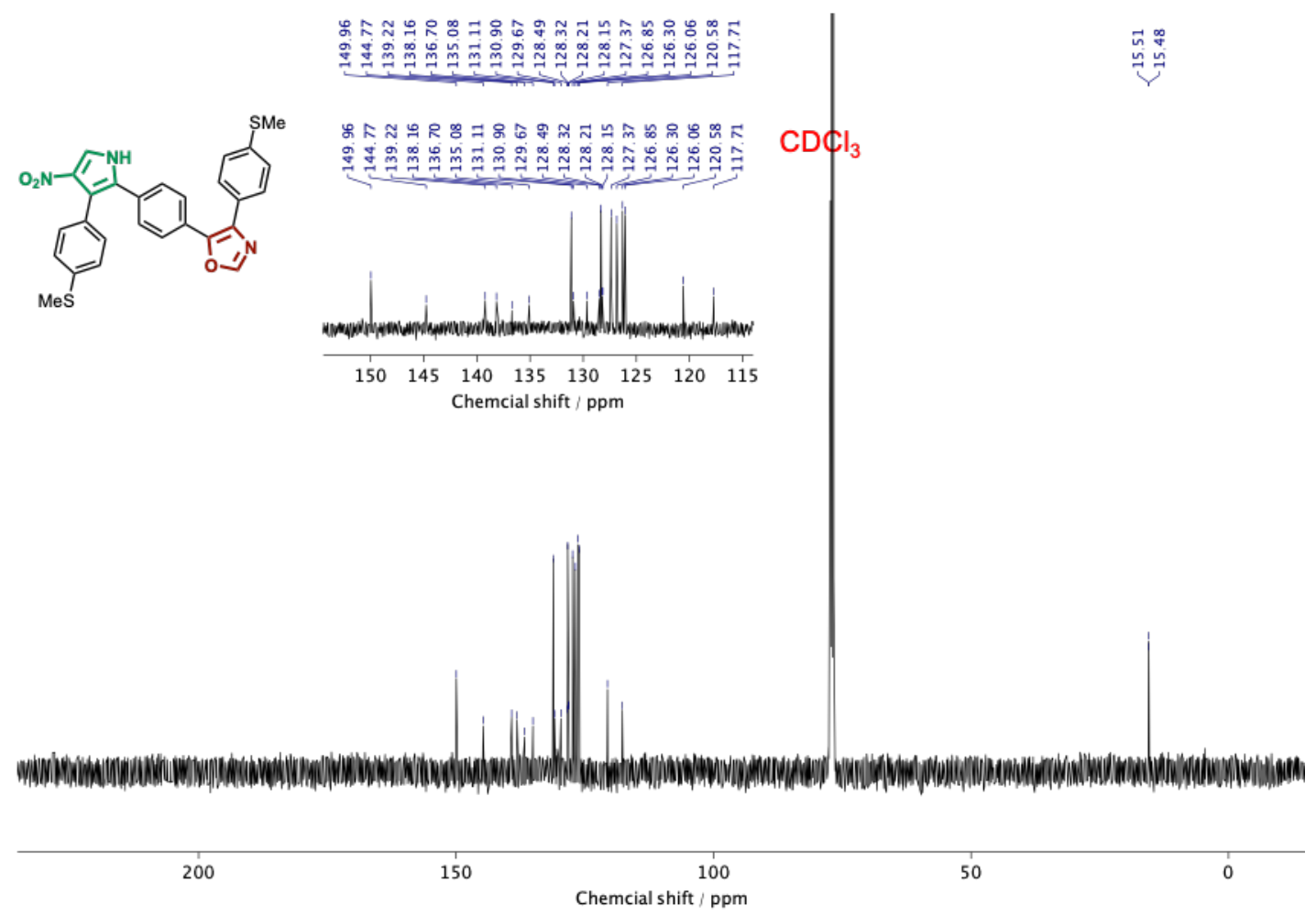

Figure S74. ${ }^{13} \mathrm{C}$ NMR spectrum of PRPOP $\left(125 \mathrm{MHz}, \mathrm{CDCl}_{3}\right)$. 


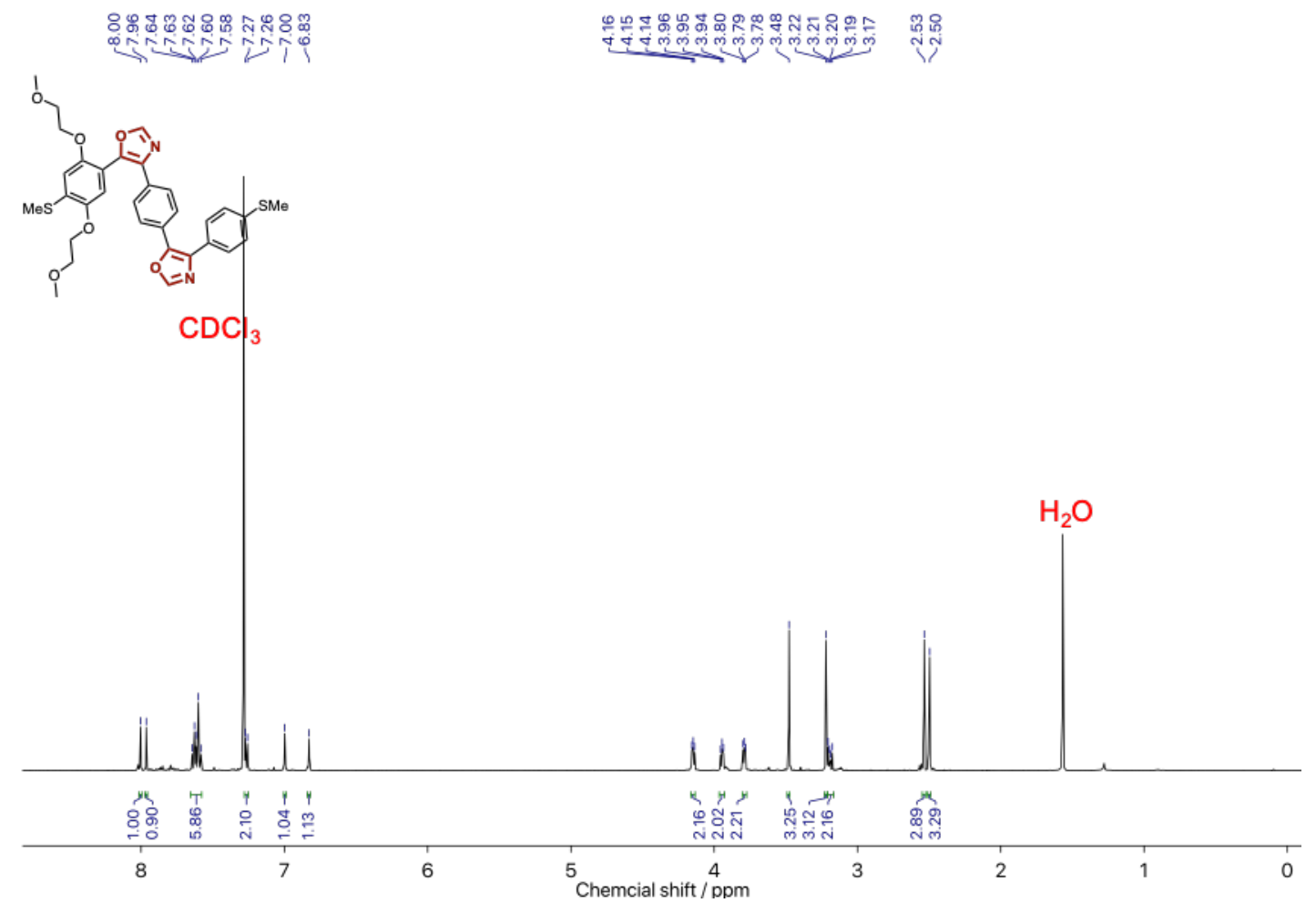

Figure S75. ${ }^{1} \mathrm{H}$ NMR spectrum of GOPOP $\left(500 \mathrm{MHz}, \mathrm{CDCl}_{3}\right)$.

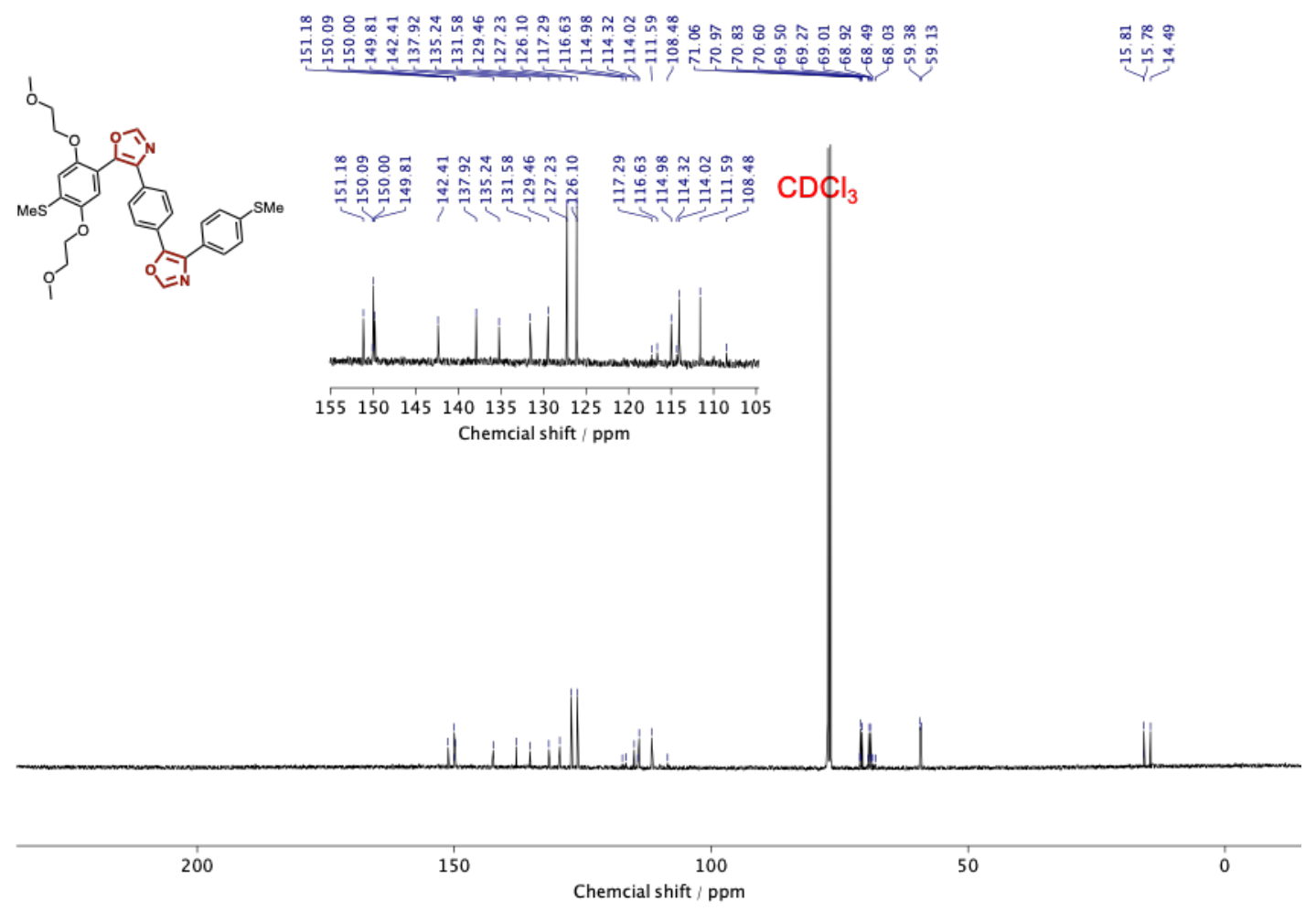

Figure S76. ${ }^{13} \mathrm{C}$ NMR spectrum of GOPOP $\left(125 \mathrm{MHz}, \mathrm{CDCl}_{3}\right)$. 


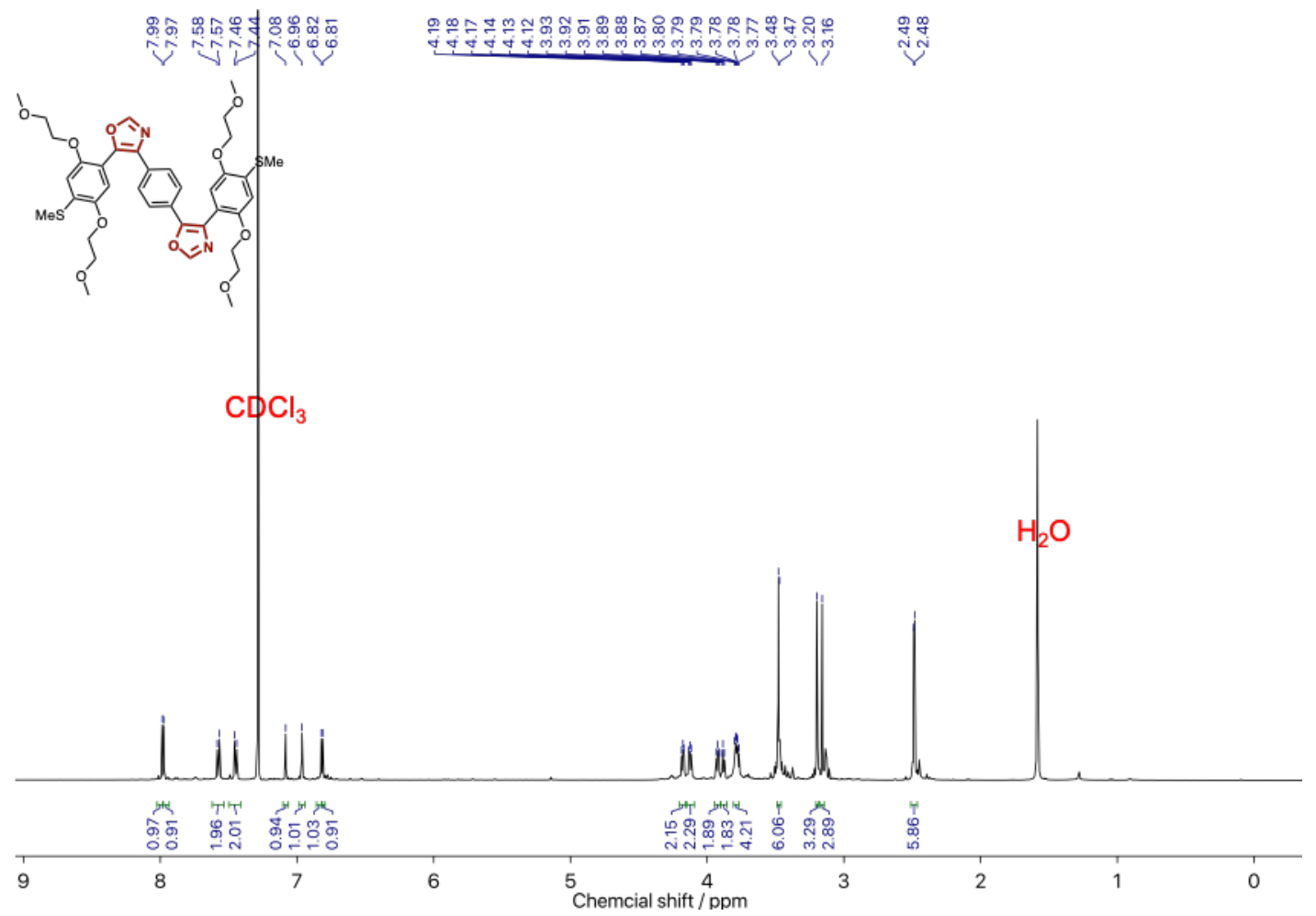

Figure S77. ${ }^{1} \mathrm{H}$ NMR spectrum of GOPO'G (500 MHz, $\left.\mathrm{CDCl}_{3}\right)$.

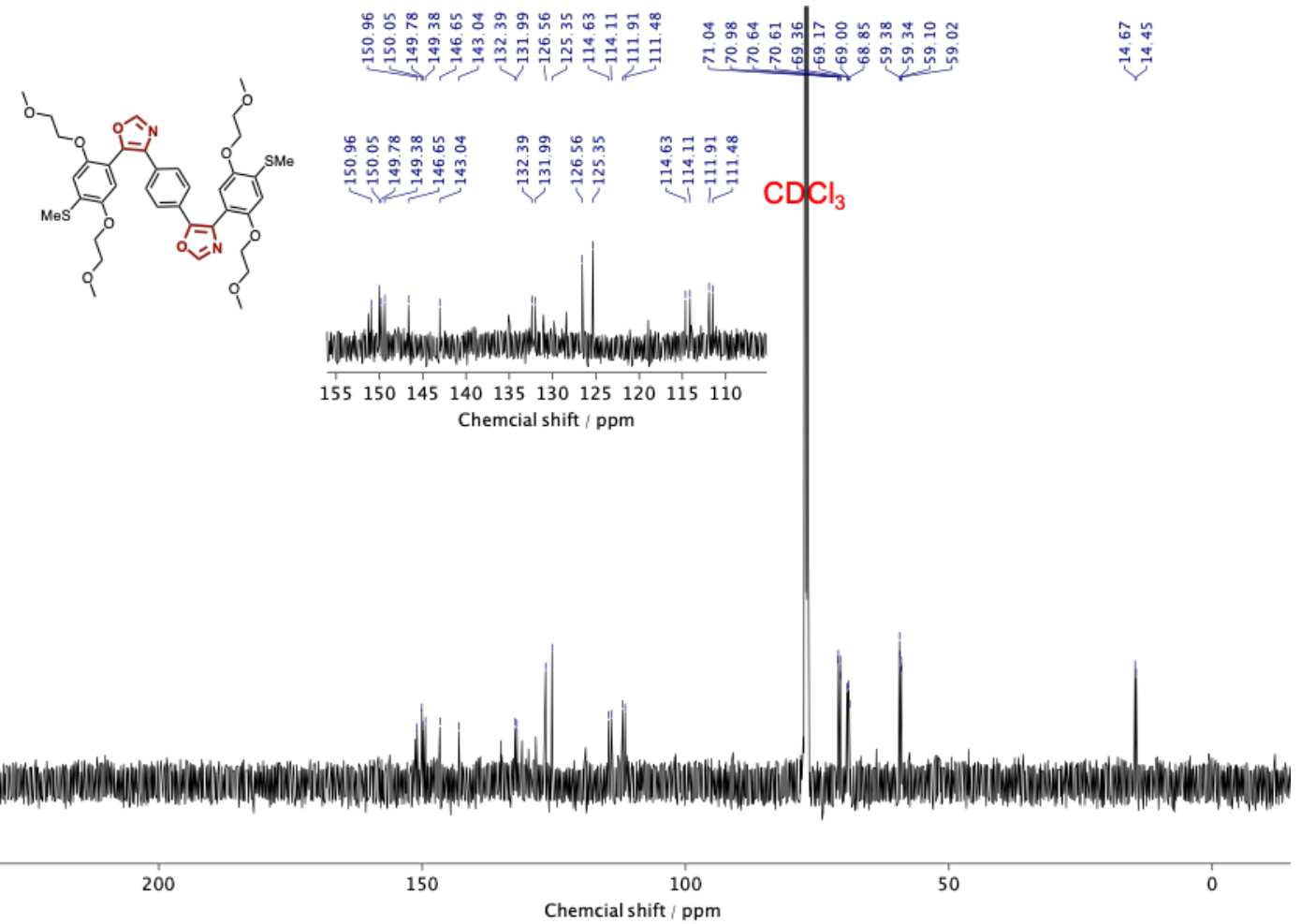

Figure S78. ${ }^{13} \mathrm{C}$ NMR spectrum of GOPOG (125 MHz, $\left.\mathrm{CDCl}_{3}\right)$. 


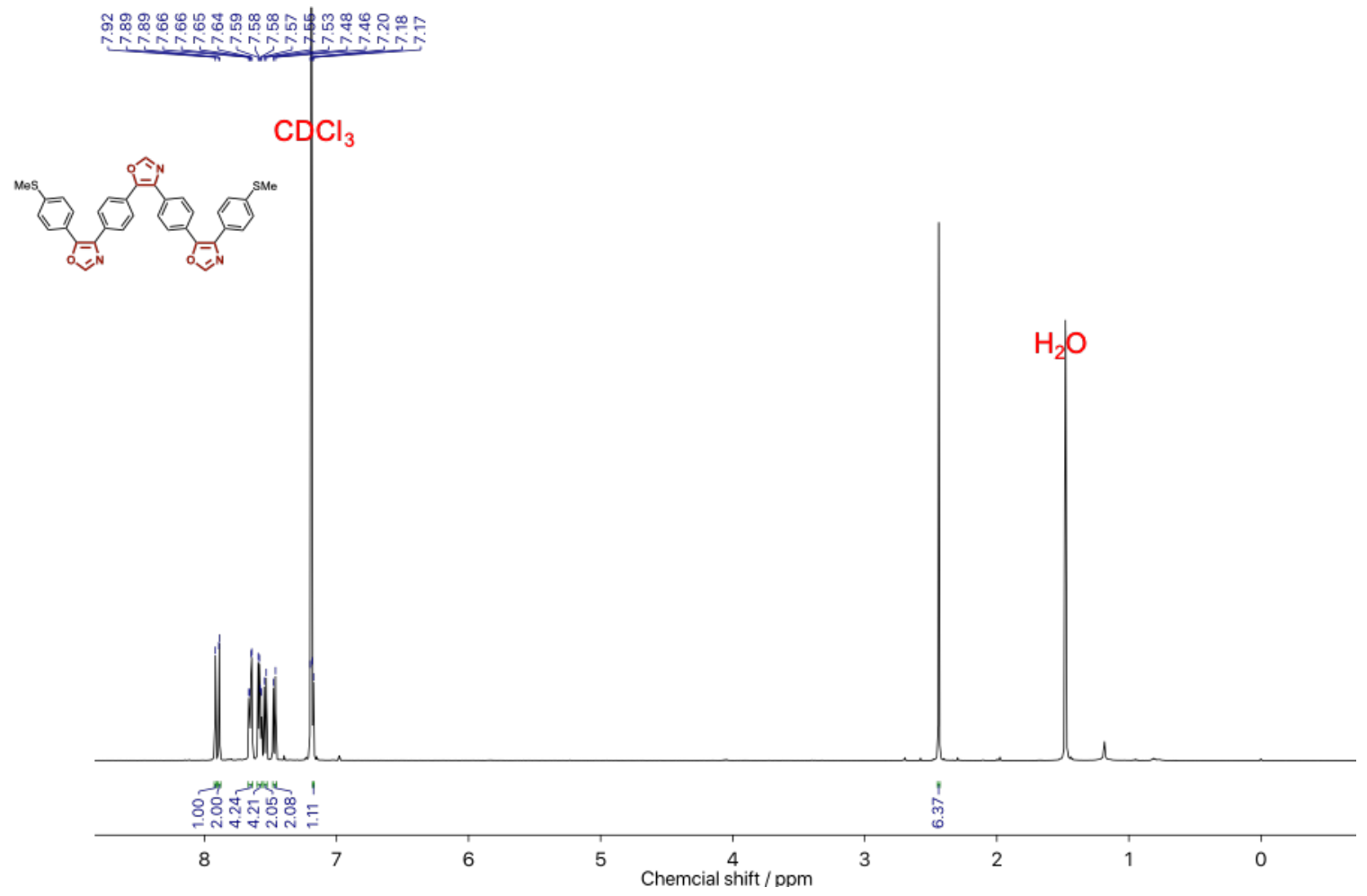

Figure S79. ${ }^{1} \mathrm{H}$ NMR spectrum of POPOPOP $\left(500 \mathrm{MHz}, \mathrm{CDCl}_{3}\right)$.

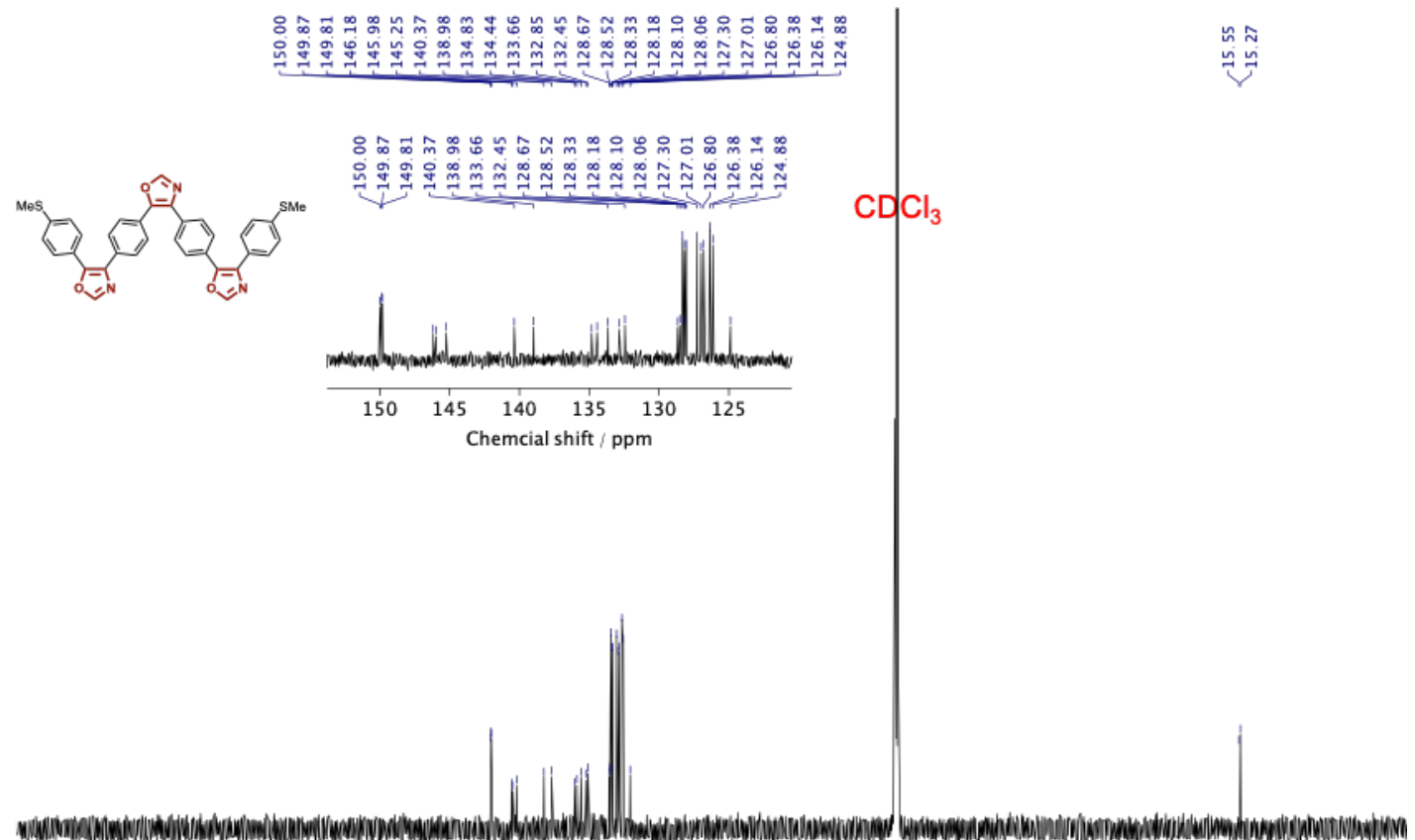

200

150

Chemcial shift $/ \mathrm{ppm}$

Figure S80. ${ }^{13} \mathrm{C}$ NMR spectrum of POPOPOP (125 MHz, $\left.\mathrm{CDCl}_{3}\right)$. 


\section{Single Crystal X-ray Crystallographic Analysis of POPRP and PRPOP}

\subsection{Single Crystal Data of POPRP (crystal obtained from a solution mixture of $\mathrm{CHCl}_{3} / \mathrm{n}$ - Hexane)}

Table S1. Crystal data and structure refinement for POPRP.

Identification code

Empirical formula

Formula weight

Temperature

Wavelength

Crystal system

Space group

Unit cell dimensions

Volume

Z

Density (calculated)

Absorption coefficient

$\mathrm{F}(000)$

Crystal size

Theta range for data collection

Index ranges

Reflections collected

Independent reflections

Completeness to theta $=25.242^{\circ}$

Absorption correction

Max. and min. transmission

Refinement method

Data / restraints / parameters

Goodness-of-fit on $\mathrm{F}^{2}$

Final $\mathrm{R}$ indices [I $>2 \operatorname{sigma}(\mathrm{I})]$

$\mathrm{R}$ indices (all data)

Extinction coefficient

Largest diff. peak and hole $\quad 0.325$ and -0.315 e. $\AA^{-3}$

499.59

100(2) K

$0.71073 \AA$

Monoclinic

$\mathrm{P} 21 / \mathrm{c}$

4

1040

72191

$100.0 \%$

Integration

1.338

$0.0492(17)$

dd40ssa_final

C27 H21 N3 O3 S2

$\mathrm{a}=9.9882(3) \AA \quad \mathrm{a}=90^{\circ}$.

$\mathrm{b}=16.7181(4) \AA \quad \mathrm{b}=107.5010(10)^{\circ}$.

$\mathrm{c}=14.9023(4) \AA \quad \mathrm{g}=90^{\circ}$.

2373.26(11) $\AA^{3}$

$1.398 \mathrm{Mg} / \mathrm{m}^{3}$

$0.260 \mathrm{~mm}^{-1}$

$0.588 \times 0.290 \times 0.270 \mathrm{~mm}^{3}$

2.138 to $27.152^{\circ}$.

$-12<=\mathrm{h}<=12,-21<=\mathrm{k}<=21,-18<=\mathrm{l}<=19$

$5250[\mathrm{R}(\mathrm{int})=0.0524]$

0.9415 and 0.8723

Full-matrix least-squares on $\mathrm{F}^{2}$

5250 / 1863 / 632

$\mathrm{R} 1=0.0672, \mathrm{wR} 2=0.1540$

$\mathrm{R} 1=0.0702, \mathrm{wR} 2=0.1551$ 
(a)

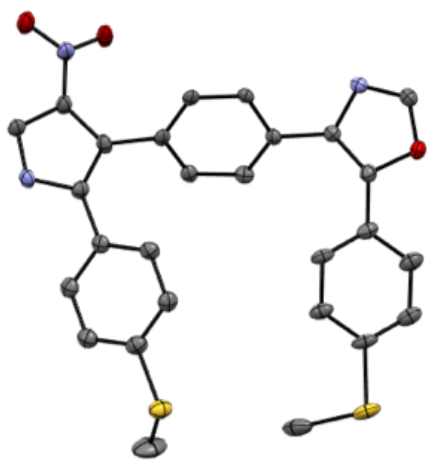

(c)

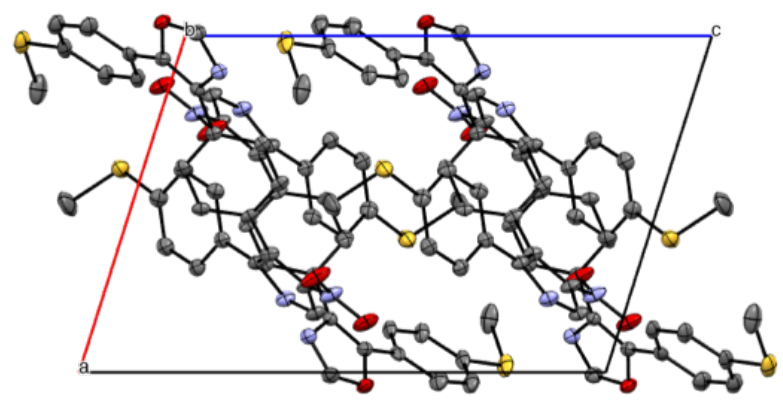

(b)

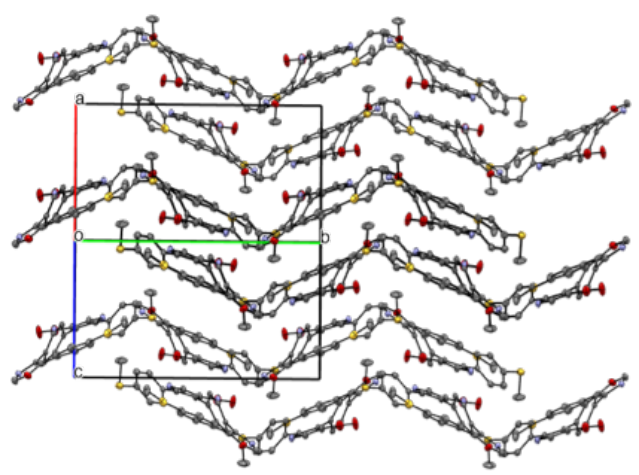

(d)

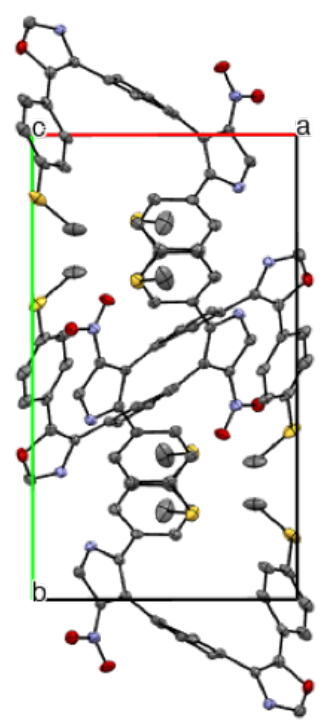

Figure S81. Selected images of POPRP with hydrogens omitted for clarity. (a) Single molecule ellipsoid view of POPRP. (b) Molecule packing along the b-axis (c) Unit cell viewed along the baxis. (d) Unit cell viewed along the c-axis.

\subsection{Single Crystal Data of POPRP (crystal obtained from a solution mixture of EtOAc/n- Hexane)}

Table S2. Crystal data and structure refinement for POPRP.

Identification code

Empirical formula

Formula weight

Temperature

Wavelength

Crystal system

Space group

Unit cell dimensions dd49ss

C27 H21 N3 O3 S2

499.59

100(2) K

$0.71073 \AA$

Monoclinic

$\mathrm{P} 2{ }_{1} / \mathrm{c}$

$$
\begin{array}{ll}
\mathrm{a}=8.8776(2) \AA & \mathrm{a}=90^{\circ} . \\
\mathrm{b}=19.7436(4) \AA & \mathrm{b}=101.6930(6)^{\circ} . \\
\mathrm{c}=13.4135(3) \AA & \mathrm{g}=90^{\circ} .
\end{array}
$$


Volume

$\mathrm{Z}$

Density (calculated)

Absorption coefficient

$\mathrm{F}(000)$

Crystal size

Theta range for data collection

Index ranges

Reflections collected

Independent reflections

Completeness to theta $=25.242^{\circ}$

Absorption correction

Max. and min. transmission

Refinement method

Data / restraints / parameters

Goodness-of-fit on $\mathrm{F}^{2}$

Final $\mathrm{R}$ indices [I $>2 \operatorname{sigma}(\mathrm{I})]$

$\mathrm{R}$ indices (all data)

Extinction coefficient

Largest diff. peak and hole
2302.27(9) $\AA^{3}$

4

$1.441 \mathrm{Mg} / \mathrm{m}^{3}$

$0.268 \mathrm{~mm}^{-1}$

1040

$0.177 \times 0.125 \times 0.100 \mathrm{~mm}^{3}$

2.063 to $26.402^{\circ}$.

$-11<=\mathrm{h}<=11,-24<=\mathrm{k}<=24,-16<=\mathrm{l}<=16$

65549

$4708[\mathrm{R}(\mathrm{int})=0.0386]$

$99.9 \%$

Semi-empirical from equivalents

0.7454 and 0.6899

Full-matrix least-squares on $\mathrm{F}^{2}$

4708 / 0 / 322

1.042

$\mathrm{R} 1=0.0303, \mathrm{wR} 2=0.0744$

$\mathrm{R} 1=0.0347, \mathrm{wR} 2=0.0775$

$0.0149(7)$

0.308 and -0.240 e. $\AA^{-3}$ 
(a)

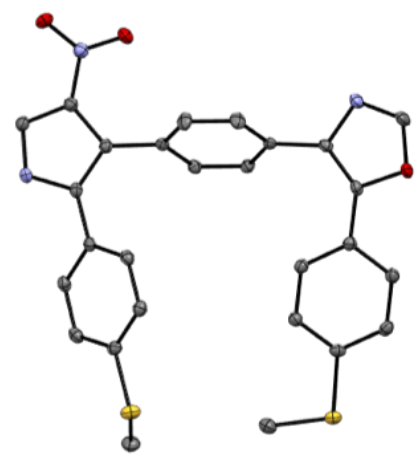

(c)

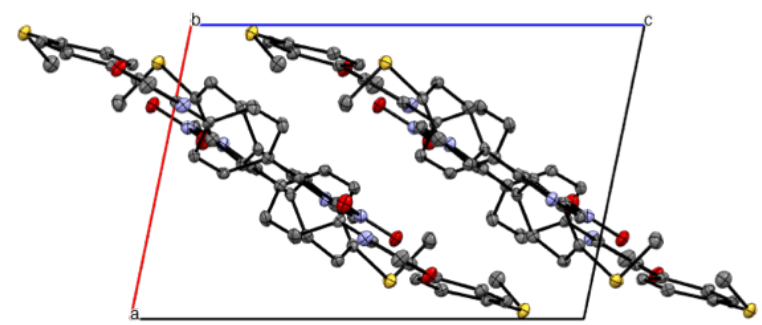

(b)

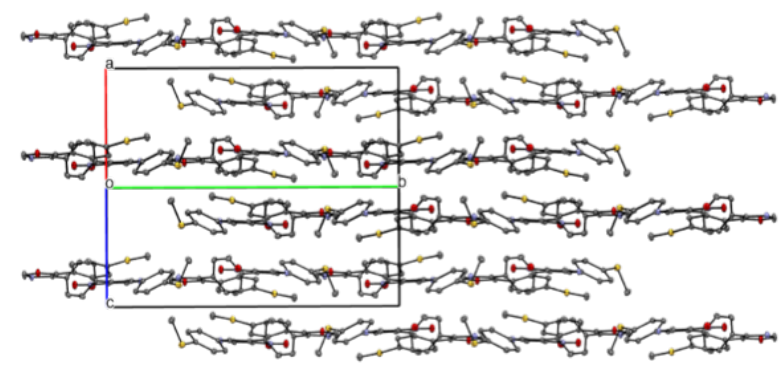

(d)

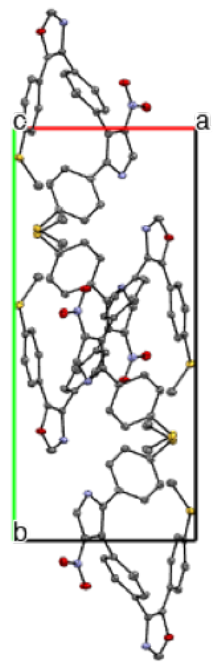

Figure S82. Selected images of POPRP with hydrogens omitted for clarity. (a) Single molecule ellipsoid view of POPRP. (b) Molecule packing along the b-axis. (c) Unit cell viewed along the b-axis. (d) Unit cell viewed along the c-axis.

\subsection{Single Crystal Data of PRPOP (crystal obtained from a solution mixture of $\mathrm{CHCl}_{3} / \mathrm{n}$ - Hexane)}

Table S3. Crystal data and structure refinement for PRPOP.

Identification code

Empirical formula

Formula weight

Temperature

Wavelength

Crystal system

Space group

Unit cell dimensions

Volume dd43ssa

C28 H22 Cl3 N3 O3 S2

618.95

100(2) K

$0.71073 \AA$

Monoclinic

$\mathrm{P} 21 / \mathrm{n}$

$\mathrm{a}=8.0539(3) \AA \quad \mathrm{a}=90^{\circ}$.

$\mathrm{b}=22.4096(7) \AA$

$b=95.7110(6)^{\circ}$.

$\mathrm{c}=15.3897(5) \AA$

2763.82(16) $\AA^{3}$ $\mathrm{g}=90^{\circ}$. 
$\mathrm{Z}$

Density (calculated)

Absorption coefficient

$\mathrm{F}(000)$

Crystal size

Theta range for data collection

Index ranges

Reflections collected

Independent reflections

Completeness to theta $=25.242^{\circ}$

Absorption correction

Max. and min. transmission

Refinement method

Data / restraints / parameters

Goodness-of-fit on $\mathrm{F}^{2}$

Final $\mathrm{R}$ indices [I $>2 \operatorname{sigma}(\mathrm{I})]$

$\mathrm{R}$ indices (all data)

Extinction coefficient

Largest diff. peak and hole
4

$1.488 \mathrm{Mg} / \mathrm{m}^{3}$

$0.520 \mathrm{~mm}^{-1}$

1272

$0.455 \times 0.231 \times 0.124 \mathrm{~mm}^{3}$

2.252 to $28.339^{\circ}$.

$-10<=\mathrm{h}<=10,-29<=\mathrm{k}<=29,-20<=\mathrm{l}<=20$

116543

$6886[\mathrm{R}(\mathrm{int})=0.0379]$

$99.9 \%$

Integration

0.9503 and 0.8205

Full-matrix least-squares on $\mathrm{F}^{2}$

6886 / 108 / 395

1.029

$\mathrm{R} 1=0.0368, \mathrm{wR} 2=0.0880$

$\mathrm{R} 1=0.0423, \mathrm{wR} 2=0.0917$

$0.0120(5)$

0.805 and -0.475 e. $\AA^{-3}$ 
(a)

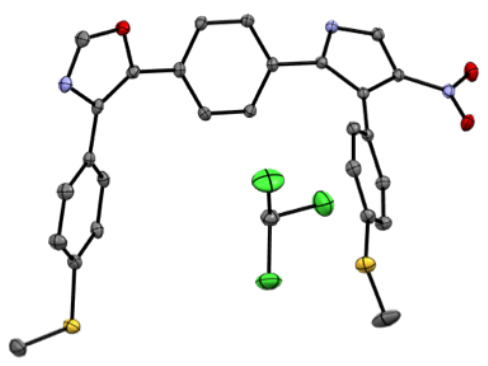

(c)

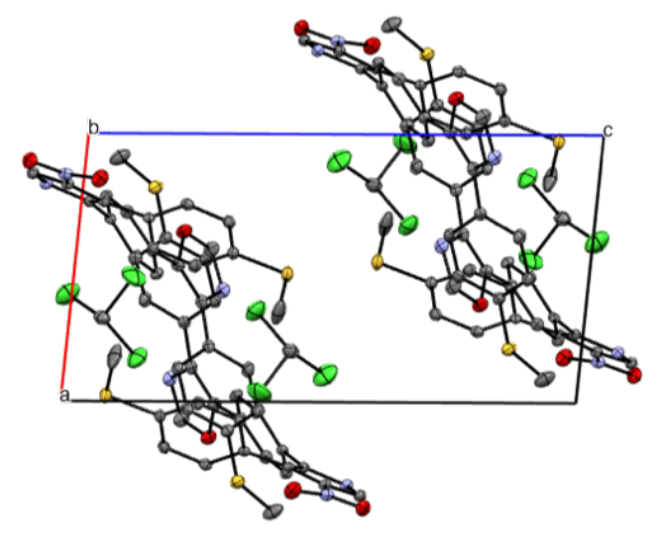

(b)

(d)
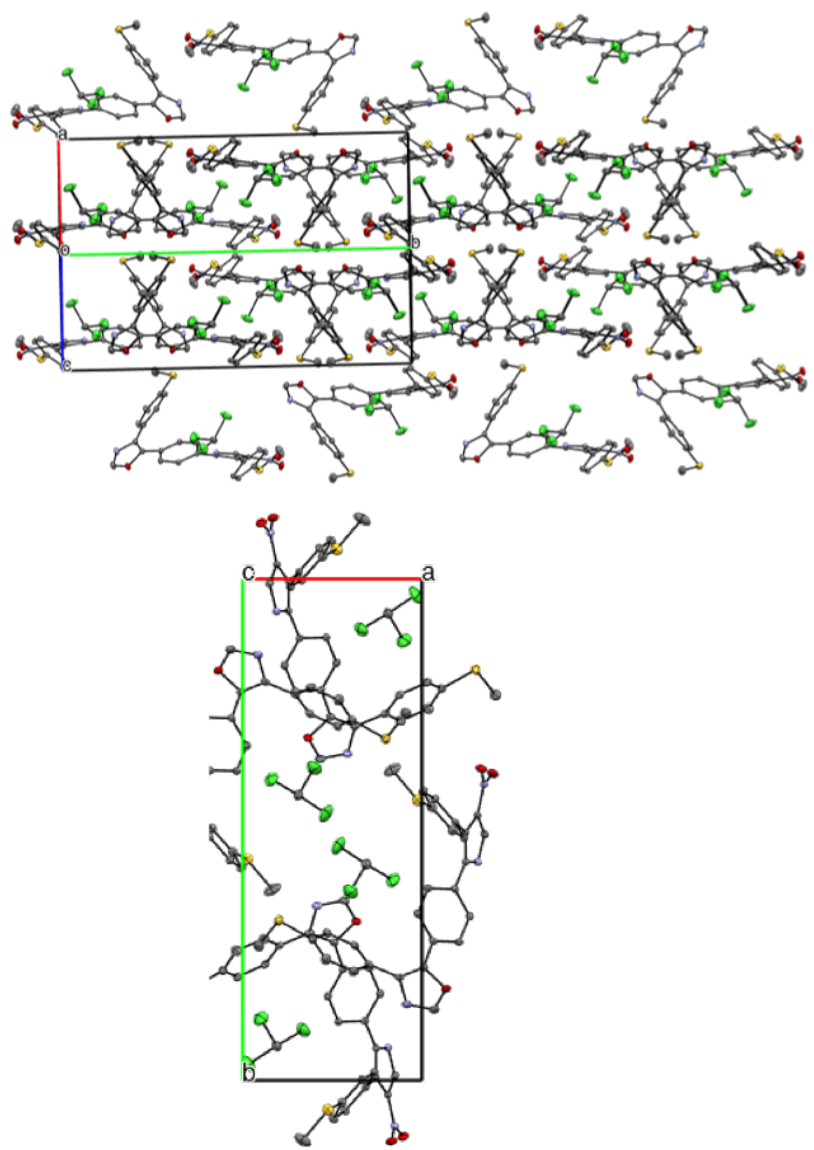

Figure S83. Selected images of PRPOP with hydrogens omitted for clarity. (a) Single molecule ellipsoid view of PRPOP. (b) Molecule packing along the b-axis. (c) Unit cell viewed along the b-axis. (d) Unit cell viewed along the c-axis.

\subsection{Single Crystal Data of PRPOP (crystal obtained from a solution mixture of EtOAc/n- Hexane)}

Table S4. Crystal data and structure refinement for PRPOP.

Identification code

Empirical formula

Formula weight

Temperature

Wavelength

Crystal system

Space group

Unit cell dimensions

Volume dd55ssa

C27 H21 N3 O3 S2

499.59

100(2) K

$0.71073 \AA$

Monoclinic

$\mathrm{P} 21 / \mathrm{n}$

$\mathrm{a}=6.6052(2) \AA \quad \mathrm{a}=90^{\circ}$.

$\mathrm{b}=26.8681(8) \AA \quad \mathrm{b}=91.3013(9)^{\circ}$.

$\mathrm{c}=13.2804(4) \AA \quad \mathrm{g}=90^{\circ}$.

2356.25(12) $\AA^{3}$ 
$\mathrm{Z}$

Density (calculated)

Absorption coefficient

$\mathrm{F}(000)$

Crystal size

Theta range for data collection

Index ranges

Reflections collected

Independent reflections

Completeness to theta $=25.242^{\circ}$

Absorption correction

Max. and min. transmission

Refinement method

Data / restraints / parameters

Goodness-of-fit on $\mathrm{F}^{2}$

Final $\mathrm{R}$ indices [I $>2 \operatorname{sigma}(\mathrm{I})]$

$\mathrm{R}$ indices (all data)

Extinction coefficient

Largest diff. peak and hole
4

$1.408 \mathrm{Mg} / \mathrm{m}^{3}$

$0.262 \mathrm{~mm}^{-1}$

1040

$0.210 \times 0.137 \times 0.084 \mathrm{~mm}^{3}$

2.743 to $28.296^{\circ}$.

$-8<=\mathrm{h}<=8,-35<=\mathrm{k}<=35,-17<=\mathrm{l}<=17$

46182

$5831[\mathrm{R}(\mathrm{int})=0.0549]$

$99.9 \%$

Integration

0.9871 and 0.9481

Full-matrix least-squares on $\mathrm{F}^{2}$

$5831 / 0 / 322$

1.047

$\mathrm{R} 1=0.0428, \mathrm{wR} 2=0.0811$

$\mathrm{R} 1=0.0624, \mathrm{wR} 2=0.0880$

$0.0073(4)$

0.353 and -0.332 e. $\AA^{-3}$ 
(a)

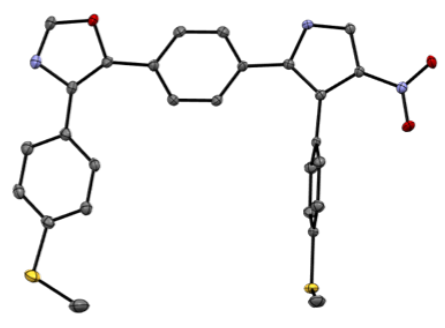

(c)

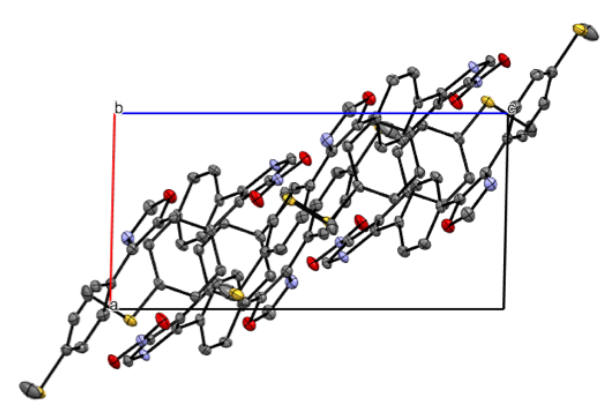

(b)

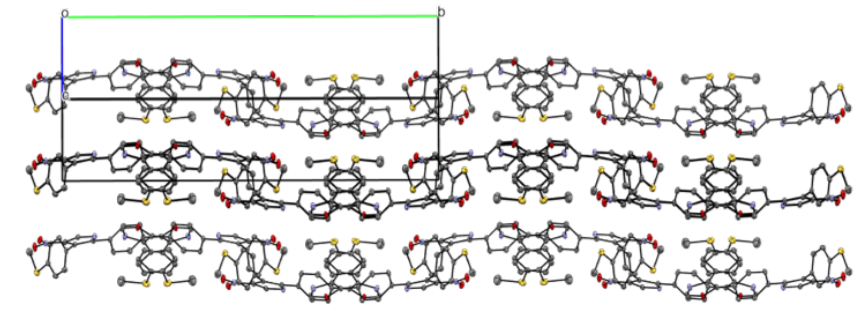

(d)

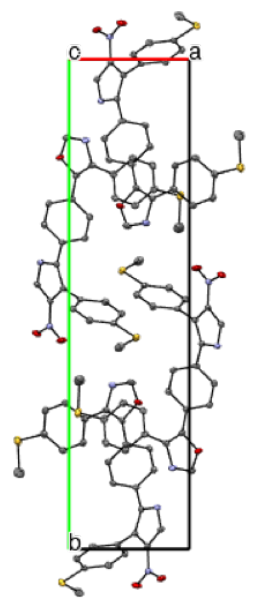

Figure S84. Selected images of PRPOP with hydrogens omitted for clarity. (a) Single molecule ellipsoid view of PRPOP. (b) Molecule packing along the b-axis. (c) Unit cell viewed along the b-axis. (d) Unit cell viewed along the c-axis. 


\section{Single Molecule Conductance Measurements}

\subsection{D Histograms of Sequence-defined Oligomers}

a)

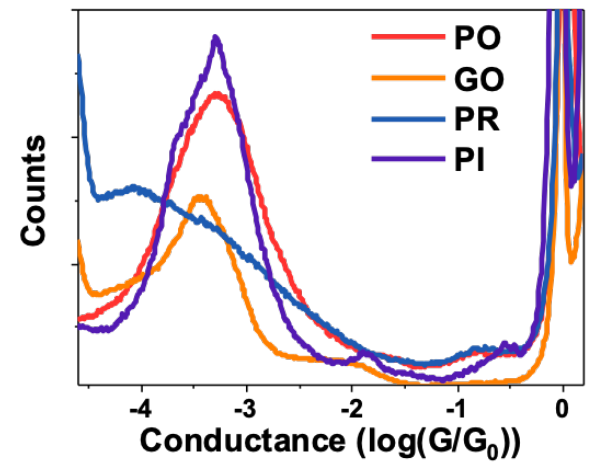

b)

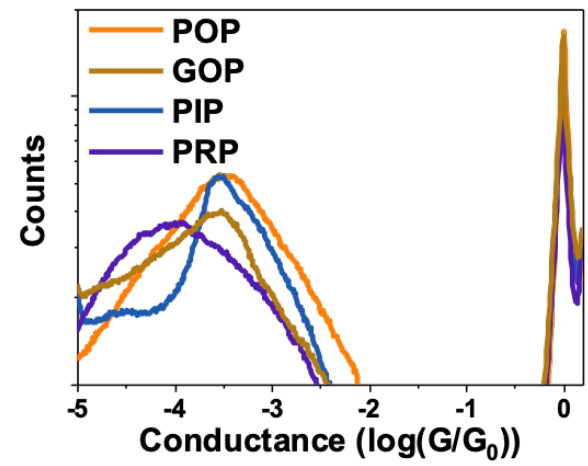

Figure S85. 1D conductance histograms of: (a) dimers and (b) trimers at $0.25 \mathrm{~V}$ bias voltage.
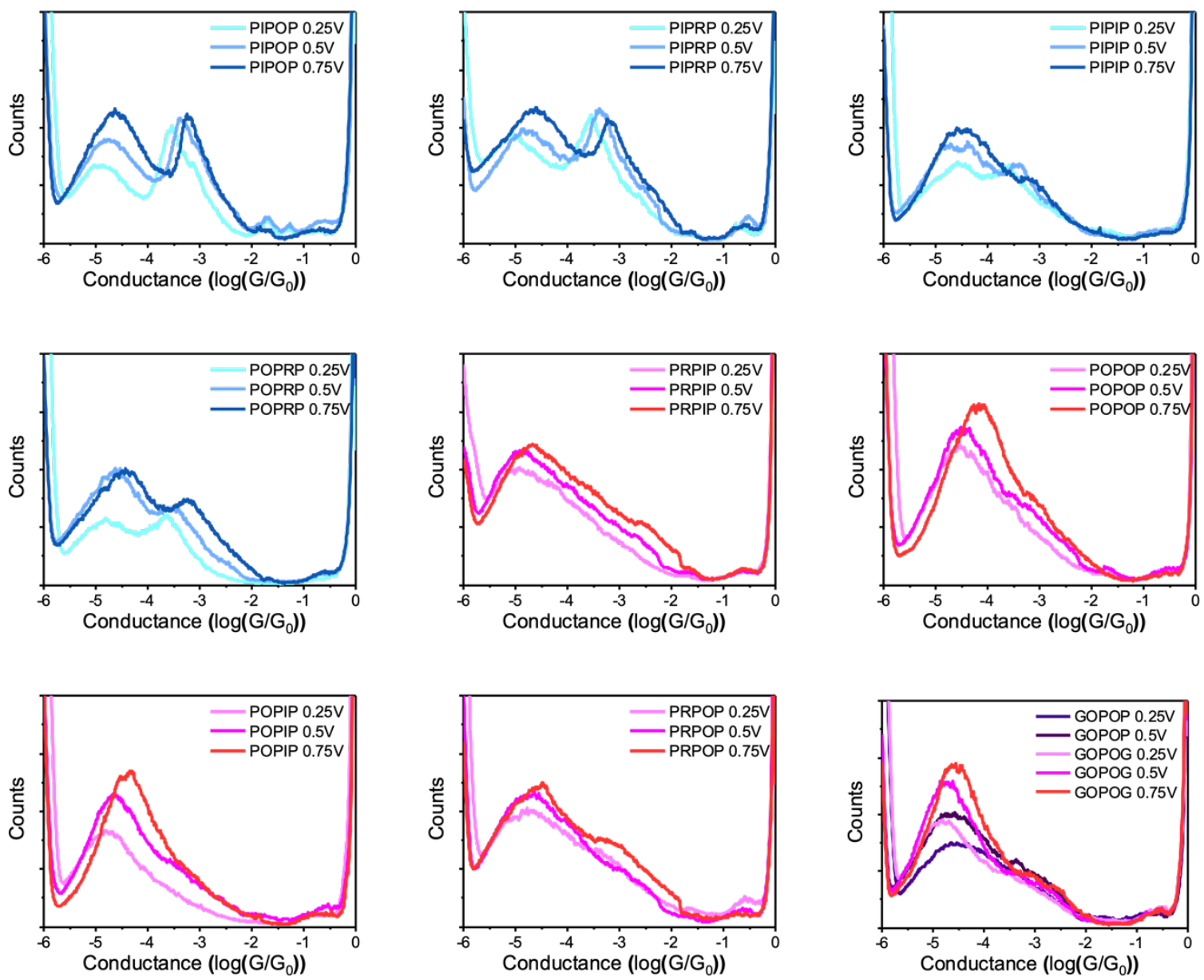

Figure S86. 1D conductance histograms of pentamers at different bias voltages $(0.25 \mathrm{~V}, 0.50 \mathrm{~V}$, $0.75 \mathrm{~V})$. 


\subsection{D Histogram of Sequence-defined Oligomers}

a)

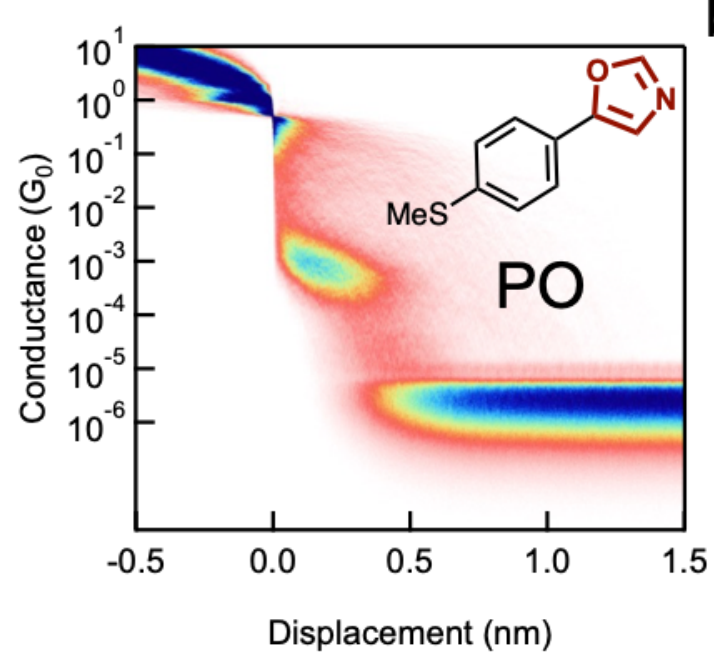

c)

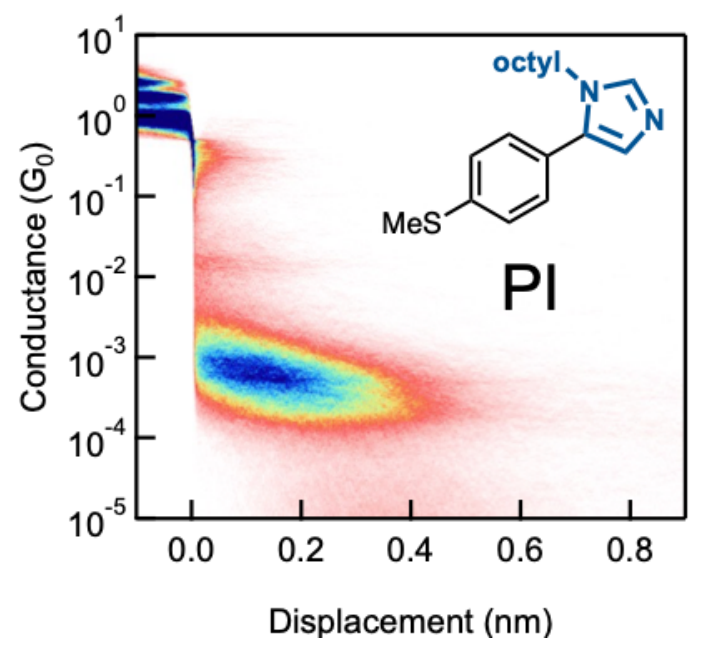

b)

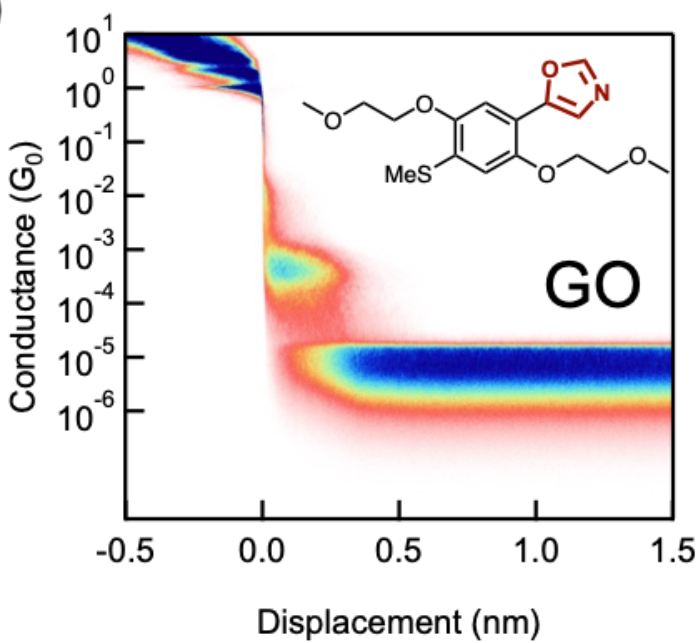

d)

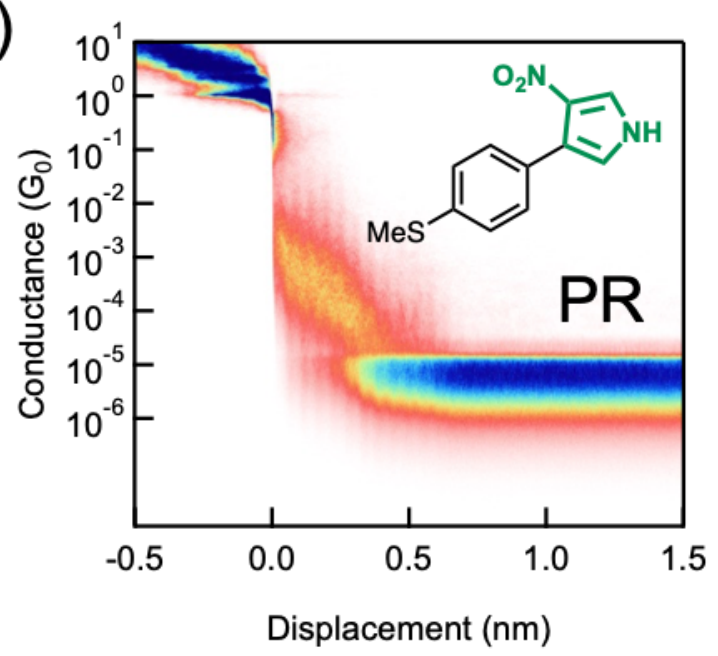

Figure S87. 2D histogram of dimers a) PO; b) GO; c) PI; d) PR at 0.25 V bias voltage. 
a)

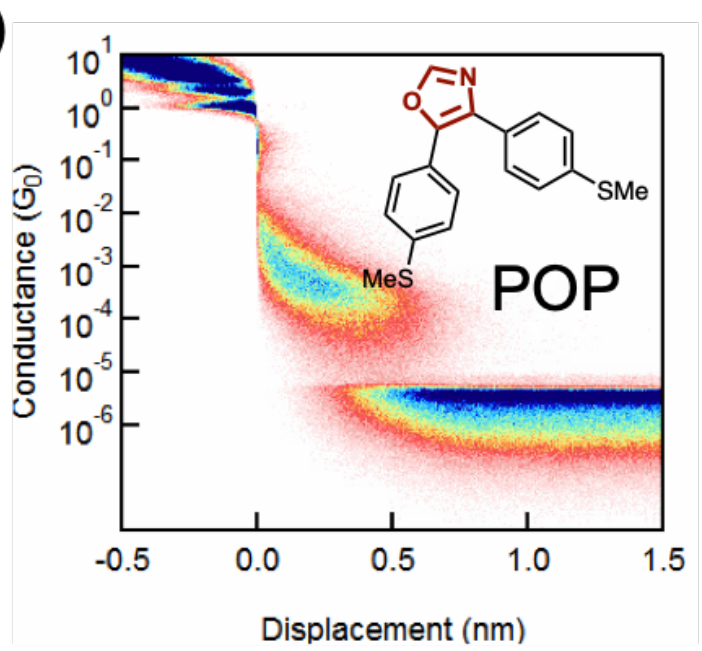

c)

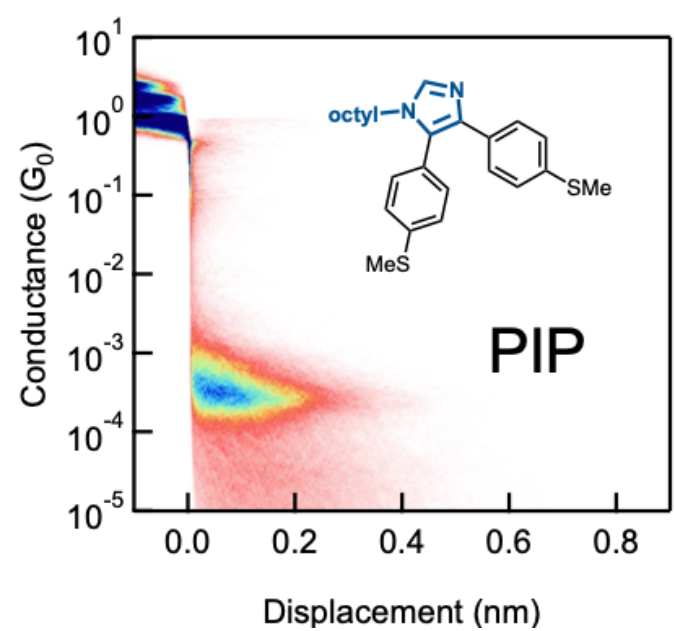

b)

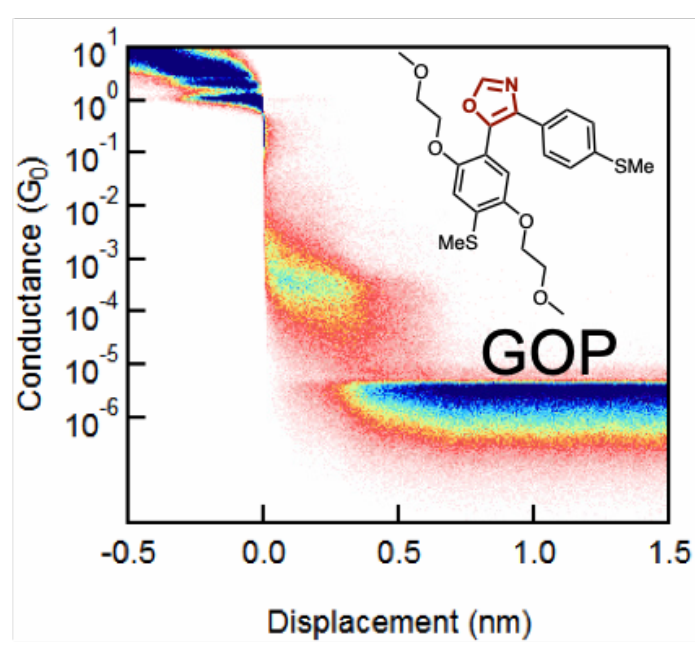

d)

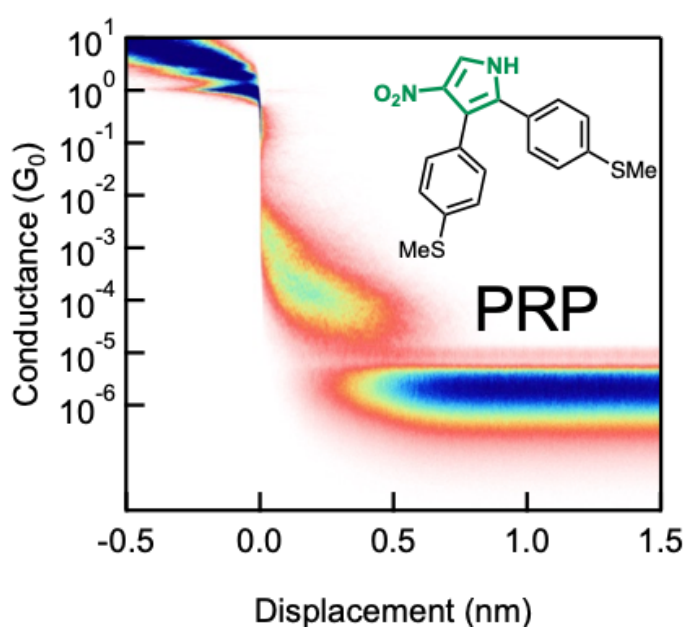

Figure S88. 2D histogram of Trimers a) POP; b) GOP; c) PIP; d) PRP at 0.25 V bias voltage.
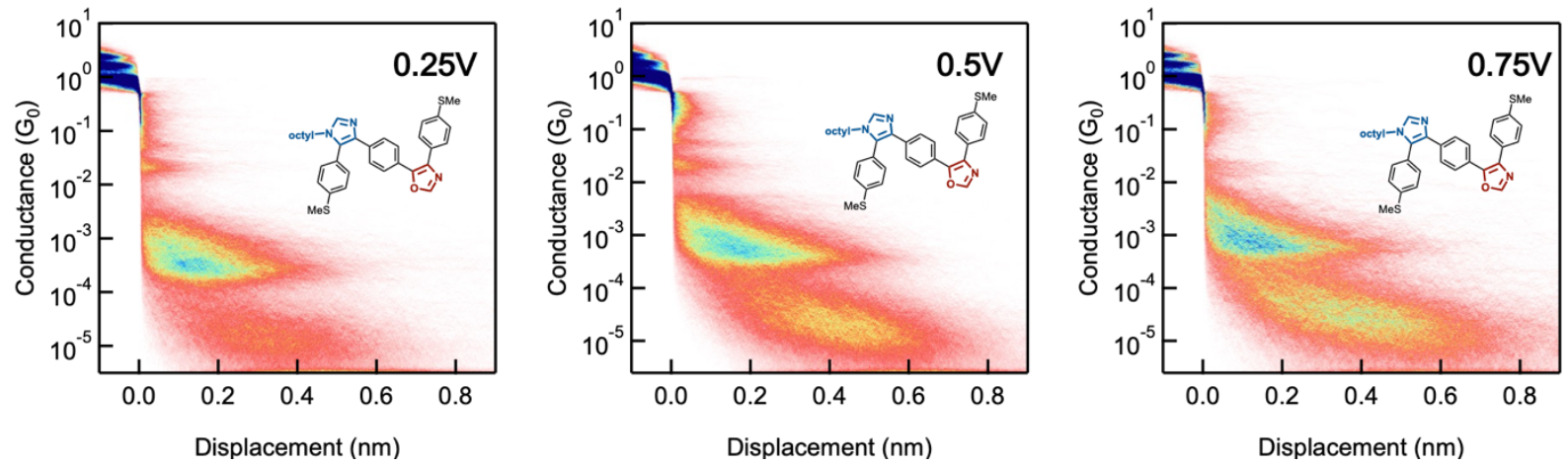

Figure S89. 2D histogram of PIPOP at different bias voltages $(0.25 \mathrm{~V}, 0.50 \mathrm{~V}, 0.75 \mathrm{~V})$. 

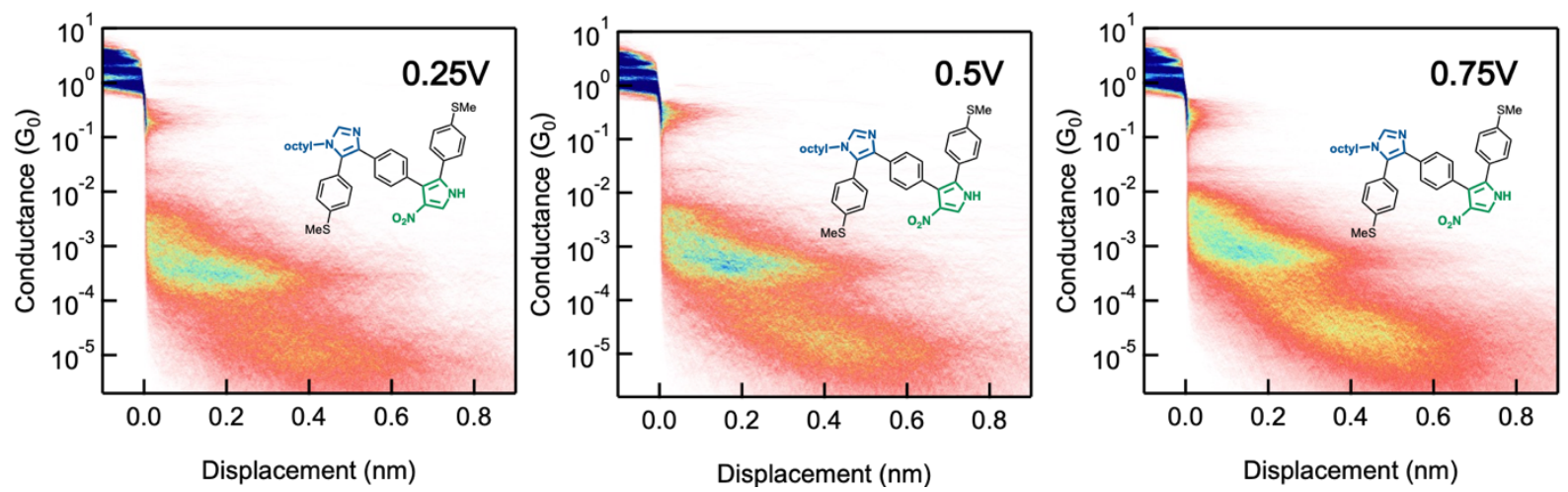

Figure S90. 2D histogram of PIPRP at different bias voltages $(0.25 \mathrm{~V}, 0.50 \mathrm{~V}, 0.75 \mathrm{~V})$.
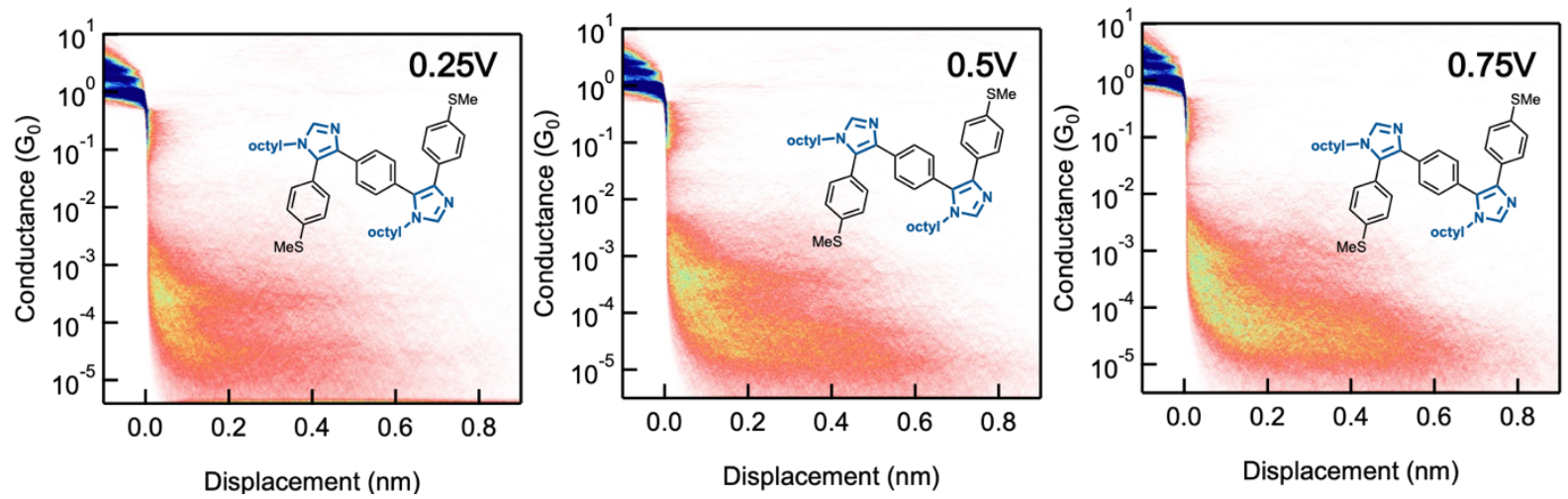

Figure S91. 2D histogram of PIPIP at different bias voltages $(0.25 \mathrm{~V}, 0.50 \mathrm{~V}, 0.75 \mathrm{~V})$.
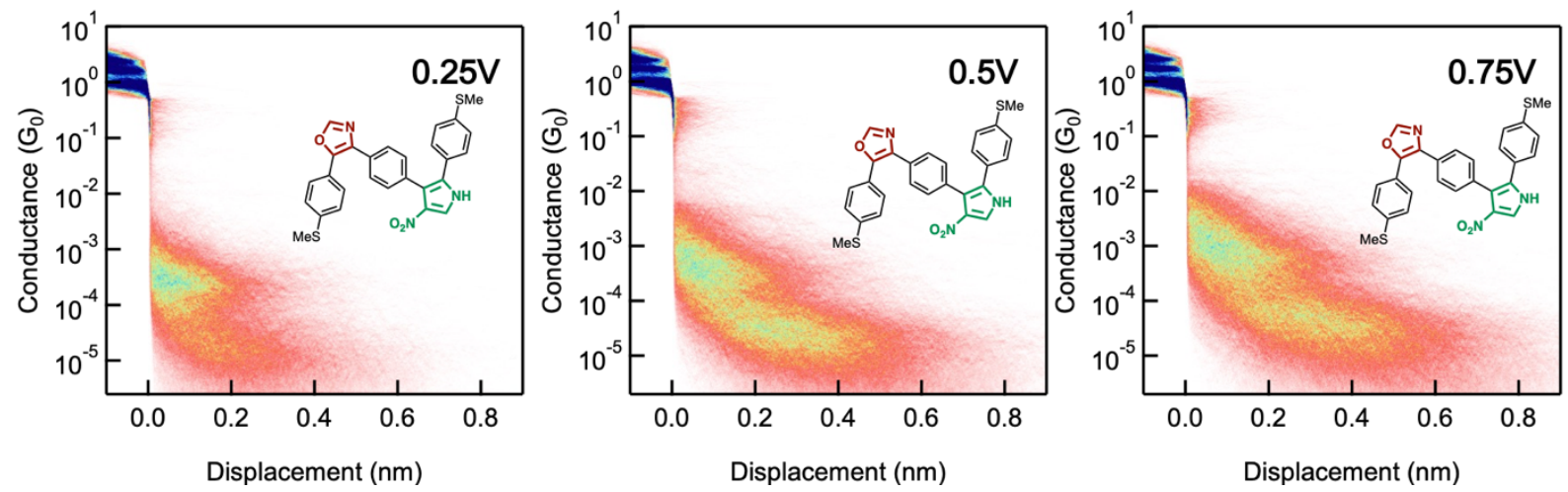

Figure S92. 2D histogram of POPRP at different bias voltages $(0.25 \mathrm{~V}, 0.50 \mathrm{~V}, 0.75 \mathrm{~V})$. 

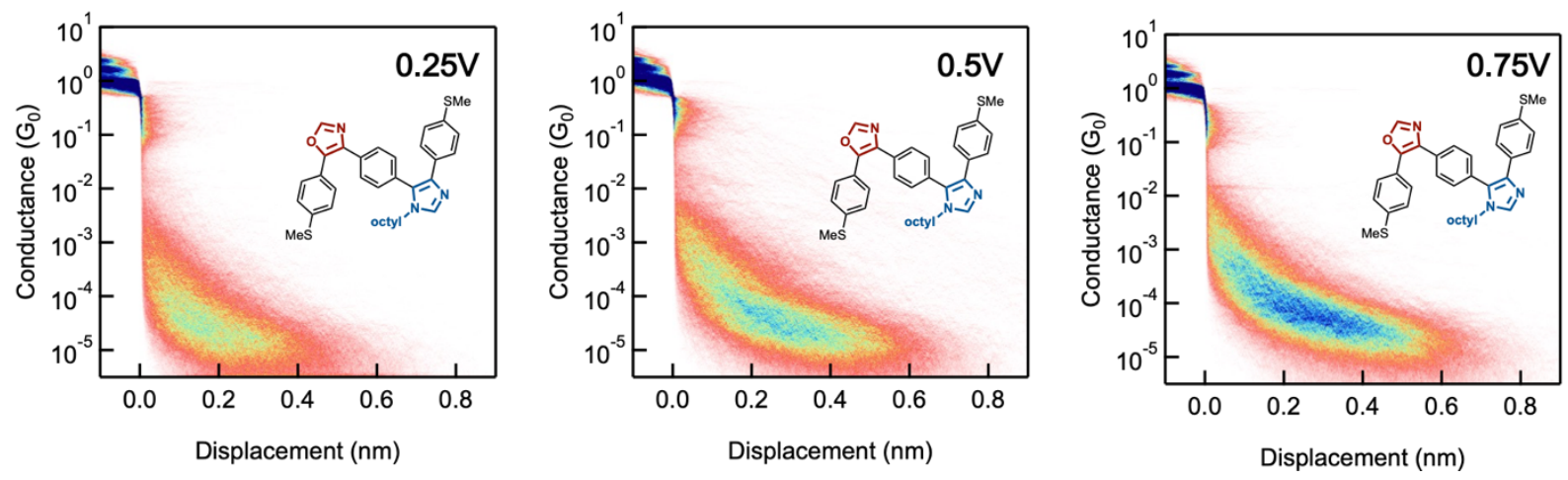

Figure S93. 2D histogram of POPIP at different bias voltages $(0.25 \mathrm{~V}, 0.50 \mathrm{~V}, 0.75 \mathrm{~V})$.
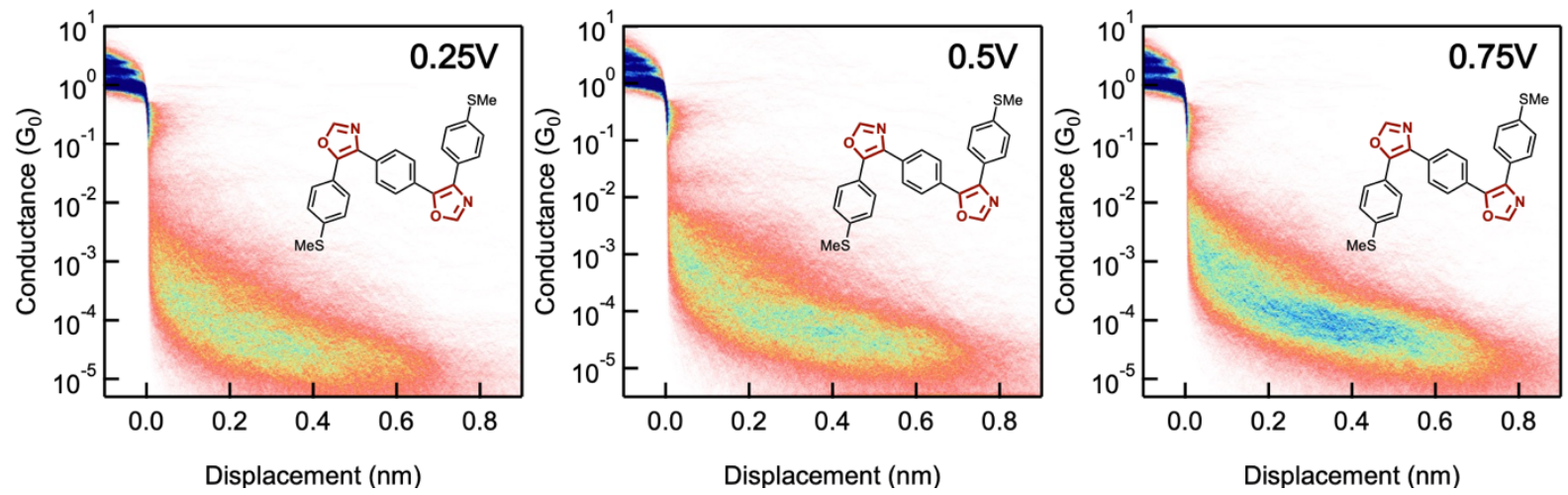

Figure S94. 2D histogram of POPOP at different bias voltages $(0.25 \mathrm{~V}, 0.50 \mathrm{~V}, 0.75 \mathrm{~V})$.
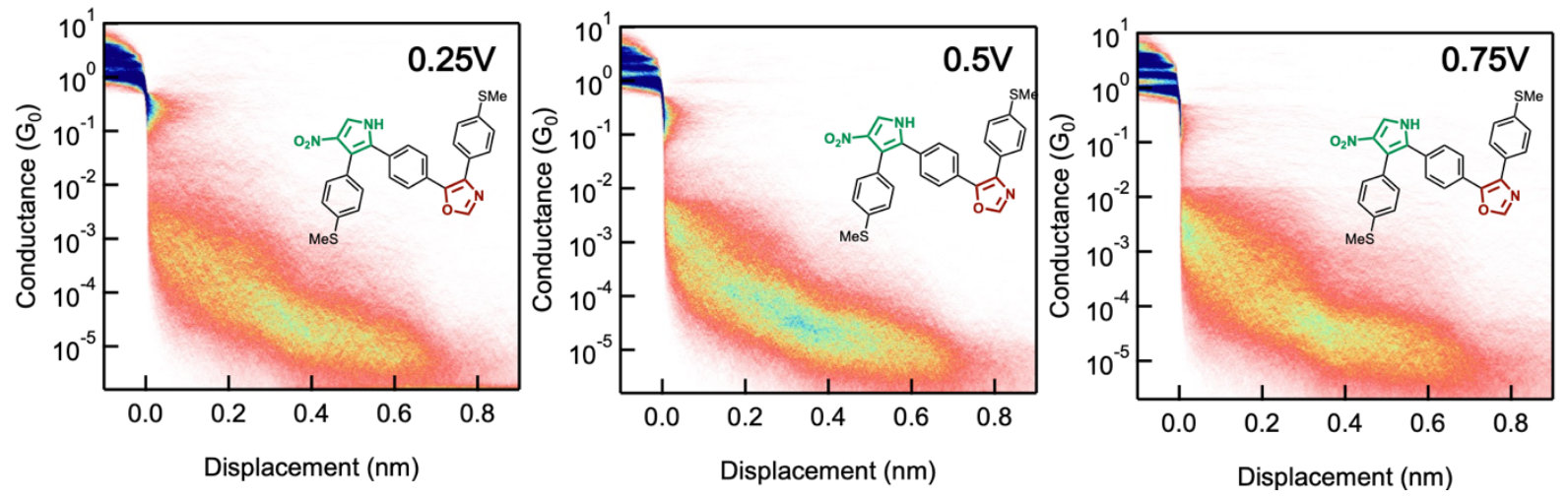

Figure S95. 2D histogram of PRPOP at different bias voltages $(0.25 \mathrm{~V}, 0.50 \mathrm{~V}, 0.75 \mathrm{~V})$. 

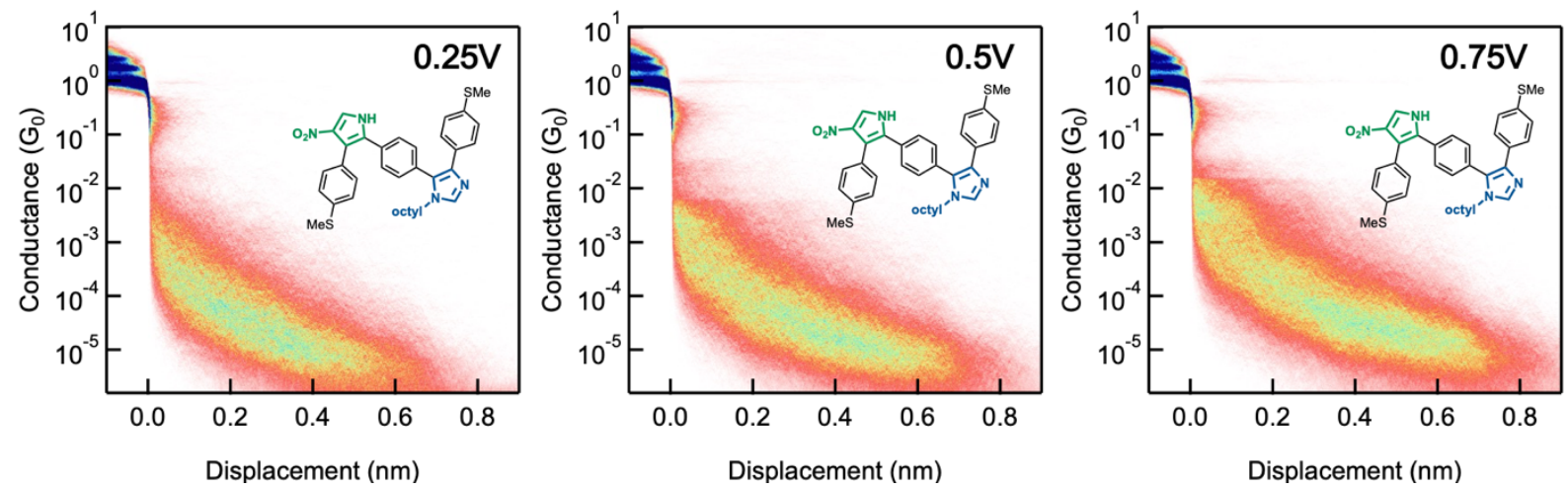

Figure S96. 2D histogram of PRPIP at different bias voltages $(0.25 \mathrm{~V}, 0.50 \mathrm{~V}, 0.75 \mathrm{~V})$.
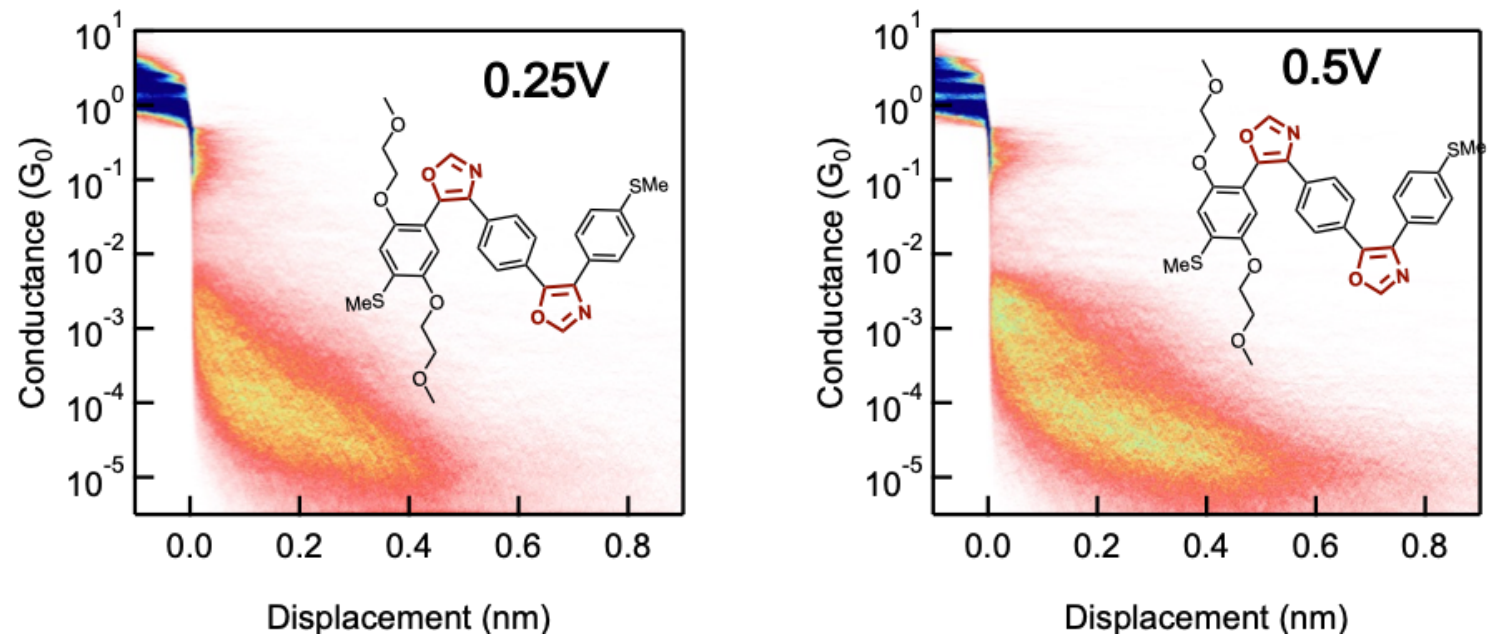

Figure S97. 2D histogram of GOPOP at different bias voltages $(0.25 \mathrm{~V}, 0.50 \mathrm{~V})$.
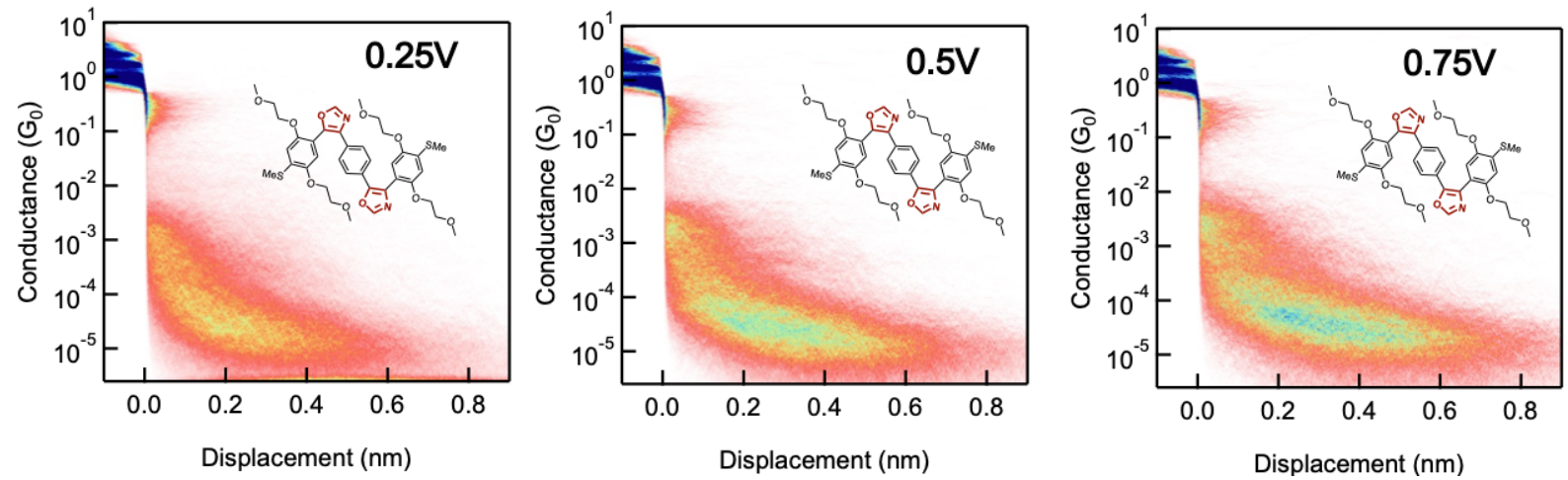

Figure S98. 2D histogram of GOPOG at different bias voltages $(0.25 \mathrm{~V}, 0.50 \mathrm{~V}, 0.75 \mathrm{~V})$. 

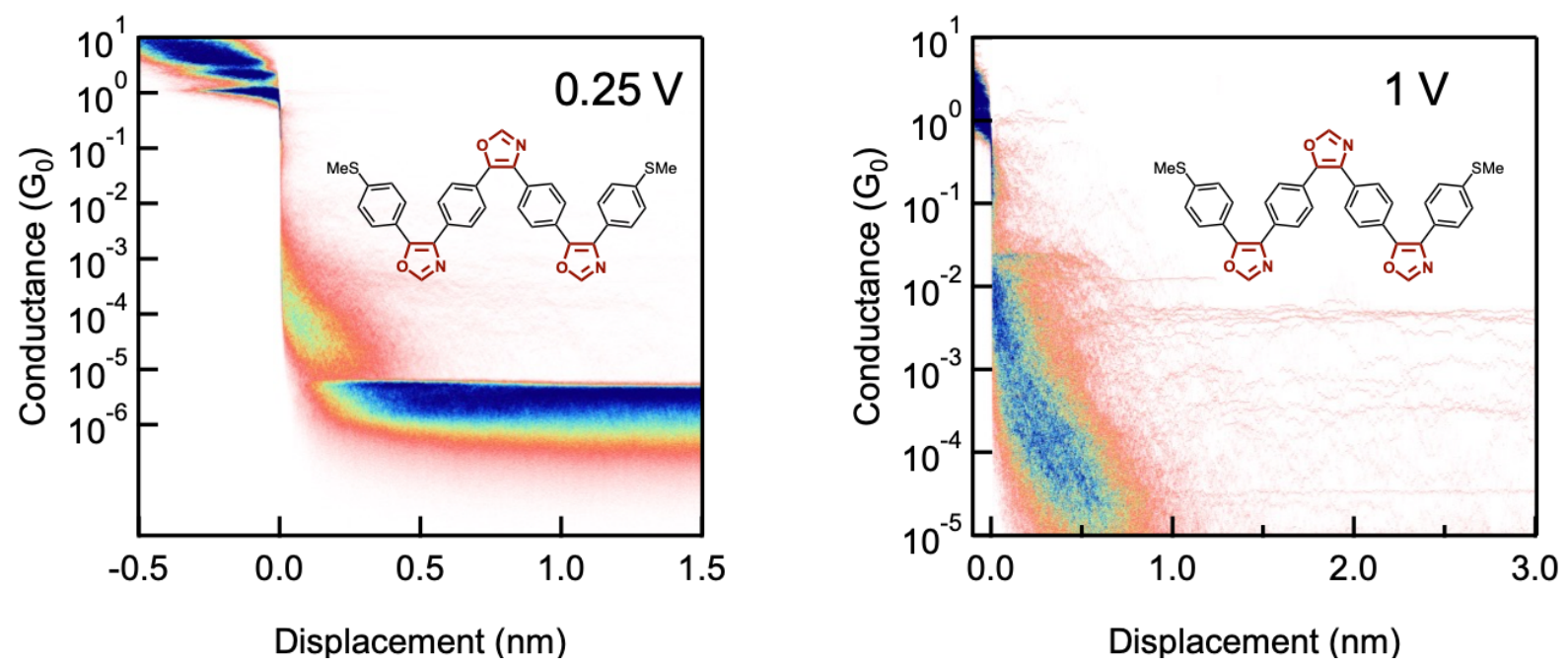

Figure S99. 2D histogram of POPOPOP at different bias voltages $(0.25 \mathrm{~V}, 0.10 \mathrm{~V})$.

\subsection{D and 2D Histogram of Control Compounds}

\subsubsection{Control Compounds for PIPOP}
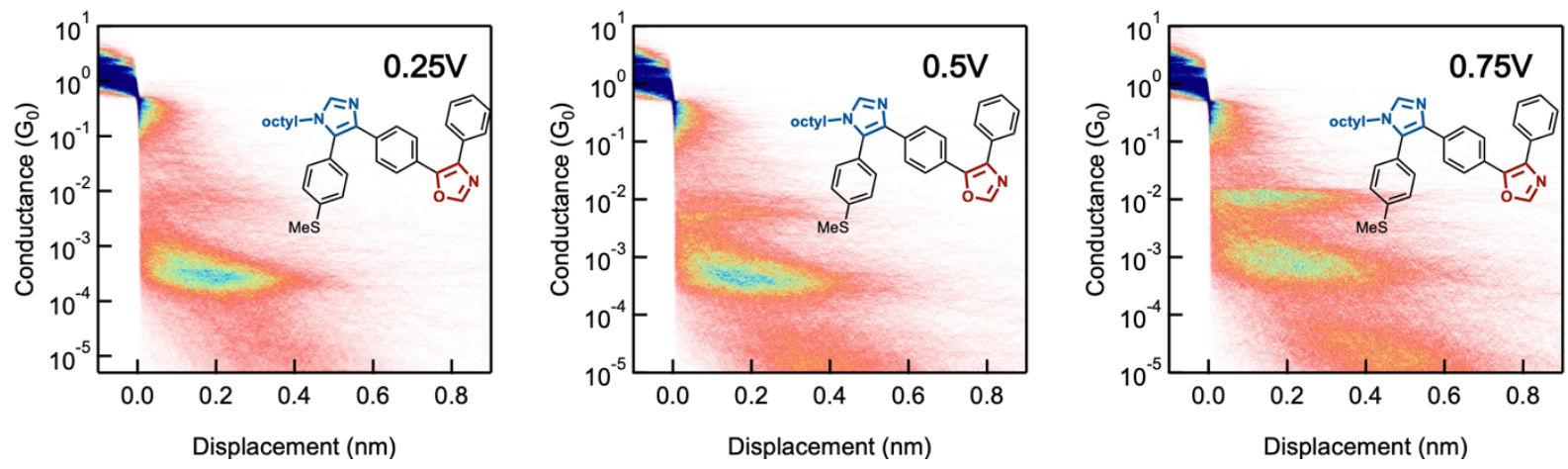

Figure S100. 2D histogram of S-PIPOP-H at different bias voltages $(0.25 \mathrm{~V}, 0.50 \mathrm{~V}, 0.75 \mathrm{~V})$.
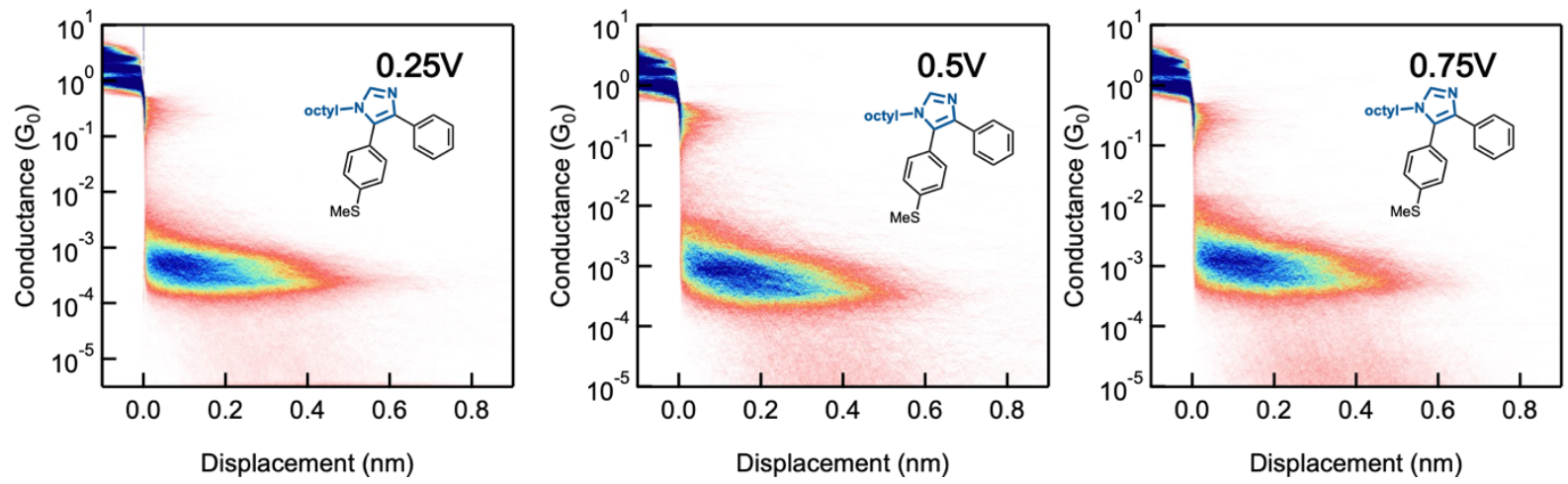

Figure S101. 2D histogram of S-PIP-H at different bias voltages $(0.25 \mathrm{~V}, 0.50 \mathrm{~V}, 0.75 \mathrm{~V})$. 

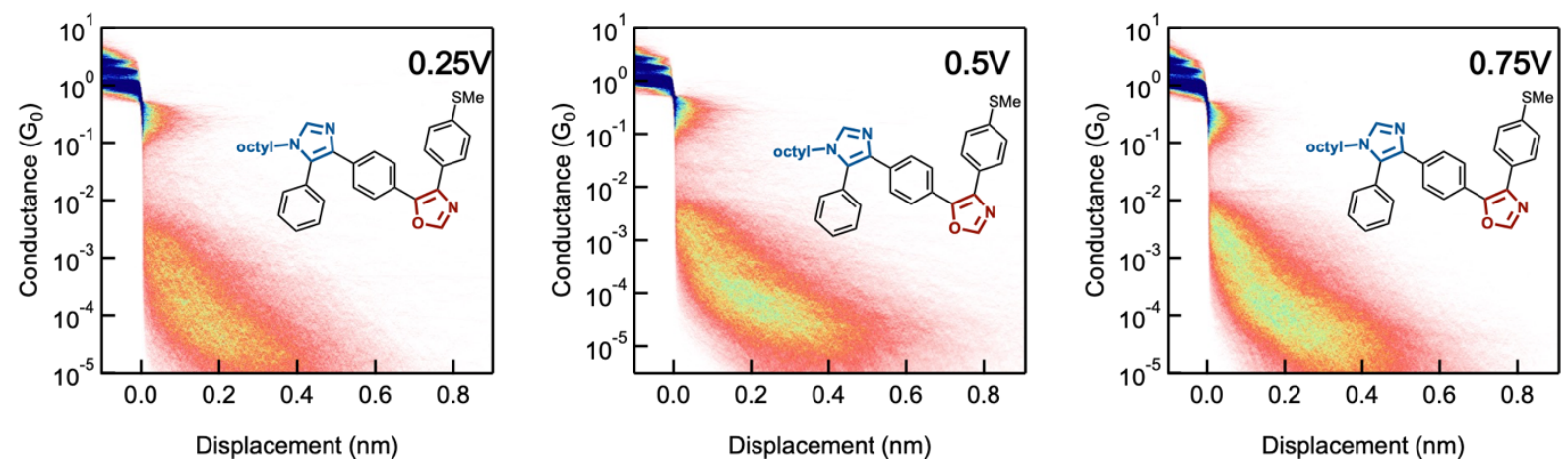

Figure S102. 2D histogram of H-PIPOP-S at different bias voltages $(0.25 \mathrm{~V}, 0.50 \mathrm{~V}, 0.75 \mathrm{~V})$.
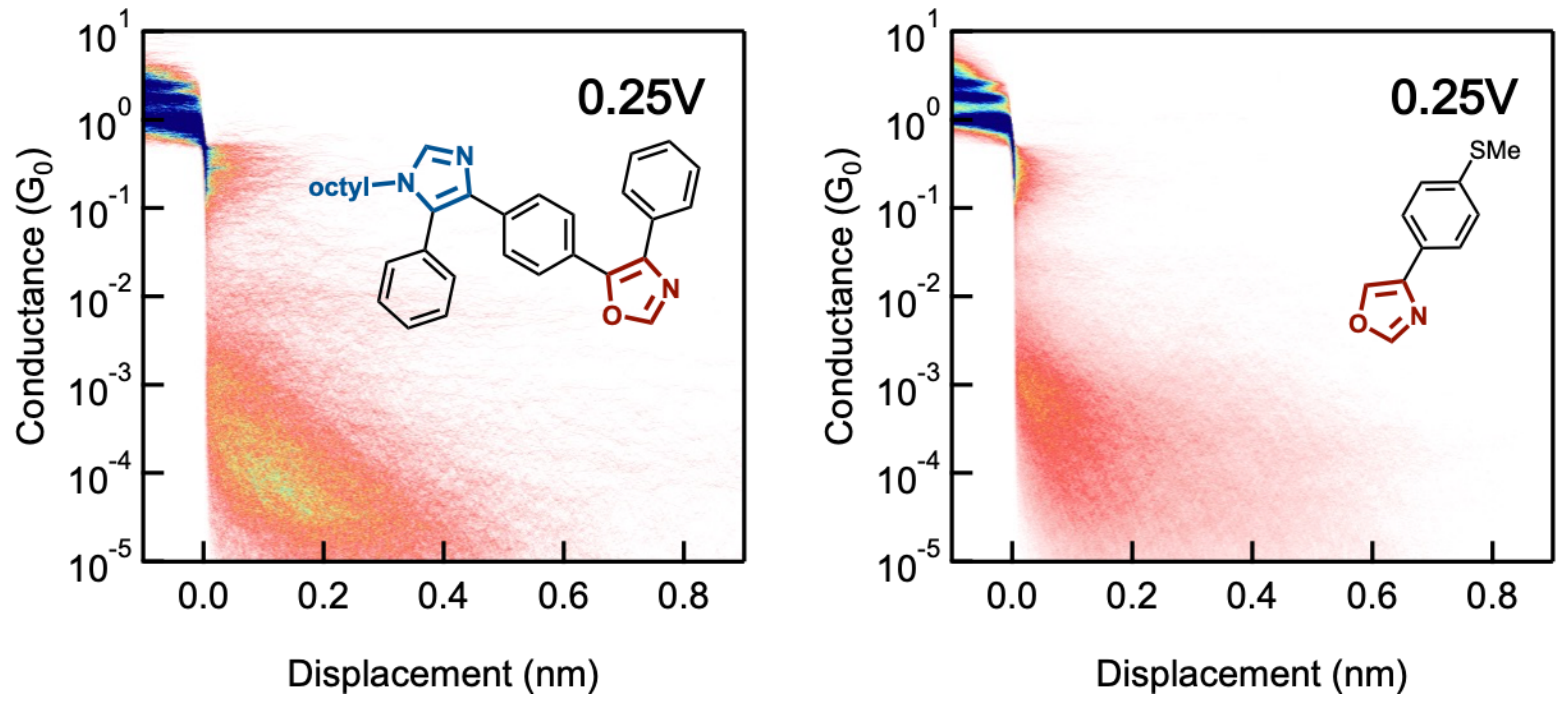

Figure S103. 2D histogram of H-PIPOP-H and OP at $0.25 \mathrm{~V}$. 


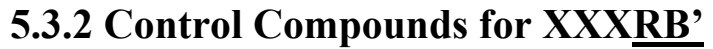
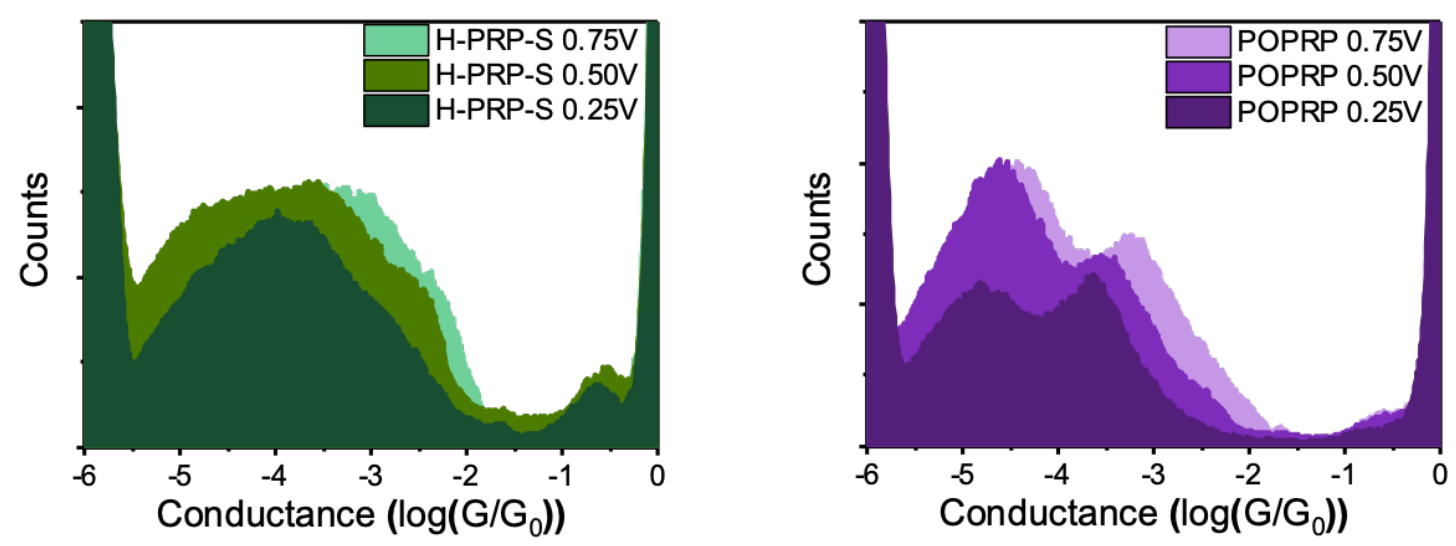

Figure S104. Comparison of 1D histogram of H-PRP-S and POPRP at different bias voltages $(0.25 \mathrm{~V}, 0.50 \mathrm{~V}, 0.75 \mathrm{~V})$.
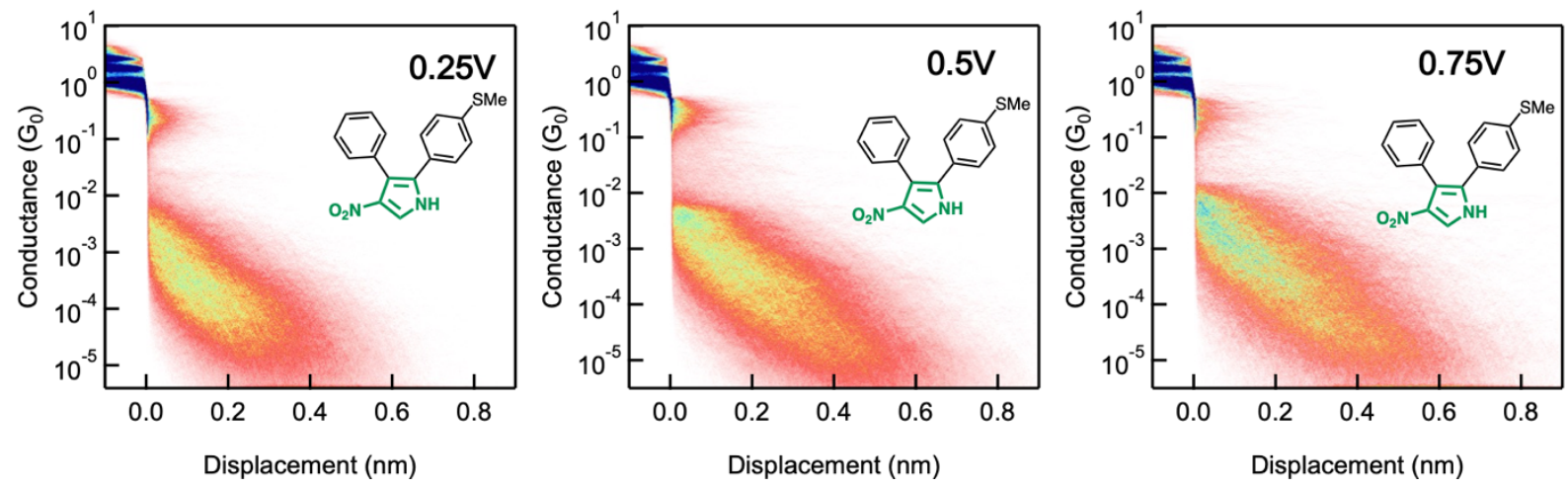

Figure S105. 2D histogram of H-PRP-S at different bias voltages $(0.25 \mathrm{~V}, 0.50 \mathrm{~V}, 0.75 \mathrm{~V})$. 


\subsection{Lorentzian Fitting Analysis for PRPIP, POPIP, GOPOG, PRPOP, GOPOP, and POPOP}

Lorentzian curve fitting analysis of 1D conductance histograms is performed using the Multiple Peak Fit Tool in Origin (v2018b). In the following analyses and plots shown in Figs. S106-S111, blue lines represent the cumulative fitting curve, whereas red lines represent the dominant peak fitting curve and green lines represent the secondary peak fitting curve. Fitting parameters are summarized in the table at the top right corner of each figure. Lorentzian function has a general form: $y=y_{0}+\frac{2 A}{\pi} \frac{w}{4(x-x c)^{2}+w^{2}}$, where $y_{0}$ is the offset along the y-axis, $A$ is the peak integration area, $w$ is the full width at half-maximum (FWHM), and $x_{c}$ is the peak position.

Based on this analysis, we determine the relative contribution from the primary peak using the ratio of the integration areas under the dominant and secondary Lorentzian curves: $A_{\text {dominant }}\left(A_{\text {secondary }}+A_{\text {dominant }}\right)$. The values for the ratio $A_{\text {dominant }} /\left(A_{\text {secondary }}+A_{\text {dominant }}\right)$ for pentamers PRPIP, GOPOG, PRPOP, GOPOP, and POPOP are 0.7, 0.81, 0.90, 0.83, and 0.85, respectively. Multiple peak fitting is not applicable for POPIP (Fig. S107), as the secondary peak is essentially non-existent (the ratio $A_{\text {dominant }}\left(A_{\text {secondary }}+A_{\text {dominant }}\right)$ is 1.0$)$. From this analysis, we conclude that the secondary peaks (or weak shoulder) are relatively minor compared to the dominant peak. Overall, the behavior of these pentamers is markedly different than the other set of pentamers with two well-spaced peaks (PIPRP, PIPOP, POPRP, and PIPIP).

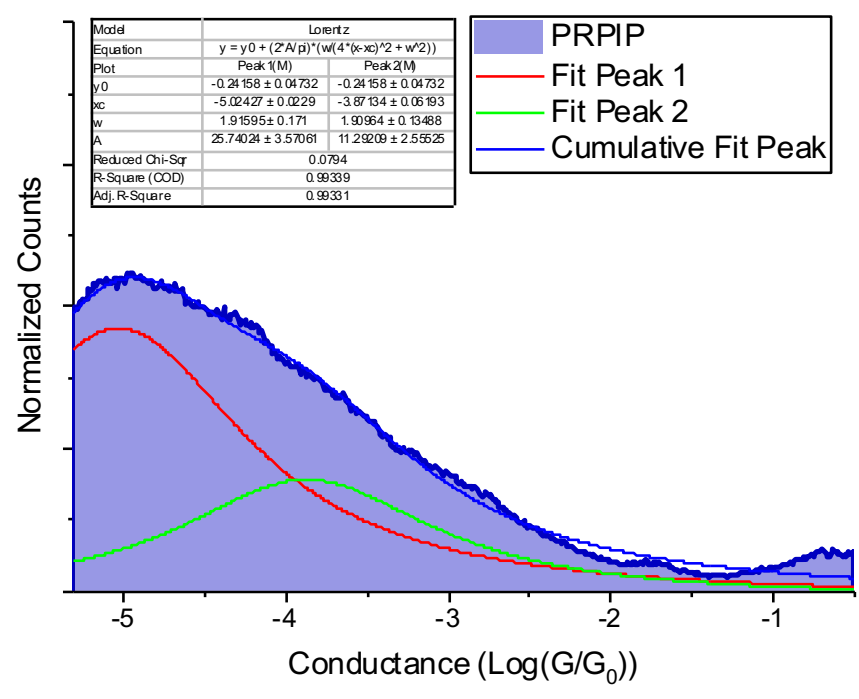

Figure S106. Multi-peak Lorentzian fitting of 1D histogram of PRPIP (bias voltage 0.25 V). 


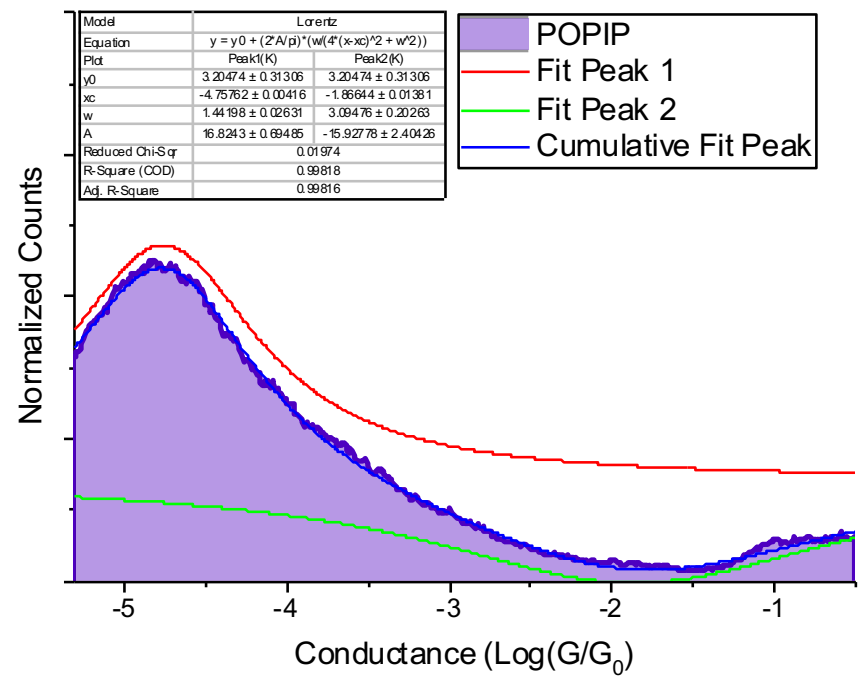

Figure S107. Multi-peak Lorentzian fitting of 1D histogram of POPIP (bias voltage 0.25 V).

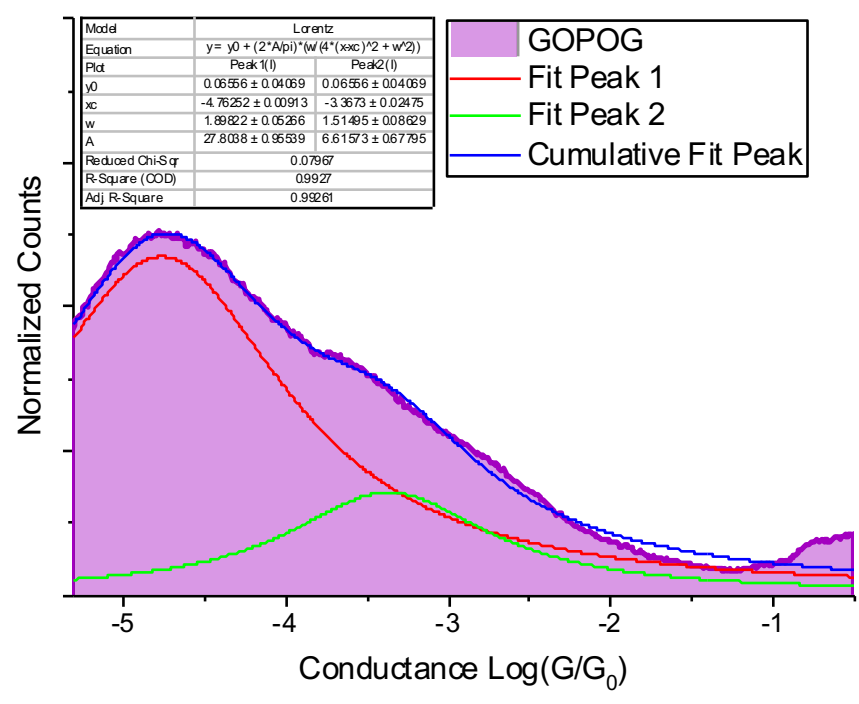

Figure S108. Multi-peak Lorentzian fitting of 1D histogram of GOPOG (bias voltage 0.25 V). 


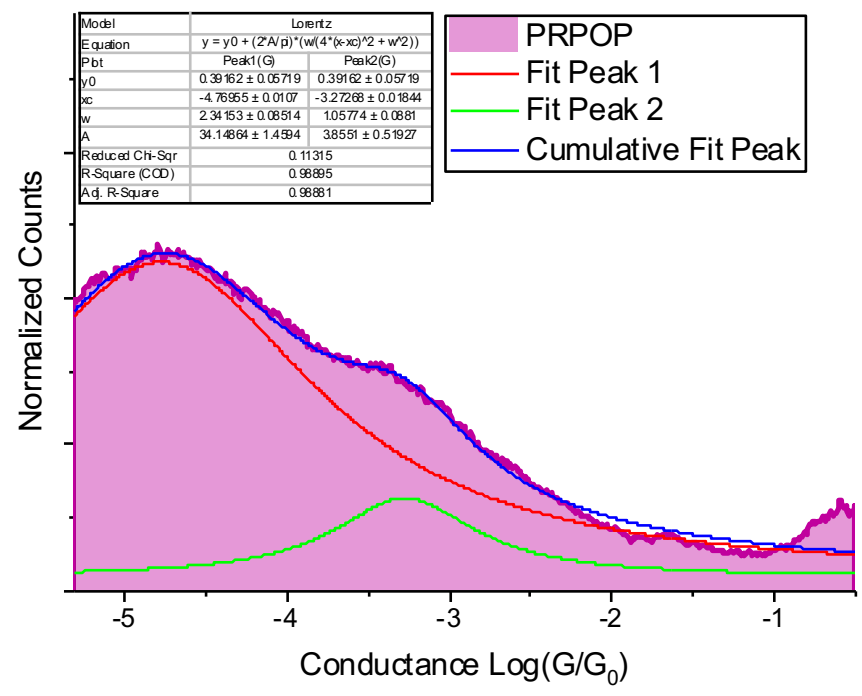

Figure S109. Multi-peak Lorentzian fitting of 1D histogram of PRPOP (bias voltage 0.25 V).

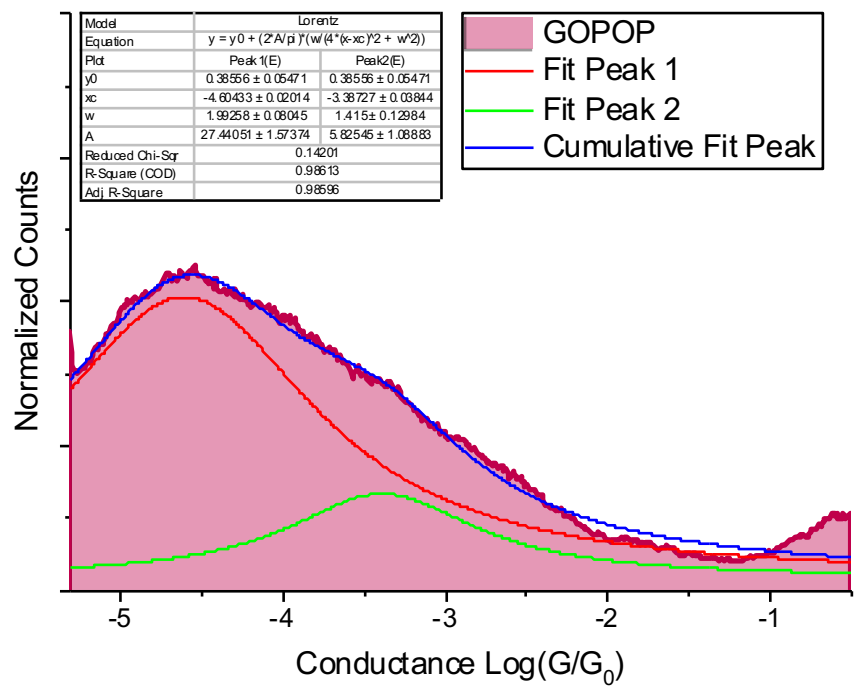

Figure S110. Multi-peak Lorentzian fitting of 1D histogram of GOPOP (bias voltage 0.25 V). 


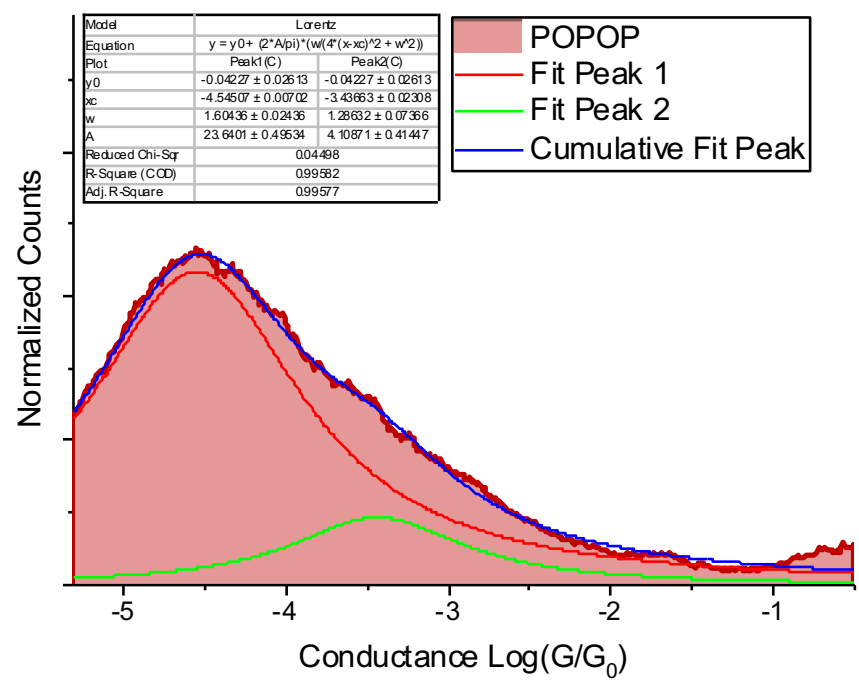

Figure S111. Multi-peak Lorentzian fitting of 1D histogram of POPOP (bias voltage 0.25 V). 


\section{Density Functional Theory (DFT) Simulations}

Table S5. Summary of single-molecule conductance of dimers and trimers

\begin{tabular}{|l|l|l|}
\hline Sequence & Conductance (STM-BJ) & Fitting Standard error \\
\hline PO & -3.31 & 0.002 \\
\hline PI & -3.56 & 0.008 \\
\hline PR & -3.96 & 0.017 \\
\hline GO & -3.56 & 0.008 \\
\hline POP & -3.97 & 0.003 \\
\hline PIP & -3.44 & 0.006 \\
\hline PRP & -3.51 & 0.003 \\
\hline GOP & -3.80 & 0.010 \\
\hline
\end{tabular}

Table S6. Summary of single-molecule conductance of pentamers

\begin{tabular}{|l|l|l|l|}
\hline Sequence & $\begin{array}{l}\text { Conductance } \\
\left(\log \left(\mathrm{G} / \mathrm{G}_{0}\right), \text { STM-BJ) }\right.\end{array}$ & $\begin{array}{l}\text { Fitting Standard } \\
\text { error }\end{array}$ & $\begin{array}{l}\text { Conductance }\left(\log \left(\mathrm{G} / \mathrm{G}_{0}\right),\right. \\
\text { DFT simulation })\end{array}$ \\
\hline PRPIP & -4.91 & 0.010 & -4.53 \\
\hline POPIP & -4.77 & 0.002 & -4.15 \\
\hline GOPOG & -4.74 & 0.004 & NA \\
\hline PRPOP & -4.72 & 0.003 & -3.36 \\
\hline GOPOP & -4.57 & 0.005 & NA \\
\hline POPOP & -4.51 & 0.004 & -3.67 \\
\hline PIPRP (High G) & -3.51 & 0.002 & NA \\
\hline PIPOP (High G) & -3.45 & 0.005 & NA \\
\hline POPRP (High G) & -3.60 & 0.003 & NA \\
\hline PIPIP (High G) & -3.57 & 0.005 & NA \\
\hline PIPRP (Low G) & -5.07 & 0.007 & -4.44 \\
\hline PIPOP (Low G) & -4.96 & 0.009 & -4.23 \\
\hline POPRP (Low G) & -4.86 & 0.004 & -4.40 \\
\hline PIPIP (Low G) & -4.61 & 0.005 & -3.62 \\
\hline
\end{tabular}

Table S7. Summary of single-molecule conductance under different bias voltage

\begin{tabular}{|l|l|l|l|}
\hline Sequence & $\begin{array}{l}\text { Conductance } \\
\left(\log \left(\mathrm{G} / \mathrm{G}_{0}\right), 0.25 \mathrm{~V}\right)\end{array}$ & $\begin{array}{l}\text { Conductance } \\
\left(\log \left(\mathrm{G} / \mathrm{G}_{0}\right), 0.50 \mathrm{~V}\right)\end{array}$ & $\begin{array}{l}\text { Conductance } \\
\left(\log \left(\mathrm{G} / \mathrm{G}_{0}\right), 0.75 \mathrm{~V}\right)\end{array}$ \\
\hline PRPIP & -4.91 & -4.85 & -4.62 \\
\hline POPIP & -4.77 & -4.63 & -4.37 \\
\hline GOPOG & -4.74 & -4.68 & -4.51 \\
\hline PRPOP & -4.72 & -4.70 & -4.52 \\
\hline GOPOP & -4.40 & -4.51 & NA \\
\hline POPOP & -4.51 & -4.45 & -4.17 \\
\hline PIPRP (High G) & -3.51 & -3.34 & -3.14 \\
\hline PIPOP (High G) & -3.45 & -3.26 & -3.16 \\
\hline POPRP (High G) & -3.60 & -3.40 & -3.17 \\
\hline PIPIP (High G) & -3.57 & -3.36 & -3.07 \\
\hline PIPRP (Low G) & -5.07 & -4.80 & -4.65 \\
\hline
\end{tabular}




\begin{tabular}{|l|l|l|l|}
\hline PIPOP (Low G) & -4.96 & -4.78 & -4.65 \\
\hline POPRP (Low G) & -4.86 & -4.68 & -4.49 \\
\hline PIPIP (Low G) & -4.61 & -4.61 & -4.49 \\
\hline
\end{tabular}




\section{Conformation Analysis}

The oligomers studied in this work have a twisted conjugated backbone due to the ortho connectivity between the heterocycle and phenylene. Here, we perform conformational analyses using DFT simulation. Our results show that pentamers have two conformations in solution: (1) a Z-shaped conformation, where the molecular backbone has a zig-zag geometry, and (2) a U-shaped conformation, where the molecular backbone has a hairpin shape. Generally, Z-shaped conformation favors the end-to-end conductance pathway (low G) and the U-shaped conformation facilitates an in-backbone conductance pathway (high G).

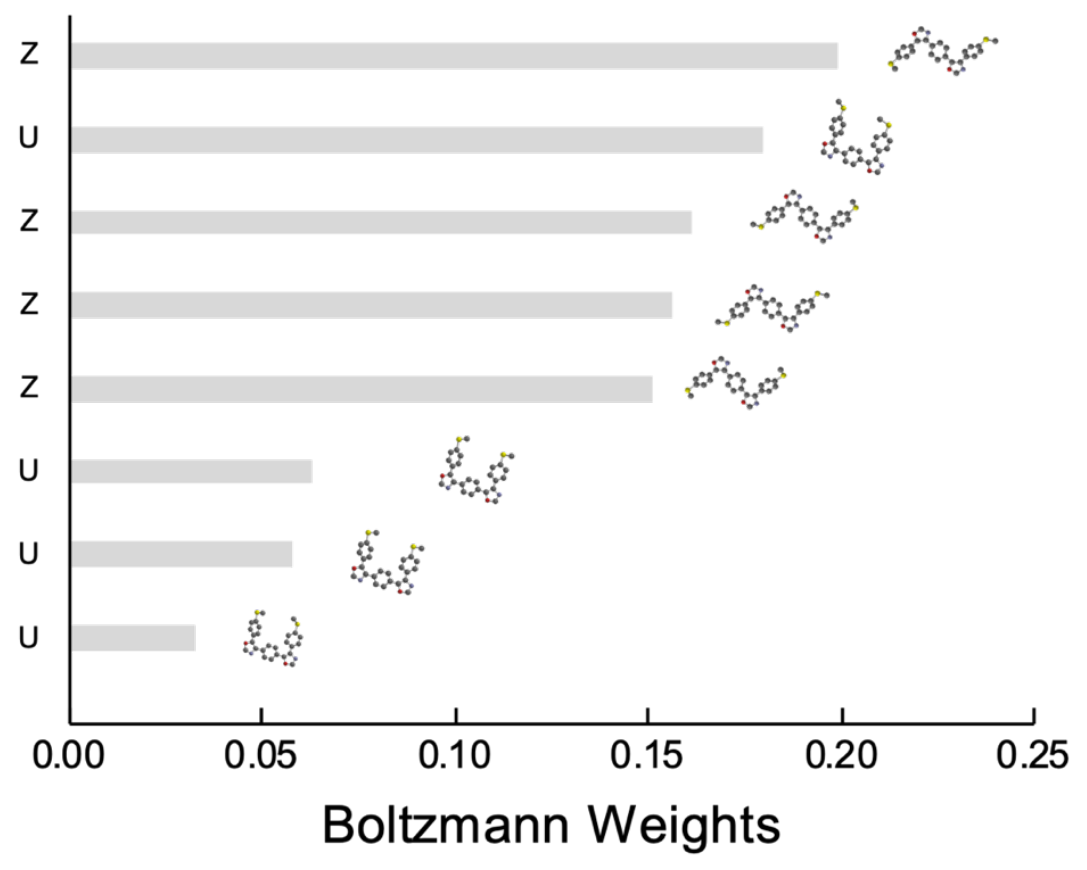

Figure S112. Conformation distribution of (POPOP) based on DFT simulation. Hydrogen atoms are removed for clarity. Conformation ratio of $\mathrm{Z}$-shape/U-shape is 2.0. 


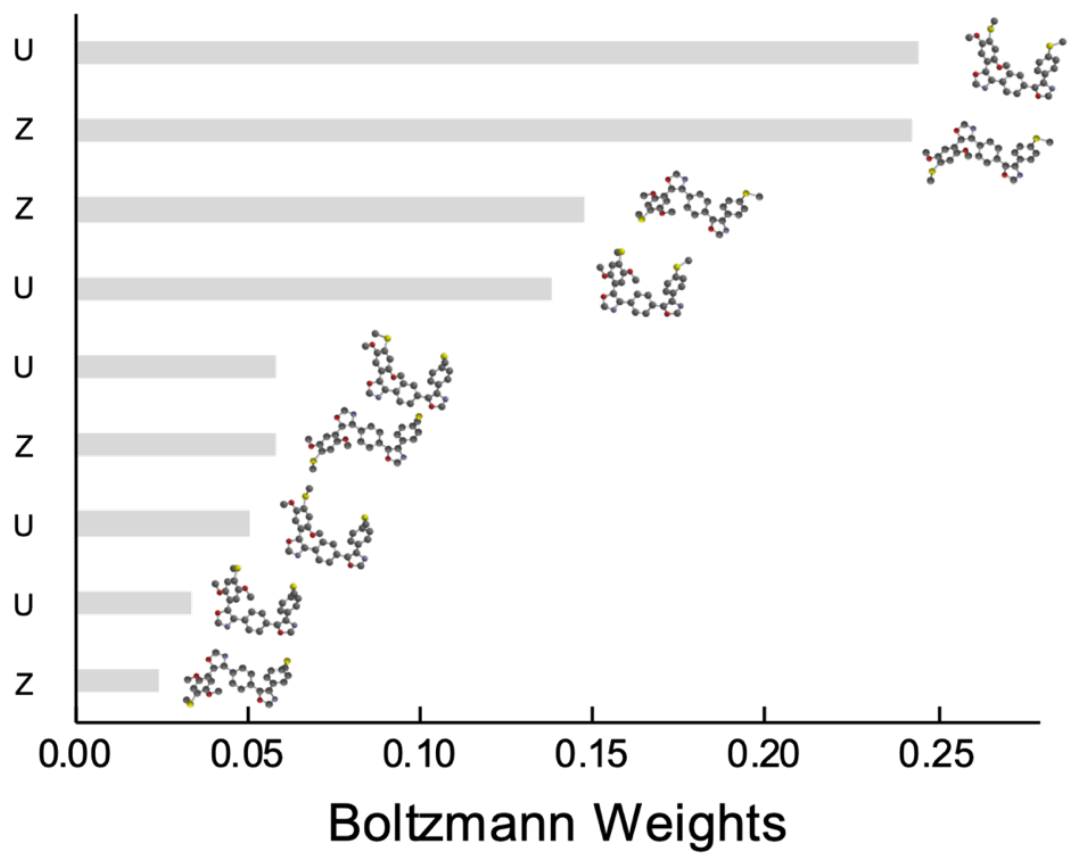

Figure S113. Conformation distribution of (GOPOP) based on DFT simulation. Hydrogen atoms are removed for clarity. Conformation ratio of Z-shape/U-shape is 0.9 .

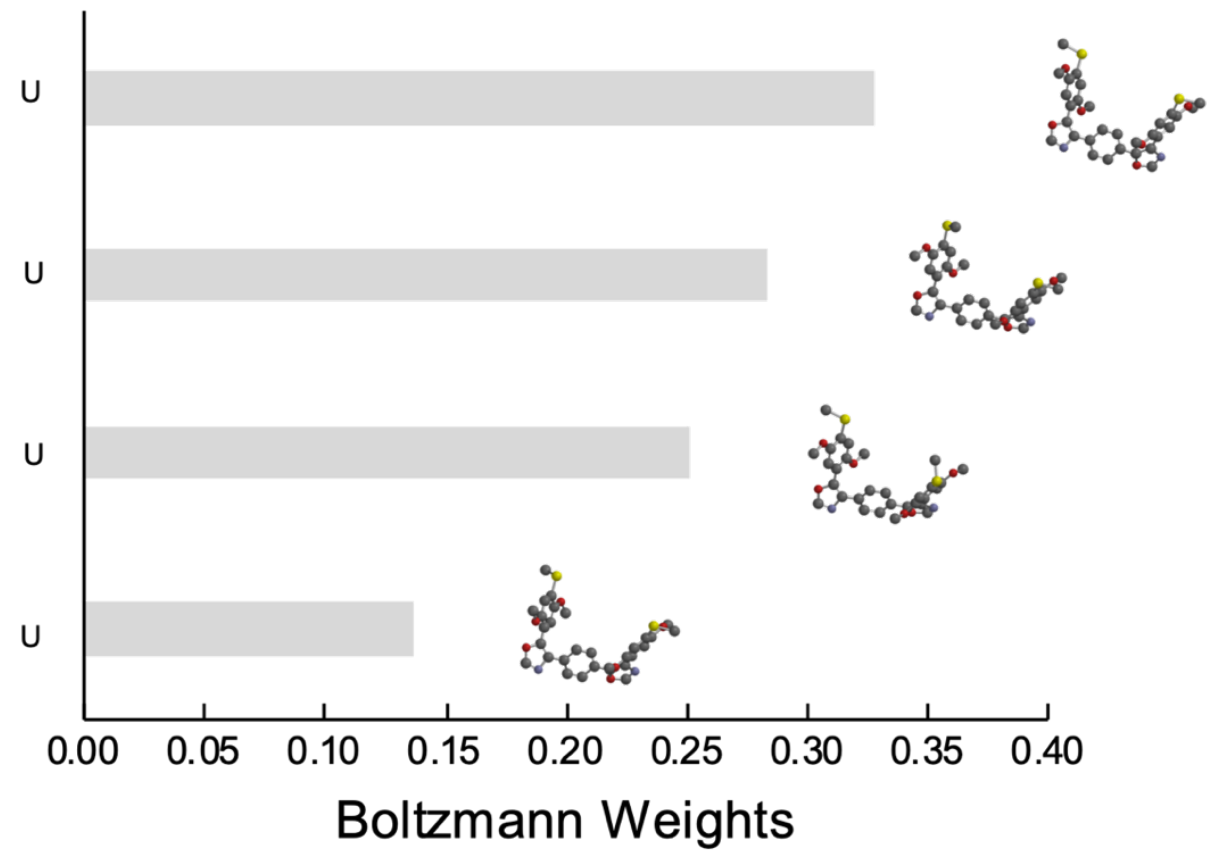

Figure S114. Conformation distribution of (GOPOG) based on DFT simulation. Hydrogen atoms are removed for clarity. Conformation ratio of Z-shape/U-shape is 0 . 


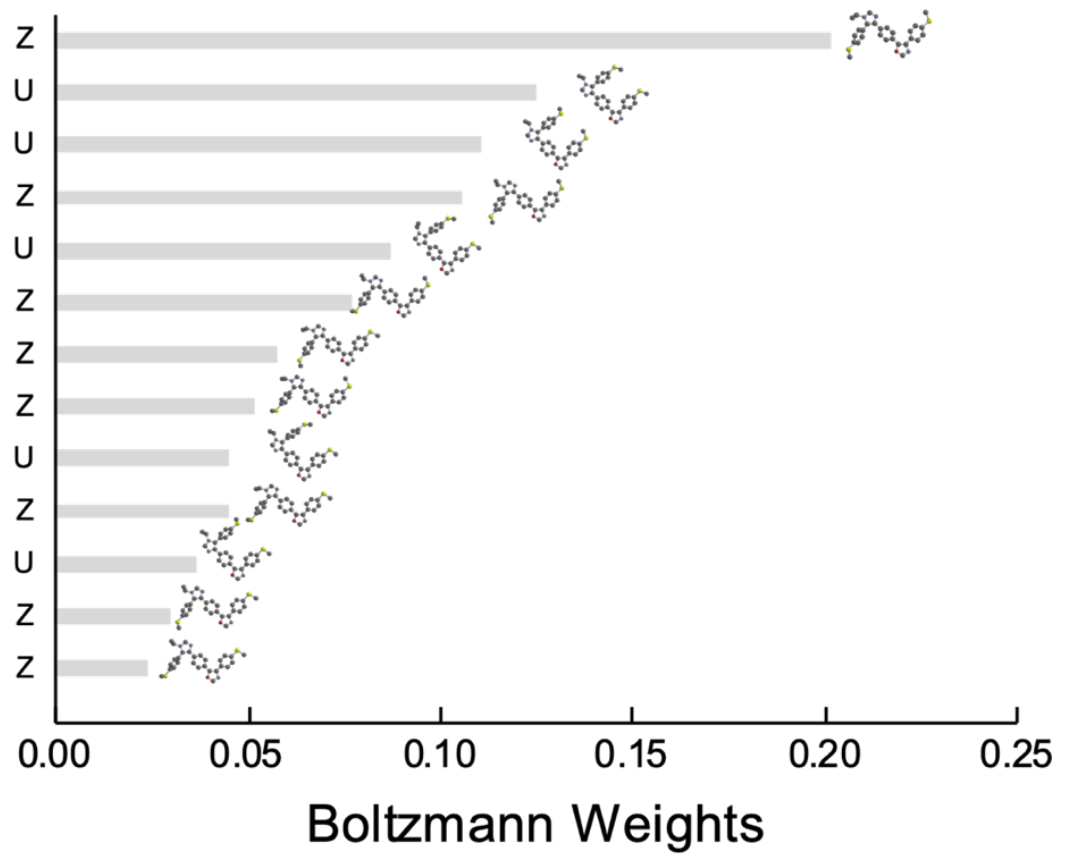

Figure S115. Conformation distribution of (PIPOP) based on DFT simulation. Hydrogen atoms are removed for clarity. Conformation ratio of $\mathrm{Z}$-shape/U-shape is 1.5 .

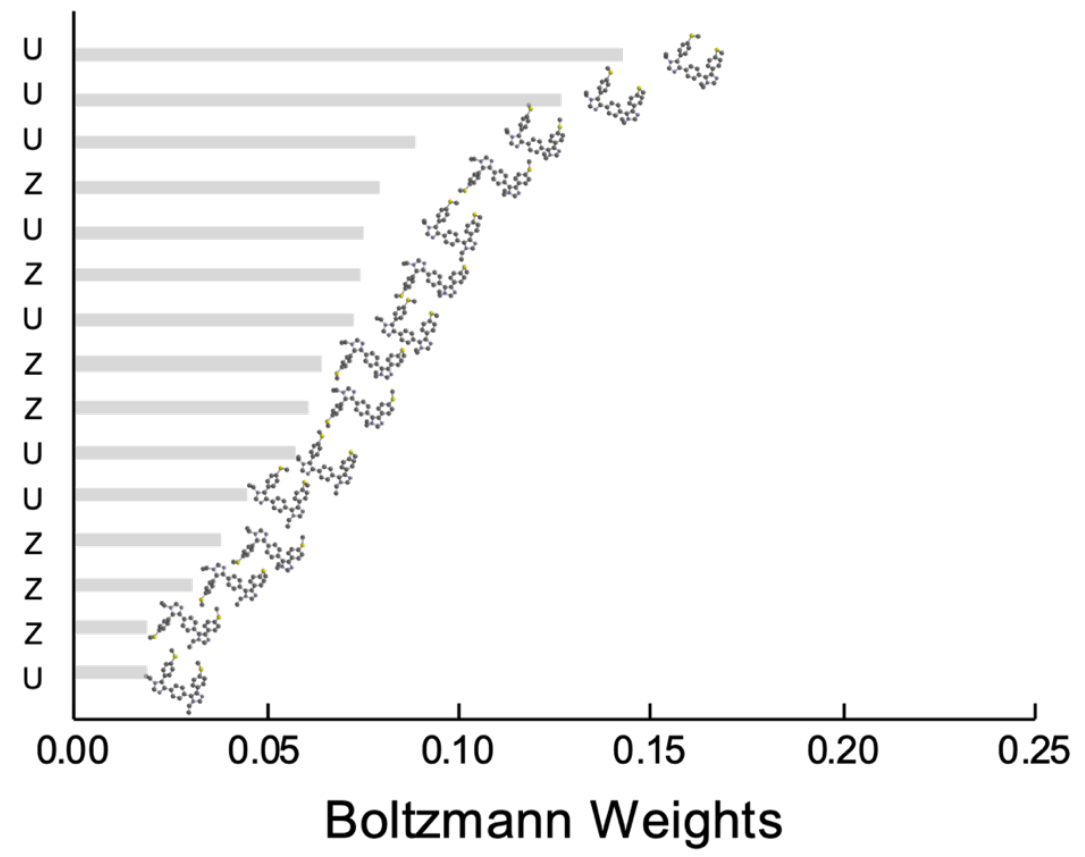

Figure S116. Conformation distribution of (PIPIP) based on DFT simulation. Hydrogen atoms are removed for clarity. Conformation ratio of Z-shape/U-shape is 0.6 . 


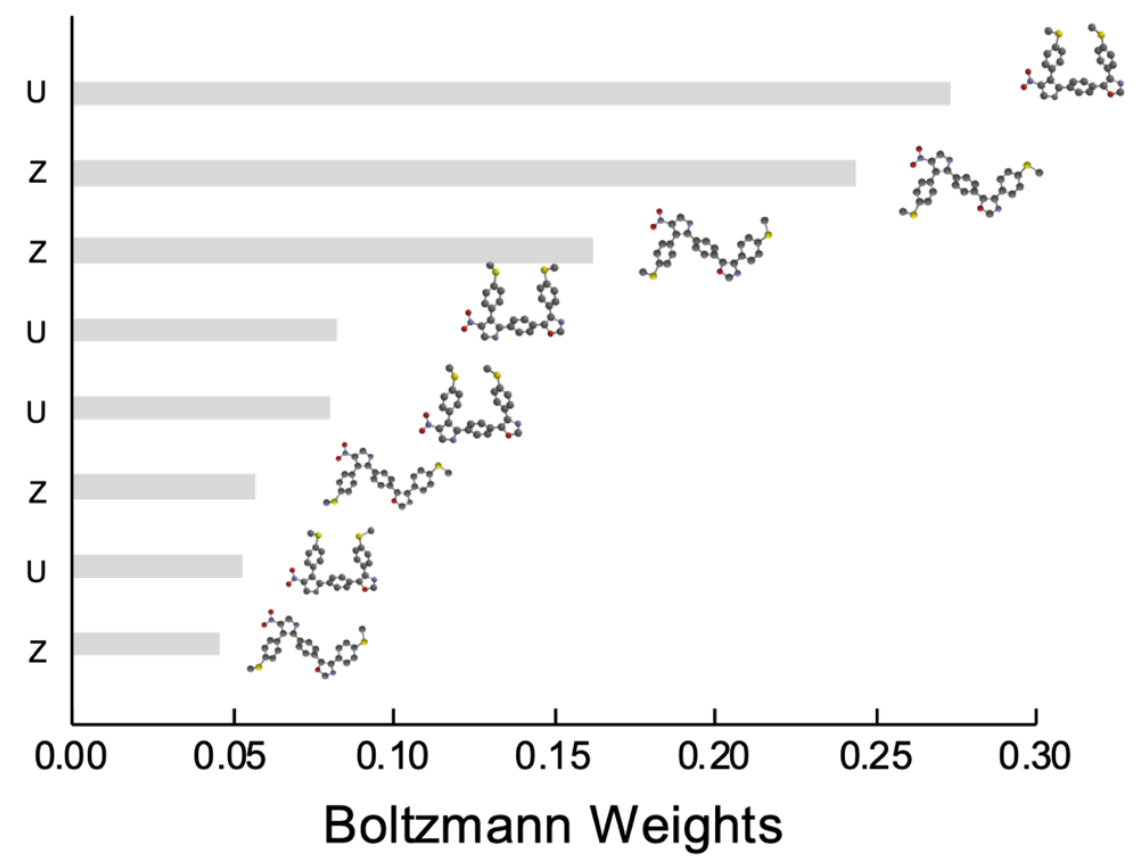

Figure S117.Conformation distribution of (PRPOP) based on DFT simulation. Hydrogen atoms are removed for clarity. Conformation ratio of Z-shape/U-shape is 1.0 .

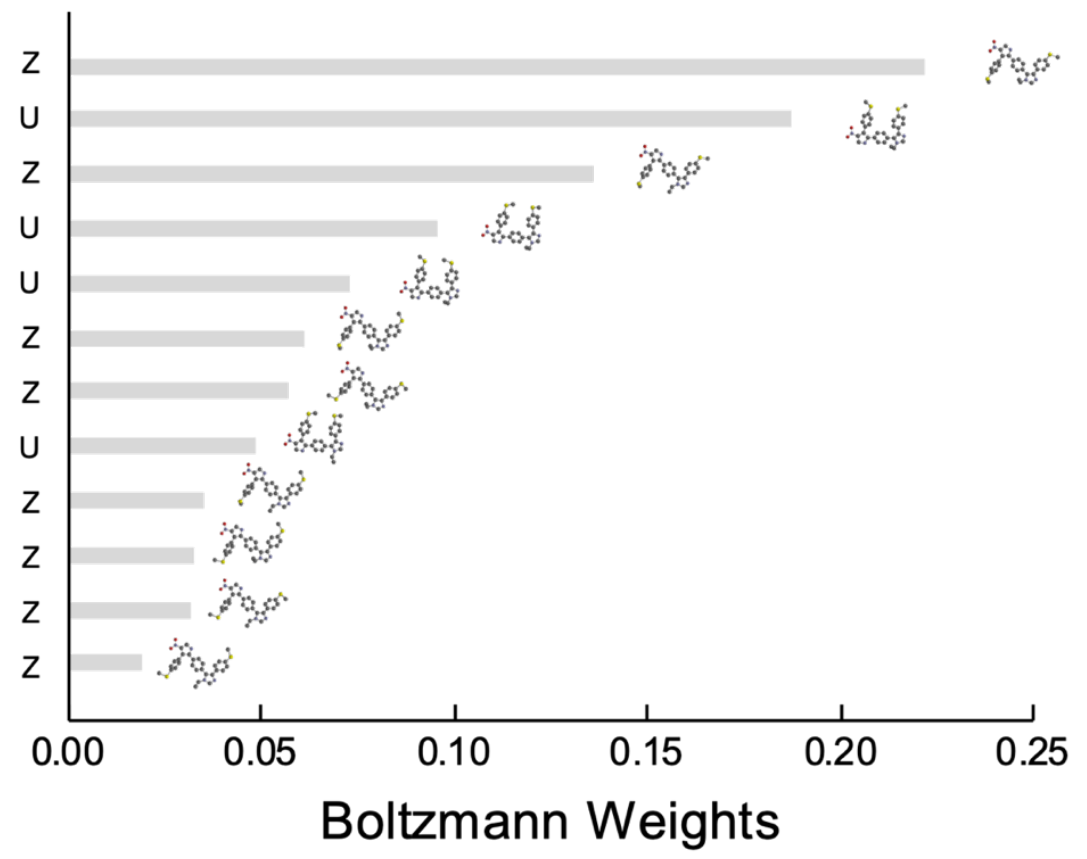

Figure S118. Conformation distribution of (PRPIP) based on DFT simulation. Hydrogen atoms are removed for clarity. Conformation ratio of $\mathrm{Z}$-shape/U-shape is 1.5 . 


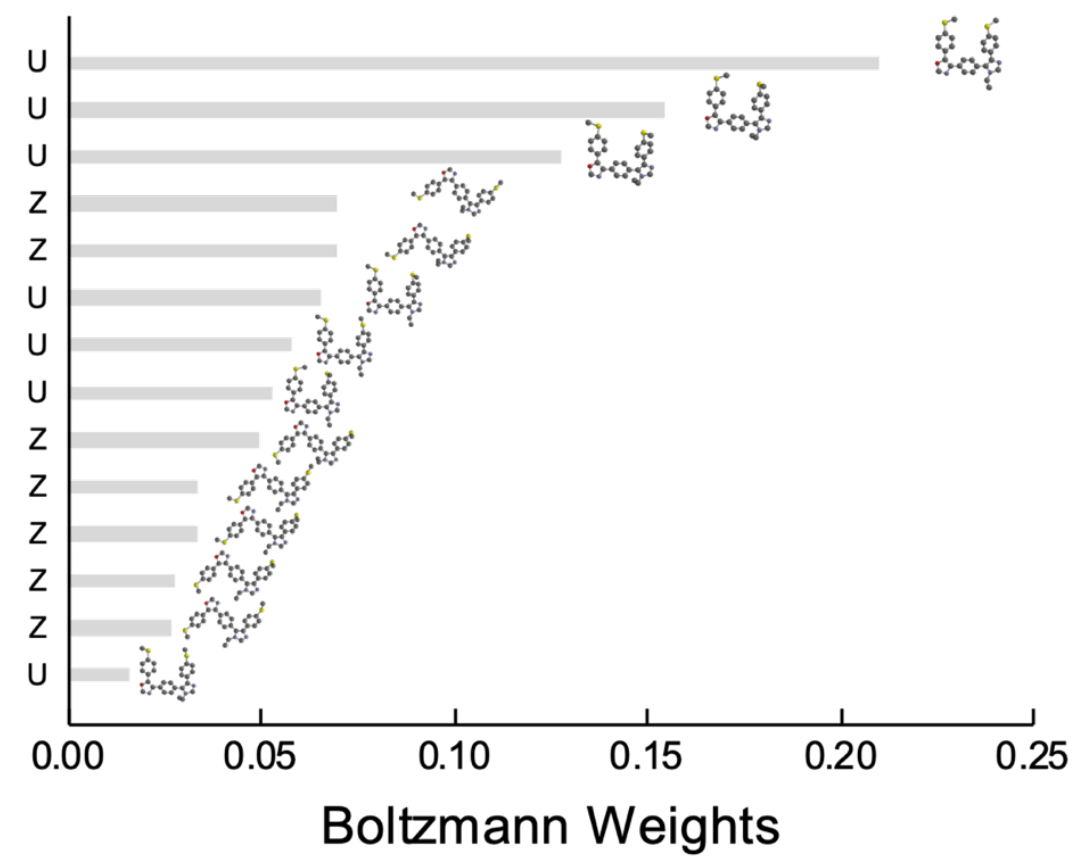

Figure S119. Conformation distribution of (POPIP) based on DFT simulation. Hydrogen atoms are removed for clarity. Conformation ratio of Z-shape/U-shape is 0.5 .

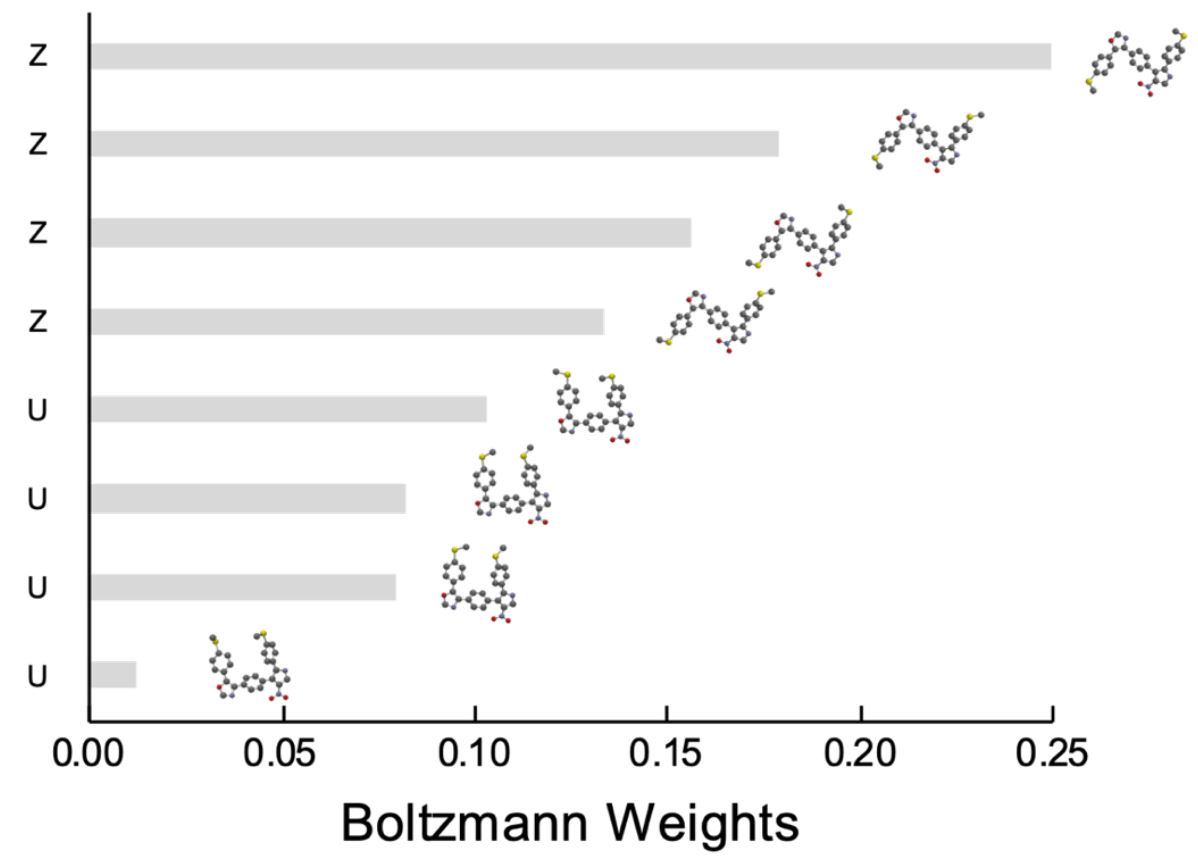

Figure S120. Conformation distribution of (POPRP) based on DFT simulation. Hydrogen atoms are removed for clarity. Conformation ratio of Z-shape/U-shape is 2.6. 


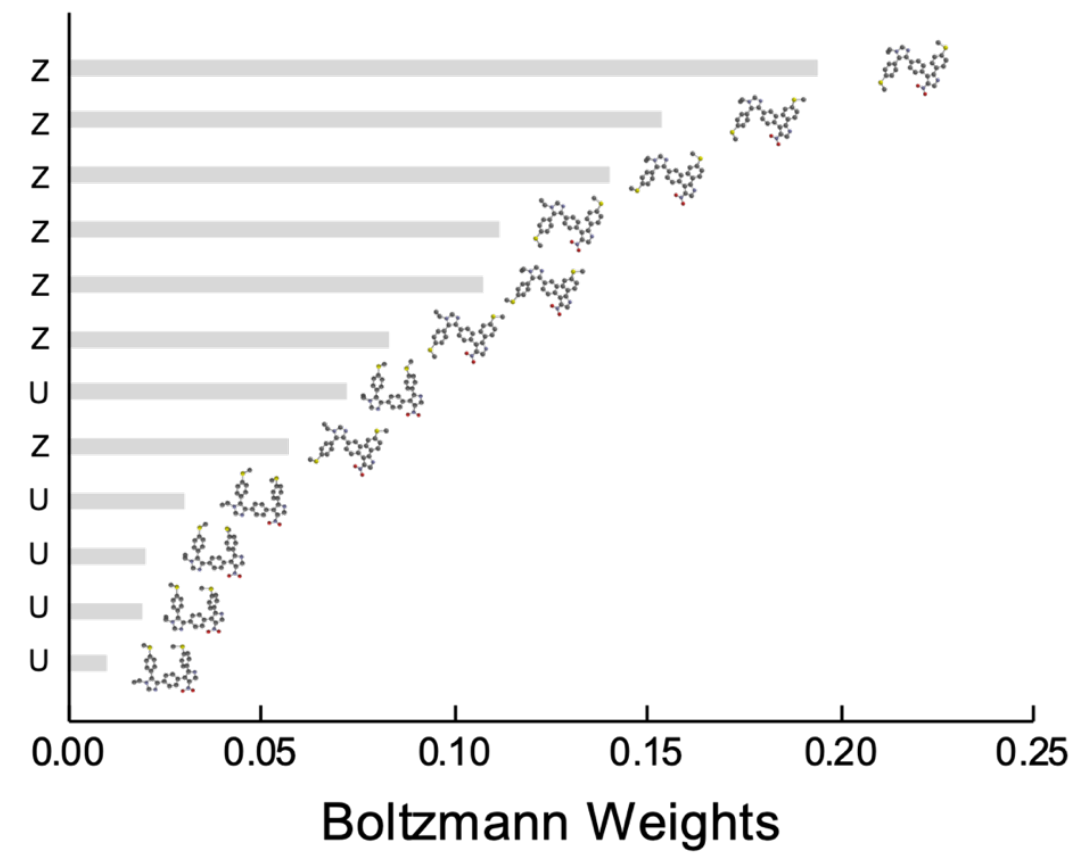

Figure S121. Conformation distribution of (PIPRP) based on DFT simulation. Hydrogen atoms are removed for clarity. Conformation ratio of Z-shape/U-shape is 5.6.

\section{UV-vis Spectra}

Electronic properties are expected to depend on primary sequence in conjugated oligomers ${ }^{9}$. The optical spectra of the heterocycle-phenylene oligomers vary significantly with primary sequence. In general, the absorption maxima are in the range between $275-350 \mathrm{~nm}$.

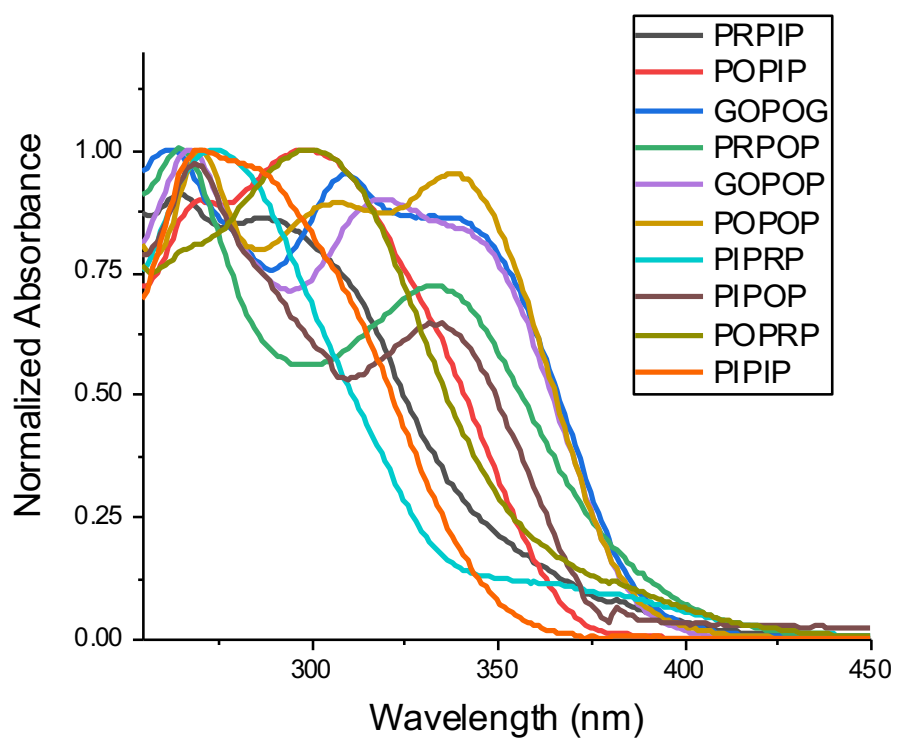

Figure S122. Absorption spectra of pentamers in $\mathrm{CHCl}_{3}$. 


\section{References}

1. $\quad$ Li, B.; Yu, H.; Montoto, E. C.; Liu, Y.; Li, S.; Schwieter, K.; Rodríguez-López, J.; Moore, J. S.; Schroeder, C. M. Intrachain Charge Transport through Conjugated DonorAcceptor Oligomers. ACS Appl. Electron. Mater., 2019, 1 (1), 7-12.

2. Frisch, M. J.; Trucks, G. W.; Schlegel, H. B.; Scuseria, G. E.; Robb, M. A.; Cheeseman, J. R.; Scalmani, G.; Barone, V.; Petersson, G. A.; Nakatsuji, H.; Li, X.; Caricato, M.; Marenich, A. V.; Bloino, J.; Janesko, B. G.; Gomperts, R.; Mennucci, B.; Hratchian, H. P.; Ortiz, J. V.; Izmaylov, A. F.; Sonnenberg, J. L.; Williams; Ding, F.; Lipparini, F.; Egidi, F.; Goings, J.; Peng, B.; Petrone, A.; Henderson, T.; Ranasinghe, D.; Zakrzewski, V. G.; Gao, J.; Rega, N.; Zheng, G.; Liang, W.; Hada, M.; Ehara, M.; Toyota, K.; Fukuda, R.; Hasegawa, J.; Ishida, M.; Nakajima, T.; Honda, Y.; Kitao, O.; Nakai, H.; Vreven, T.; Throssell, K.; Montgomery Jr., J. A.; Peralta, J. E.; Ogliaro, F.; Bearpark, M. J.; Heyd, J. J.; Brothers, E. N.; Kudin, K. N.; Staroverov, V. N.; Keith, T. A.; Kobayashi, R.; Normand, J.; Raghavachari, K.; Rendell, A. P.; Burant, J. C.; Iyengar, S. S.; Tomasi, J.; Cossi, M.; Millam, J. M.; Klene, M.; Adamo, C.; Cammi, R.; Ochterski, J. W.; Martin, R. L.; Morokuma, K.; Farkas, O.; Foresman, J. B.; Fox, D. J. Gaussian 16 Rev. B.01, Wallingford, CT, 2016.

3. Cossi, M.; Rega, N.; Scalmani, G.; Barone, V. Energies, Structures, and Electronic Properties of Molecules in Solution with the C-PCM Solvation Model. J. Comput. Chem. 2003, 24 (6), 669-681.

4. Cai, Z.; Lo, W.-Y.; Zheng, T.; Li, L.; Zhang, N.; Hu, Y.; Yu, L. Exceptional SingleMolecule Transport Properties of Ladder-Type Heteroacene Molecular Wires. J. Am. Chem. Soc. 2016, 138 (33), 10630-10635.

5. Atomistix ToolKit version 2017.12 (QuantumWise A/S, 2017); www.quantumwise.com.

6. Virtual NanoLab version 2017.12 (QuantumWise A/S, 2017); www.quantumwise.com.

7. Van Leusen, A. M.; Wildeman, J.; Oldenziel, O. H. Chemistry of Sulfonylmethyl Isocyanides. 12. Base-Induced Cycloaddition of Sulfonylmethyl Isocyanides to Carbon,Nitrogen Double Bonds. Synthesis of 1,5-Disubstituted and 1,4,5-Trisubstituted Imidazoles from Aldimines and Imidoyl Chlorides. J. Org. Chem. 1977, 42 (7), 1153-1159.

8. Oldenziel, O. H.; Van Leusen, D.; Van Leusen, A. M. Chemistry of Sulfonylmethyl Isocyanides. 13. A General One-Step Synthesis of Nitriles from Ketones Using Tosylmethyl Isocyanide. Introduction of a One-Carbon Unit. J. Org. Chem. 1977, 42 (19), 3114-3118.

9. Zhang, S.; E. Bauer, N.; Y. Kanal, I.; You, W.; R. Hutchison, G.; Y. Meyer, T. Sequence Effects in Donor-Acceptor Oligomeric Semiconductors Comprising Benzothiadiazole and Phenylenevinylene Monomers. Macromolecules, 2016, 50 (1), 151-161. 